Western University Scholarship@Western

Digitized Theses

Digitized Special Collections

1993

\title{
Electrical Strengthening Of Clays By Dielectrophoresis
}

(Julie) Qiulin Shang

Follow this and additional works at: https://ir.lib.uwo.ca/digitizedtheses

\section{Recommended Citation}

Shang, (Julie) Qiulin, "Electrical Strengthening Of Clays By Dielectrophoresis" (1993). Digitized Theses. 2301.

https://ir.lib.uwo.ca/digitizedtheses/2301

This Dissertation is brought to you for free and open access by the Digitized Special Collections at Scholarship@Western. It has been accepted for inclusion in Digitized Theses by an authorized administrator of Scholarship@Western. For more information, please contact tadam@uwo.ca,

wlswadmin@uwo.ca. 


\title{
ELECTRICAL STRENGTHENING OF CLAYS BY DIELECTROPHORESIS
}

\author{
by \\ Julie Qiulin Shang \\ Faculty of Engineering Science
}

Submitted in partial fulfilment

of the requirement for the degree of

Doctor of Philosophy

Faculty of Graduate Studies

The University of Western Ontario

London, Ontario

May 1993

○ Julie Qiulin Shang 1993 
National Library

of Canada

Acquisitions and

Bibliographic Services Branch

395 Wellingion Stre:s

Onawa ontario

KIA ONA
Bibliotheque nationale

du Canada

Direction des acquisitions et

des senvicest llographiques

395. ne Wellington

Otawa (Onlario)

KIA ONA
The author has granted an irrevocable non-exclusive licence allowing the National Library of Canada to reproduce, loan, distribute or sell copies of his/her thesis by any means and in any form or format, making this thesis available to interested persons.
L'auteur a accordé une licence irrévocable et non exclusive permettant a la Bibliotheque nationale du Canaja de reproduire, prêter, distribuer ou vendre des coples de sa thése de quelque manière et sous quelque forme que ce soit pour mettre des exemplaires de cette thèse à la disposition des personnes intéressées.

L'auteur conserve la propriété du droit d'auteur qui protège sa thèse. Ni la thése ni des extraits substantiels de cello-ci ne doivent étre imprimés ou autrement reproduits sans son autorisation. 


\begin{abstract}
Dielectrophoresis, the action of a strong, non-uniform electric field on particles, is potentially a new technique for soil improvement. The principles and technical implementation of dielectrophoretic strengthening of natural clays were investigated in this study. The research included two aspects: the theory of dielectrophoresis in clay-water-electroly.e systems and the experiments of dielectrophoretic treatment on natural clays.

The dielectrophoretic force imposed on a clay-water-electrolyte system is determined by the configuration of the non-uniform electric field and the effective polarizability. The direction of the dielectrophoretic force is governed by the effective polarizability that is in turn controlled by the permittivity and conductivity of the clay-water-electrolyte system.
\end{abstract}

In the experimental program, the effects of dielectrophoresis on two natural clays recovered from Ottawa, Canada were investigated. An electrical potential difference in the order of $15 \mathrm{kV}$ was applied on the clay samples through the insulated electrodes. It was found that the effectiveness of the dielectrophoretic treatment was related to the magnitude and intensity of the electric field intensity. Under different electrical field configurations, the overall soil strengths increased up to $44.0 \%$ after 28 days of treatment. The sensitivity of the clays was reduced up to $70 \%$. 
A comparative study was also conducted to examine the effects of dielectrophoresis and electro-osmosis in the clays. The characteristics of the two methods were discussed.

The dielectrophoretic process is efficient on energy and the electro-chemical reactions associated with the electric current are eliminated. Consequently, the impact to the environment in the process is minimum.

The dielectrophoresis technique may provide potential solutions for the improvement of soft, sensitive clays, consolidation of slurries, remediation of contaminated soils and water, and other applications in geotechnical and environmental engineering based on the findings in this study.

iv 


\section{ACKNOWLEGEMENTS AND DEDICATION}

I wish to express the most sincere gratitude to my chief adviser, Dr. K.Y. Lo and co-adviser Professor I.I. Inculet for their constant guidance, encouragement and support throughout this study, and to Dr. R.M. Quigley for his continuous concern and interest.

The project was supported by the research grant of the National Science and Engineering Research Council of Canada. Jacques-Whitford Lid. provided support for the block sampling project in Orleans, Ontario.

My special thanks are directed to Dr. KT. Law who encouraged me to carry on my doctoral study in the Geotechnical Research Centre at the University of Western Ontario.

I am grateful to Mr. Gary Lusk, Mr. Dave Woytowich, Mr. Wilbert Logan and the: technical staff of the Electronic and Mechanical Workshops of the University of Western Ontario for their assistance during this research.

The generous help from Mr. A. Hefny and Dr. KS. Ho during this study is greatly appreciated.

Finally, I would like to thank my husband, X.J. Li, for his understanding, support and encouragement throughout this study.

This thesis is dedicated to my parents. 
CERTIFICATE OF EXAMINATION

ABSTRACT

ACKNOWLEDGEMENTS AND DEDICATION

TABLE OF CONTENTS

LIST OF TABLES

LIST OF FIGURES

NOMENCLATURE

CHAPTER 1 INTRODUCTION AND OBJECTIVES

CHAPTER 2 LTTERATURE REVIEW

21 Introduction

2.2 Theory of Dielectrophoresis

2.2 Conductivity and

2.23 Permittivity of Matter

2.24 Direction of Dielectrophoretic Force

23 Dielectrophoretic Experiments in Dilute Suspensions

\section{3 .3}

Experiment on a Single

Quartz Sphere

Experiment on Aqueous

Silica

2.3 .4

Summary

24 Dielectrophoretic Experiments in Clays

Work of Lockhart (1983)

24.3 Work of $\mathrm{LO}, \mathrm{Ho}$ and Inculet (1992) 
CHAPTER 3 AN ANALYSIS OF DIELECTROPHORETIC

3.1 Introduction

3.2 Non-Uniform Electric Field

3.2. Composition of Electrode Radial Electric Field in

3.3 Dielectric Phenomena in Aqueous Colloidal Systems

$\begin{array}{lll}3.4 & \text { Effective Polarizability } & 67\end{array}$

$\begin{array}{lll}\text { 3.4.1 Definition } & 67\end{array}$

3.4.2 Factors Influencing Effective

Polarizability

3.4.3

Case 1: Effective Polarizability in a Silica Colloid System

3.4.4 Cave 2: Effective Polarizability in a Kaolinite-Water-Electrolyte 
3.5 Conclusions

CHAPTER 4 GEOTECHMTCAL AND DIELECTRIC PROPERTIES OF TWO SENSITIVE LEDA CLAYS

4.1 Introduction

4.2 Geotechnical Properties 96

4.2.1 Glovester Clay 96

4.22 Orleans Clay 98

$\begin{array}{ll}4.2 .3 & \text { Summary } \\ & 100\end{array}$

$\begin{array}{lll}\text { 4.3 Dielectric Properties } & 101\end{array}$

4.3.1 Experimental Technique 102

4.3.2 Results 106

4.3.3 Summary and Concluaion 110

CHAPTER 5 DIELECTROPHORETIC EXPERIMENTS ON GLOUCESTER CLAY

5.1 Introduction

127

5.2 Apparatus

5.21 Test Chamber

128

5.22 Power Supply

128

5.23

Electrodes

128

$\begin{array}{ll}\text { 5.3 Sample Preparation } & 129\end{array}$

$\begin{array}{lll}5.4 \text { Procedure } & 130\end{array}$

$\begin{array}{lll}5.5 & \text { Results } & 131\end{array}$

5.5.1 Settlement 131

5.5.2 Change of Soil Water Content 131

5.5.3 Change of Soil Shear Strength 133

5.5.4 Summary 134 
5.6 Effect of Intervening Media on Electric

$$
\text { Field Intensity }
$$

5.7 Effect of Polarizability

5.8 Summary and Conclusions

CHAPTER 6 DIELECTROPHORETIC EXPERIMENTS ON ORLEANS CLAY

6.1 Introduction

6.2 Experiment Design and Procedure

6.2.1 Soil Stratigraphy

6.2.2 Test Preparation

152

6.2 .3

Test Procedure

153

6.24

Observations

154

6.2 .5

Post-Treatinent Tests

155

6.26

Summary

156

158

6.3 Results of Tests

6.3.1 Change of Soil Water Content

159

6.3.2

Settlement

162

6.3.3

Change of Undrained Shear

Strength

162

6.3.4

6.3 .5

Stress-Strain Behaviours

164

6.3.6

Sensitivity

164

6.3.7

CIU Triaxial Tests

165

6.3 .8

Consolidation Tests

166

Atterberg Limits Tests

166

6.3 .9

Chemical Analynes

167

6.4 Further Discussion
6.4 .1
Effects of Electric Field
6.4 .2
Intensity and Gradient
6.4 .3
Dissociation of Soil Water
Inter-particle Forces 
CHAPTER 7 ELECTRO-OSMOSIS IN ORLEANS CLAY:

7.1 Introduction

7.2 An Overview on Electro-Osmosis and Dielectrophoresis

7.3 Review of Theory

7.4 Test Set-Up

7.5 Procedure and Observation

7.6 Results

7.6.1

Settlement

7.6.2

Negative Pore Water Pressure

206

7.6 .3

Soil Water Content

207

7.6 .4

Shear Strength

7.6.5

Stress-Strain Curve, Undrained

Modulus and Sensitivity

7.6.6

Preconsolidation Pressure

211

7.6 .7

Physical and Chemical Changes

7.7 Further Comparison of Electro-Osmosis and Dielectrophoresis

7.8 Conclusions

CHAPTER 8

SUMMARY, CONCLUSIONS AND RECOMMENDATION FOR FURTHER RESEARCH

8.1 Summary and Conclusions

8.2 Recommendation for Further Research

\section{APPENDIX 1 DISTRIBUTION OF CYLINDRICAL} EIECTRIC FIELD 
PAGE

APPENDIX 2 EXAMPLE CALCULATION OF A

KAOLINITE-WATER-ELECTROLYTE SYSTEM

251

APPENDIX 3 ELIMINATION OF ELECTRODE

POLARIZATION

254

APPENDIX $4 \quad$ X-RAY TRACES

257

VITA

274 


\section{LIST OF TABLLS}

Table

Description

Page

21 Conductivity and Relative Permittivity of

Various Materials

2.2 Test Results for Wallaceburg Clay Samples

23 Test Results for Gloucester Clay Samples

24 Summary of Dielectrophoretic Test

Results for Gloucester Clay Samples

3.1 Dielectric Properties of

Kaolinite-Water-Electrolyte System

3.2 Stem Potential and Permittivity

Increment of Commun Clay Minerals

in Water-Electrolyte

4.1 Summary of Soil Propertics

4.2 Summary of Chemical Analysis

4.3 Summary of Composition Data

4.4 Dielectric Properties of Leda Clays

5.1 Summary of Experiment Results on

Gloucester Clay

6.1 Changes of Soil Water Content

after Treatment

6.2 Summaries of Unconfined Compression

Test Results

6.3 Summary of Consolidation Test Results

6.4 Summary of Atterberg Limits Test Results

6.5 Summary of Chemical Analysis Results 
7.2 Physical and Chemical Properties before and after Treatment 


\section{LIST OF FIGURES}

Figure

Description

Page

21

An Electric Dipole in an External Electric

Field

23 DEP Test Cell for a Single Quartz Sphere

24 Dielectrophoresis on a Single Quartz Sphere

25 DEP Test Cell for Aqueous Colloid Silica

26 DEP Force Vs. Frequency in Aqueous Colloid Silica

High-Voltage Electrode

3.1 Perfect Cylindrical Electric Field Geometry

3.2 Equi-Intensity Lines and Electric Field

Lines in a Cylindrical Field

3.3 Electrode-Pair

3.4 Equi-Intensity Lines of a Pair of Cylindrical Electrodes

3.6 Equi-Intensity Lines of an Electrode-Matrix

3.7 Electrical Double Layer: Gouy-Stem Model

$3.8 \quad$ Illustration of Double Layer Polarization 
3.11 Effective Polarizability of Clay-water-electrolyte Systems.

3.12 Silica Particle in Water

4.1 Geotechnical Profile of Subsoil Condition in Gloucester Test Fill Site before

Treatment

4.2 X-Ray Diffraction Traces of Gloucester Clay

4.3 Borehole Record at Orleans Site

4.4 Block Sampling: Overview of Excavation

4.5 A Close View to Block Sampling

4.6 X-Ray Diffraction Traces of Orleans Clay

4.7 Test Cell

4.8 Teat Set-Up: Measurement of Soil Electrical Properties

4.9 Test Cell for Measurement of Soil Electrical Properties

4.11 Conductivity of Two Leda Clays

4.12 Structure of Sensitive Clay

$5.1 \quad$ Teat Apparatus

5.2 Soil Sample Preparation

5.3 Profile of Gloucester Clay at Test Location

5.4 Test Set-Up: Gloucester Clay Tube Samples

5.5 Typical Soil Settiement Va. Treatment Time 
Non-Uniform Electric Field with Cylindrical Symmetry

$5.8 \quad$ Distribution of Electric Field

Intensity along Radial Direction

6.1 Properties of Orleans Clay

$6.2 \quad$ Typical Shear Stress Vs. Vertical

Strain in Unconfined Co:npression Tests

6.3 Test Set-up for Orleans Clay

6.4 Photo of Test Set-up: Orkans Clay Block Sample

6.5 Electrode Configurations

6.6 Block Sample after Treatment, Test No. B2

6.7 Crack Pattern: After Test No.B3

6.8 Experimental Design: Post-Treatment Soil Testing

6.9 Contour Map Showing Post-treatment

Soil Water Content, Test No.B2

6.10 Contour Map Showing Post-treatment

Soil Water Content, Test No.B3

6.11 Contour Map Showing Post-treatment

Soil Water Content, Teat No.B4

6.12 Soil Settlement During Treatment

6.13 Contour Map Showing Soil Shear

Strength after Test B4

6.14 Typical Shear Stress Vs. Strain in Unconfined Compresuion Tests before and after Treatment for Orleans Clay 
6.15 CIU Triaxial Test Results before and after Treatment

6.16 Variation of Strength Envelope before and after Treatment

6.17 Results of Consolidation Tests before and after Treatment

6.18 Increase of Shear Strength in Relation to Gradient of $E^{2}$

Increase of Soil Shear Strength in

Relation to Electric Field Intensity

Soil Sensitivity Vs. Carbonate Content

6.21 Interparticle Force between Particles

7.1 Crose-Section of Capillary 221

$7.2 \quad$ Electro-Osmotic Cell

7.3 Electric Current Polarity during Treatment

7.5 Settlement and Pore Water Pressure at Anode with Time, Test GV-8

7.6 Pore Pressure after 24-Hour Treatment

7.8 Changes of Soil Water Content and Shear Strength after Treatment

7.9 Moisture Content before and after Treatment, Test GV-8 
Figure

Description

7.10 Shear Strength before and after Treatment

Test GV-8

7.11 Results of Unconfined Compression Tests

7.12 Consolidation Test Results before and after Treatment

7.13 Consolidation Curves for Gloucester

Clay Samples before and after Treatment

233

A3.1 Eqquivalent Circuit of Electrode

Polarization 
NOMENCLATURE

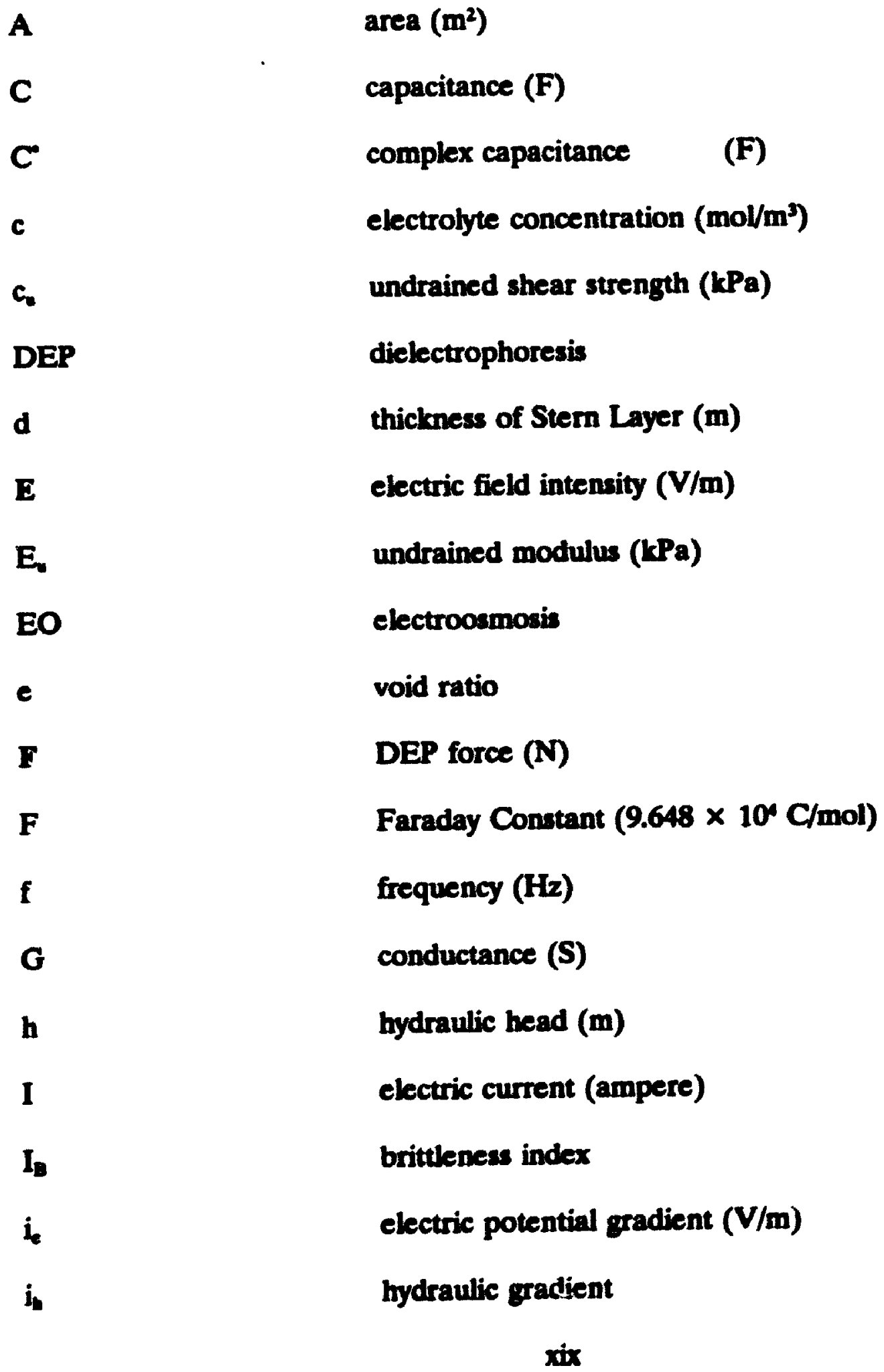




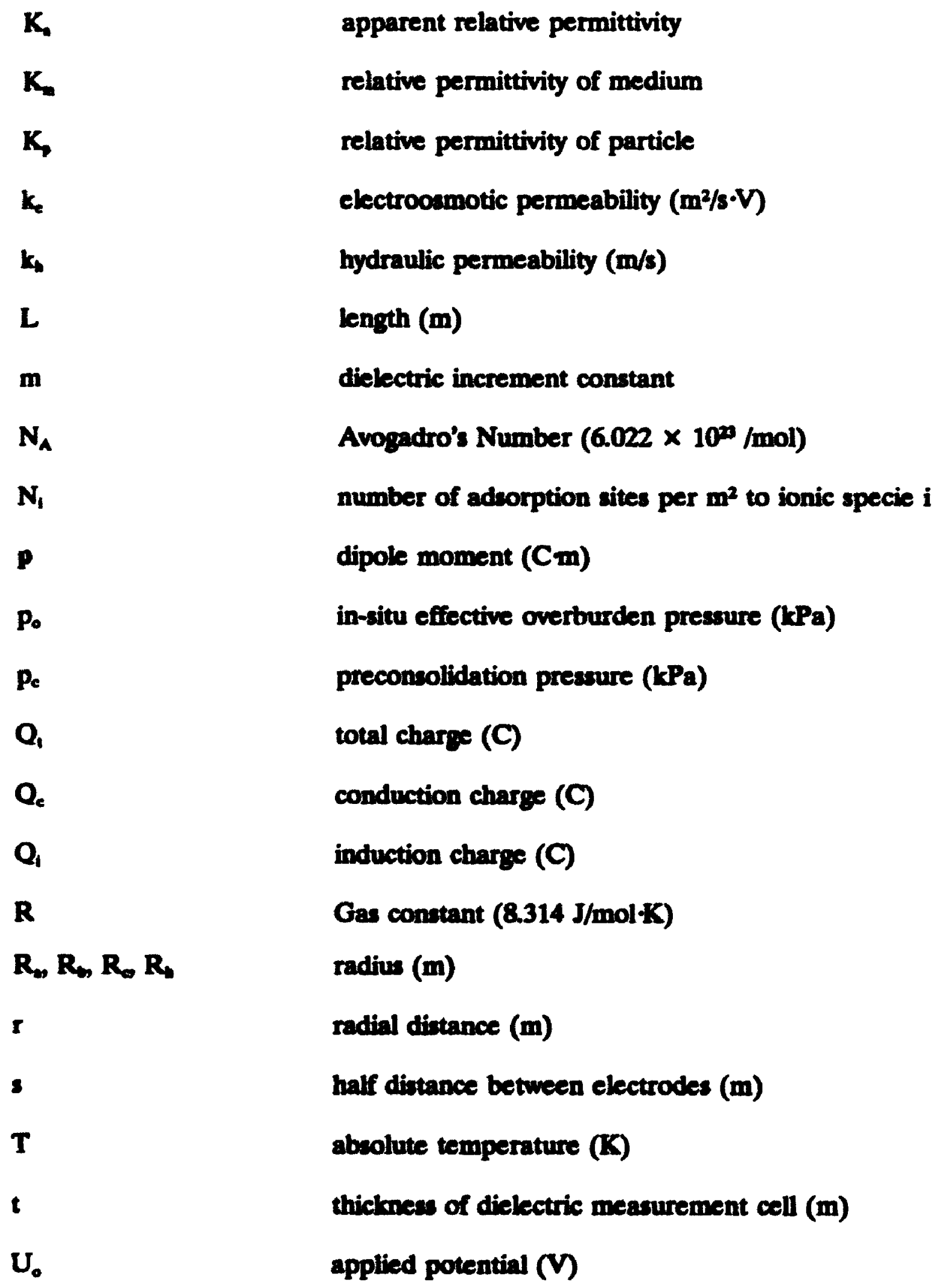


$\boldsymbol{\alpha}$

$\alpha_{\text {ext }}$

$\boldsymbol{\alpha}_{\mathbf{x}}$

$a_{\text {xced }}$

$\gamma$

$\boldsymbol{\epsilon}$

$\epsilon^{\circ}$

$\epsilon^{\prime \prime}$

$\epsilon_{\circ}$

$\epsilon$.

$\epsilon_{\boldsymbol{m}}$

$\epsilon_{\mathrm{p}}$

$\boldsymbol{\xi}$

$\boldsymbol{\kappa}$

$\lambda$

v

$\rho, \rho_{1}, \rho_{2}$

$\boldsymbol{\sigma}$ pore water pressure $(\mathbf{k P a})$

voltage (V)

volume $\left(\mathbf{m}^{3}\right)$

water content (\%)

valence

polarizability (F/m)

effective polarizability (F/m)

relative polarizability

relative effective polarizability

unit weight $\left(\mathrm{kN} / \mathrm{m}^{3}\right)$

permittivity (F/m)

complex permittivity (F/m)

out-of-phase permittivity ( $F / m)$

permittivity of vacuum, $8.854 \times 10^{-12} \mathrm{~F} / \mathrm{m}$

apparent permittivity (F/m)

permittivity of medium (F/m)

permittivity of particle (F/m)

zeta potential (V)

Constant of Gouy diffuse layer $\left(\mathrm{m}^{-1}\right)$

electric field configuration constant

density (kg/m?

charge denaity $\left(\mathrm{C}_{\mathrm{m}} \mathrm{m}^{2}\right)$

conductivity (S/m)

woi 


\begin{tabular}{|c|c|}
\hline$\sigma_{-}$ & conductivity of medium (S/m) \\
\hline o, & conductivity of particle (S/m) \\
\hline T & relaxation time (second) \\
\hline$\tau_{\text {eal }}$ & effective relaxation time (second) \\
\hline$\Phi$ & surface potential (V) \\
\hline $\boldsymbol{\Phi}$. & Stem potential (V) \\
\hline$\Phi$ & zeta potential (V) \\
\hline$\phi$ & specific adsorption potential (J) \\
\hline $\boldsymbol{\omega}$ & angular frequency $(\mathbf{H z})$ \\
\hline
\end{tabular}


The author of this thesis has granted The University of Western Ontario a non-exclusive license to reproduce and distribute copies of this thesis to users of Western Libraries. Copyright remains with the author.

Electronic theses and dissertations available in The University of Western Ontario's institutional repository (Scholarship@Western) are solely for the purpose of private study and research. They may not be copied or reproduced, except as permitted by copyright laws, without written authority of the copyright owner. Any commercial use or publication is strictly prohibited.

The original copyright license attesting to these terms and signed by the author of this thesis may be found in the original print version of the thesis, held by Western Libraries.

The thesis approval page signed by the examining committee may also be found in the original print version of the thesis held in Western Libraries.

Please contact Western Libraries for further information:

E-mail: libadmin@uwo.ca

Telephone: (519) 661-2111 Ext. 84796

Web site: http://www.lib.uwo.ca/ 


\section{CHAPTER 1}

\section{INTRODUCTION AND OBJECTIVES}

Dielectrophoresis is the action of non-uniform electric fields on suspended particles caused by polarization effects. The concept of dielectrophoresis was first introduced by Professor H.A.Pohl in 1951. Since then dielectrophoresis has grown rapidly in use for mineral, colloidal, and cellular biological studies. In his book titled Dielectrophoresis (1978), Pohl summarized the mechanism and applications of the technique. Among scientists working on the topic. T.B. Jones (1979) made important contribution to the theoretical aspect regarding the dielectrophoretic force calculation. The dielectrophoresis in clay-water-electrolyte systems was investigated for the first time by Lockhart in 1983. The incentive to apply principle of dielectrophoresis in geotechnical engineering was credited to Inculet and Lo (1988) who illustrated convincingly through an experiment that dielectrophoresis could become a powerful tool to improve the engineering properties of soft clays. Lo et al (1992) further investigated the effects of dielectrophoresis on strengthening sofi clays and the factors influencing the process in an experimental program.

This thesis is a continuation of the research on dielectrophoresis in clay-waterelectrolyte systems. To apply the technique of dielectrophoresis in geotechnical engineering is the ultimate goal of the research. The direct objective of the research is to explore the theoretical and experimental aspects of dielectrophoretic process in natural clay soils. The objective may be further introduced in two categories: 
(A) To develop the theory of dielectrophoresis in clay-water-electrolyte systems. This includes the dielectrophoretic force, the non-uniform electric field, the electric behaviour of clay-water-electrolyte systems, the effective polarizability, and the physico-cheinical effects. Furthermore, a comparative study is targeted to distinguish the characteristics of dielectrophoresis and electroosmosis in clays.

(B) To examine the theoretical aspects by experimental investigations. The Leda clays recovered from Ottawa, Canada were used throughout the study. The experimental investigation was aimed on the following topics:

(1) Geotechinical and electric properties of the Leda clays;

(2) The effects of intervening media to the dielectrophoresis treatment;

(3) The changes of soil properties after dielectrophoresis treatment;

(4) The effects of physico-chemical reactions;

(5) Electro-osmosis experiments in the Orieans clay to compare dielctrophoresis and electroosmosis in terms of strengthening effects.

The theoretical and experimental investigation conducted in this study will establish the fundamental principles of dielectrophoresis in clays. It is hoped that the technique will provide solutions in some geotechnical engineering problems, including improvement and stabilization of soft, sensitive clays, control of foundation settlement and environment related aspects. 


\section{CHAPTER 2}

\section{LITERATURE REVIEW}

\subsection{Introduction}

The action of a non-uniform electric field on particles has been investigated since 1940's. As far back as in the work of Winslow (1949), it was found that silica gel in kerosine in a strong, non-uniform electric field is structurized, forming a fibrous, ropy mass. Similar observations were made by McEuen (1966), who noted the formation of fibrous structures in clay suspensions at the site of maximum field intensity, which he termed "bridges". Pohl (1951) was the first to adopt the term "dielectrophoresis", initially defined as the motion of neutral particles in a nonuniform electric field. For the case in which dielectrophoresis leads to coagulation, Pohl (1978) has introduced the term "mutual dielectrophoresis". It has been further noted by Stoylov and Pekanchin (1975), Shilov and Estrela-L'Opis (1975) and Pohl (1978) that charged particles in an electrolytic solution would also acquire motion in a non-uniform alternating electric field, which Stoylov and Pekanchin termed "dipolophoresis". Since charged particles acquire mobility in a uniform electric field as well (electrophoresis), Shilov and Estrela-L'Opis (1975) suggested that the term "dipolophoresis" should be taken to mean the component of mobility of a charged particle that is caused by the non-uniform ac field, in which the particle displacement induced by electrophoresis, on the average of the period, is zero. Therefore, the sole effect of dielectrophoresis can only be fully illustrated in an alternating electric field.

In this study, the term "dielectrophoresis" ("DEP" in abbreviation) is used 
throughout to represent the action of a non-uniform ac or dc electric field on particles which may be either neutral or charged. The study was carried out with alternating current electric fields ( ac fields) exclusively. Dielectrophoresis in ac fields requires considerably less energy loss in the medium.

The principles of dielectrophoresis are introduced in this chapter based on the research conducted by numerous scientists, followed by a review of experimental works with respect to investigations on the mechanism and practical applications.

\subsection{Theory of Dielectrophoresis}

\subsubsection{Dielectrophoretic Force}

The theory of dielectrophoresis was explained primarily in terms of the dielectrophoretic force (Jones, 1979): A small dielectric particle can be treated as a simplified dipole consisting of two equal and opposite electric charge (Q) with a vector distance (d) apart in an external electric field, Fig.2.1 (a) and (b). When the external field is non-uniform, it affects one charge of the dipole more than the other, so the dipole acquires motion in the direction of greater field intensity. The simplest derivation of the dielectrophoretic force is accomplished by summing the forces on the charges $( \pm Q)$ in a non-uniform electric field, $E$, as shown in Fig.2.1(c). The net force on this dipole is:

$$
y=g z(z+d)-g z(z)
$$

where $r$ is the space coordinate vector. If $d \rightarrow 0$ and only the lowest-order term in $d$ is retained: 


$$
\boldsymbol{P}-Q(d \cdot \nabla) \mathbf{E}
$$

The product $(Q d)$ is interpreted as an electric dipole moment $p$, so we have

$$
F=(p \cdot \nabla) \Sigma
$$

Eq.(2.3) is the generally accepted expression of dielectrophoretic force, where $p$ is the dipole moment vector of the particle, $\nabla$ is the del vector and $E$ is the external electric field intensity.

By using the vector transformation

$$
(A \cdot \nabla) B=\nabla(A \cdot B)-(B \cdot \nabla) A-A \times(\nabla \times B)-B \times(\nabla \times A)
$$

Then Eq.(2.3) may be written as

$$
\boldsymbol{F}=\nabla(\boldsymbol{p} \cdot \boldsymbol{E})-(\boldsymbol{E} \cdot \nabla) p-p \times(\nabla \times \boldsymbol{E})-\boldsymbol{\Sigma} \times(\nabla \times p)
$$

For steady or static electric field,

$$
\boldsymbol{\nabla} \times \boldsymbol{I}=0
$$

and for cases where the material consisting the particle is homogeneous and isotropic,

$$
\boldsymbol{p}=\boldsymbol{\alpha} \boldsymbol{v} \boldsymbol{z}
$$


where $a$ is the polarizability constant and $v$ is the volume of the particle, respectively. Substitute Eqs.(2.6) and (2.7) into (2.5) and re-organize, we have

$$
\boldsymbol{F}=\frac{1}{2} \propto V \nabla E^{2}
$$

Eq.(2.8) is the basic equation of dielectrophoretic force imposed on a single particle. It may be noted that the dielectrophoretic force depends directly upon the first power of the volume and polarizability of the particle, but upon the square of the electric field intensity. The latter result emphasizes that dielectrophoresis is independent of the sign of the field direction. It can occur in an ac field.

It should be emphasized that the dielectrophoretic force in Eq.(2.8) is based on several assumptions, all of which are (Jones, 1979):

(1). The particle must be small compared to the scale of the external non-uniform field;

(2). The electric field intensity must not be appreciably affected by the particle;

(3). The electrostatic approximation must be valid;

(4). The dielectric properties of the particle must be homogeneous, linear and isotropic.

\subsection{Conductivity and Permittivity of Matter}

The interaction of matter with an electric field is described by its dielectric behaviours, which occur in either of two ways: as a conduction or as a polarization (Hill et al, 1969). Charges which are free to move through the sample show up as 
a conduction term. If their motion is blocked. their response appears as a polarization.

Periodic processes are described most simply by the complex representation of physical properties. For example, a sinusoidally varying voltage

$$
V(t)=V_{0} \cos \omega t
$$

may be replaced by its complex analog

$$
V(t)=V_{s} e^{j \omega m}
$$

The concept of complex permittivity is introduced in order to describe polarization phenomena in real matter having finite permittivity and conductivity. Consider a parallel plate capacitor filled with a material with permittivity $\epsilon$ and conductivity $\sigma$. Thus the total measured charge on the capacitor plates is equal to the sum of the conduction and induction charges, i.e.

$$
Q_{c}=Q_{c}+Q_{i}
$$

where $Q_{t}$ is the total charge, $Q_{e}$ is the conduction charge and $Q_{i}$ is the induction charge.

The conduction charge and induction charge can be expressed in the complex plane as 


$$
\begin{aligned}
& Q_{c}=\int I d t=\frac{I}{i \omega}=-\frac{V G}{i \omega} \\
& Q_{1}=V C
\end{aligned}
$$

where $\mathrm{i}=\sqrt{ }-1$,

$$
\begin{aligned}
& \text { I : electric current in ampere, } \\
& w \text { : angular frequency in hert, } \\
& \text { V : voltage on the capacitor in volt, } \\
& \text { C : capacitance in farad and } \\
& \text { G : conductance in siemens (1/ohm ). }
\end{aligned}
$$

Substitute (2.10) into (29), the total charge may be expressed in the form

$$
Q_{t}=V C+\frac{V G}{i \omega}=V C^{*}
$$

in which

$$
C^{*}=C+\frac{G}{i \omega}=\left(c-i \frac{0}{\omega}\right) \frac{A}{d}
$$

here

$C^{*}$ : complex capacitance;

E : permittivity, unit: farad/m;

$\sigma$ : conductivity, unit: siemens/m;

A : plate area of capacitor plates; unit: $\mathbf{m}^{2}$;

d : separation distance of capacitor; unit: $\mathbf{m}$.

We may then introduce a complex permittivity from Eq.(2.12) 


$$
e^{*}=e-i e^{\prime \prime}
$$

with the conversion relations

$$
e^{\prime \prime}=\frac{0}{\omega}
$$

where

$\epsilon^{*}:$ complex permittivity;

$\epsilon:$ in-phase permittivity ( static permittivity);

$\epsilon^{\text {": }}$ out-of-phase permittivity;

$\sigma$ : conductivity;

$\omega$ : angular frequency.

As expressed in the complex plane, the two quantities, conduction and polarization, appear in the electric equations as the in-phase and out-of-phase permittivities, which may be lumped together as a "complex permittivity", $\epsilon^{\circ}$.

In practice, the relative permittivity (dielectric constant), $K$, is used more often. The relative permittivity is defined as

$$
K=\frac{e}{e_{0}}
$$

where $\epsilon_{0}=8.85 \times 10^{-12}(\mathrm{farad} / \mathrm{m})$, is the permittivity of vacuum.

In a summary, the dielectric properties of any real matter which has finite nonzero permittivity and finite non-zero conductivity can always be described by 
Eq.(213). Conductivity and permittivity are measures of conduction and polarization of matter, respectively. " $\sigma$ " is a larger quantity for easily conductive materials and a smaller quantity for insulators and semiconductors. Similarly, with exception of metals, " $\epsilon$ " is a larger quantity for easily polarized materials and a smaller quantity for other materials. Table 2.1 lists the conductivities and dielectric constants of several materials. It may be seen from Table 2.1 that at dry state, most minerals, soils and rocks can be classified as dielectric materials with low conductivities and the relative permittivities ranged between 3 to 17. Metals are good conductors with conductivity ranged in the order of $10^{6}$ to $10^{7} \mathrm{~S} / \mathrm{m}$ and permittivity approaching to infinity. Plexiglas and Transformer oil are good insulation materials which are used in this study. Water is considered as one of the most difficult material in terms of dielectric properties. In general, the relative permittivity of water is in the order of 80 and indifferent of small amount of electrolytes. On the other hand, the conductivity of water varies substantially with electrolyte concentrations. The pure, dejonized water has the conductivity in the order of $10^{-9} \mathrm{~S} / \mathrm{m}$, which can be considered as an insulator, whereas the sea water may have the conductivity of 4 $\mathbf{S} / \mathbf{m}$, which is practically a conductor.

\subsubsection{Polarizability}

The polarizability is a parameter describing the behaviour of a disperse system consisting of many dispersed particles suspended in a continuous medium. The examples of disperse systems include air bubbles in transformer oil, clay particles suspended in water-electrolyte, and $s 0$ on. Consider a finite dielectric particle of 
volume $\mathbf{v}$ in a fluid medium. The dielectric properties of the particle and medium may be described by the complex permittivity defined in Eq.(2.13). For the simple situation of an uncharged particle, following the work of Pohl (1978), Jones (1979) and Benguigui and Lin (1982), the polarizability $\sigma$ is given by

$$
a=3 \operatorname{Re}\left(\epsilon_{m} \frac{e_{p}^{*}-e_{m}^{*}}{e_{p^{0}}+2 \epsilon_{m}^{*}}\right) \quad(F / m)
$$

where

$R e=$ real part of the complex conjugate;

Subscript $\mathbf{p}=$ particle;

Subscript $\mathbf{m}=$ medium.

The other notations are as defined before.

Introducing a relaxation time $r$,

$$
\tau=\frac{e_{p}+2 e_{m}}{\sigma_{p}+2 \sigma_{p}} \quad(\text { second })
$$

and solving the real part of Eq.(2.16),

$$
\alpha=3 \epsilon_{m}\left(\frac{\omega^{2} \tau^{2}}{1+\omega^{2} \tau^{2}} \frac{\epsilon_{p}-\epsilon_{p}}{\epsilon_{p}+2 \epsilon_{p}}+\frac{1}{1+\omega^{2} \tau^{2}} \frac{\sigma_{p}-\sigma_{m}}{\sigma_{p}+2 \sigma_{m}}\right)
$$

where $\sigma$ is the polarizability of the system.

Similar to introduction of relative permittivity, a relative polarizability, $\sigma_{\mathrm{K}}$, may be defined as 


$$
\alpha_{R}=\frac{a}{e_{0}}
$$

The relative polarizability $\sigma_{\mathrm{x}}$ is sometimes more convenient for practical use.

Eq.(2.18) is the general equation of polarizability of suspension systems. For cases in which the dielectric properties of particle and medium remain constant over the frequency range of interest, the relaxation time $r$ is a constant which represents the intrinsic dielectric behaviour of the system.

It is instructive to note that dielectrophoresis effect has built-in restrictions as regards the roles of polarizability. Eq.(2.18) shows that the sign and magnitude of polarizability depend on the differences of the dielectric properties of particle and medium. The polarizability and consequently, the dielectrophoretic force on the particle will be zero if the particle and medium have identical conductivity and permittivity.

Depending on the relaxation time of a particle-medium system in an external field with constant frequency, the polarizability may be categorized in three characteristic regimes:

(1) Dielectric regime.

Suppose that the particle and medium have zero-conductivity, i.e.,

$$
\theta t>1
$$

then the system is said in the dielectric regime, in which the polarizability defined in Eq.(2.18) approaches the limit as $\omega r \rightarrow \infty$ : 


$$
\alpha=3 e_{-} \frac{c_{p}-\varepsilon_{e}}{\varepsilon_{p}+2 \varepsilon_{m}}
$$

Eq.(2.20) is the original expression suggested by Pohl (1978) for "ideal dielectrics", i.e., both particle and medium have zero conductivities and zero dielectric loss. An example of the typical systems located in the dielectric regime is the air bubbles suspended in transformer oil (Jones and Kallio, 1979).

(2) Conductive regime.

Many materials may not be treated as ideal dielectrics, i.e., they have finite non-zero conductivities. Clay-water-electrolyte systems consisting of clay mineral particles and water-electrolytes are the typical examples. A system is considered in a conductive regime at the limit of

$$
\omega \tau<<1
$$

as $\omega r \rightarrow 0$, the polarizability approaches to

$$
\alpha=3 e_{\omega} \frac{\sigma_{p}-\sigma_{p}}{\sigma_{p}+2 \sigma_{p}}
$$

Eq.(2.22) shows that in the conductive regime, the polarizability is dominated by the conductivities of the particle and medium as well as the permittivity of the medium, whereas not dependent on the permittivity of the particle.

(3) Transient regime.

Systems under the condition between the two extremes may be categorized 
in transient regime when

$\omega \tau \approx 1$

As $\omega r \rightarrow 1$, the polarizability of the system is then

$$
\alpha=\frac{3}{2} \epsilon_{m}\left(\frac{e_{p}-e_{m}}{\epsilon_{p}+2 \epsilon_{m}}+\frac{\sigma_{p}-\sigma_{m}}{\sigma_{p}+2 \sigma_{m}}\right)
$$

2.2.4. Direction of Dielectrophoretic Force

Eq.(2.8) reveals that dielectrophoretic force vector $F$ on a small particle depends on three basic quantities: the volume of the particle, the gradient of square of electric field intensity and the polarizability. The first quantity is always positive; the sign of the second quantity will remain unchanged once the electric field and space coordinates are set up. The direction of dielectrophoretic force, therefore, is governed solely by the last quantity, i.e., the polarizability. In other words, the tendency of motion of particles in a non-uniform electric field may be towards either the higher or the lower fields, depending on the sign of polarizability. At a constant frequency of external electric field, the sign of polarizability may be reversed by changing the dielectric properties of the particle and medium.

A convention is followed in this study thereafter with respect to the direction of dielectrophoresis effects:

(1). Positive DEP. The sign of polarizability is positive. The dielectrophoretic force is towards the direction of higher field intensity. Particles in the field tend to 
move into the high-intensity region;

(2). Negative DEP. The sign of polarizability is negative. The dielectrophoretic force is towards the direction of lower field intensity. Particles in the field tend to move away from the high-intensity region.

\subsection{Dielectrophoretic Experiments in Dilute Suspensions}

This section presents a review of dielectrophoresis in dilute suspensions in order to verify the validity and limitation of the theoretical aspects discussed in the Section 2.2. As discussed before, the major concerns with regard to the action of dielectrophoresis are the magnitude and direction of the dielectrophoretic force. The experiments reviewed in this section may help to understand the mechanism involved and to discover the limitation of the theory. The first experiment can be considered as an "ideal" case to demonstrate the action of dielectrophoresis. The second experiment presents a single quartz sphere hanging in an aqueous solution where the action of dielectrophoretic force was observed through deflection of the sphere. The third experiment involved an aqueous silica colloid system where difficulty of the theory arises. We shall see that the basic theory is no longer valid in tt. ; third case. Thus further exploration to the mechanism is required.

\subsection{An "Ideal" DEP Experiment}

Pohl and Schwar (1959) conducted a series of tests to investigate the behaviour of suspended particles in a poorly conductive dielectric liquid. The suspension consisted of PVC (poly-vinyl chloride) particles sized 90 to $213 \mu \mathrm{m}$ 
suspended in a liquid having a closely similar density ( $\mathrm{CCl}_{4}$ and benzene mixture). Both materials are weakly conducting with conductivities in the order of $10^{-12} \mathrm{~S} / \mathrm{m}$. Hence the system is located within the dielectric regime, as discussed in Section 2.2. The particles and supporting liquid have relative permittivities of 4.58 and 2.26 , respectively. The frequency of applied ac field was $60 \mathrm{~Hz}$. We may calculate the polarizability $a$ of the system from Eq.(2.18) as $+15.3 \times 10^{-12} \mathrm{~F} / \mathrm{m}$. The corresponding relative polarizability $\sigma_{1}$ is +1.73 from Eq.(2.18a). It is noted that the calculation according to the dielectric regime approach based on Eq.(2.20) gives the same value of polarizability up to the third-decimal place, confirming that the system is located in the dielectric regime. The dielectrophoretic force is expected to be positive, i.e., the particles should be attracted to the central electrode, where the field is more intense.

The test cell and its electrodes had a cylindrical geometry, as shown in Fig.22(a). The central electrode, $0.8 \mathrm{~mm}$ in diameter with Teflon coating of $0.13 \mathrm{~mm}$ in thickness, was mounted in the centre of the test cell. The outer electrode was the grounded test cell, having the inside diameter of $9.8 \mathrm{~mm}$. The movement of PVC particles were observed during tests. The particles could be collected at the central electrode as they moved into the region.

- Polarizability. In the test series the particles moved to the central electrode and were collected there, indicating positive dielectrophoresis, as predicted by the theory. The percentage of particle collection (yield in percentage) is plotted against the applied voltage in Fig.22(b). The test duration for all tests were 180 seconds.

- Enect of particle size. The larger particles were pulled to the central electrode 
more quickly than the smaller ones, as shown in Fig.2.2(b). This is also predicted by the theory, Eq.(2.8), in which the dielectrophoretic force is proportional to the particle volume, $v$.

- Enect of electric neld intensity. In Fig.2.2(b), the Yield-versus-Voltage curves have parabolic shape at low voltage (about $2 \mathrm{kV}$ ). However, as the voltage increases, the curves turn into a rather linear fashion with a flat slope. Above a critical voltage, no collection occurs. It is almost as if the field were entirely cut off. Pohl (1978) has attributed the critical "cut-off" voltage to the "trajectory effect" due to the conductive behaviours of the system. He has shown that the critical voltage is inversely related to the particle size, but proportional to the central electrode diameter and is affected by the degree of insulation of the central electrode. The mechanism of the critical "cut-off" voltage is still under investigation. Up to date, it can be determined only from experimental data. For practical purposes, we should carefully choose the applied voltage below the "cut-off" value in application of dielectrophoresis. Furthermore it should be noted that the yield of particles remained nearly unchanged in a rather large range as the voltage approached the critical value. Therefore it is desirable to utilize the parabolic portion in the Yieldversus-Voltage curve for the best result.

In general, the experiment conducted in a weakly conducting system presented good agreement with the theory in certain scope. The direction of dielectrophoretic force, the effects of particle size and electric field strength are verified. However, the existence of critical "cut-off" voitage and the "flat" pattern on the yield curve before the critical voltage revealed the complexity of the mechanism. 


\subsubsection{Experinien: on A Single Quartz Sphere}

The response of particles suspended in aqueous media to a non-uniform electric field has been investigated since 1960 's. Problems arise due to the fact that substances that do not ionize in water are usually found to be negatively charged when in contact with water, and the addition of small amounts of electrolyte tends to increase this charge (Bockris and Reddy, 1970). The questiun was first addressed by Chen (1967). The effect of dielectrophoresis on a single quartz sphere in water-electrolytes solutions was observed. As shown in Fig.2.3, a solid quartz sphere approximately $1 \mathrm{~mm}$ in diameter was hung on a fine glass fibre within a test cell. The electric field had cylindrical geometry. The aqueous solutions with various concentrations and conductivities were introduced into the cell to surround the hanging sphere. The deflection of the sphere under the effect of non-uniform field was monitored with the aid of a microscope. A positive deflection denotes motion toward the region of higher field intensity (positive DEP) and a negative deflection denotes motion away (negative DEP).

The quartz and water have the relative permittivity of 3.78 and 80 , respectively. Negative dielectrophoresis will be expected in the system under various conductivities of the aqueous solutions, according to Eq.(2.18). The experiment showed that the prediction was true. Negative dielectrophoresis was observed in all tests, as shown in Fig.2.4(a), where the relationship between the deflection (DEP force) and resistivity of the solutions are plotted. Note that by definition, resistivity (ohm $\mathrm{cm}$ in unit) is the reciprocal of conductivity. It may be seen that the magnitude of deflection (DEP force) varied with the resistivity of solutions and was affected 
slightly by the valences of cations. We can calculate the relative polarizability $\alpha_{k}$ at various conductivities based on Eq.(218) and Eq.(2.18a) and plot on the same figure (Fig.2.4(b)). It is appreciated that the theory provides fairly good description to the trend of negative dielectrophoretic force imposed on the quartz sphere. As seen in Fig.2.4(b) that the polarizability of the quartz-water-electrolyte systems calculated according to the conductivity and permittivity of the quartz particle and aqueous media is always negative regardless the conductivity of the media. The negative sign in the prediction is attributed to the fact that aqueous media almost always have higher permittivity and conductivity than those of silica particles, Eq.(218). It is noted that the magnitude of polarizability is much larger than the PVC-CCl,-benzene system discussed in Section 2.3.1 as the permittivity of water is much higher than those of $\mathrm{CCl}_{4}$-benzene mixtures, indicating that dielectrophoresis could be more effective in aqueous systems.

As shown in the experiments, the behaviour of the quartz sphere in a nonuniform field is generally in agreement with the theory in spite of some effects due to existence of cations. It can be concluded that the effect is largely controlled by the volumetric effects rather than the surface effects because of the relatively large particle size.

\subsubsection{Experiment on Aqueous Silica}

The effect of dielectrophoresis on colloid sized silica gel in aqueous suspensions was investigated by Scrimager (1974). The silica gel $\left(\mathrm{SiO}_{2}\right)$ used in Scrimager's series had the analogous dielectric permittivity and conductivity as those 
of quartz used in Chen's series. The major factor distinguished Scrimager's test series from those of Chen's was the particle sizes. In Scrimager's tests, the silica particles were sized in the colloid range of $0.2 \sim 0.5 \mu \mathrm{m}$, whereas in Chen's test, a single quartz sphere of $1 \mathrm{~mm}$ in diameter was used.

The silica particles were prepared by passing commercial (DuPont Ludox) colloidal silica through an ion exchange resin to deionize it. The material after drying and grinding was sized by sedimentation to the desired particle-size range, washed with deionized water several times, then re-suspended in chosen solutions of known pH and composition.

The test cell was designed to allow the colloid suspension flowing through the cell where a non-uniform electric field was applied, as shown in Fig.2.5. The particles could be collected at either high or low field intensity region, depending on the polarizability of the system. The dielectrophoretic collection was observed with a microscope having a calibrated reticle.

Contradictory to Chen's results, the dielectrophoretic force was positive in Scrimager's tests, i.e. the particles were attracted to the region of highest field intensity and collected there. The phenomenon suggests that the system demonstrate a practically positive polarizability to the outsider observer, against the prediction according to the theory.

The experiment was conducted under various conditions with unanimous results in terms of the direction of particle motion. The author presented the results in the yield-versus-frequency curves. The typical data are presented in Fig.2.6. The positive DEP was presented as the positive DCR (dielectrophoretic collection rate) 
in the figures. Two distinct collection peaks can be observed in each curve. The collection rate varies with $\mathrm{pH}$ of the solution and particle size. The author found that the particle concentration and cations in the solution would also affect the collection rate. Pohl interpreted the feature of frequency dependence of the collection rate as the contribution of relaxation time, defined in Eq.(217). As the interest of this study is dielectrophoresis in geotechnical applications, we shall focus the discussion in the low frequency $(\mathrm{f}<100 \mathrm{~Hz}$ ) range. Detailed discussion with respect to the frequency dependence of dielectrophoresis can be found in Pohl's book Dielectrophoresis (1978).

In spite that the magnitude of dielectrophoretic force was affected by many factors, the direction of the force is not affected, as evidenced by particle motion. Further observation indicates that the force is little affected by these factors at low frequencies, as we may see in Fig.2.6.

The similar observation has been reported on many investigations involving aqueous suspensions (Shilov and Rozen, 1975; Pohl, 1978; Santamaria et al, 1985; Inoue et al, 1988; etc). The behaviours of aqueous colloid suspensions at the frequency ranged below the order of $1 \mathrm{MHz}$ are universally anomalous. These experimental findings may not be interpreted by the simple expression of polarizability in Eq.(2.18) and we should think of further advances to the theory of dielectrophoresis. Details of interpretation of this anomaly are given in Chapter 3.

\subsubsection{Summary}

The cases reviewed in this section indicate that

(1). The theory of dielectrophoresis may well describe the behaviour of suspension 
systems consisted of weakly conducting materials in a non-uniform electric field, with exception of the critical "cut-off" voltage, which can be determined experimentally. (2). The dielectrophoretic experiments in aqueous solutions have demonstrated diverging effects:

- The behaviour of a solid dielectric sphere of relative large in size hanging in an aqueous solution is generally in agreement with the theory. The negative dielectrophoresis was observed in the system;

- The positive dielectrophoresis was discovered in the colloid sized silica particles suspended in an aqueous solution, which can not be interpreted by the original theory presented in the Section 2 of this chapter.

The experiments reviewed in this section show that the dielectric behaviours of aqueous colloid systems have their unique characteristics. In order to apply the principles of dielectrophoresis to the geotechnical engineering practice, these behaviours must be understood.

\section{Dielectrophoretic Experiments in Clays}

Over the past thirty years dielectrophoresis has grown rapidly to the point where it is currently in use for mineral, colloidal and cellular biological studies. However these applications are primarily in dilute suspensions. The effort to apply dielectrophoresis in dewatering, consolidating and strengthening clays in natural status started in 1980's. Lockhart (1983) conducted an experimental series on synthetic kaolinite and montmorillonite to study dielectrophoresis in drained clay suspensions. Although some indications of water migration was observed, no significant results 
was obtained due to technical reasons. The effort to apply the principle of dielectrophoresis in geotechnical engineering for strengthening natural soft clays is credited to Inculet and Lo (1988). They conducted a group of pioneer tests showing that dielectrophoresis could significantly improve the strength properties of natural clays. Further experimental exploration on dielectrophoretic treatment of soft clays was recently reported by Lo, Ho and Inculet (1992), in which further evidence of the effectiveness of dielectrophoresis on soil improvement was provided.

In this section, these experimental sturlies on dielectrophoresis in clays are reviewed.

\subsection{Work of Lockhart (1983)}

Lockhart carried out a series of laboratory tests on synthetic kaolinite and montmorillonite as part of his research on dewatering mining tailings.

The kaolinite of sodium, hydrogen and aluminium were prepared by treating $10 \%$ suspensions of the kaolinite with approximately $0.33 \mathrm{M}$ solutions of $\mathrm{NaCl}, \mathrm{HCl}$ and $\mathrm{Al}_{2}\left(\mathrm{SO}_{4}\right)_{3}$ respectively. After two such treatments, the suspensions were washed five or six times with conductive water, centrifuging and re-dispersing each time to remove excess electrolyte. The montmorillonites of sodium, hydrogen and aluminium were prepared in similar method.

The test cell comprised coaxial non-insulated stainless steel electrodes, $2.0 \mathrm{~cm}$ and $7.5 \mathrm{~cm}$ in diameter, mounted inside a plexiglas cylinder $10 \mathrm{~cm}$ in diameter. The electrodes were fabricated from perforated sheets and surfaces which faced each other were covered with filter paper. The samples were placed in the 
annular space between the two electrodes. The plexiglas base had small holes at the centre and at the rim from which water percolating through the inner and outer electrodes, respectively, could be collected. An ac electric field of frequency $50 \mathrm{~Hz}$ was applied by a power supply with 280 volt capacity.

The flow rate of water from the samples which were still draining by gravity was registered. It was found that the flow rate at the central electrode was higher than those under the effect of gravity only, indicating that the water migrated towards the highest field intensity. As evidenced, the clay particles were attracted away from the highest field, i.e. the negative dielectrophoresis has taken place.

No water flow was recorded for the completely drained samples $(\sim 30 \mathrm{Wt} . \%$ solids, estimated water content $~ 55 \%$ ), suggesting that the magnitude of the dielectrophoretic force was small. It is also observed that the samples became overheated even at relatively low voltage (in the order of $\mathrm{mV}$ ), especially for highly conducting sodium and hydrogen montmorillonite samples. The author concluded that the effect of dielectrophoresis on consolidated clay samples at the low voltages was negligible and not re-producible.

The importance of electrode insulation in dielectrophoresis experiment can be illustrated here. It is particularly critical in clay-water-electrolyte systems which is often highly conductive. On the other hand, dielectrophoresis requires relatively strong electric field intensity to obtain observable effectiveness, which means the requirement of high voltages. Pohl (1978) suggested that an electric field intensity of at least $500 \mathrm{~V} / \mathrm{m}$ is required in aqueous systems. Lockhart's experiment provided an experimental evidence toward these points. 


\subsection{Work of Inculet and Lo (1988)}

In their pioneer investigation, Inculet and Lo applied a non-uniform electric field (ac) on a natural clay block sample excavated from Wallaceburg, Ontario. The apparatus is shown in a schematic diagram of Fig.2.7(A). It consists of six insulated electrodes inserted through six $25.4 \mathrm{~mm}$ diameter holes pre-drilled into the $254 \mathrm{~mm}$ cubic block sample. This is typically an electrode-matrix for an approximate cylindrical field. It should be noted that an air space was maintained between the soil and the electrode. The electrode is composed of a copper rod, $4.8 \mathrm{~mm}$ in diameter and $300 \mathrm{~mm}$ in length, inserted in a plexiglas tube of $13 \mathrm{~mm}$ O.D. and $10 \mathrm{~mm}$ I.D., filled with transformer oil, as shown in Fig.2.8. The relative permittivity of the insulation layer is 3.4. The applied ac voltage $(60 \mathrm{~Hz})$ was $20 \mathrm{kV}$ between the high and ground electrodes.

Three tests were conducted by varying the depth of electrode insert in each test. The test duration was ranged from 26 to 28 days. During the test, the deformation of the soil block was observed through 8 settlement pins installed on the block surface, Fig.2.7(A). The water content and vane strength were tested before and after each test to determine the changes.

Water condensation was observed under the plexiglas cover of the soil block, at top of high voltage electrodes. Such water condensation was not observed in the control test. This observation again provides an indication that water migrated to the electrodes, the highest electric field intensity region, consistent with Lockhart's resilts. The authors concluded that the phenomenon of water condensation reflects the water movement in the soil mass towards the electrodes. 
The test results showed that the effectiveness was increased with the electrode depth, as shown in Table 2.2. In a cylindrical electric field, the field intensity is limited primarily in the cylindrical region defined by the length of electrode. Beyond the region, the field intensity is zero for an ideal cylindrical geometry. The boundary effect due to imperfection of the cylindrical geometry is negligible (Kraus and Carver, 1973). Hence in practical applications, electrodes should be installed to the depth where dielectrophoretic effect is required.

The measurement after treatment showed that for the electrode depth of $190.5 \mathrm{~mm}$, the vane strength of the clay increased from $225 \mathrm{kPa}$ to $240 \mathrm{kPa}$, with an increase of $966.7 \%$, turning the soft clay into a hard clay, Table 2.2 The corresponding water content was found decreased from $40.9 \%$ to $30.7 \%$, a decrease of $24.9 \%$.

As demonstrated in the experiment the properties of a natural clay changed dramatically upon applying a strong non-uniform electric field, which establishes the basis of applications in geotechnical engineering.

\subsection{Work of Lo, Ho and Inculet (1992)}

More recently, the same research group carried out a series of tests on tube samples of a sensitive soft clay from Gloucester, Ontario. The test apparatus is shown in Fig.2.7(B). The central high-voltage electrode, as described in previous section (Fig.28), and surrounding grounded electrodes provided a nearly perfect cylindrical electric field geometry. It should aleo be noted that the central electrode was installed in an air-dry hole of $25.4 \mathrm{~mm}$ in diameter. 
The primary variable in the experiment was the ac voltage at a constant frequency of $60 \mathrm{~Hz}$. The applied voltages were 10,18 and $25 \mathrm{kV}$, respectively. Each test lasted 21 days. The change of undrained shear strength and water content in relation to applied voltage is presented in Table 2.3. As seen the effectiveness of treatment was directly related to the applied voltage.

The major improvement of soil properties under the voltage of $18 \mathrm{kV}$ is summarized in Table 2.4. As seen that considerable increase in undrained shear strength and decrease in water content occurred in the slay samples after treatment. The general soil properties, such as stress-strain behaviour, brittleness index. undrained modulus and sensitivity of the soft sensitive clay are improved by the process. The pre-consolidation pressure of the Gloucester clay was increased after treatment which was assumed due to "over-consolidation" by the electric field, analogous to mechanical loadizg. It was found from chemical tests that the clay has increases in carbonate contents and salinity after treatment. The authors hence suggested that the bonding between soil particles was improved by the action of dielectrophoresis. The unchanged $\mathrm{pH}$ values indicated that there was no obvious decomposition of water molecules, and the adverse effect due to electrolysis was not evident. The experimental observations in this test series provide further evidence with respect to the effectiveness of dielectrophoresis on strengthening of soft clays. This technique may potentially become a powerful process for soil improvement. However, the authors indicated that most experimental results remained to be interpreted by means of the theory of dielectrophoresis and further research is necessary to advance the technique to field application. 


\subsubsection{Summary}

The experimental results reviewed in this section have provided some important evidences with respect to applications of dielectrophoresis in clay-waterelectrolyte systems. The migration of water towards the highest electric field intensity was noted in the synthetic kaolinite, synthetic montmorillonite and two natural clays from the experiments reviewed in this section, suggesting that negative dielectrophoresis has been taking place in these clay-water-electrolyte systems.

The promising potential of improving the engineering properties of soils by means of dielectrophoresis was also illustrated in these cases, especially in the works of Inculet and Lo (1988) and Lo et al (1992). 
TABLE 2.1 CONDUCTIVITY AND RELATIVE PERMITTIVITY OF VARIOUS MATERIALS (after Clark, 1966 and Kaye and Laby, 1986)

\begin{tabular}{lll}
\hline Material & Conductivity, S/m & Relative Permittivity, $\epsilon / \epsilon_{0}{ }^{+}$ \\
\hline Quartz & $10^{-18}-10^{-16}$ & $3 \cdot 4$ \\
Feldspar & $10^{-4}$ & 5 \\
Mica & $10^{-19}-10^{-15}$ & $6-7$ \\
Limestone & $10^{-5}$ & $10-15$ \\
sandstone & $10^{-3}$ & $6-15$ \\
Shale & $10^{-4}$ & $6-17$ \\
Sandy soil, dry & $10^{-9}-10^{-6}$ & $3-4$ \\
Clayey soil, dry & $10^{-4}-10^{-3}$ & $5-10$ \\
Deionized Water & $10^{-9}$ & -80 \\
Sea Water & 4 & $\sim 80$ \\
Plexiglas & $10^{-11}$ & $3-4$ \\
Transformer oil & $10^{-12}$ & 2.2 \\
Copper & $5.7 \times 10^{7}$ & $\infty$ \\
Aluminum & $3.5 \times 10^{7}$ & $\infty$ \\
Cast iron & $10^{6}$ & $\infty$ \\
Silicon Steel & $2 \times 10^{6}$ & $\infty$ \\
\hline
\end{tabular}

* $\epsilon_{0}=8.85 \times 10^{-12} \mathrm{~F} / \mathrm{m}$, permittivity of vacuum. 


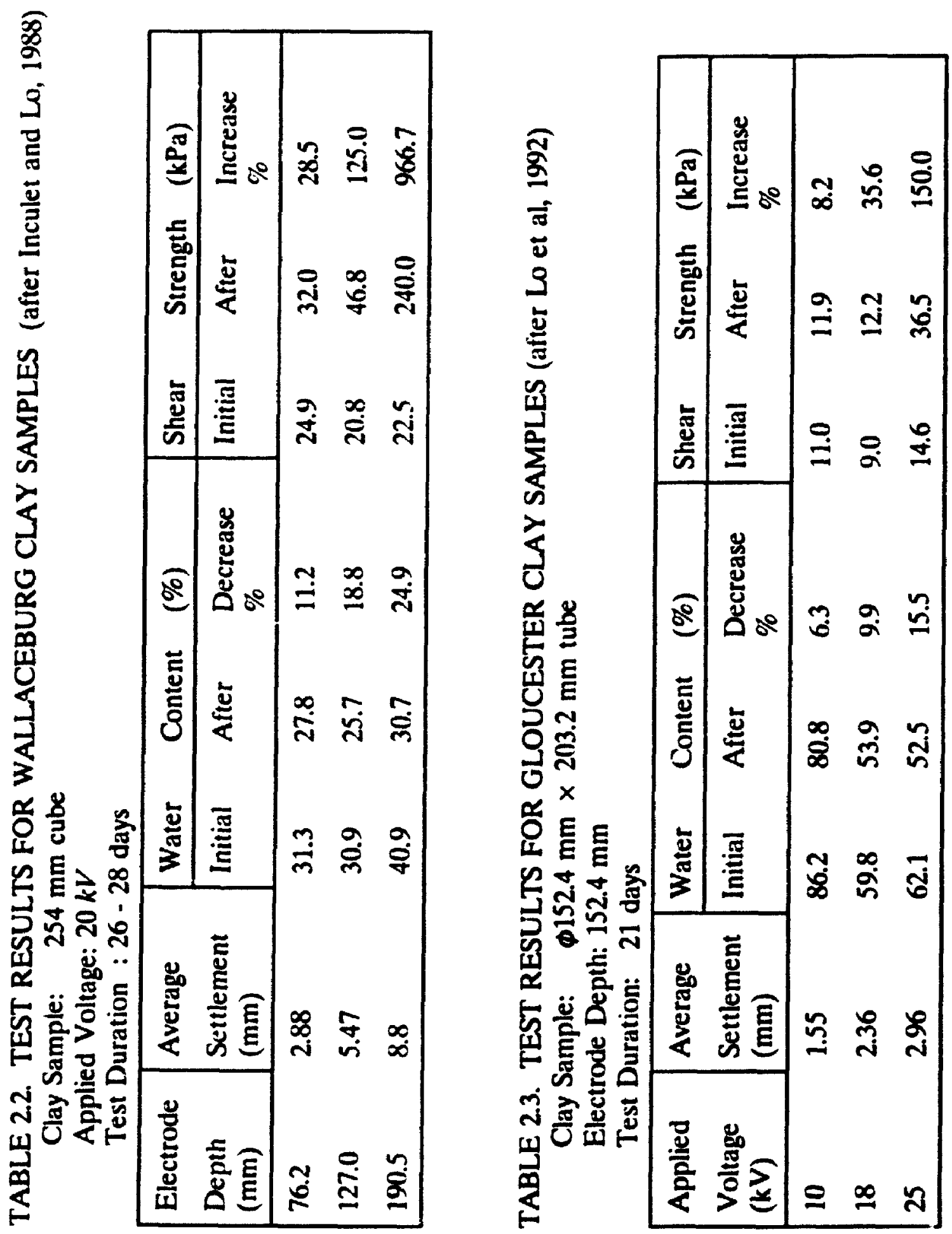


TABLE 2.4. SUMMARY OF DIELECTROPHORETIC TEST RESULTS FOR GLOUCESTER CLAY SAMPLES (after Lo et al, 1992)

Clay sample: $\Phi 152.4 \mathrm{~mm} \times 203.2 \mathrm{~mm}$ height (Shelby tube samples). Applied Voltage: $18 \mathrm{kV}$.

Electrode Depth: $152.4 \mathrm{~mm}$

Treatment Time : 21 days

\begin{tabular}{llll}
\hline Soil Property & Pre-Treatment & Post-Treatment & $\begin{array}{l}\text { Change } \\
\%\end{array}$ \\
\hline Settlement & - & $2.36 \mathrm{~mm}$ & - \\
Water content & $59.8 \%$ & $53.9 \%$ & -9.9 \\
Vane strength" & $9.0 \mathrm{kPa}$ & $12.2 \mathrm{kPa}$ & +35.6 \\
U/C strength & $15.9 \mathrm{kPa}$ & $21.0 \mathrm{kPa}$ & +32.1 \\
Failure strain* & $2.2 \%$ & $2.4 \%$ & +9.1 \\
Brittleness index & 0.36 & 0.21 & -41.7 \\
Undrained modulus* & $1660 \mathrm{kPa}$ & $2550 \mathrm{kPa}$ & +63.6 \\
Preconsolidation pressure@ & $43 \mathrm{kPa}$ & $62 \mathrm{kPa}$ & +44.2 \\
Sensitivity* & 20 & 10 & -50.0 \\
Plastic limit & $21.1 \%$ & $24.4 \%$ & +15.6 \\
Liquid limit & $44.3 \%$ & $49.8 \%$ & +12.4 \\
Soil pH value & 8.9 & 8.0 & -10.1 \\
Salinity & $1.37 \mathrm{~g} /$ & $2.25 \mathrm{~g} /$ & +64.2 \\
\hline
\end{tabular}

* Pocket vane test.

\# Unconfined compression test.

@ Consolidation test. 

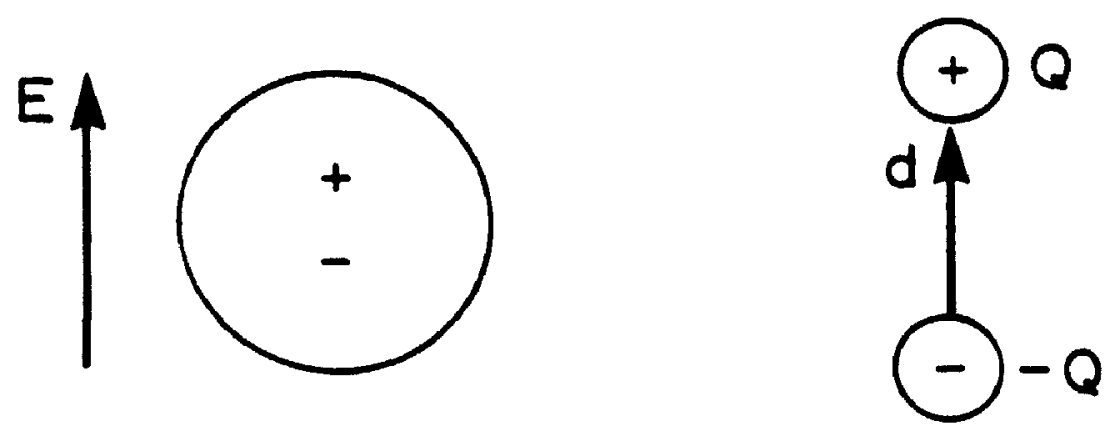

(a)

(b)

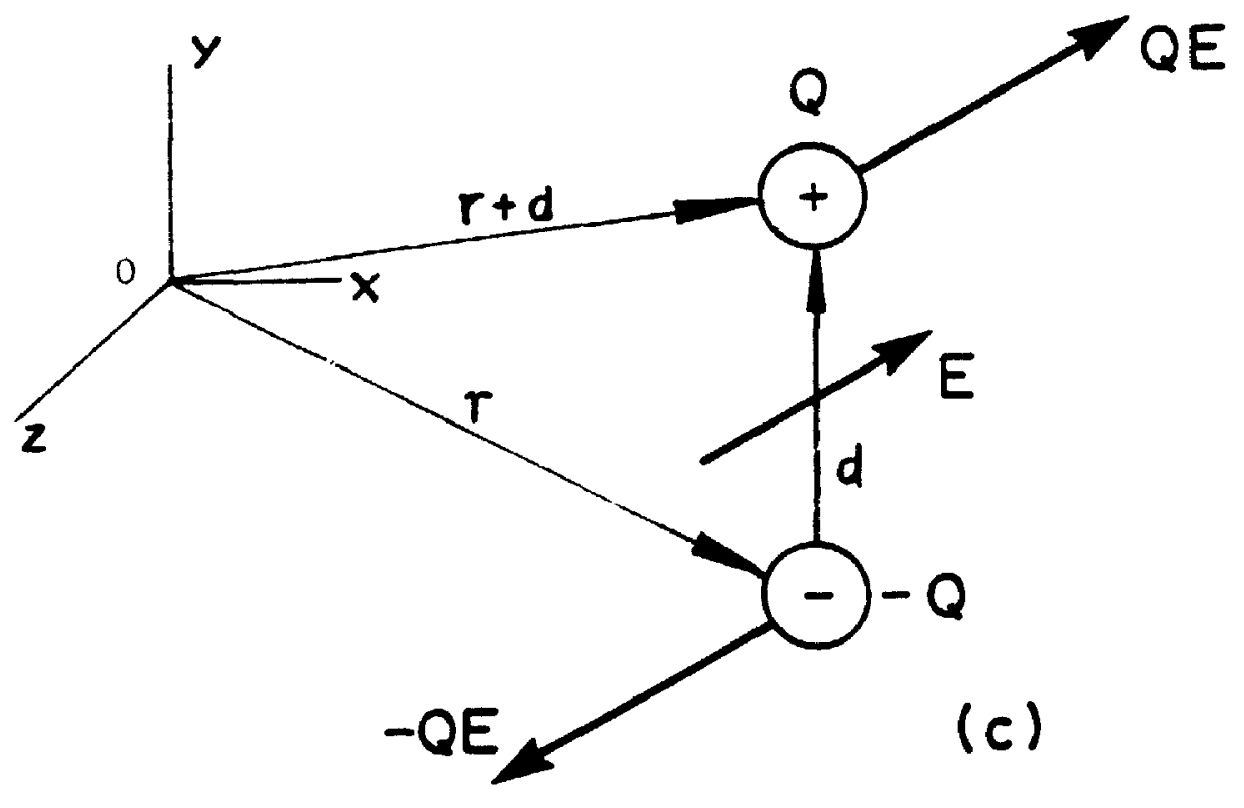

EIGUAE 2.1 (a) AN ELECTRIC DIPOLE (b) SCHEMATIC REPRESENTATION OF AN ELECTRIC DIPOLE (c) TORQUE ACTING ON AN ELECTRIC DIPOLE IN AN EXTERNAL ELECTRIC FIELD 


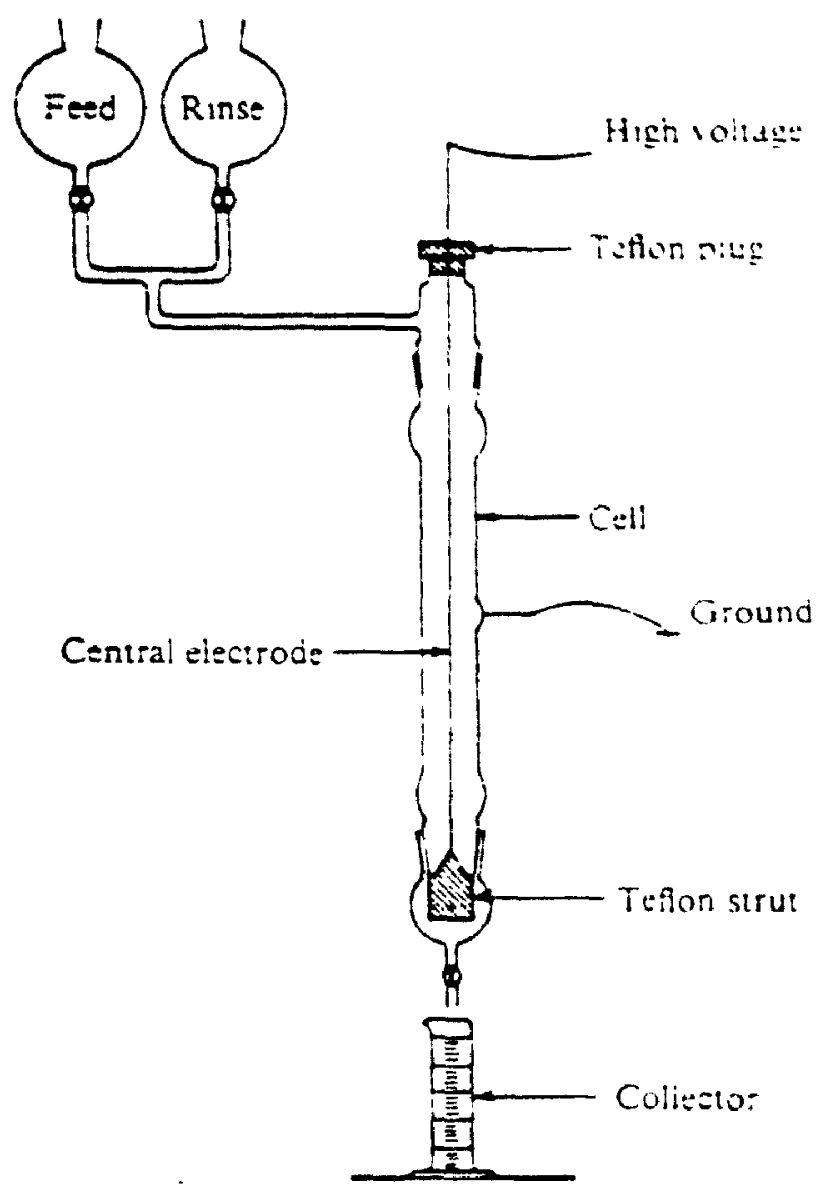

(a)

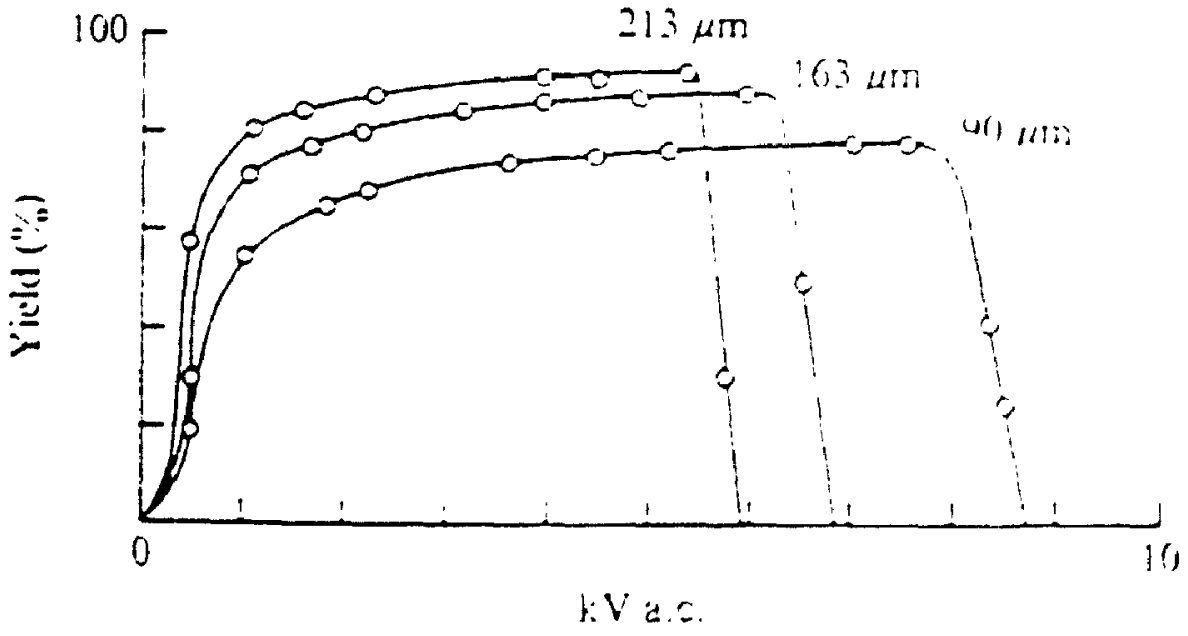

(b)

FIGURE 2.2 (a) DEP TEST CELL

(D) YTEED OF PAPTICLES (LEP FOPCE) $\because E$ APPIIED VOLTAGE

( AFTER POHL AND SCHWAR, 1959 ) 


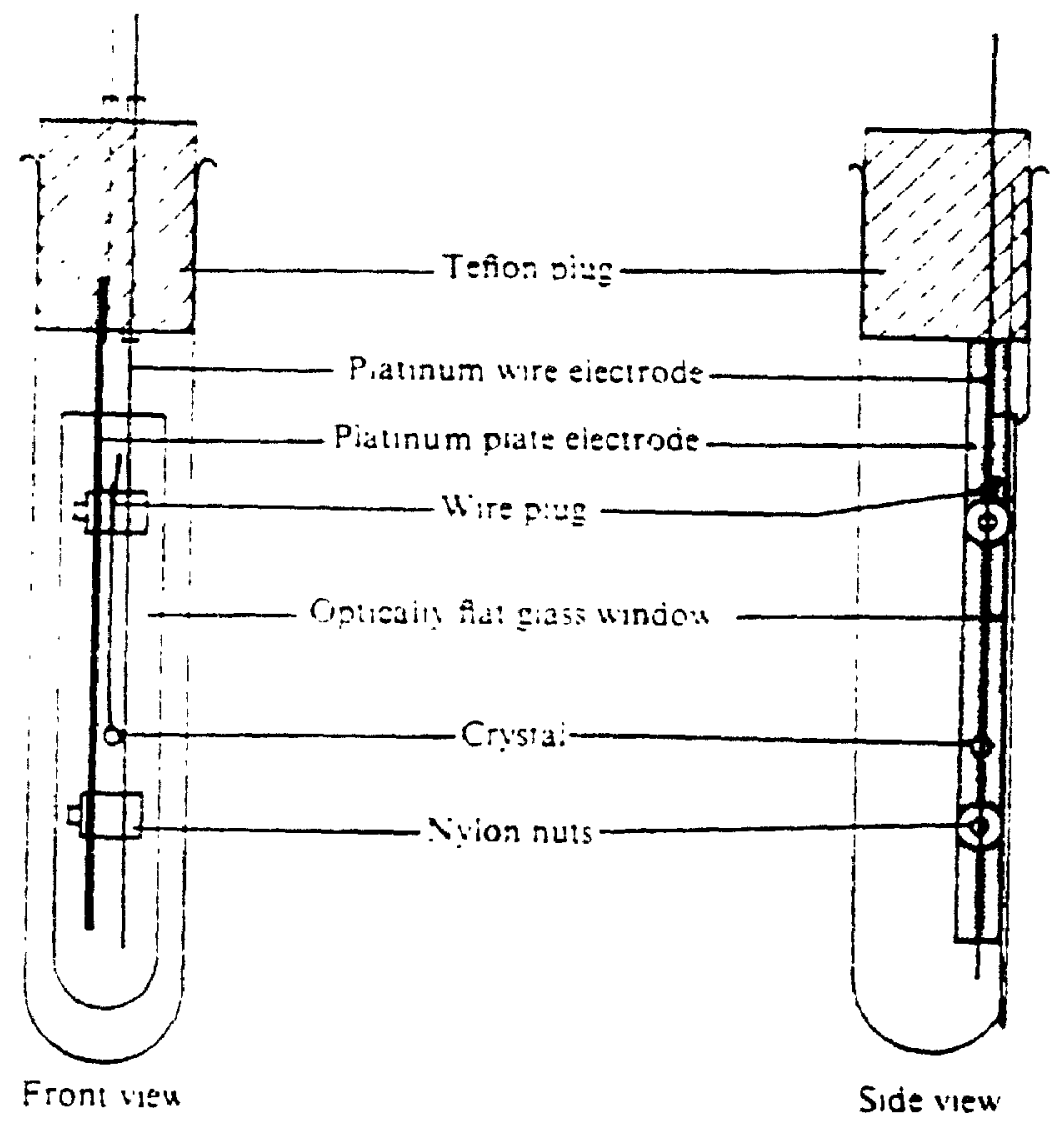

FIGURE 2.3 DEP TEST CELI FOR A SINGLE QUARTZ SPHERE (AFTER CHEN, 1967) 


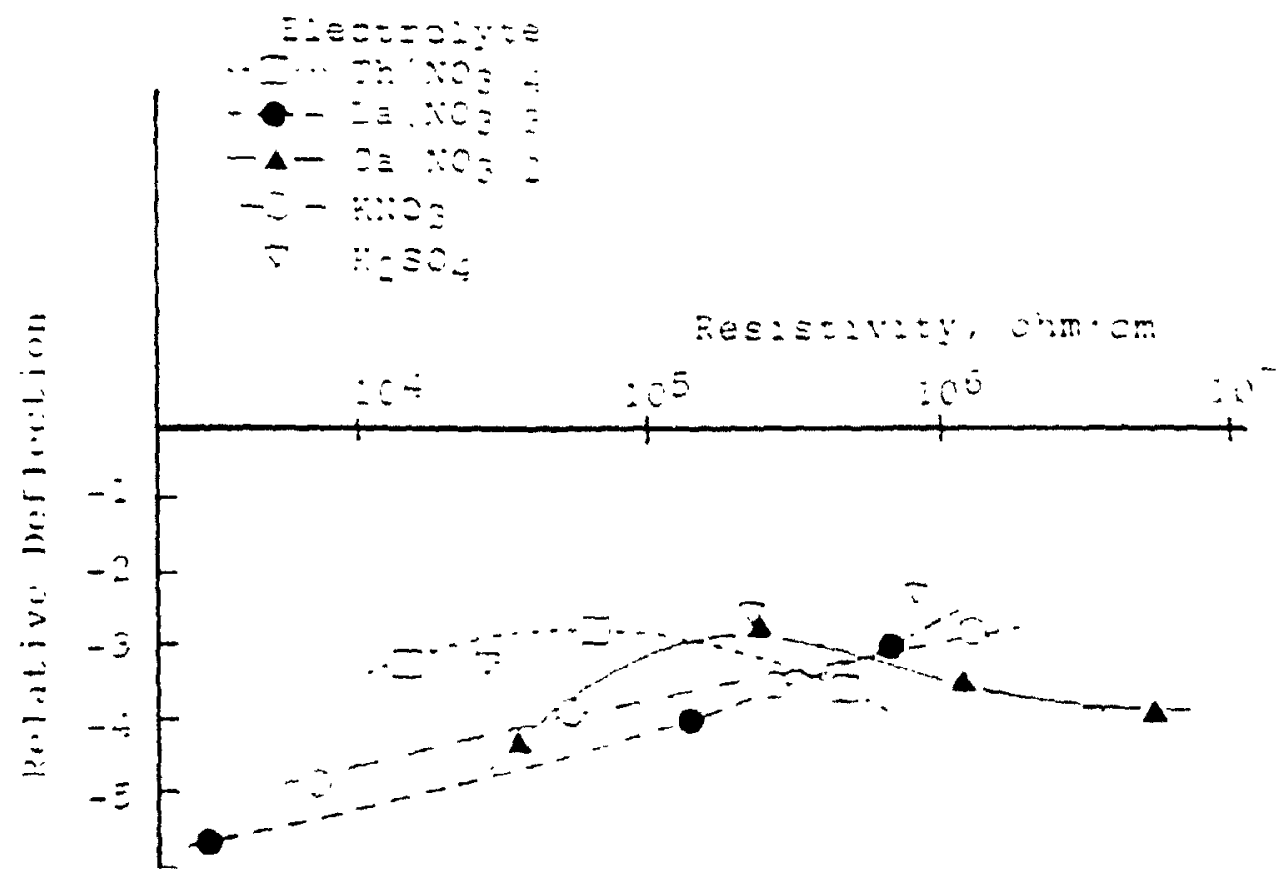

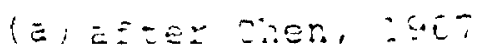

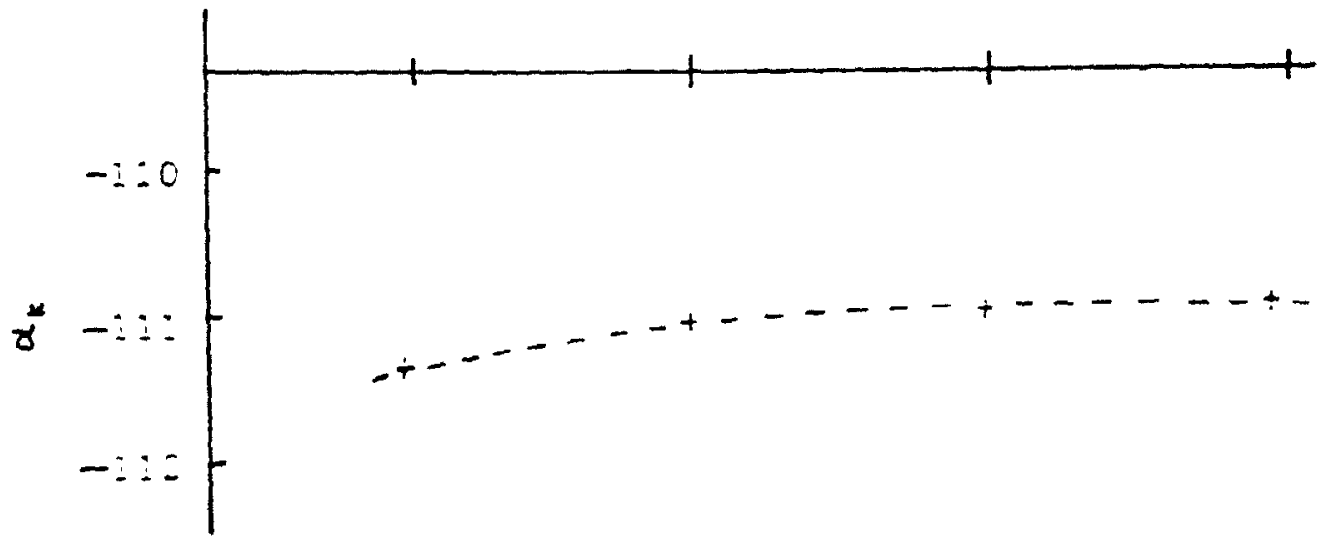

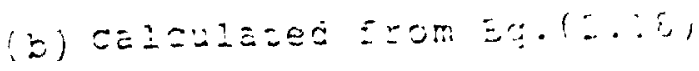

EIGURE 2.4 DIELECTROPHORESIS ON A SINGLE OUARTZ SPHEPE

(A) RESISTIVITY VS DEFLECTION: (DEF FORCE)

(B) RESISTIVITY VS RELATIVE FOLAPIZABIHIT? 


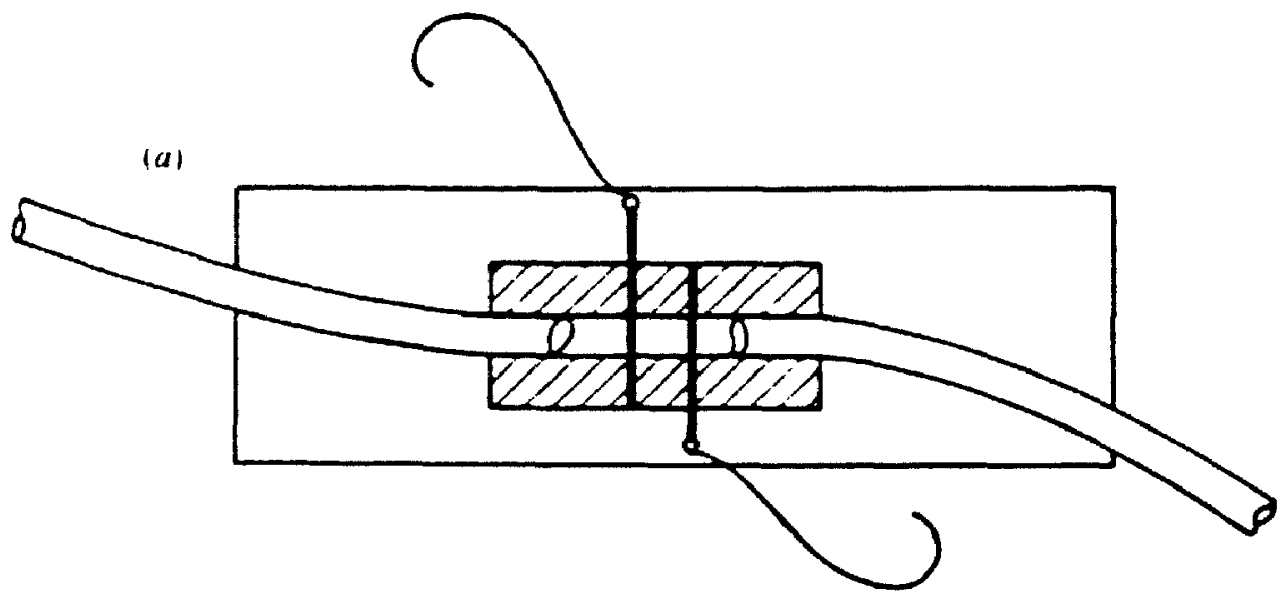

(b)

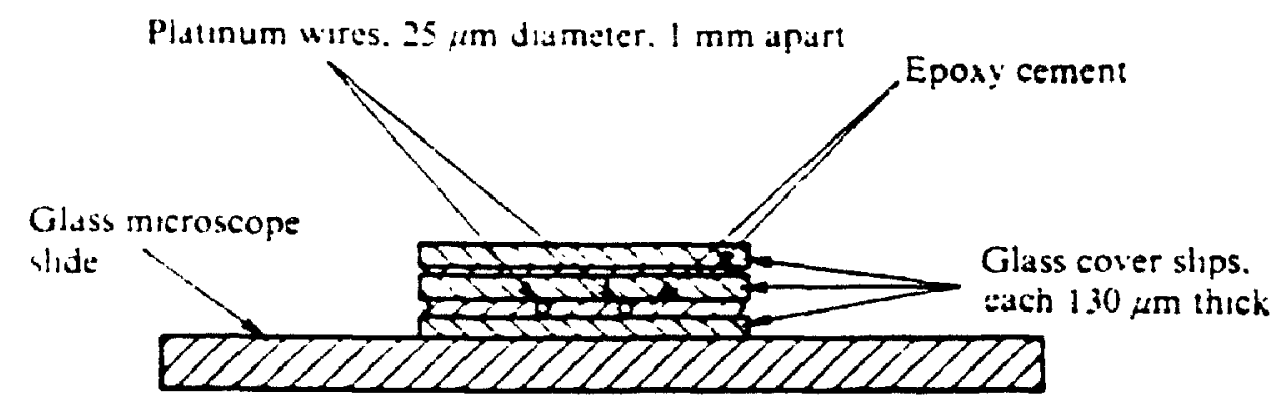

FIGURE 2.5 DEP TEST CELL FOR AQUEOUS COLLOID SILICA ( AFTER SCRIMAGER, 1974) 


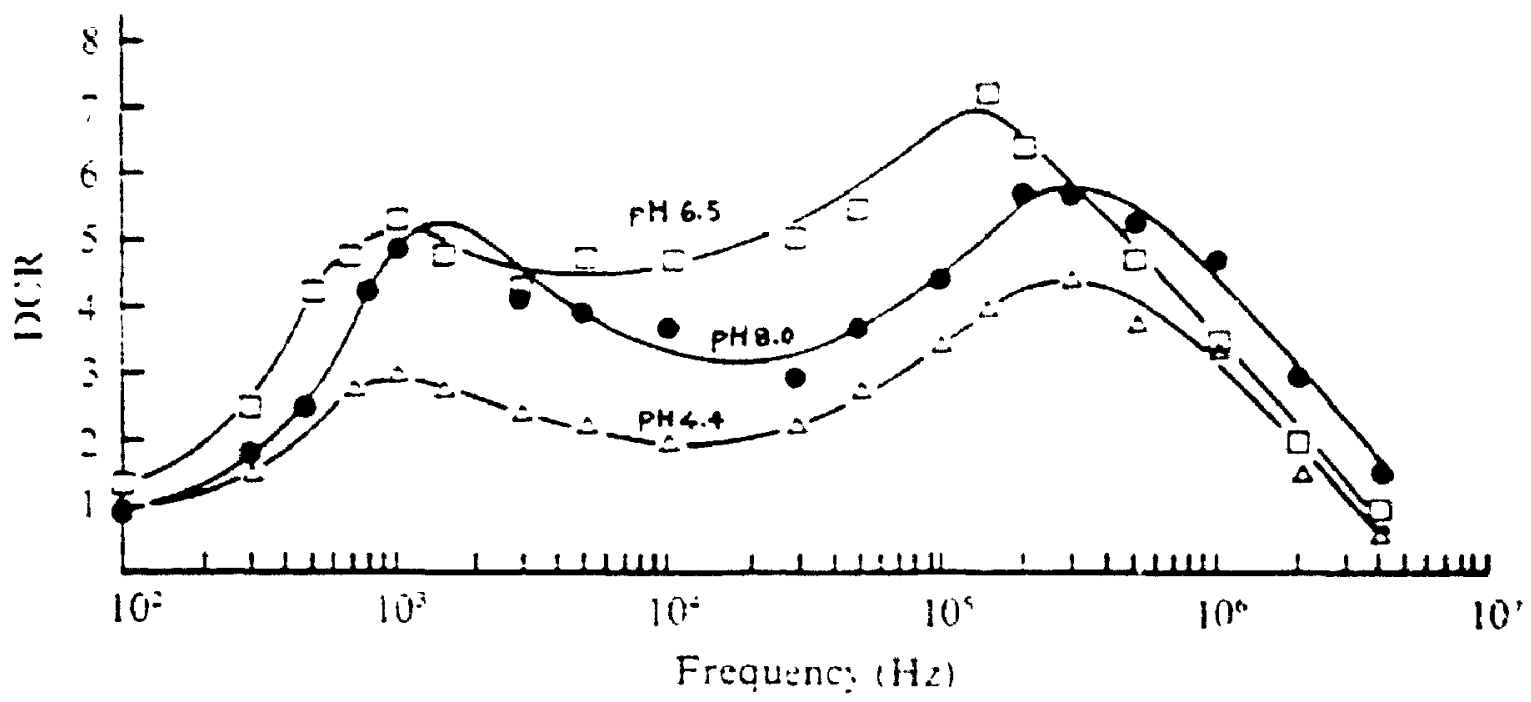

lai zEsect of pis

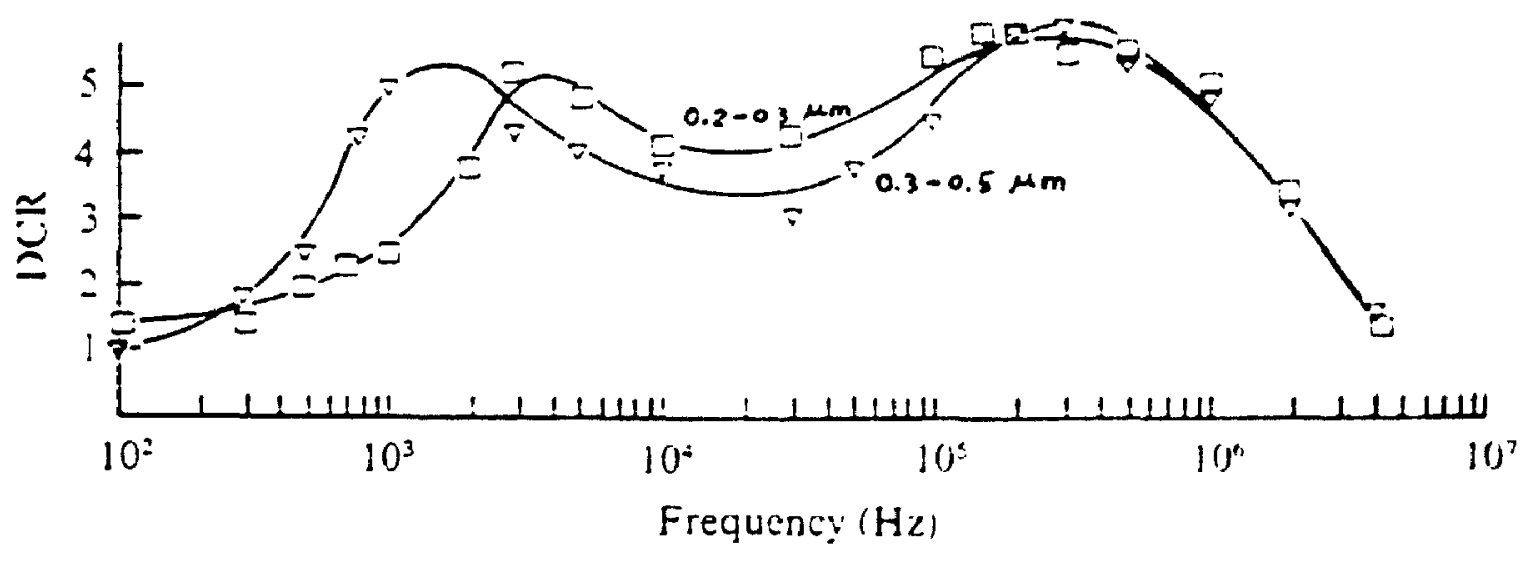

(b) Effect of pirtiale sizr

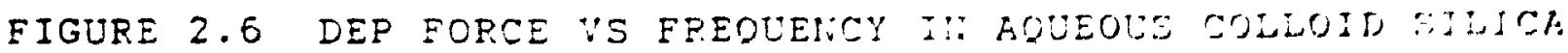
( AFTER POHL,:978) 

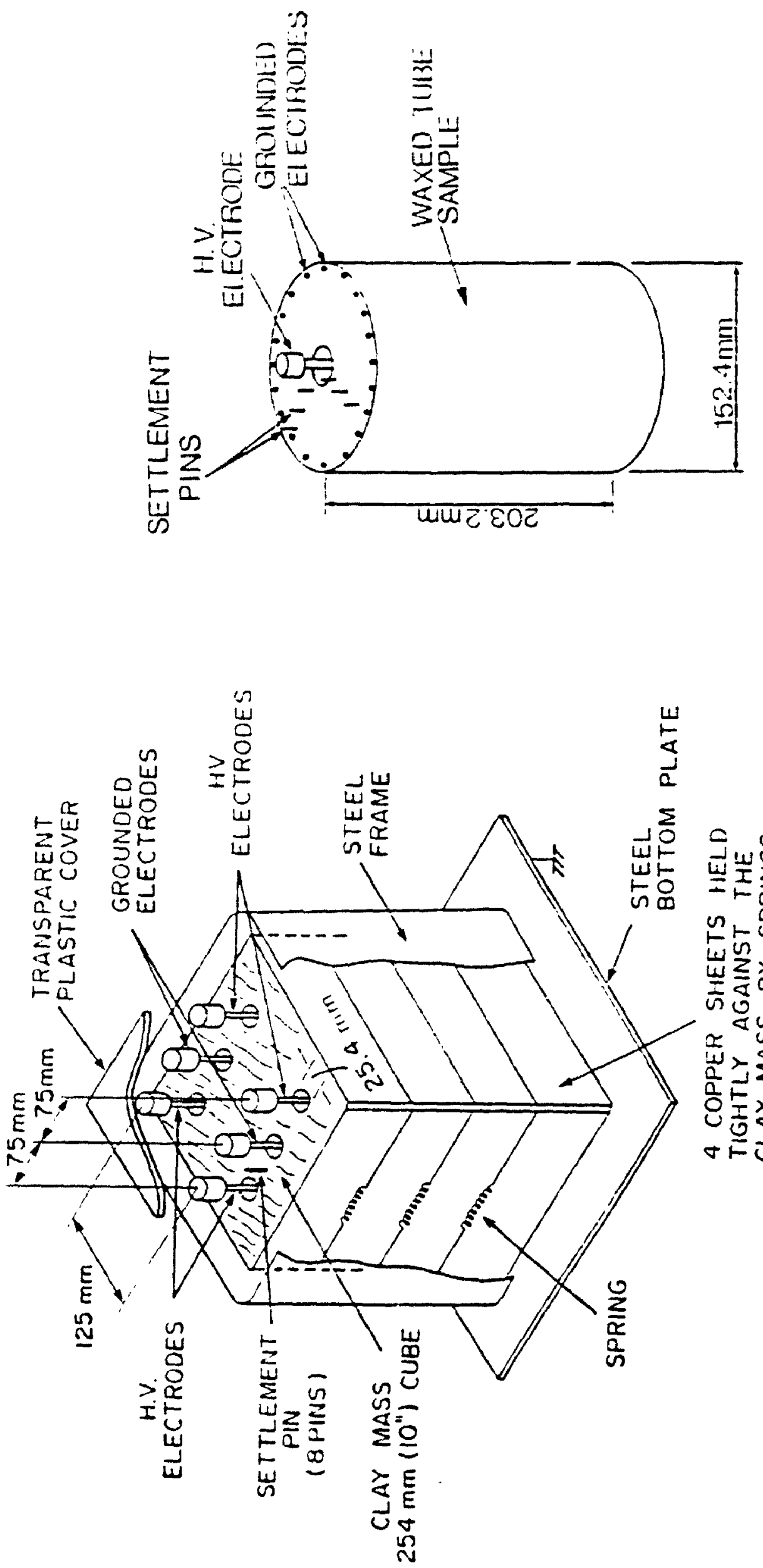

$\vdots$
$\simeq$
$\vdots$
$\bar{\Xi}$
$y$
0
0
0
0
0

$\Phi$

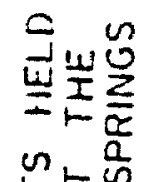

卢包

业市市

ज灾

它岛岕

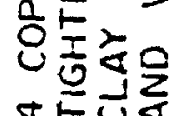

$\frac{2}{2}$

OFU⿺

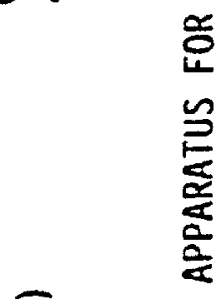

5

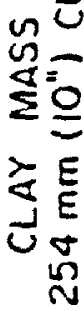




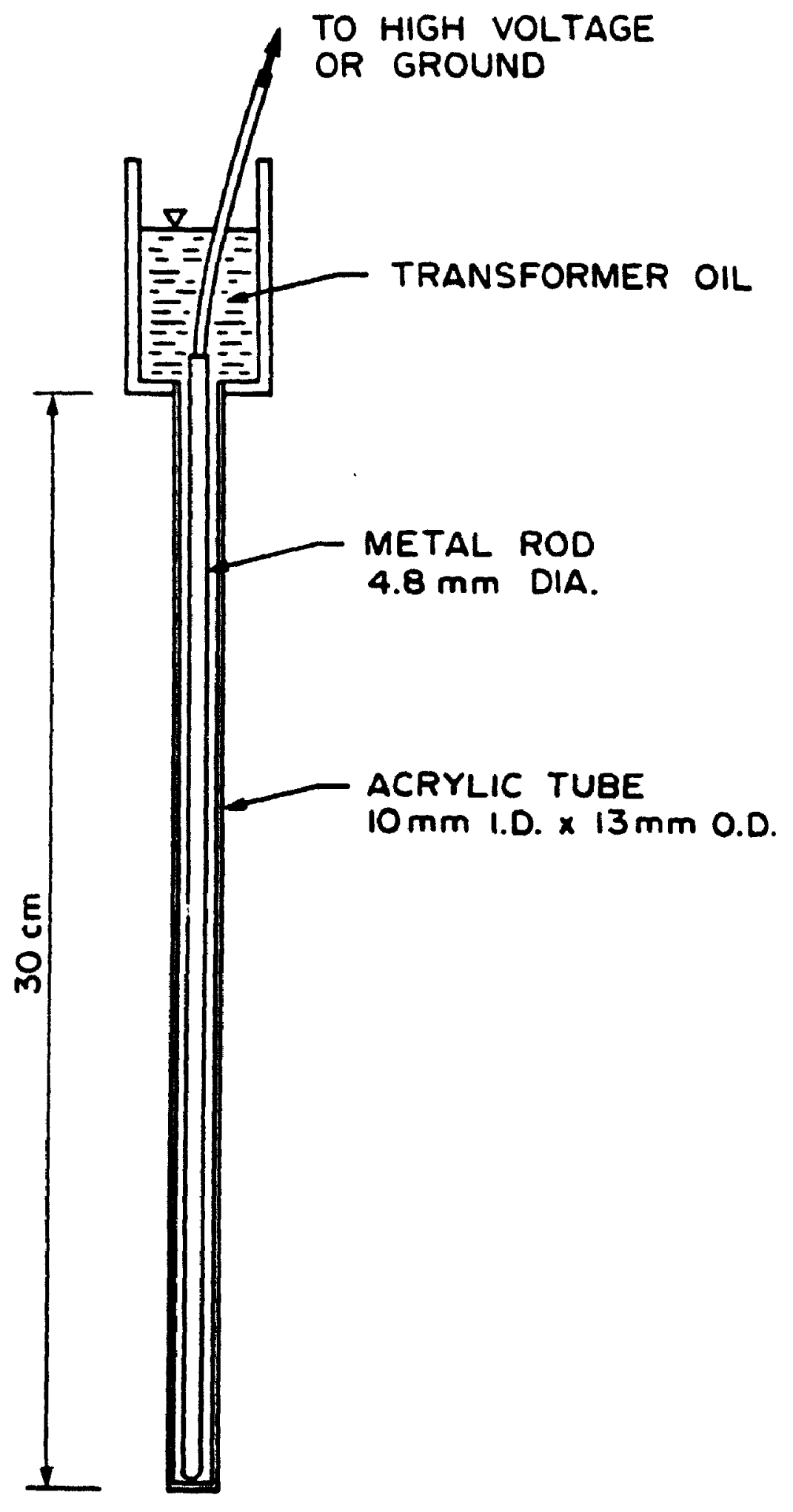

FIGURE 2.8 HIGH-VOLTAGE ELECTRODE (after Inculet and Lo, 1988) 


\section{CHAPTER 3}

\section{AN ANALYSIS IN DIELECTROPHORETIC FORCES}

\section{IN AQUEOUS COILOMAL SYSTEMS}

\subsection{Introduction}

The dielectrophoretic force as introduced in Chapter 2 (Eq.(28)) involves three basic terms: the volume of particle, the polarizability and the gradient of electric field intensity squared $\left(E^{2}\right)$. Since the volume of particles in a specific colloidal system is usually pre-determined, the other two terms are the key factors governing the dielectrophoretic force vector.

In modern terms, a colloidal suspension is defined as a system in which particles of colloidal dimensions (i.e. roughly between $1 \mathrm{~nm}$ and $1 \mu \mathrm{m}$ in at least one dimension of the particle) are dispersed in a continuous phase of a different composition ( van Olphen, 1977). A special case is the aqueous systems in which colloidal particles are suspended in water-electrolyte solutions. Clays as found in nature are classified as the typical aqueous colloidal systems in which colloidal sized clay particles act with each other in aqueous media (Mitchell, 1976).

The presence of electrical double layer in colloidal systems is well known. In 1879, H. von Helmholtz (Verwey, 1935) suggested that an electrical double layer is generally formed at the separation of two phases. He treated the problem mathematically by assuming the double layer to be virtually an electric condenser with two parallel plates separated by not more than a molecular distance. Since then the double layer theory has been developed and perfected by many scientists such as 
Gouy, Chapman and Stern (James and Parks, 1980). The applications of the double layer theory have been found in various areas, including those in soil science and geotechnical engineering (Mitchell, 1976, van Olphen. 1977).

The anomalously high permittivities in aqueous colloid suspensions were first reported in 1950's (Schwan, 1957). Schwarz (1962) carried on the exploration based on the experimental discovery and was the first one to interpret the phenomenon based on the theory of double layer. Dukhin and Shilov (1974) made the important contribution to expound the theory in their book (translated from Russian by D.Lederman, Israel Program for Scientific Transiations) titled Dielectric Phenomena and the Double Layer in Disperse Systems and Polyelectrolytes.

In order to understand the principles of dielectrophoresis in clays, the distribution of non-uniform electric fields required for the action of dielectrophoresis is discussed in this chapter. Then the dielectric behaviours of aqueous colloidal suspension systems are analyzed, starting from reviewing an experiment on dielectric behaviours of clay-water-electrolyte systems. The basic concept of electrical double layer and the theory of double layer polarization are introduced from both microscopic and macroscopic views. In order to describe quantitatively the dielectric properties of clay-water-electrolyte systems as influenced by the electrical double layer, the apparent permittivity is defined. Finally, the concept of effective polarizability is introduced to complete the theory of dielectrophoretic force in claywater-electrolyte systems. Based on the theory, the factors influencing the magnitude and direction of the dielectrophoretic force are discussed. 


\subsection{Non-Uniform Electric Field}

Dielectrophoresis is an effect existing in non-uniform electric fields only. The practical electrode configuration for geotechnical engineering purposes is one having cylindrical symmetry. Electrode arrangements which conform to this requirement include: a central electrode located coaxially within an outer cylindrical electrode; an electrode-pair comprised of two parallel electrodes, and an electrode-matrix comprised of a group of electrodes in parallel with one another. In the latter two arrangements, the cylindrical symmetry is only roughly approximated at the proximity of the electrode surface.

The distributions of cylindrical electric field with different electrode arrangements are discussed in this section as the basis for experimental aspects of dielectrophoresis.

\subsubsection{Composition of Electrode}

The composition of electrode is the primary considerations regarding to design of a dielectrophoresis experiment. The early work of Pohl and Schwar (1959) has provided some important facts. They found that at very small electrode diameter for certain applied voltage the motion of particles in a medium is either nil or so small as to be almost undetectable. This was attributed to the intense fiel.. with their corona-like discharge and the disruptive high conduction that followed.

The effect of insulation around the electrode was also discussed by the same authors. It was found that an insulation layer on electrodes had significantly accelerated the motion of particles. The elimination of electrochemical reactions on 
the electrode-suspension interface by applying a layer of insulation on the electrode surface was attributed to the effect. The insulation layer on electrode is of particular importance for systems in the conductive regime. The system would become overheated quickly as a result of conducting current for systems having linite conductivity, such as most water-electrolyte suspensions.

Based on these considerations, the high voltage electrode used in this study is consisted of a layer of insulation material surrounding the metallic conductor, as shown in Fig.2.8. The design has been proven successful in the early works of Inculet and Lo (1988) and Lo et al (1992) as reviewed in Chapter 2.

\subsubsection{Radial Flectric Field in Cylindrical Geometry}

The distribution of the electric field intensity can be derived accurately in cylindrical geometry. The analysis presented here is based on insulated central electrode, as shown in Fig.3.1.

Consider a radial electric field in the volume between a central electrode (a metallic rod of radius $R_{a}$ insulation layer of radius $R_{b}$ and permittivity $\epsilon_{b}$ ), and an outer electrode of radius $\mathbf{R}_{c}$. The annular space between the central and an outer electrode is filled with a material of permittivity $\epsilon_{\mathrm{m}}$. The perfect cylindrical symmetry is only valid when the length of the electrodes, 1 , is much larger than the separation distance, $\mathbf{R}_{c}-\mathbf{R}_{\mathbf{g}}$, between the central and outer electrode.

Suppose an ac voltage $U_{0}$ is applied on the central electrode and the outer electrode is grounded. The electric field intensity distribution can be represented in the cylindrical coordinates as 


$$
E=\left\{\begin{array}{cl}
0 & \left(0 \leq r<R_{a}\right) \\
\frac{U_{o} r_{o}}{\epsilon_{b} \lambda r} & \left(R_{a} \leq r<R_{b}\right) \\
\frac{U_{o} r_{b}}{\epsilon_{m} \lambda r} & \left(R_{b} \leq r<R_{c}\right) \\
0 & \left(r \geq R_{c}\right)
\end{array}\right.
$$

where

$$
\lambda=\frac{1}{\epsilon_{b}} \ln \frac{R_{b}}{R_{a}}+\frac{1}{\epsilon_{m}} \ln \frac{R_{c}}{R_{b}}
$$

E: electric field intensity (vector) (volt/m):

$\mathrm{U}_{0}$ : potential difference between the electrode-pair (volt);

$\mathrm{r}$ distance to the centre of coordinates. (m);

$\mathbf{r}_{\mathbf{0}}$ : unit radial vector:

$\epsilon_{\mathrm{h}}: \quad$ permittivity of insulator. $(\mathrm{F} / \mathrm{m})$

$\epsilon_{\mathrm{m}}$ : permittivity of the material filled in the annular space between $R_{b}$ and $R_{c}(\mathrm{~F} / \mathrm{m})$

A detailed derivation of Eqs.(3.1) and (3.2) is enclosed in Appendix 1.

We should note that $\lambda$ is only related to the geometry of the field and the dielectric properties of materials involved. In this study. the radius of electrode conductor rod $R_{a}$, the radius $R_{b}$ and permittivity $\epsilon_{b}$ of the insulation layer are predetermined in the electrode design. the material filled in the annular space is an 
aqueous clay suspension, hence $K_{m}=80$, thu ' he radius of outer electrode $R_{c}$ is the only variable governing the parameter $\lambda$. i.e.. $\lambda$ is solely controlled by the geometry of electric field.

Eq.(3.1) indicates that the field intensity in a cylindrical electric field is proportional to the potential difference $U_{0}$ and inversely proportional to the radius $r$. The direction of the field is along $r_{0}$ i.e., along the radial direction of the cylindrical coordinates. The effect of material filled in the annular space hetween the inner and outer electrodes is represented by the permittivity of the material in the electric field, $\epsilon_{\mathrm{m}}$. The parameter $\lambda$ is determined by geometry of electric field. Note that the electric field intensity is discontinuous at the interface of the insulation layer and the material filled in the electric field, i.e., at $r=R_{b}$, Fig.3.1.

\subsubsection{Gradient of $E^{2}$}

The dielectrophoretic force is proportional to term $\nabla E^{2}$, Eq. (2.8). For a perfect cylindrical symmetry, the term $\nabla E^{2}$ in the annular space $\left(R_{c}<r<R_{h}\right)$ is

$$
\begin{aligned}
\nabla E^{2} & =r_{\bullet} \frac{\partial E^{2}}{\partial r}+\theta_{\bullet} \frac{1}{r} \frac{\partial E^{2}}{\partial \theta} \\
& =-r_{\bullet} \frac{2 U_{0}^{2}}{e_{m}^{2} \lambda^{2} r^{3}}\left(V^{2} / m^{3}\right)
\end{aligned}
$$

where $r_{0}$ and $\theta_{0}$ are the unit radial vector and unit tangential vector in the cylindrical coordinates, respectively.

Substitute Eq.(3.3) into Eq.(2.8), the dielectrophoretic force can be expressed 
as

$$
F=-\alpha v r_{\cdot} \frac{U_{o}^{2}}{e_{m}^{2} \lambda^{2} r^{3}} \quad\left(R_{a}<r<R_{b}\right)
$$

As described in Eq.(3.4), in a perfect cylindrical symmetry electric field induced by a potential difference (voltage) $\mathbf{U}_{0}$

(1) The dielectrophoretic force varies inversely as the third power of the spacial function $r$, which results in a rather drastic decrease of the force with increasing radius $r$;

(2) The dielectrophoretic force is proportional to the square of the potential difference, $U_{0}$. It implies that dielectrophoresis requires a rather high potential difference for strong effects;

(3) The negative radial unit vector $\boldsymbol{r}_{\mathrm{o}}$ in Eq. (3.4) indicates that the direction of dielectrophoretic force is always towards the central axis of the cylindrical coordinates, i.e., towards the region of higher field intensity. Thus the direction of dielectrophoretic force can be changed only by the polarizability $a$ of the system, as we discussed in Chapter 2;

(4) The combined effect of dielectric properties of the system and the geometry of the electric field is represented by the term $\left(\epsilon_{\mathrm{m}}^{2} \lambda^{2}\right)$, to which the dielectrophoretic force is inversely proportional.

\subsubsection{Equi-Intensity Lines}

In order to describe the nature of non-uniform electric field, the concept of 
equi-intensity lines is herein introduced. An equi-intensity line is defined as the traces of the equal absolute values of electric field intensity. From Eq.(3.1), in a perfect cylindrical field at radius $\mathbf{R}$, the absolute values of the electric field intensity are constant:

$$
E=|E(R)|=\frac{U_{0}}{\varepsilon_{m} \lambda R}
$$

Thus the equi-intensity lines are the concentric circles surrounding the central electrode, as shown in Fig.3.2.

It should be emphasized that only the absolute values of the electric field intensity are equal on an equi-intensity line. The direction of electric field intensity is represented by field-lines in electromagnetics (Kraus et al, 1973), which is different from the equi-intensity lines discussed here. The field lines in a perfect cylindrical electric field are along the radial direction, as shown in Fig.3.2.

As a convention, the value marked on each equi-intensity line represents the field intensity in percentage comparing to that at the interface of the insulation layer and the material filled in the electric field, i.e.,

$$
v_{E I I}=\frac{E(r)}{E\left(R_{b}\right)} \times 100 \%
$$

where $v_{E I L}$ is the value marked on the equi-intensity line, $E(r)$ is the absolute value of field intensity at radius $r$, and $E\left(R_{b}\right)$ is that at radius $R_{b}$, i.e., at the surface of insulation layer, as shown in Fig. 3.1. 
Since dielectrophoretic force is independent of the direction of electric field, the equi-intensity line is a convenient way to illustrate the magnitude distribution of non-uniform electric fields for studying dielectrophoresis.

\subsubsection{Electrode-Pair}

For geotechnical engineering applications, the ideal cylindrical field is not practical because an outer electrode with cylindrical shape is difficult to manage. The most convenient way to create a non-uniform electric field distribution is to use an electrode-pair. As shown in Fig.3.3, an electrode-pair is readily constituted by two electrodes arranged in parallel. The centre-to-centre distance between the electrodes is $2 \mathrm{~s}$. A fixed potential difference $U_{o}$ is maintained between the electrodes thus the charge on the electrodes is equal in magnitude but opposite in sign.

The components of electric field intensity in the $\mathbf{x}$ and $\mathbf{y}$ directions are

$$
\begin{aligned}
E_{x} & =-\frac{\partial U}{\partial x} \\
& =-\frac{t_{e}}{2 \lambda e_{m}}\left[\frac{x+s}{(x+s)^{2}+y^{2}}-\frac{x-s}{(x-s)^{2}+y^{2}}\right] \\
E_{y} & =-\frac{\partial U}{\partial y} \\
& =-\frac{U_{o} y}{2 \lambda e_{m}}\left[\frac{1}{(x+s)^{2}+y^{2}}-\frac{1}{(x-s)^{2}+y^{2}}\right]
\end{aligned}
$$

where 


$$
\begin{aligned}
& U_{\mathrm{o}}=\text { potential difference between the eiectrode-parr: } \\
& \epsilon_{\mathrm{m}}=\text { permittivity of material filled in the electric field: } \\
& \Lambda=\text { parameter determined by Eq.(3.2) in which } R_{c}=s .
\end{aligned}
$$

The absolute value of the electric tield intensity is

$$
|E|=\sqrt{E_{x}^{2}+E_{y}^{2}}
$$

where $E_{x}$ and $E_{y}$ are given by (3.7) and (3.8) respectively. Eys.(3.7) and (3.8) are derived in detail in Appendix 1.

The distribution of the absolute values of electric field intensity may be represented as equi-intensity lines in the plane perpendicular to the axis of the electrode-pair, as shown in Fig.3.4, in which (a) and (b) are two electrode configurations to be used in the experimental program. The values on the equiintensity lines are the field intensity in percentage normalized to the value at the surface of electrode, $E\left(R_{b}\right)$. It may be seen from Fig. 3.4 that the electric field decreases drastically with the distance away from the electrode and the cylindrical symmetry may be approximated only at proximity of the electrode surface. At the region between the electrode-pair, the field intensity is high in value but has lower gradient comparing to the surrounding area.

\subsubsection{Electrode-Matrix}

Electrode-matrix is another convenient way to create a non-uniform electric field. The arrangement of electrode can be rather arbitrary, as long as the distances between electrodes are much less than the length of electrodes so the assumption of 
infinite length electrode is valid. An electrode-matrix used in this study is a central electrode with higher potenti-i surrounded by four electrodes of equal potential at lower value, as shown in Fig.3.5. The potential difference between the central electrode and any of the surrounding electrode is $U_{0}$.

The components of electric field intensity along $x$ and $y$ directions are

$$
\begin{aligned}
E_{x} & =-\frac{\partial U}{\partial x} \\
& =\frac{U_{0}}{2 \lambda e_{m}}\left(\frac{x}{r_{0}^{2}}-\frac{x+d}{r_{1}^{2}}-\frac{x+d}{r_{2}^{2}}-\frac{x-d}{r_{3}^{2}}-\frac{x-d}{r_{4}^{2}}\right) \\
E_{y} & =-\frac{\partial U}{\partial y} \\
& =\frac{U_{0}}{2 \lambda e_{m}}\left(\frac{y}{r_{0}^{2}}-\frac{y-d}{r_{1}^{2}}-\frac{y+d}{r_{2}^{2}}-\frac{y+d}{r_{3}^{2}}-\frac{y-d}{r_{4}^{2}}\right)
\end{aligned}
$$

where

$$
\begin{aligned}
& U_{0}=\text { potential difference between the central and surrounding electrodes; } \\
& \Lambda=\text { parameter determined from Eq.(3.2) with } R_{c}=s ; \\
& \epsilon_{\mathrm{m}}=\text { permittivity of material filled in the electric field; } \\
& r_{0}, r_{1}, r_{2}, r_{3}, r_{4}=\text { distances from } P(x, y) \text { to the electrodes, respectively, as shown }
\end{aligned}
$$

in Fig.3.5:

$$
\begin{aligned}
& r_{0}=\left(x^{2}+y^{2}\right)^{1 / 2} ; \\
& r_{1}=\left[(x+d)^{2}+(y-d)^{2}\right]^{1 / 2} ; \\
& r_{2}=\left[(x+d)^{2}+(y+d)^{2}\right]^{1 / 2} ;
\end{aligned}
$$


$r_{3}=\left[(x-d)^{2}+(y+d)^{2}\right]^{1 / 2}$

$r_{4}=\left[(x-d)^{2}+(y-d)^{2}\right]^{1 / 2} ;$

$r_{-} \rightarrow \infty$, distance to the potential reference;

$d=s / 2, s=$ distance between the central and surrounding electrodes, Fig.3.5.

The absolute value of the electric field intensity is then

$$
|E|=\sqrt{E_{x}^{2}+E_{y}^{2}}
$$

where $E_{x}$ and $E_{\gamma}$ are derived from Eq.(3.10) and Eq.(3.11), respectively.

The distribution of electric field intensity for the electrode-matrix is illustrated by the equi-intensity lines in Fig. 3.6. It may be seen that the cylindricil symmetry is approximated in the vicinity of each electrode with regions of lower field intensity between every two surrounding electrodes.

\subsubsection{Summary}

This section covered the determination and the distribution of the non-uniform electric field. The analysis of a practical electric field configuration for dielectrophoretic water extraction is based on cylindrical geometries in various alternative arrangements, such as:

- a central electrode coaxial with an outer cylindrical electrode;

- two parallel electrodes;

- a matrix of parallel electrodes with arbitrary distances between them.

The analysis leads to the following conclusions:

(1). A relatively high potential difference should be applied to generate the 
necessary non-uniform electric field for dielectrophoresis;

(2). The electrodes must be insulated electrically by a layer of insulation material over the entire surface in contact with the soil;

(3). The distance between electrodes should be much less than the length of the electrodes to provide valid cylindrical field symmetry;

(4). The concept of equi-intensity lines is introduced to help visualize the nonuniformity of the electric fields and estimate the dielectrophoretic forces;

(5). The distributions of equi-intensity lines for three geometric arrangements, later used in experiments, have been determined.

\subsection{Dielectric Phenomena in Aqueous Colloidal Systems}

\subsubsection{Clay-water-electrolyte System}

A clay-water-electrolyte system in colloid chemistry is defined as colloidal clay particles suspended in an aqueous solution. On thie other side, a clay mass in nature is almost always in the form of a solid that can sustain shear stress. In soil mechanics, macroscopically, a natural clay is treated as a continuum, although it is composed of discrete clay particles suspended in an aqueous medium. Considering the views of the two disciplines, a clay-water-electrolyte system in this study is defined as a clay suspension in which the clay particles are sedimented and consolidated to form a primarily liquid-in-solid, not solid-in-liquid systcm. In such a system, both the colloid chemistry and the soil mechanics knowledge may apply. This concept has been commonly adapted by most authors (e.g. Lee, 1968; Mitchell, 1976; Sparks, 1986 etc.). 


\subsubsection{Experiments of Arulanandan and Mitchell (1968)}

The dielectric propertues. i.e. permittivity and conductivity, of the kaolinitewater-electrolyte systems were tested under various conditions. such as temperature. particle size, pore fluid cation type and pore electrolyte concentration. The particle size of the kaolinite clay ranged from $0.65 \mu \mathrm{m}$ to $10 \mu \mathrm{m}$. The specimens were prepared by sedimenting and consolidating the slurries of the kaolinite suspension to a water content of $64 \%$ to $75 \%$. The dimensions of the specimens were $35 \mathrm{~mm}$ in diameter and $76-127 \mathrm{~mm}$ in height.

The permittivity and conductivity of the specimens were determined by means of the impedances measured on a Wheatstone ac bridge. The test cell wis composed of two flat circular platinum electrodes with adjustable separation distance to fit in specimens having different heights. The frequency of measurement ranged from $30 \mathrm{~Hz}$ to $10^{5} \mathrm{~Hz}$.

The dielectric properties of the kaolinite-water-electrolyte systems at low frequency (in the order of $100 \mathrm{~Hz}$ ) are of particular interest of this study. As summarized in Table 3.1:

(1). The conductivity of the kaolinite systems was in the order of $0.1-1 \mathrm{~S} / \mathrm{m}$;

(2). The relative permittivity of the kaolinite systems was in the order $10^{4} 1010^{5}$;

(3). Both permittivity and conductivity of the kaolinite systems were increased by raising the temperature, Table 3.1a:

(4). The larger particle size resulted in increase of permittivity, whereas the conductivity was not affected, Table $3.1 \mathrm{~b}$;

(5). Both the permittivity and the conductivity of the kaolinite systems increased at 
higher electrolyte concentration, Table 3.1c;

(6). The effect of cation type on permittivity and conductivity appeared to have diverging trends, Table $3.1 \mathrm{~d}$.

(7). The conductivity of the clay-electrolyte-water system is lower than that of the pore fluid, Table $3.1 \mathrm{c}$ and $3.1 \mathrm{~d}$.

The relative permittivity of dry kaolinite is 7.65 (Clark, 1966). The experiment revealed that at presence of water, the permittivity of the kaolinite-water-electrolyte systems was astonishingly high, suggesting that the surface properties play a dominant role to the dielectric behaviour. It will be presented in later sections that the theory of double layer polarization may provide a successful interpretation to the phenomenon.

\subsubsection{Electrical Double Layer}

The commonly accepted double layer theory for clay-water-electrolyte systems can be described in the so-called Gouy-Stern model (van Olphen, 1977).

Clay particles are negatively charged mostly because of isomorphous substitution. (e.g., of a small amount of $\mathrm{Al}^{3+}$ for $\mathrm{Si}^{1+}$ of the kaolinite) The charge is balanced by exchangeable cations adsorbed to the clay cnycal surfaces. The internal balance of charges is incorporated in the concept of the electrical double layer. The general distribution of ions adjacent to a clay surface is depicted in Fig. 3.7(A). The double layer consists of a fixed part (the Stern layer) and a diffuse part (the Gouy layer) where the balancing cations are held by electrostatic attraction. In the double layer, an equilibrium is maintained between the negative surface charge and an 
equivalent amount of positive ionic charge which is accumulated in the liquid near the particle surface.

The Stern layer is only a molecule in thickness. In this layer, the electric potential drops linearly with distance, from a value $\Phi_{0}$ at the particle surface to a value $\Phi_{d}$ which is called the Stern potential. A low permittivity $(K-3-6)$ is assigned to the me-jum within the Stern layer. In his calculation, Stern accounted for the finite size of ions and the possibility of their specific adsorption and assumed that the surface charge is balanced by the charge in solution which is distributed as the Stern layer to a distance $d$ from the surface and a diffuse layer which has a Boltzman distribution thereafter, as described later.

The negative surface charge density at the particle surface, $\rho$, is in equilibrium with that in the Stern layer and the Gouy layer

$$
p=-\left(p_{1}+p_{2}\right)
$$

$\rho_{1}$ is the charge in the Stern layer and $\rho_{2}$ in the Gouy layer.

By an approximate statistical treatment, considering the number of positions of the cations in the Stern layer and in solution, the charge density in the Stern layer is (Sparks, 1986)

$$
\rho_{1}=\frac{N_{1} z F}{1+\frac{N_{A} v}{M c} \exp \left[-\frac{2 F \Phi_{d}+\phi}{R T}\right]}
$$


here

$\rho_{1}=$ Stern layer charge density $\left(\mathrm{C} / \mathrm{m}^{2}\right)$.

$N_{i}=$ Number of adsorption sites available per $m^{2}$ of surface to ionic species $i$.

$N_{A}=$ Avogadro's Number $=6.02 \times 10^{23}$.

$M=$ Molar mass of the solvent ( $\mathrm{kg}$ ).

$v$ = Solvent density $\left(\mathrm{kg} / \mathrm{m}^{3}\right)$.

$c=$ Molar concentration of electrolyte $\left(\mathrm{mol} / \mathrm{m}^{3}\right)$.

$\Phi$ = Specific adsorption potential (J).

$\Phi_{d}=$ Stern potential (V)

d = Thickness of Stern layer (m).

$z=$ Ionic valence.

$\mathrm{F}=$ Faraday constant $=9.6487 \times 10^{4} \mathrm{C} / \mathrm{mol}$.

$R=$ Gas constant $=8.314 \mathrm{~J} /(\mathrm{mol} \cdot \mathrm{K})$.

$T=$ Absolure temperature $(K)$.

Since a linear drop in potential across the Stern layer is assumed, the surface charge is given by the Gauss equation for a molecular condenser:

$$
p=\frac{e_{d}}{d}\left(\Phi_{0}-\Phi_{d}\right)
$$

here, $\epsilon_{d}$ is the average permittivity of the Stern layer.

Beyond the molecular condenser, the ions in the solution are distributed as a diffuse atmosphere in the Gouy layer. At equilibrium between any point in the double layer and the bulk solution for any ionic specie, the molar concentration of 
the ions can be described by Boltzman's equation (Sparks, 1986):

$$
c=c_{\bullet} \exp \left(-\frac{z F \Phi}{R T}\right)
$$

where $c$ is the local molar concentration of the ions, $c_{0}$ is its molar concentration far away from the surface in the equilibrium liquid and $\Phi$ is the electrical potential in volt. The other symbols are the same as defined early. Thus when equilibrium is established in the double layer, the average local concentration of ions can be expressed as a function of the potential: $\Phi$, at that distance.

In the Gouy layer, the electric potential is related to the distance to the particle surface, $x$; and the charge density $\rho_{2}$ is, by the Poisson equation (Sparks, 1986),

$$
\frac{d^{2} \Phi}{d x^{2}}=-\frac{p_{2}}{e}
$$

where $\epsilon$ is the permittivity of the solution.

The solution of Poisson equation (3.17) can be obtained as the distribution of the electrical potential in relation to distance $x$ from the particle surface (Sparks, 1986): 


$$
\frac{z F \Phi}{R T}=2 \ln \frac{e^{x x}+\tanh \frac{z F \Phi_{d}}{4 R T}}{e^{x x}-\tanh \frac{z F \Phi_{d}}{4 R T}}
$$

where

$$
x=\left(\frac{2 z^{2} F^{2} c_{o}}{\epsilon R T}\right)^{\frac{1}{2}} \quad\left(m^{-i}\right)
$$

the other symbols are as defined befure.

Eq.(3.18) is the Boltzman distribution expressing the electrical potential as a function of distance from the Stern potential, $\Phi_{d}$, in the diffuse Gouy layer. It describes the decay of potential with distance at a given Stern potential, electrolyte concenuration anc, for a given ion-type. This decay is approximately exponential, as slown in Fig. 3.7(B).

The charge in the Guay ditfuse layer may be solved from Poisson equation (3.17) and ti.a Boltzman distribution (3.16), (Sparks, 1986)

$$
\rho_{2}=\sqrt{8 c \varepsilon R T} \sinh \frac{z F \Phi_{d}}{2 R T}
$$

where the symbols have the same meaning and value as given before.

The Stern layer and the Gouy layer together as the Gouy-Stern model of elecurical double ayer give a complete description to the distribution of ions 
surrounding a charged particle. The double layer potential distribution of the GouyStern model is represented in Fig. 3.7(B).

The particle surface potential $\Phi_{0}$, the Stern potential $\Phi_{d}$, the Stern layer charge $\rho_{1}$ and the Gouy layer charge $\rho_{2}$ can be computed from Eqs. (3.13), (3.14), (3.15) and (3.20). The distribution of electrical potential in the double layer can be obtained from the Boltzman distribution Eq.(3.18) after knowing the surface potential and the Stern potential. An example calculation for a kaolinite-waterelectrolyte system reviewed in Section 3.3.2 is enclosed in Appendix 2. The following points obtained from the kaolinite-water-electrolyte systems are of general interest for understanding the problem:

(1). The ratio of the Stern layer charge and the Gouy layer charge

$$
\rho_{1} / \rho_{2}=0.231 \mathrm{C} / \mathrm{m}^{2} / 0.092 \mathrm{C} / \mathrm{m}^{2}=2.5
$$

indicating that a large fraction of cations are located on the kaolinite particle surface, as suggested in the Gouy-Stern model;

(2). The ratio of the surface potential and the Stern layer potential

$$
\Phi_{\mathrm{o}} / \Phi_{\mathrm{d}}=3.17 \text { volt } / 0.139 \text { volt }=22.8
$$

indicating a rapid potential drop in the Stern layer, which is about $0.5 \mathrm{~nm}$ in thickness;

(3). The Stern potential is in the order of $10^{2} \mathrm{mV}$ for the kaolinite system. 


\subsubsection{Zeta Potential}

An important concept in electrochemistry and colloid chemistry is the zeta potential. By definition, the zeta potential is the electrical potential in the double layer located at the shearing plane between the ibulk water and an envelope of water which moves with the particle (van Olphen, 1977). The zeta potential is computed according to mobility of colloidal particles in electrochemistry (Bockris and Reddy, 1970). It is known that the zeta potential is not equal to the surface potential $\Phi_{0}$, but it is to some extent, comparable with the Stern potential $\Phi_{d}$. The schematic location of zeta potential in a double layer is marked in Fig. 3.7(B).

\subsubsection{Polarization of Double Layer and Apparent Permittivity}

Many scientists have attempted to explain the very high values of permittivity in aqueous colloidal systems (Schwarz, 1962; Dukhin and Shilov, 1974; Shilov and Rozen, 1975; Pohl, 1978; etc). Among them, the approach of Dukhin and Shilov (1974) is considered most rigorous. They analyzed the behaviours of both the Stern layer and the Gouy layer and found that the polarization of the Stern layer is overshadowed by the Gouy layer. Therefore the polarization of the Gouy diffuse layer is a major factor which determines the permittivity. The polarization of the diffuse layer may be illustrated in a simplified sketch as shown in Fig.3.8. The quantitative derivation is very complicated which requires simultaneous solution of some twentieth equations. The close-form solution of these equations gives the 
increment of permittivity due to double layer polarization in an ac field. A simplified expression for the increment at low frequency in dilute solution for the case of a univalent electrolyte is given by

$$
\begin{aligned}
\Delta K & =K_{a}-K_{p} \\
& =\frac{9}{4} p K_{m}\left[3 m \exp \left(\frac{F \Phi_{q}}{2 R T}\right)+\exp \left(\frac{F \Phi_{d}}{2 R T}\right)\right]^{2}
\end{aligned}
$$

where

$\Delta K=$ increment of relative permittivity of the colloidal system;

$\mathrm{K}_{\mathbf{a}}=$ apparent relative permittivity of the colloidal system due to double layer polarization;

$\mathbf{K}_{\mathbf{p}}$ = intrinsic relative permittivity of the particle;

$p=$ volume fraction of the colloidal particles in the solution,

$$
p=\frac{1}{1+G_{s} w}
$$

in which $G$, is the specific gravity of particle and $w$ is the water content of the colloidal system;

$\mathbf{K}_{\mathrm{m}}=$ relative permittivity of the medium solution;

$\Phi_{\zeta}=$ Zeta potential $(\mathrm{V})$;

$\Phi_{\mathbf{d}}=$ Stern potential $(\mathbf{V})$

$\mathrm{m}=$ Dimensionless constant; $\mathrm{m}=0.1$ for aqueous suspensions.

The other symbols are as defir $\approx d$ before. 
It is shown that an aqueous colloidal system will generate an increment of permittivity relative to the intrinsic permittivity of the particles in dry state. The increment is affected by the volume fraction of the particles in the system, temperature, electrolyte concentration and type, and surface charge of particles. The increment of permittivity can be computed once these factors are determined.

In the expression of Eq.(3.21), Dukhin and Shilov emphasized the distinction between the zeta potential and the Stern potential. In their calculations, however, they assumed them to be nearly equal. It should also be noted that there is an important assumption in the Dukhin-Shilov theory: the colloidal system is considered as dilute, i.e. the volume fraction of the system, $p$, is much less than 1.

The analysis r, Dukhin and Shilov is basically from microscopic view where the molecular behaviours of aqueous colloid systems were considered. Experiments have shown 'scellent agreement with the theory (Shilov and Rozen, 1975; Pohl, 1978).

Pohl (1978) further gave a qualitative interpretation from a macroscopic view to help the understanding of the phenomenon. As discussed in Chapter 2, the dielectric properties of matter can be represented as a complex permittivity, $\epsilon^{*}$, Fq.(2.13). The absolute value of the complex permittivity, which is the portion seen in an experiment as an in-phase permittivity of the system, is given by

$$
\left|e^{*}\right|=\left[e^{2}+\left(\frac{o}{\omega}\right)^{2}\right]^{\frac{1}{2}}
$$


The conductive term in Eq.(3.23) increases without limit as the frequency approaches zero, provided that the ac conductivity $\sigma$ does not approach zero too rapidly in this limit. This actually takes place upon polarization of double layer. The net result is an unusual and very large intensification of the signal of the particle polarization, which can be tracked back to the conductivity component $\sigma$ in Eq.(3.23) at low frequencies.

The concept of apparent permittivity may be introduced in order to described the double layer polarization discussed in Section 3.3.5. In aqueous colloid systems, charges accumulate at the particle-medium interface to form the electrical double layer. At very low frequencies the charges easily follow the alternating field and contribute to the polarization mechanism. The polarization of particles by their intrinsic mechanism is almost totally overshadowed by polarization of the charges. This appears to the external observers as a huge polarization leading to a giant permittivity. Therefore at low frequency the charges in the double layer would largely control the permittivity measurement.

By adopting the theory of Dukhin and Shilov, an apparent relative permittivity may be defined as

$$
\begin{aligned}
K_{c} & =K_{p}+\Delta K \\
& =K_{p}+\frac{9}{4} p K_{m}\left(3 m \exp \left(\frac{F \Phi_{c}}{2 R T}\right)+\exp \left(\frac{F \Phi_{d}}{2 R T}\right)^{2}\right.
\end{aligned}
$$

where $K_{2}$ is the apparent relative permittivity of clay particles with surrounding electric double layer; $K_{p}$ is the intrinsic relative permittivity of particles at dry utate 
and $\Delta \mathrm{K}$ is the increment of relative permittivity caused by polarization of double layer. The other symbols have been defined before.

It is seen that the value of $K_{\mathbf{a}}$ is controlled by the volume fraction of particle; the temperature; the Stern potential; and the zeta potential.

In order to examine the agreement between the theoretical apparent permittivity and experimental facts, the apparent permittivity of a kaolinite-waterelectrolyte system is computed following the double layer calculation for the same system. The Stern potential of the kaolinite system at temperature $293 \mathrm{~K}$ is 0.139 volt for the electrolyte $0.01 \mathrm{~N} \mathrm{NaCl}$, as calculated in Appendix 2. The zeta potential of the system is assumed equal to the Stern potential. The water content is $47.5 \%$. By assuming a reasonable specific gravity for kaolinite as 2.7 , we obtain the volume fraction of particle as 0.44 from Eq.(3.22). It is known that the waterelectrolyte has a relative permittivity of approximately 80 . Substituting these values into Eq.(3.24) we obtain

$$
K_{2}=2.82 \times 10^{4}
$$

The relative permittivity measured by Arulanandan and Mitchell is $2.7 \times 10^{4}$ (Table 3.1c). We may see that the agreement is very good considering the approximations made during computation and possible experimental error. It is noted that although the intrinsic permittivity of particle $K_{p}$ appears in Eq.(3.24), it is totally overshadowed by the increment $\Delta \mathrm{K}$ as the latter is about 4 orders higher in magnitude. 
Table 3.2 lists the apparent relative permittivities of three common clay minerals in $0.01 \mathrm{~N} \mathrm{NaCl}$ solution at temperature of $293 \mathrm{~K}(20 \mathrm{C})$, calculated based on the typical C.E.C values and the specific surface areas of these minerals. It may be seen that tt.. relative apparent permittivities are in the order of $10^{3}$ to $10^{4}$. comparing to the relative permittivities of water $(\sim 80)$ and dry clay minerals $(5 \sim 10)$, Table 2.1. It may also be seen that in general the minerals having higher surface surcharge (e.g., kaolinite) will demonstrate higher apparent permittivity than the minerals with lower surface charge (e.g. montmorillonite) in electrolyte-water.

The effects of the soil water content and clay mineral type to the apparent permittivity may be illustrated in Fig.3.9. It is known that the apparent permittivity is proportional to the particle volume fraction, Eq.(3.24) which in turn may be converted to the soil water content by Eq.(3.22). We may see that the apparent permittivity increases with decreased soil water content. However, it should be emphasized here that this trend may not be extended to the soils of lower water content ( $w<60 \%$ typically), as the assumption in Eq.(3.21) is no longer valid when the particle volume fraction is comparable to 1 .

\subsubsection{Conduction of Clay-Water-Electrolyte System}

The conductivity of dry clay soils is in the order of $10^{-4}$ to $10^{-3} \mathrm{~S} / \mathrm{m}$, Table 2.1. However, the conductivity of a clay-water-electrolyte system can be many times higher $t$ presence of water-electrolyte as the conduction of a clay-water-electrolyte system is controlled by aqueous electrolytic conduction via pores and along clay particle 
surfaces but negligibly through the silicate network.

The ions which conduct the current result from the dissociation of salts when the salts are dissolved in water. Since each ion is able to carry only a definite quantity of charge, the more ions that are available in a solution and the faster they travel, the greater the charge that can be carried. Hence, the solution with the larger number of ions will have the higher conductivity. Thus, in general, a clay soil which contains saline water within its pores will have a greater conductivity when the salinity of the water is high than when it is low; salinity is a major factor in determining the conductivity of a clay soil.

On the other hand, clay particles act as a separate conducting path in addition to the electrolyte path. The conductivity of this added path is lower than the electrolyte path. The clay mineral conductivity lies in Gouy diffuse layer of the double layer as the cations in the diffuse layer are free to move under the influence of an applied electric field. The conductivity of a clay-water-electrolyte system as observed from experiment, therefore, is the total effects of the electrolyte conduction and clay particle surface conduction.

\subsection{7. ummary}

The dielectric phenomena in aqueous colloidal suspensions are discussed in this section. The observed anomalously high permittivity in these systems has been successfully interpreted by the theory of double layer polarization. The concept of apparent permittivity is introduced based on the theoretical exploration and experimental results. The conduction behaviour of clay-water-electrolyte systems are 
dominated by the salinity of pore water-electrolyte. Soft to firm clays encountered in nature can be treated generally as the clay-water-electrolyte systems in which the principles discussed in this chapter may apply.

\subsection{Effective Polarizability}

\subsubsection{Definition}

The effective polarizability may be introduced by adapting the concepts of apparent permittivity and apparent conductivity in clay-water-electrolyte systems. In the definition of polarizability, Eq.(2.18), let the apparent permittivity $\epsilon_{2}$ replace the intrinsic permittivity of particles, $\epsilon_{\mathrm{p}}$, we obtain the following expression:

$$
\alpha_{e f f}=3 e_{m}\left(\frac{\omega^{2} \tau_{e f f}^{2}}{1+\omega^{2} \tau_{e f f}^{2}} \frac{e_{a}-e_{m}}{e_{a}+2 e_{m}}+\frac{1}{1+\omega^{2} \tau_{e f f}^{2}} \frac{\sigma_{p}-\sigma_{m}}{\sigma_{p}+2 \sigma_{m}}\right)
$$

where

$a_{\text {eff: }}$ effective polarizability of the particle-electrolyte-water system;

$\epsilon_{\mathrm{a}}$ : apparent permittivity;

$T_{\text {eff }}$ effective relaxation time,

$$
\tau_{e f f}=\frac{e_{a}+2 e_{m}}{\sigma_{p}+2 \sigma_{m}}
$$

The other symbols are as defined before.

The polarizabiiity has the unit of $F / m$, the same as that of permittivity. Thus we may introduce a relative effective polarizability, defined as 


$$
\alpha_{\Sigma \phi}=\frac{\alpha_{e f}}{\epsilon_{0}}
$$

The relative effective polarizability is sometimes more convenient in practical applications.

Finally, we may obtain the corresponding expression of dielectrophoretic force from: Eq.(2.8) as

$$
\begin{aligned}
F & =\frac{1}{2} \alpha_{\Delta \sigma} \vee \nabla E^{2} \\
& =\frac{1}{2} \alpha_{R \theta f} \epsilon_{0} \vee \nabla E^{2}
\end{aligned}
$$

in which all symbols are as defined before.

\subsubsection{Factors Influencing Effective Polarizability}

Similar as the classic theory reviewed in Chapter 2, the effective polarizability may be categorized in three characteristic regimes, depending on the effective relaxation time of a clay-water-electrolyte system in an external field with constant angular frequency $(\omega)$ :

(1) Dielectric regime.

In a high frequency electric field with non-conductive particles and medium, we have 


$$
\omega \tau_{e f}>>1
$$

then the system is said in the dielectric regime, in which the effective polarizability approaches the limit as $\omega r_{\text {eff }} \rightarrow \infty$ :

$$
\alpha_{\infty}=3 e_{m} \frac{e_{a}-e_{m}}{e_{a}+2 e_{m}}
$$

It will be shown later that the dielectric regime does not exist for clay-waterelectrolyte systems in low frequency electric field $(\omega<1000 \mathrm{~Hz})$.

(2) Conductive regime.

Most Clay-water-electrolyte systems are located in the conductive regime when a low frequency electric field is applied. A system is considered in a conductive regime at the limit of

$$
\omega \tau_{e f f}<<1
$$

as $\omega r_{\text {efr }} \rightarrow 0$, the polarizability approaches to

$$
\alpha_{\phi t}=3 e_{m} \frac{\sigma_{p}-\sigma_{m}}{\sigma_{p}+2 \sigma_{m}}
$$

Eq.(3.32) shows that in the conductive regime, the polarizability is dominated by the conductivities of the particle and medium as well as the permittivity of the medium, and is not affected by the apparent permittivity of the particles. 
(3) Transient regime.

Clay-water-electrolyte systems with low salinity pore fluid may be categorized in transient regime when

$$
\omega \tau_{\infty} \approx 1
$$

As $w r_{\text {eff }} \rightarrow 1$, the $\mathrm{F}^{-1}$ drizability of the system is then

$$
\alpha_{\omega}=\frac{3}{2} \epsilon_{m}\left(\frac{\epsilon_{g}-\epsilon_{m}}{\epsilon_{s}+2 \epsilon_{m}}+\frac{\sigma_{p}-\sigma_{m}}{\sigma_{p}+2 \sigma_{m}}\right)
$$

Figure 3.10 demonstrates the relationship between the pore fluid conductivity and $\omega \tau_{\text {eff }}$ for three common clay-water-electrolyte systems in Table 3.2. The effective relaxation time $r_{\text {eff }}$ is calculated from Eq.(3.26) and the ac angular frequency is

$$
\omega=2 \pi f=2 \pi(60 \mathrm{~Hz})=377 \mathrm{~Hz}
$$

It is shown in Fig.3.10 that at the low ac frequency, all the three common clay-waterelectrolyte systems are located in the transient regime and conductive regime, depending on the conductivity of pore water-electrolyte. It may be observed from Fig.3.10 that for the practical pore fluid conductivity values in natural clays, corresponding to the salinity of $0.005 \mathrm{~g} /$ or higher, the system is primarily located in the conductive regime, thus Eq.(3.32) may be used for the computation of effective polarizability for most cases.

The relative effective polarizability of three common clay-water-electrolyte systems in relation to the pore fluid conductivity can be illustrated in Fig.3.11, where 
the relative effective polarizability $\sigma_{\text {Keff }}$ is computed according to Eqs.(3.32) and (3.27) and plotted against the pore fluid conductivity $\sigma_{m}$ for the three common claywater-electrolyte systems described in Table 3.2. It may be seen that the effective polarizabilities in the three systems are basically identical in spite of their large differences in e apparent permittivities, which confirms that the clay systems are located in the conductive regime and Eq.(3.32) is a good approximation to the general equation (3.25) under the circumstances.

The characteristics of effective polarizability is clearly illustrated in Fig.3.11: (1). When the conductivity of pore fluid is lower than that of clay particles, typically $\sigma_{\mathrm{m}}<10^{-3} \mathrm{~S} / \mathrm{m}$ (Salinity $<0.005 \mathrm{~g} /$ )

the effective polarizar,ility is positive, i.e., the particles will be imposed a force towards the high electric field intensity and dielectrophoresis process will be positive. This represents the cases in which the salinity of pore fluid is very low.

(2). When the conductivity of pore fluid is higher than that of clay particles, tne effective polarizability is negative, i.e., the particles will be imposed a force directing away from the high field intensity and dielectrophoresis process will be negative. This is the case for most clay-water-electrolyte systems.

(3). When the conductivity of medium is similar to that of clay particles, typically in the range of $10^{-3} \mathrm{~S} / \mathrm{m}$ (salinity at about $0.005 \mathrm{~g} /$ ), the system is locat $\mathrm{d}$ in the critical state in terms of the action of dielectrophoresis. The direction of dielectrophoretic force could be towards either high or low field intensity, depending on the conductivity difference between the clay particles and pore fluid. The larger the 
the difference, the stronger the dielectrophoretic force would be on the clay particies. In the extreme case, when the pore fluid conductivity is identical to the clay mineral conductivity, the effective polarizability and the dielectrophoretic force would be zero and no particle motion could be observed in such a system.

The limitation of dielectrophoresis is revealed very clearly through the effective polarizability of clay-water-electrolyte systems. In the vicinity of the "critical conductivity", which is equal to the clay mineral conductivity in value, the magnitude of effective polarizability is nearly nil, implying that the dielectrophoresis in such a clay system is very limited even though a strong electric field is applied.

The principle demonstrated here is of particular importance with respect to applications of dielectrophoresis. Prior to any dielectrophoresis treatment, the conductivity of the pore fluid must be determined to evaluate the direction of dielectrophoretic force and the effectiveness of dielectrophoresis in the particular clay soil.

\subsubsection{Case 1: Effective Polarizability in Silica Colloidal System}

To examine the concept of effective polarizability, we go back to the aqueous silica discussed in Section 2.3.2 and Section 2.3.3.

If the relative permittivity and couductivity of a material is less than that of water and no double layer polarization takes place, a negative dielectrophoresis is expected, Eq. (2.18). In an experiment, therefore, one may observe the particles move to the region of lower field intensity, which is the case of the experiment described in Section 2.3.2. The size of the quartz sphere ( $1 \mathrm{~mm}$ ) was observed 
deflecting toward the low field intensity. Since the size of the sphere was much larger than that of colloids ( $1 \mathrm{~nm}-1 \mu \mathrm{m})$, the intrinsic permittivity of the quartz dominated the polarizability of the system. The influence of the electrical double layer is overshadowed by the volumetric properties as the specific surface area of the sphere is very small. ' the situation in colloid systems is different. Because of the small size and correspondingly large specific surface of colloidal particles, the electrical double layer contributes significantly to resulting forces. The polarization of the double layer dominates the dielectric property of the system. Upon imposing an electric field. the polarizability of a colloid system would depend on the effective polarizability of the suspension. The experiment described in Section 2.3 .3 has shown that the suspended silica particles moved into the high field intensity region, in contradictory to prediction of Eq.(2.18).

The silica-water system has been thoroughly investigated (e.g., Iler, 1970). A brief description is presented here with respect to the charge property at the silica-water-interface. In the interior of the silica colloidal particle, each silicon atom is normally covalently bonded in a tetrahedral fashion to four oxygen atoms. Each oxygen atom is in turn bonded to two silicon atoms, as shown schematically in Fig.3.12(a). The situation at the surface is different however, and this sequence must be interrupted. It can be pictured that each surface silicon atom lacks an oxygen atom to complete the $\mathrm{SiO}$, tetrahedron, and now each oxygen atom bonds only to one silicon atom. In the presence of water, the hydroxyl ion completes the tetrahedron of the surface silicon atom, while the hydrogen ion bonds to the nearby negatively charged surface oxygen atom of the surface, as illustrated in Fig.3.12(b). 
The overall effect is to give rise to surface charge. The charge density of silica cclloids as pH dependent. The isoelectric point, i.e. the point at which the particle surface exhibits zero net surface charge, for silica in water is estimated to occur at a pH of about 3.5 (Iler, 1970). The silica colloid surface is negatively charged at $\mathrm{pH}>\mathrm{pHo}=3.5$, which is the case in the colloidal silica systems reviewed in Section 2.3.3, where the $\mathrm{pH}$ value of the systems ranged from 4.4 to 8.0 , as seen in Fig.26(a).

The effective polarizability in an aqueous colloid system can be estimated in three steps, i.e, the Stern potential, the apparent permittivity and the effective polarizability. The apparent conductivity of silica gel is assumed as identical to that of quartz, as the volume fraction of particles in the test series is small ' $(p=0.1$, Scrimager, 1974).

- Stern potential of double layer

For colloidal silica at $\mathrm{pH}=8$, the surface charge density is determined to be in the order of $10^{12}$ electronic charge equivalents per $\mathrm{cm}^{2}$ (Bolt, 1957), i.e.

(1) $\rho=10^{12} \mathrm{e}_{0} / \mathrm{cm}^{2}=1.602 \times 10^{-3} \mathrm{C}^{2} \mathrm{~m}^{2}$

For the case of electrolyte $0.05 \times 10^{-3} \mathrm{~N} \mathrm{KNO}_{3}$

(2) $M=0.101 \mathrm{~kg}$

(3) $c=0.05 \mathrm{mN}=0.05 \mathrm{~mol} / \mathrm{m}^{3}$

(4) $T=293 \mathrm{~K}$

(5) $z=1$

The meaning of the symbols and the rest of parameters are the same as given in Section 3.3. 
Carrying out the calculation described in Appendix 2, we obtain the Stern potential of the colloidal silica as

$$
\Phi_{\mathrm{d}}=50.0 \mathrm{mV} \text {. }
$$

and the potential at particle surface

$$
\Phi_{0}=65.0 \mathrm{mV} \text {. }
$$

- Apparent permittivity of silica gel in aqueous solution

The zeta potential of the colloidal silica is assumed to be equal to the Stern potential. A typical particle fraction of 0.1 is assigned to the colloidal system. The relative permittivity of solution (water) is about 80 . Substitute these values and the ones obtained from previous step into Eq.(3.24) to calculate the relative apparent permittivity of silica colloids:

(8) $\quad K_{2}=223$

As seen the relative apparent permittivity of silica gel is in the order of $10^{2}$. Again the intrinsic relative permittivity of silica $\left(K_{p}=3.78\right)$ is completely overshadowed. - Effective polarizability of silica gel in aqueous solution

The frequency of the ac field was $1000 \mathrm{~Hz}$ as applied in the tests. Substituting the relative apparent permittivity $K_{a}=223$, the relative permittivity of medium (water) $K_{m}=80$, the conductivity of silica $\sigma_{\mathrm{p}}=10^{-18} \mathrm{~S} / \mathrm{m}$ and the conductivity of medium $\sigma_{\mathrm{m}}=10^{-6} \mathrm{~S} / \mathrm{m}$ (Scrimager, 1974) into Eq.(3.34) we obtain the effective relative polarizability

(9) $\quad \sigma_{\text {Keff }}=+87.8$

(10) $\omega r_{\text {eff }}=11$

The positive sign of the effective polarizability suggests that the particles will move 
to the region of higher field intensity in a non-uniform electric field, which was the case observed in the experiment. The term $\omega T_{\text {eff }}$ suggests that the system is located in the transient regime, in which the direction of dielectrophoretic force is very sensitive to the conductivity of the water electrolyte.

As seen in this case, the concept of effective polarizability in aqueous silica colloid systems was supported by the experimental evidences.

\subsubsection{Case 2: Effective Polarizability of Kaolinite-water-electrolyte System}

The dielectrophoresis experiments in clay-water-electrolyte systems with quantitative description were not reported in literature. However, the kaolinitewater-electrolyte systems reviewed in Section 3.3.2 may be used to estimate the possible dielectrophoretic response. The apparent permittivity and the apparent conductivity estimated according to the concept developed in Section 3.3.6 are consistent with the experimental results. We may reasonably postulate that the derived effective polarizability would provide some evidence of direction of particle motion and magnitude of dielectrophoretic force in a non-uniform electric field.

From calculation in Appendix 2, we have the follomedne for the kaolinitewater-electrolyte system:

(1) Apparent relative permittivity $K_{q}=282 \times 10^{4}$

(2) Relative permittivity of medium (water-electrolyte) $K_{m}=80$

(3) Conductivity of pore water-electrolyte $\sigma_{m}=0.153 \mathrm{~S} / \mathrm{m}$

(4) The clay particle conductivity $\sigma_{\mathrm{p}}=10^{-3} \mathrm{~S} / \mathrm{m}$ (Table 2.1)

Subatitute these values into Eqs.(3.25), (3.26) and (3.27) and assume an ac frequency 
of $60 \mathrm{~Hz}$ is applied to the system, we obtain

(5) $\quad w r_{\text {eff }}=\mathbf{0 . 0 0 5 6}$

(6) Relative effective polarizability of the system $\sigma_{\text {Ker }}=-118.8$

We found that the system is located in the typical conductive regime and the effective polarizability of the kaolinite system is negative, suggesting the clay particles tend to move toward the lower field intensity in a non-uniform electric field even under the effect of huge apparent permittivity due to double layer polarization. Although the experimental evidence to the prediction in the kaolinite system is not available, there have been experimental indications that clay particles tend to move away from high electric field region observed indirectly from movement of water in the clay systems ( Lockhart, 1983; Lo et al, 1992) as reviewed in Chapter 2.

\subsubsection{Summary}

In this section we analyzed the "effective polarizability" in aqueous colloid systems with emphasis on clay-water-electrolyte systems. The effective polarizability, which is the goveming factor of the direction of dielectrophoretic force imposed on suspended particles, is primarily controlled by the conductivity of aqueous medium.

For most clay-water-electrolyte systems with pore water conductivity of $10^{-3}$ S/m (salinity $0.005 \mathrm{~g} /$ ) or higher value, the following conclusions may be drawn: 1. The effective polarizability is negative, indicating that the clay particles will experience a dielectrophoretic force outward from the central electrode in a nonuniform electric field.

2. The magnitude of effective polarizability is not affected by the clay mineral 
type, implying a consistent action of dielectrophoretic force upon clay particles.

3. There is a "critical" pore fluid salinity at about $0.005 \mathrm{~g} /$, which indicates that dielectrophoresis would be less or not effective if the pore fluid has the equivalent electrolyte concentration.

\subsection{Conclusions}

The analysis conducted in this chapter is of fundamental importance in order to understand the dielectrophoretic action in clays. The following conclusions may be drawn with respect to the dielectrophoretic force in clay-water-electrolyte systems: 1. The intensity and gradient of the non-uniform electric field are the control factors of the magnitude of dielectrophoretic force. The distribution of the electric field intensity and gradient may be represented by equi-intensity lines and was analyzed for several practical non-uniform electric field configurations with ideal or approximate cylindrical symmetry.

2 The electrical double layer polarization in aqueous colloid systems will induce abnormally high permittivity in an electric field. The conduction behaviour of claywater-slectrolyte systems is dominated by the conductivity of pore water-electrolyte.

3. An "effective polarizability" is introduced. The effective polarizability is proportional to the magnitude of the dielectrophoretic force. The sign of the effective polarizability controls the direction of dielectrophoretic force, i.e., either towards or away from the central electrode.

4. In most clay-water-electrolyte systems, the effective polarizability is negative, indicating that the clay particles will experience a dielectrophoretic force polnting 
outward from the central electrode.

5. The magnitude of effective polarizability in clay-water-electrolyte systems is dependent on the conductivity of soil water and not appreciably affected by the clay mineral type. 
TABLE 3.1 DIELECTRIC PROPERTIES OF KAOLINITE-WATERELECTROLYTE SYSTEM (after Arulanandan and Mitchell, 1968)

3.1a Effect of Temperature

\begin{tabular}{llll}
\hline Soil Type & Temperature $^{\circ} \mathrm{F}$ & $\mathrm{K}=\epsilon / \epsilon_{\mathrm{o}} \times 10^{4}$ & $\sigma(\mathrm{mS} / \mathrm{m})$ \\
\hline Sodium Kaolinite & 55.0 & 7.0 & 36.6 \\
$(2 \mu \mathrm{m})$ & 72.5 & 8.0 & 48.0 \\
& 90.0 & 9.0 & 60.0 \\
& 105.0 & 9.5 & 71.0 \\
\hline
\end{tabular}

Note: Pore fluid $=0.01 \mathrm{~N} ;$ Water content of sample $=64 \%$

3.1b Effect of Particle Size

\begin{tabular}{llll}
\hline Grain Size & Soil Type & $K=\epsilon / \epsilon_{0} \times 10^{4}$ & $\sigma(\mathrm{mS} / \mathrm{m})$ \\
\hline Fine $(\sim 3 \mu \mathrm{m})$ & Kaolinite ASP900 & 21.5 & 94 \\
Coarse $(\sim 7 \mu \mathrm{m})$ & Kaolinite Huber 45 & 53.5 & 94 \\
\hline
\end{tabular}

Note: Temperature $=69-70^{\circ} \mathrm{F} ;$ Water content $=75 \% ;$ Pore fluid $=$ distilked water

3.1c Effect of Electrolyte Concentration

\begin{tabular}{lllll}
\hline Soil Type & $\begin{array}{l}\text { Electrolyte } \\
\text { Concentration }\end{array}$ & $K=\epsilon / \epsilon_{\mathrm{o}} \times 10^{4}$ & $\sigma(\mathrm{mS} / \mathrm{m})$ & $\begin{array}{l}\sigma_{\mathrm{m}}(\text { pore } \\
\text { fluid) } \\
(\mathrm{mS} / \mathrm{m})\end{array}$ \\
\hline Kaolinite & $0.002 \mathrm{~N}$ & 1.8 & 21.2 & 25 \\
Hydrite 'R' & $0.01 \mathrm{~N}$ & 2.7 & 51.4 & 153 \\
$(1 \mu \mathrm{m})$ & $0.05 \mathrm{~N}$ & 2.9 & 128.4 & 448 \\
\hline
\end{tabular}

Note: Temperature $=69-70^{\circ} \mathrm{F} ;$ Pore fluid $=\mathrm{NaCl} ;$ Water content $=47.5 \%$ 
81

(continued)

TABLE 3.1 DIELECTRIC PROPERTIES OF KAOLINITE-WATERELECTROLYTE SYSTEM (after Arulanandan and Mitchell, 1968)

3.1d Effect of Cation Type

\begin{tabular}{lllll}
\hline Soil Type & Pore fluid & $\begin{array}{l}\mathrm{K}=\epsilon / \epsilon_{0} \times \\
10^{4}\end{array}$ & $\begin{array}{l}\sigma \\
(\mathrm{mS} / \mathrm{m})\end{array}$ & $\begin{array}{l}\sigma_{\mathrm{m}} \text { (Pore } \\
\text { fluid) }(\mathrm{mS} / \mathrm{m})\end{array}$ \\
\hline Kaolinite & $\mathrm{LiCl}$ & 3.7 & 38.4 & 83 \\
Hydrate 'R' & $\mathrm{NaCl}$ & 2.7 & 51.4 & 153 \\
$(1 \mu \mathrm{m})$ & $\mathrm{KCl}$ & 2.9 & 128.4 & 448 \\
\hline
\end{tabular}

Note: Temperature: $69-70^{\circ} \mathrm{F}$; Water content: $47.5 \%$

Notations:

K: Apparent relative permittivity.

$\sigma$ Conductivity of clay-water-electrolyte system.

$\sigma_{\mathbf{m}}:$ Conductivity of pore fluid. 


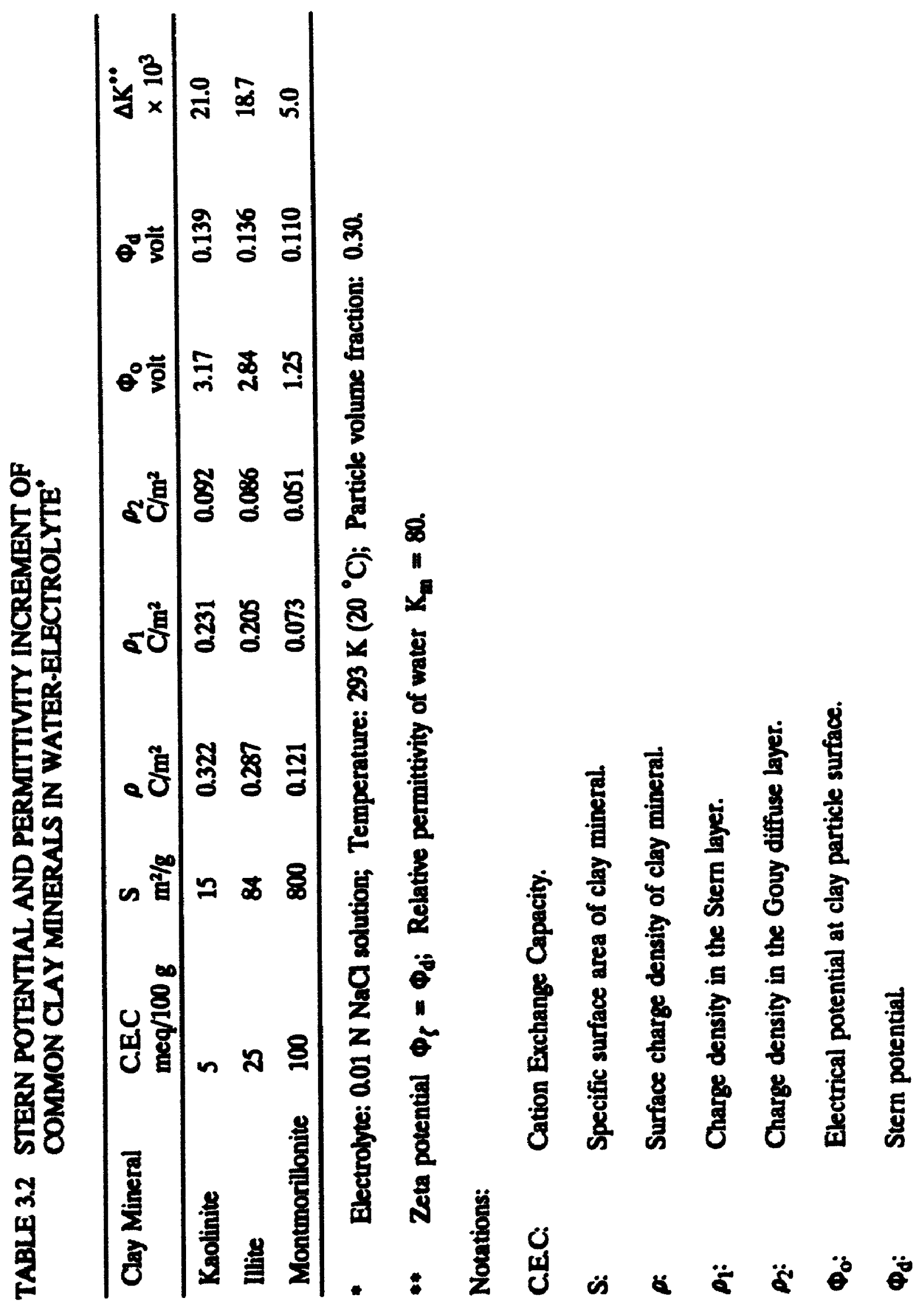




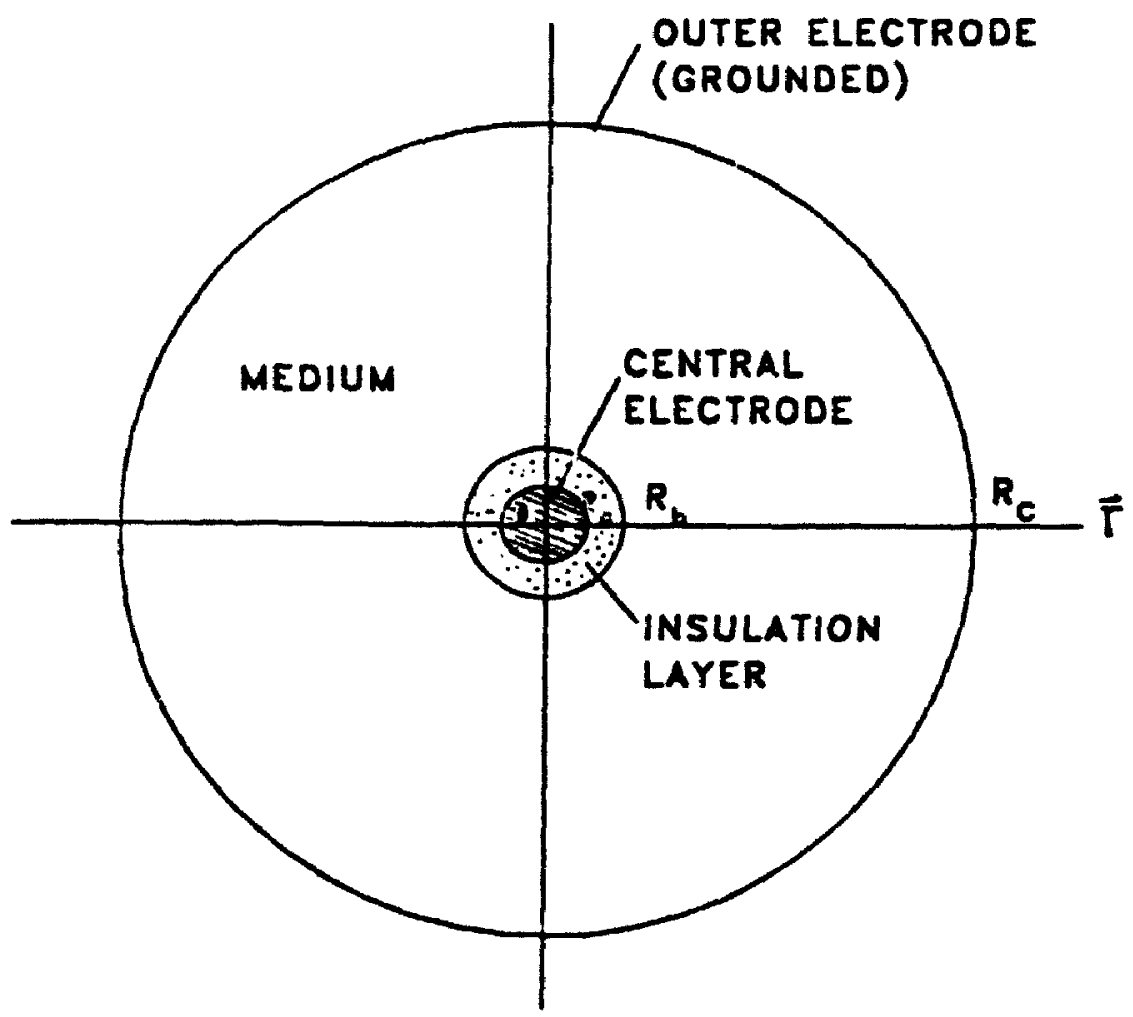

FIGURE 3.1 CYLINDRICAL ELECTRIC FIELD GEOMETRY (TOP VIEW) 


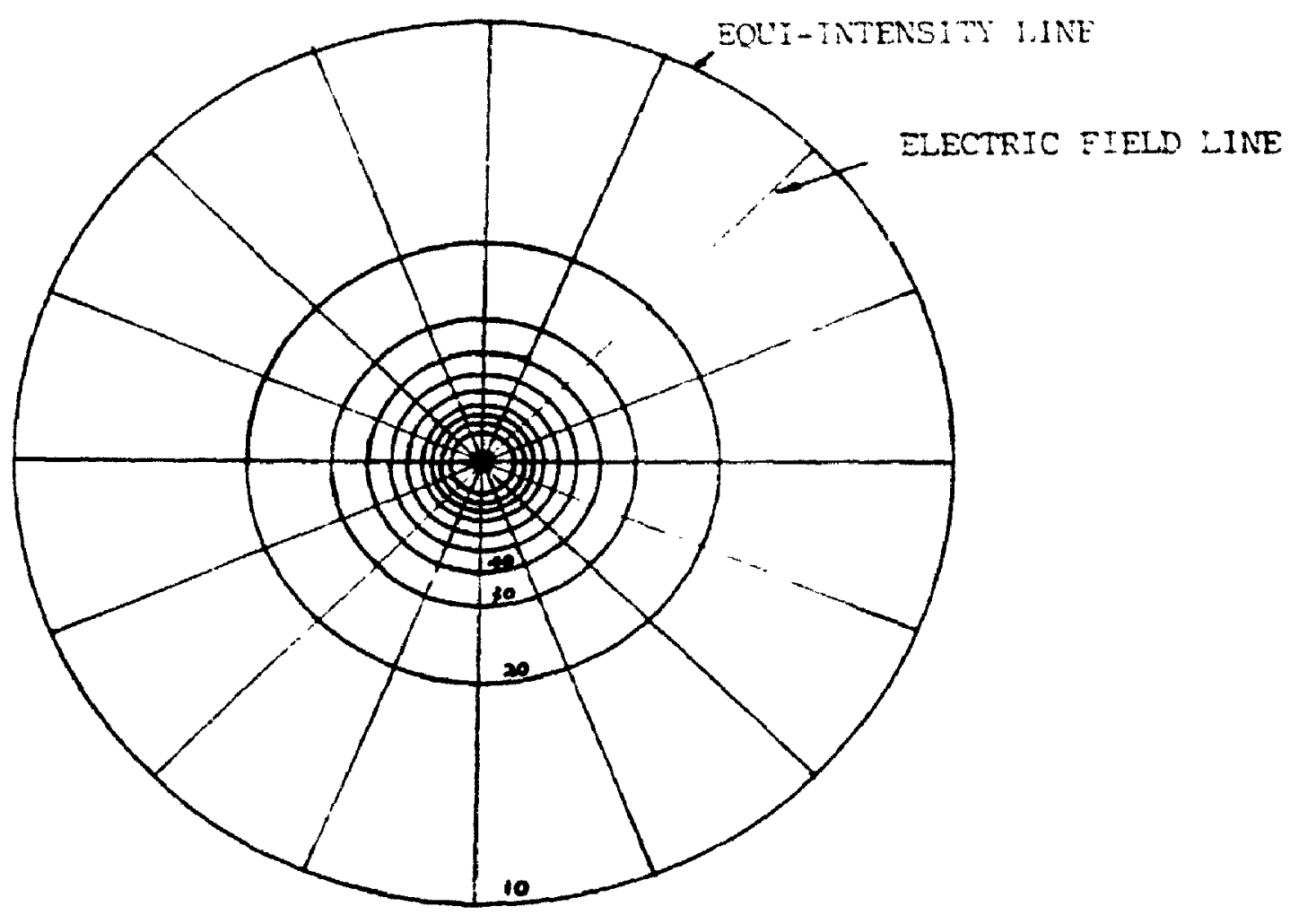

FIGURE 3.2 EQUI-INTENSITY LINES AND ELECTRIC FIELD LINES IN A CYLINDRICAL FIELD 


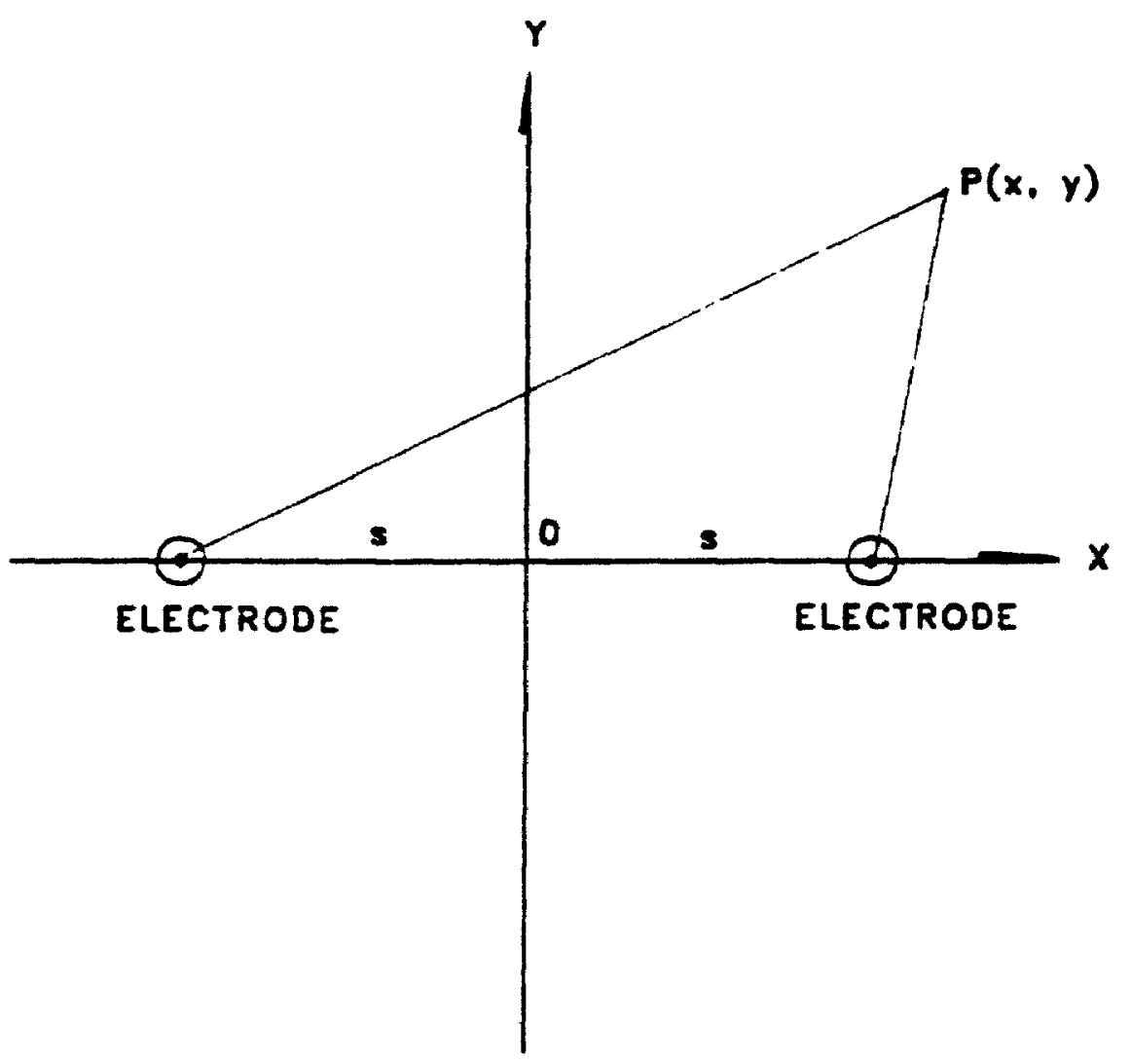

FIGURE 3.3 ELECTRODE-PAIR: PLAN VIEW. LENGTH OF ELECTRODE: $L$ 

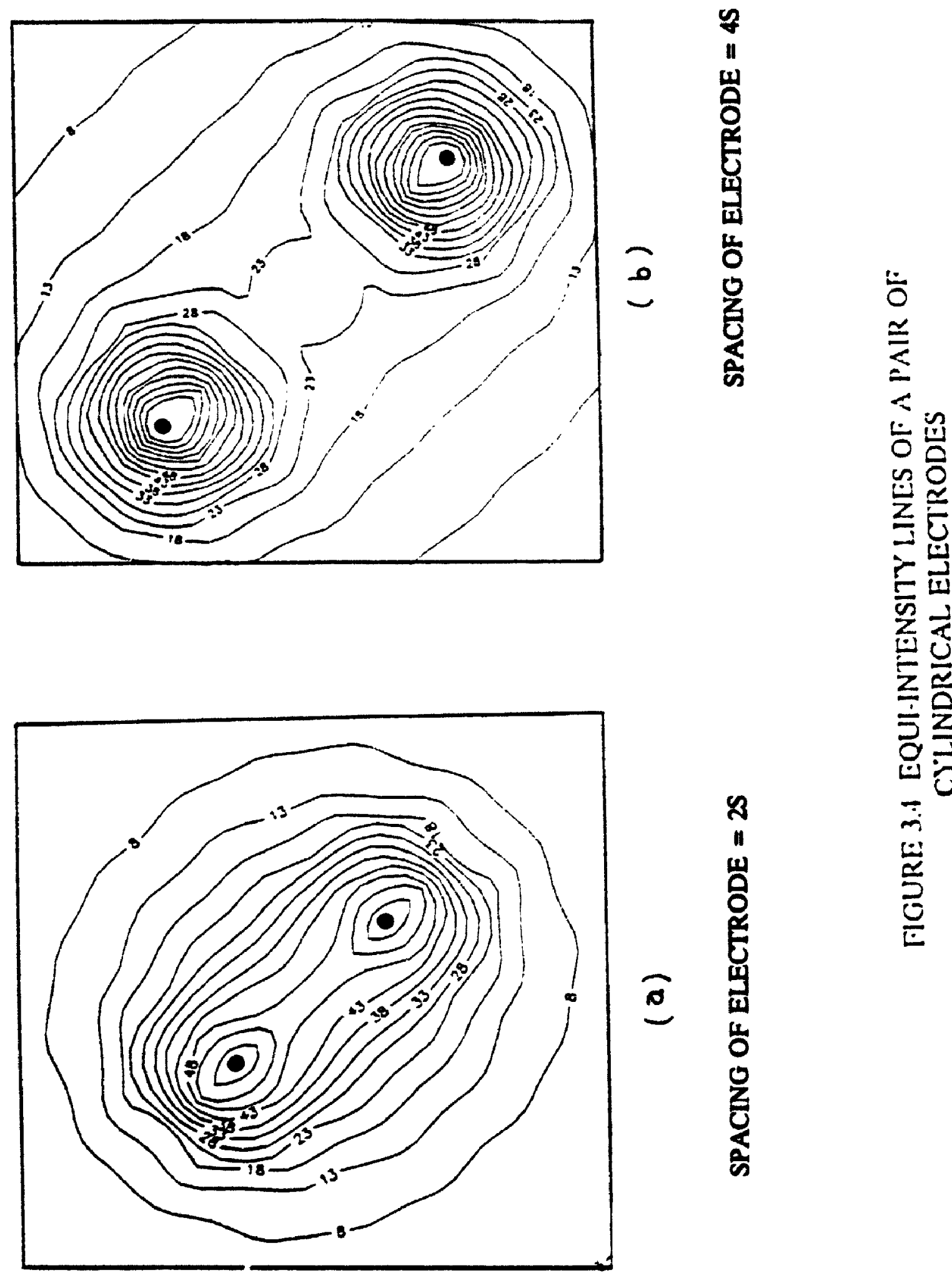


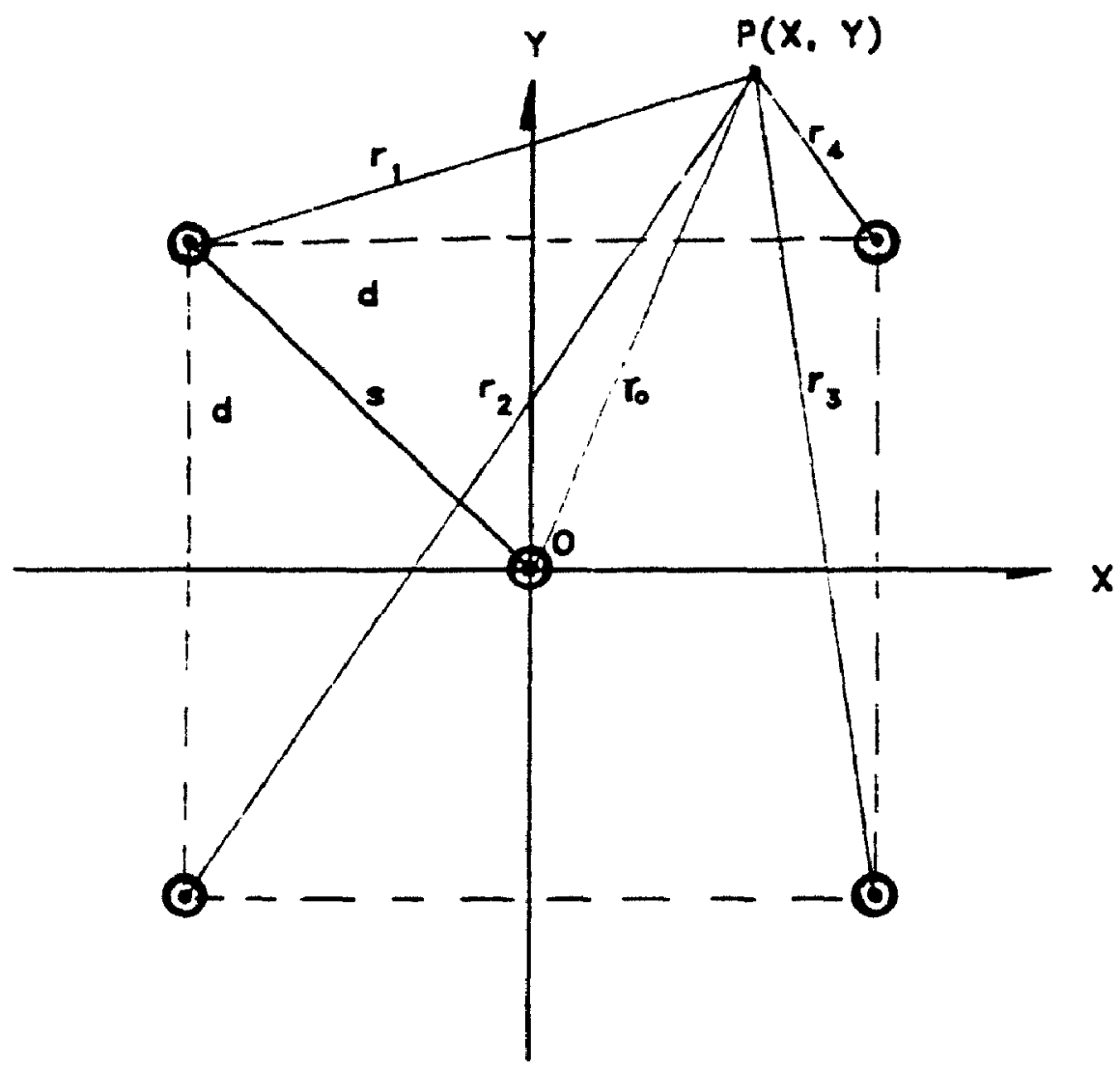

O electrode

FIGURE 3.5 ELECTRODE-MATRIX (PLAN VIEW) LENGTH OF ELECTRODE: L 


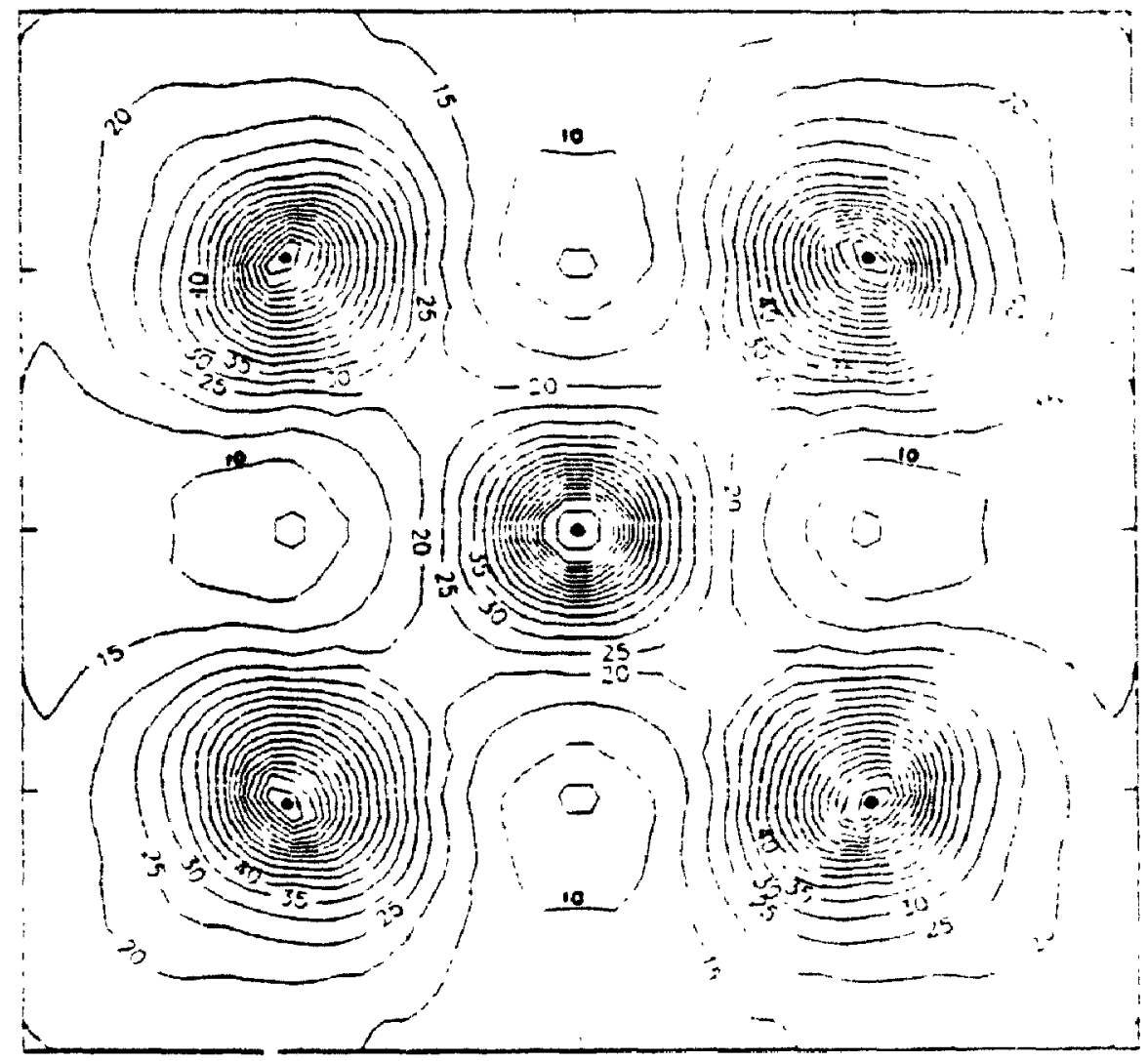


STERN LAYER

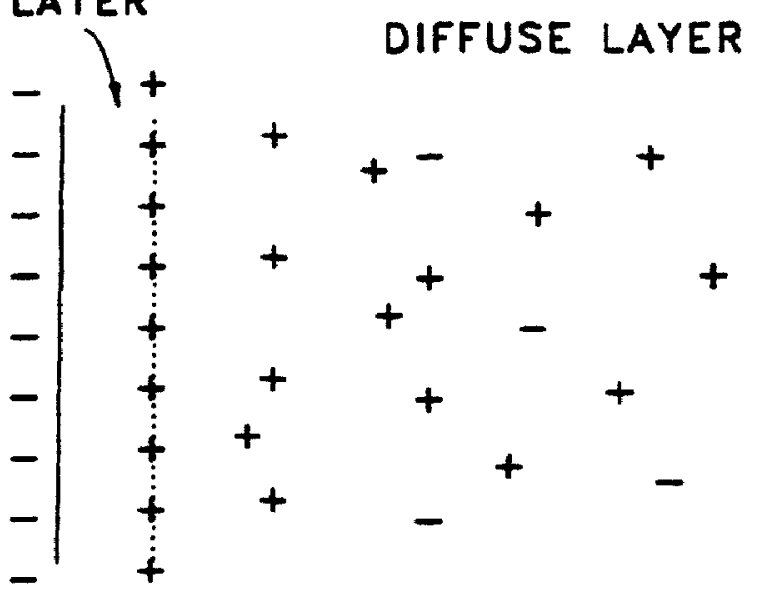

(A) CHARGE DISTRIBUTION AS RELATED TO DISTANCE

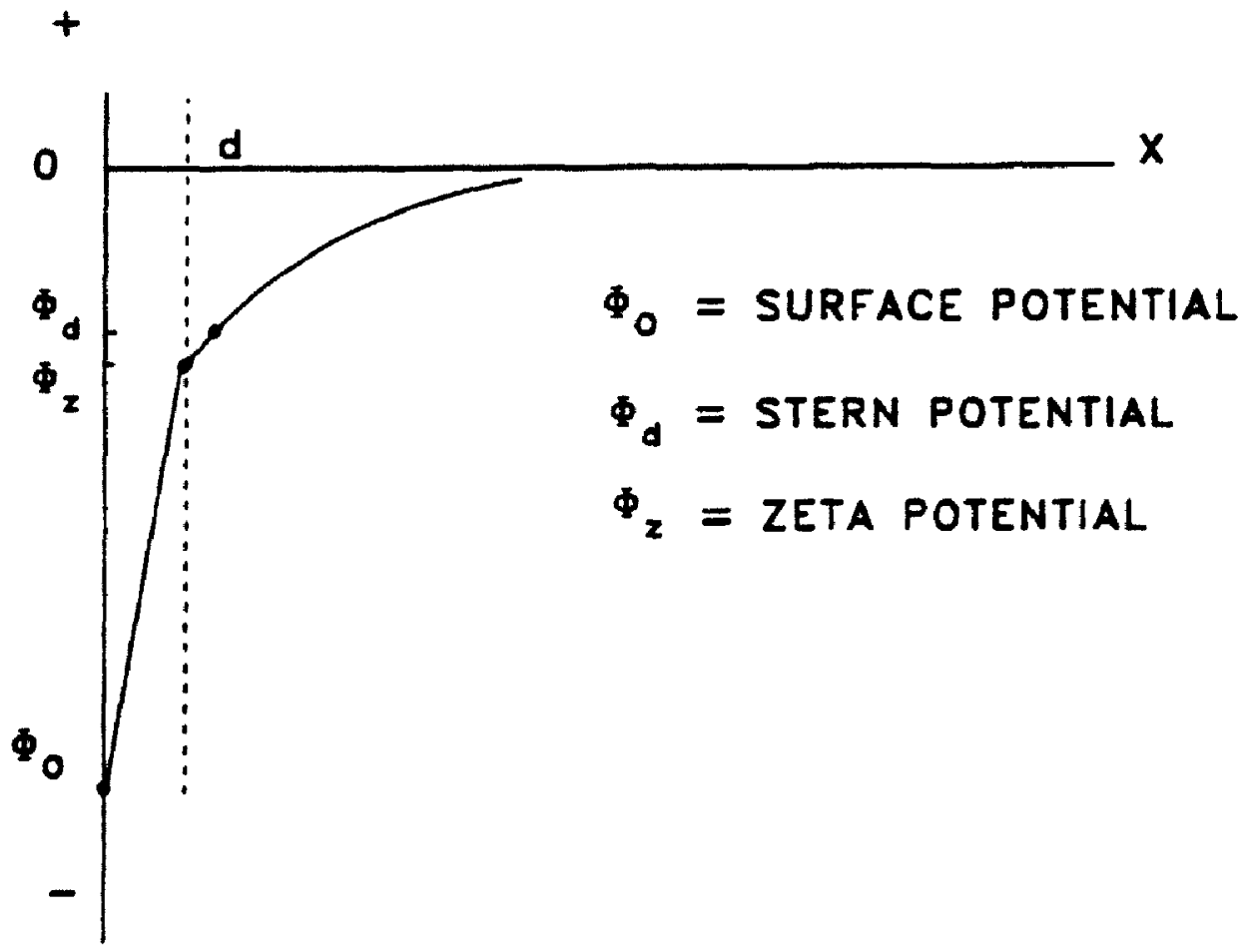

(B) POTENTIAL DISTRIBUTION AS RELATED TO DISTANCE

FIGURE 3.7 ELECTRICAL DOUBLE LAYER: GOUY-STERN MODEL

( AFTER SPARKS, 1986) 


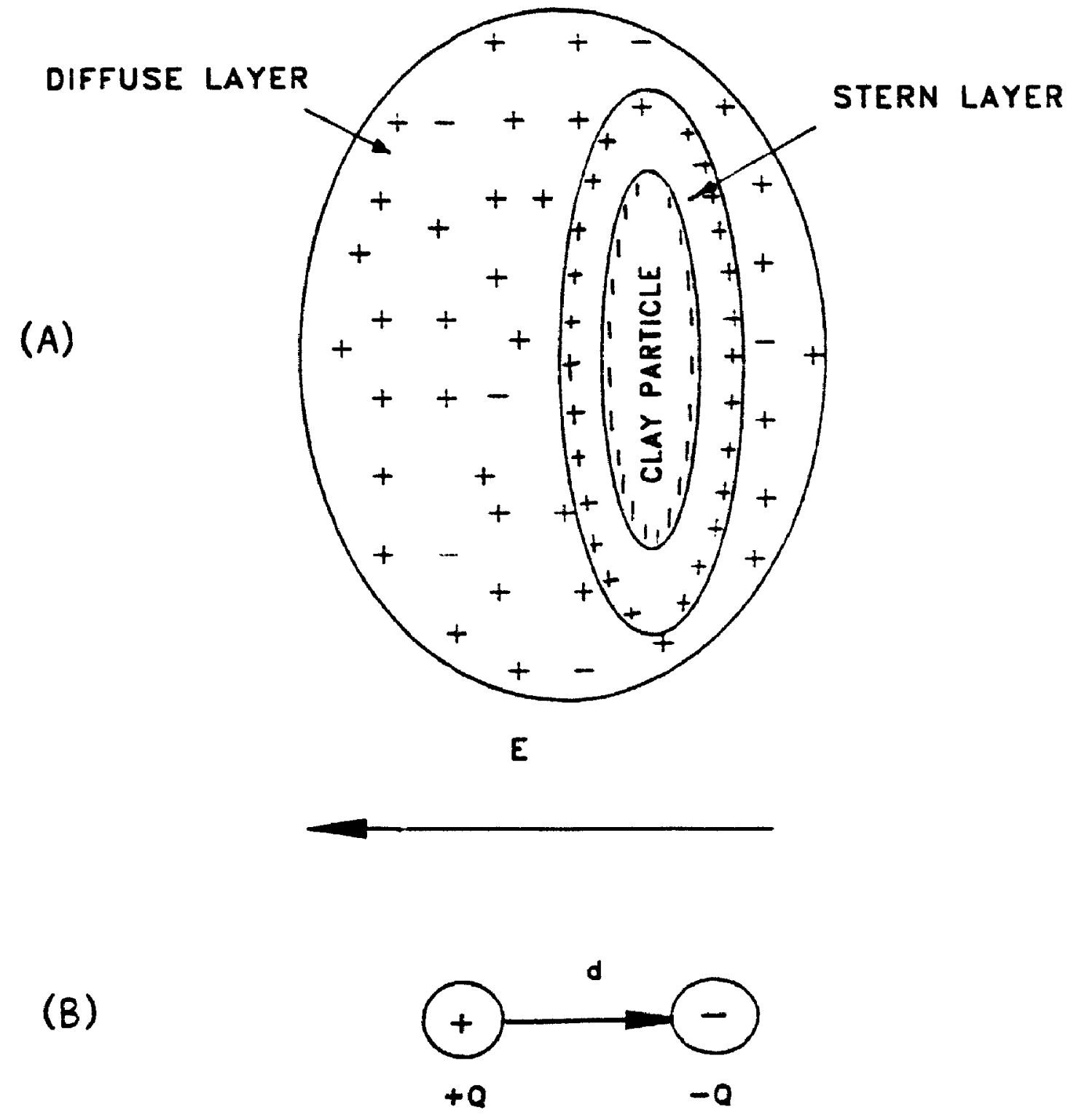

FIGURE 3.8 ILLUSTRATION OF DOUBLE LAYER POLARIZATION

(A) POLARIZATION OF DIFFUSE LAYER IN AN

(B) EOUTVALENT DIPOLE

(AFTER POHL, 1978, REVISED) 

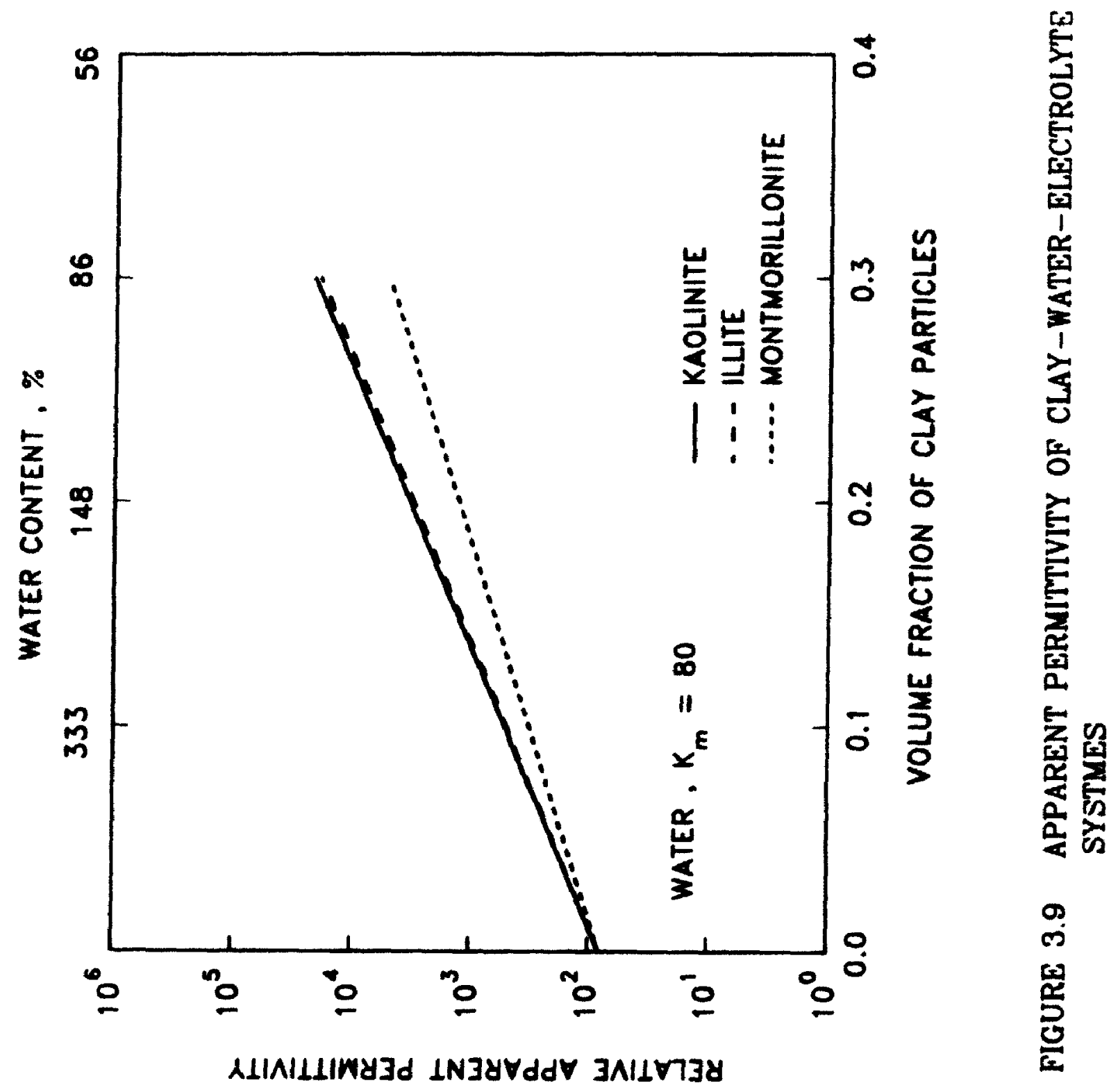


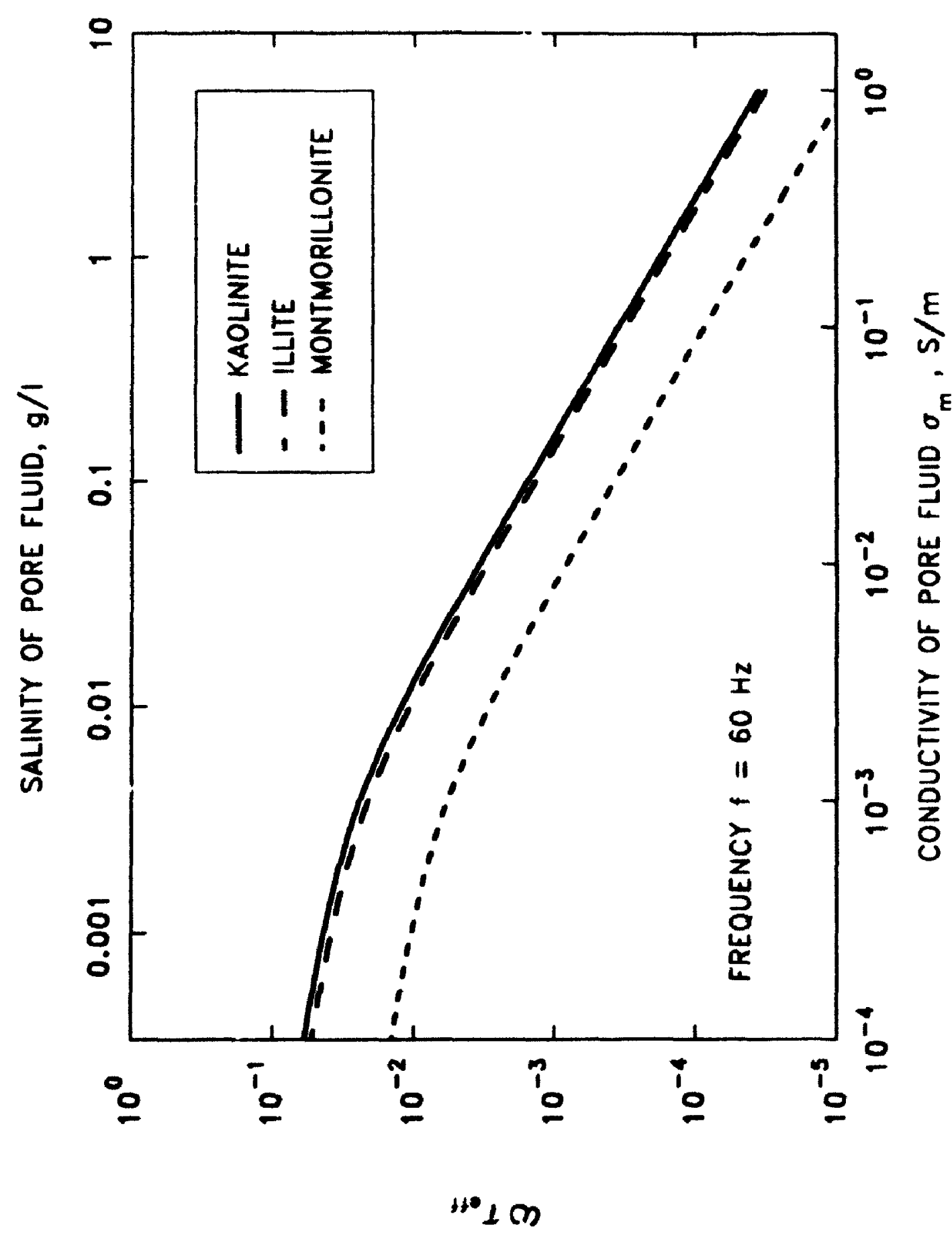

资 


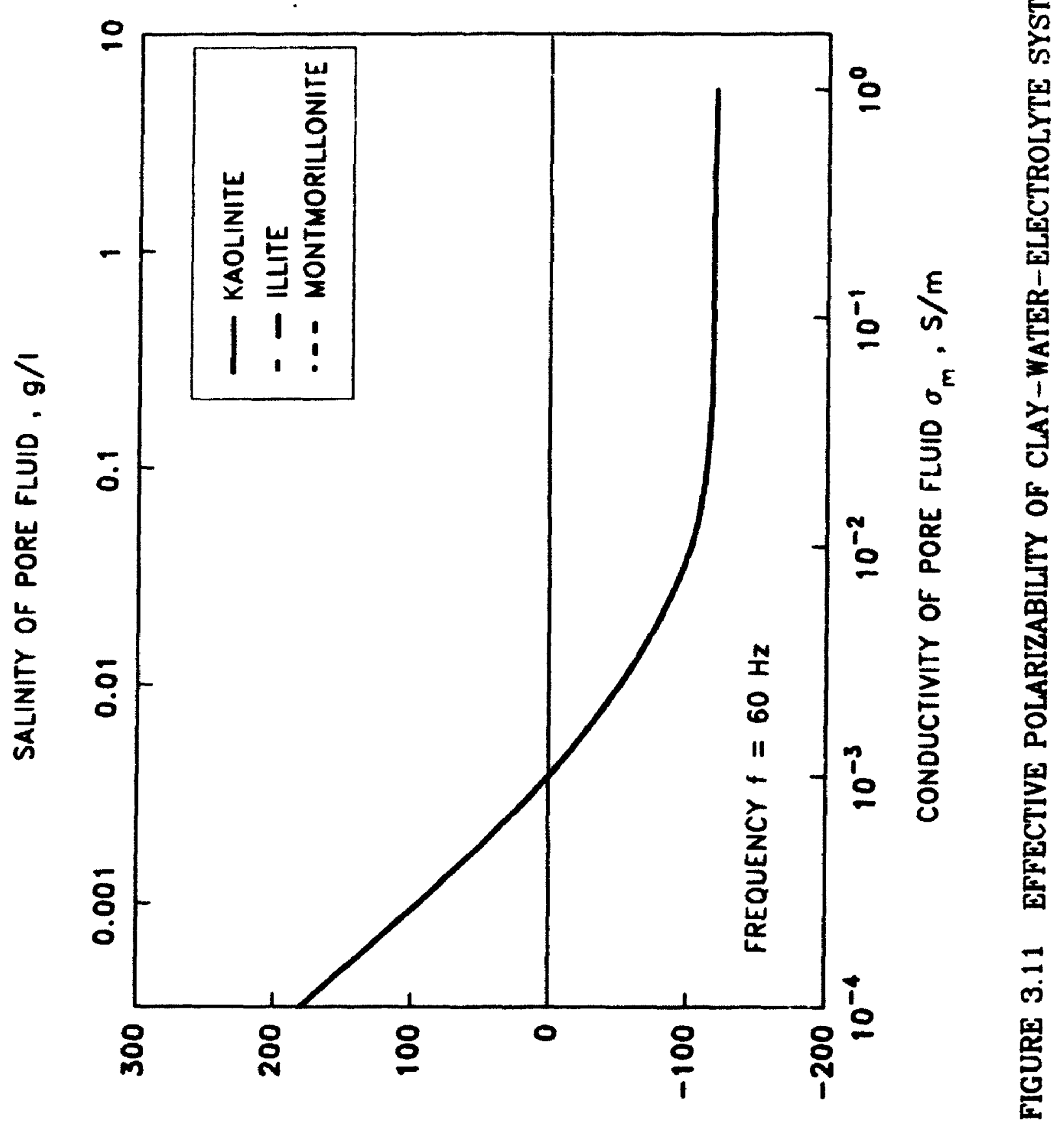

"מ०x 
Suspensions and colloids
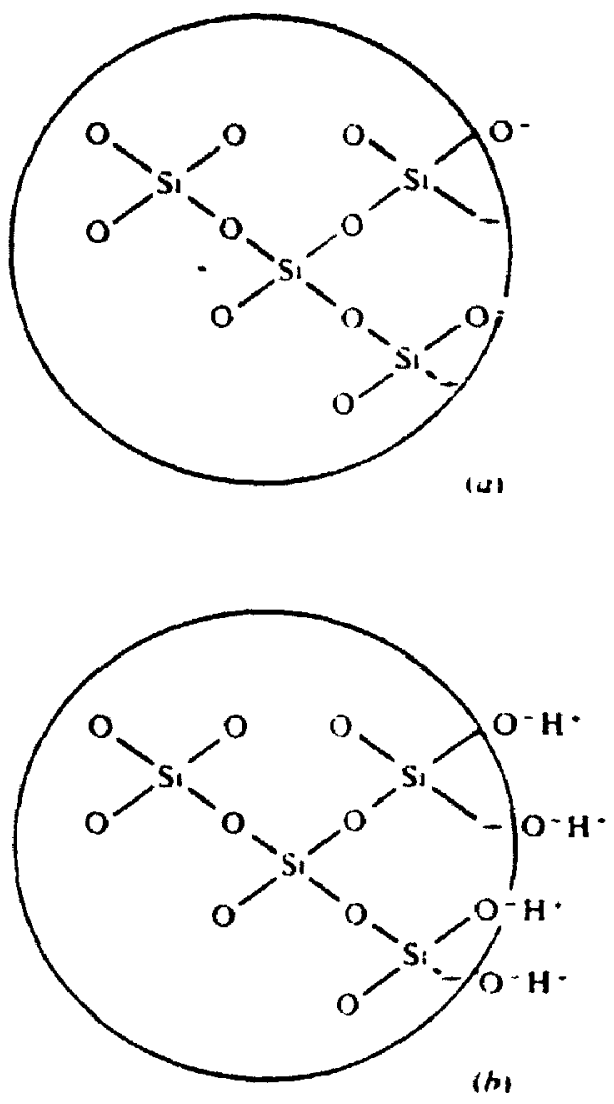

FIGURE 3.12 SILICA PARTICLE IN WATER (AFTER POHL, :978) 


\section{CHAPTER 4}

\section{GEOTECHNICAL AND DIELECTRIC PROPERTIES OF TWO LEDA CLAYS}

\subsection{Introduction}

Leda clay ( Champlain Sea clay) is the soil used in this study for investigation of dielectrophoresis. The clay samples were recovered in two sites. One is located at Gloucester, Ontario, about $20 \mathrm{~km}$ south-east of Ottawa city and the other is at Orleans, Ontario, about $20 \mathrm{~km}$ east of Ottawa city. The distance between the two sites is about $10 \mathrm{~km}$.

The sedimentary deposit encompassed the area is the marine deposit of Champlain Sea clay which covers the entire St. Lawrence Lowland and Ottawa valley. The geological history of the deposit had been extensively investigated (Karrow, 1961; Crawford, 1961; Quigley, 1980 ). Basically, the Champlain Sea occupied the St. Lawrence Lowlands from approximately 12,500 to $10,000 \mathrm{BP}$. The sea is believed to have invaded the lowlands from the Gulf of St. lawrence when the ice dam melted in the vicinity of Quebec City. It mixed with fresh water lakes in the valley and marine clays were subsequently deposited over freshwater varved clays in many areas. The sediment was formed through various stages of deposition, erosion and re-deposition. It has been reported that the normal composition of clay from the Champlain Sea is one dominated by shield-derived primary minerals such as quartz, feldspar, amphibole, illite and chlorite (Quigley, 1980).

The geotechnical and dielectric properties of the Gloucester clay and Orleans 
clay are reported in this chapter as determined in a series experimental studies.

\subsection{Geotechnical Properties}

\subsubsection{Gloucester Clay}

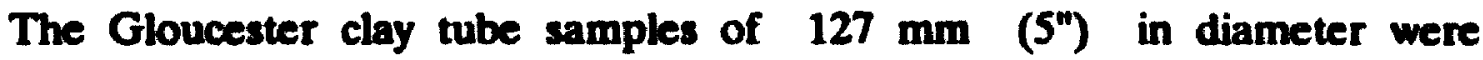
recovered from the Gloucester Test embankment site of the National Research Council of Canada, at the depth between $2 \mathrm{~m}$ to $6 \mathrm{~m}$.

(1) Stratigraphy and soil properties

The subsoil condition at the Gloucester test site consists of $20 \mathrm{~m}$ Leda clay deposit, which is classified into three basic layers according to index properties, undrained shear strength and pre-consolidation pressure (Bozozuk and Leonards, 1972 ; Lo et al, 1976). Layer 1 compriaing of $2.3 \mathrm{~m}$ desiccated top crust overlies $4.7 \mathrm{~m}$ soft grey silty clay. Layer 2 is a $6.1 \mathrm{~m}$ thick grey clay and layer 3 is a $5.1 \mathrm{~m}$ thick grey silty clay. Summarized soil conditions are shown on the borehole log Fig.4.1. Tube samples of $127 \mathrm{~mm}$ nominal diameter were obtained from the soft grey silty clay stratum of layer 1 . The soil properties are summarized in Table 4.1.

The Gloucester clay is a typical soft senaitive clay with very low shear strength. The sensitivity of the clay is as high as 100 at wome locations. The in-situ water content is well above the liquid limit, indicating the highly sensitive nature of the soil. The high values of brittleness index and undrained modulus are the characteristics of the Gloucester clay, as shown in Table 4.1. The soil is basically a slightly over-coneolidated clay with an over-consolidation ratio of 1.5 and a fairly low pre-consolidation presure of 35 to $87 \mathrm{kPa}$. 
(2) Chemical analysis

The results of chemical inalysis are presented in Table 4.2 The soluble salt content of the pore water is $1.26 \mathrm{~g} /$, consistent with previous report in this area (Crawford, 1961; Soderman and Quigley, 1965). The percentage of $\mathrm{K}_{2} \mathrm{O}$ found in the clay sample can be attributed to illite as no other potassium bearing minerals were identified on the X-ray traces. Using a value of $3.30 \% \quad \mathrm{~K}_{2} \mathrm{O}$, the clay is computed to contain about $55 \%$ illite.

(3) Mineralogical analysis

A clay sanıple from the depth of $6 \mathrm{~m}$ was retained for mineralogical analysis. The powder patten of the X-ray trace is shown in Fig.4.2 while the other traces are enclosed in Appendix 4. It is shown that the Gloucester clay contains abundant illitemica, chlorite, quartz and feldspar with a small but significant amount of amphibole.

The abundant illite is present as evidenced by the strong reflection at $1.004 \mathrm{~nm}$ on all traces. The peaks at $1.4 \mathrm{~nm}$ and $0.7 \mathrm{~nm}$ are iron chlorite peaks. Heat treatment at $550^{\circ} \mathrm{C}$ for 30 minutes caused the $0.708 \mathrm{~nm}$ peak nearly to disappear and the $1.402 \mathrm{~nm}$ peak to intensify, confirming the presence of chlorite, probably the high iron variety (Soderman and Quigley, 1965). Glycolation of potassium clay and natural clay did not cause shift of the peaks, which negated the existence of montmorillonite-like swelling minerals.

Non-clay minerals identified from the powder patterns of the clay size fractions indicate the presence of abundant feldspar, quartz and trace of amphibole. The composition of the Gloucester clay is summarized in Table 4.3. The values are close to those given by Soderman and Quigley (1965). 


\subsubsection{Orleans Clay}

The Orleans clay block samples were recovered from a site about $\mathbf{2} \mathbf{k m}$ east of Orleans township at the proposed interchange of Regional Road 47 and Highway 17.

(1) Soil stratigraphy and field sampling

The site is flat with wild vegetation coverage. The surface drainage is towards the north. A layer of desiccated silty stiff clay crust underlies the top soil. The Leda clay starts at about $4 \mathrm{~m}$ below the ground level. The thickness of the Leda clay layer is about $6 \mathrm{~m}$ to $7 \mathrm{~m}$, overlaying on the limestone bedrock. The ground water table is at about $3.3 \mathrm{~m}$ to $3.7 \mathrm{~m}$ below the ground level. The borehole log showing the soil stratigraphy is presented in Fig.4.3.

The excavation for the block sampling was carried out by a track-mounted excavator. The excavation was about $400 \mathrm{~m}^{2}$ in plan area, $5.2 \mathrm{~m}$ in depth with a working area of $4.5 \mathrm{~m}$ by $4.5 \mathrm{~m}$ at the bottom. Fig.4.4 shows the general view of the excavation.

The Leda clay in the sampling area is dark grey in colour, silty, homogeneous with occasional cobble inclusions. Comparing to the Gloucester clay, the Orleans clay is much stiffer at undisturbed condition. Upon disturbance, however, it becomes very soft and slippery, showing fairly high sensitivity.

The block samples, $300 \mathrm{~mm}$ cube in dimensions, were cut off from the platform by a grooving tool specially designed for block sampling of soft clays, as shown in Fig.4.5. At the first the two vertical sides of a block were grooved into a $10 \mathrm{~mm}$ channel down to the desired depth. Then a steel-wire-cutter was threaded 
into the channel to cut the block off. The soil blocks. each about $50 \mathrm{~kg}$ in weight, were carried out of the pit and waxed immediately with double cheese cloth reinforcement. The block samples were carefully stored in individually padded boxes and transported back to the Geotechnical Research Laboratory of the University of Western Ontario.

(2) Soil properties

The laboratory testir.g of the Orleans clay was conducted immediately after sampling. The results of soil properties are summarized in Table 4.1. The Orleans clay has broadly similar water content, specific gravity and unit weight as those of the Gloucester clay. However, certain differences can be observed from the other properties. The Orleans clay is a stiff clay, with the undrained shear strength of $61.7 \mathrm{k} .1$ to $68.8 \mathrm{kPa}$ and pre-consolidation pressure of $142 \mathrm{kPa}$ to $146 \mathrm{kPa}$, much higher than those of the Gloucester clay. The brittle.ess of the Orleans clay is noticeably higher that the Gloucester clay, as indicated by the brittleness indexes and undrained moduli. The other distinct difference between the two clay soils is the higher over-consolidation ratio of the Orleans clay. The Orleans clay is an overconsolidated clay with the overconsolidation ratio of 2.4 . The Orleans clay is less sensitive than that of the Gloucester clay in terms of the liquidity index and sensitivity. By these measures, the properties of the Orleans clay and the Gloucester clay represent the upper and lower limits of the Leda clay in this area (Crawford, 1961).

(3) Soil composition

The chemical analysis results to the Orleans clay is summarized in Table 4.2. 
It can be seen that the chemical properties of the two soils are rather comparable. characterized as those of the Leda clay. The percentage of illite is determined as $58 \%$ according to the percentage of $K_{2} O(3.48 \%)$, nearly identical to that in the Gloucester clay based on same assumption in Section 4.2.1. Among noticeable differences are the slightly lower carbonate content, salinity (pore fluid conductivity) and C.E.C. value in the Orleans clay than those in the Gloucester clay. Considering the almost identical illite contents in the two soils, the lower C.E.C. value in the Orleans clay should be attributed to the lower chlorite content which is estimated as about $4 \%$ according to the C.E.C. value.

The powder pattern of the X-ray trace for the Orleans clay is presented in Fig.4.6 and the other traces are enclosed in Appendix 4. It can been seen from the powder pattern that $1.4 \mathrm{~nm}$ peak of the characteristic reflection of chlorite is almost unidentifiable and the $0.713 \mathrm{~nm}$ peak disappeared. The reflection intensity analysis gives an estimated chlorite content of $9 \%$, comparing to about $13 \%$ in the Gloucester clay. Taking the results from C.E.C. and X-ray data into account, the chlorite content in the Orleans clay is estimated as $5-10 \%$.

The analysis of the other X-ray traces are similar to that for the Gloucester clay.

The composition of the Orleans clay based on combined analysis to the chemical and X-ray data is summarized in Table 4.3.

\subsubsection{Summary}

The clay soils to be used in this study are typical sensitive clays of the Leda 
clay origin. The soils are characterized by the very low remoulded shear strength (high sensitivity), high in-situ water content close to or greater than the liquid limit (high liquidity index). These behaviours is attributed to an open, flocculated structure. The clays are highly brittle with the typical failure strain less than $1 \%$. The mineral composition of the Leda clays is an abundance of illite-mica, varying amount of chlorite, significant quartz and feldspar and small amount of amphibole.

\subsection{Dielectric Properties}

The dielectric properties of clay-water-electrolyte systems are of particular importance for studying dielectrophoresis process. The problem may be approached in two ways. In the first way, synthetic soils are used. Soils of known composition are prepared by blending different commercially available clay minerals with each other. The pore fluid ion concentration and type may also be controlled by pretreatment to the soil samples. The experimental study of Arulanandan and Mitchell discussed in Chapter 3, Section 3.3.2 is an example. This approach has certain advantages in terms of problem specification. However, it has the disadvantages that the dielectric properties of the pure minerals may not necessarily be the same as those of the minerals in the natural soil and the influence of soil structure is not taken into account.

In the second approach, natural soils are used. The dielectric properties are determined together with the composition and engineering properties, and correlations are made. The approach has the advantage that measured properties are those of soil in nature, so that the effect of soil structure can be investigated. 
An experimental technique is developed in this study to determine the dielectric properties of natural soils. The co-relations between the soil water content (volume fraction of clay particles) and dielectric properties, i.e., permittivity and conductivity, are investigated under both undisturbed and remoulded soil conditions. The effective polarizabilities of two Leda clays described in Section 4.2 are estimated according to the experimental data. The data may be used to estimate the direction of dielectrophoretic force imposed on clay particles.

\subsubsection{Experimental Technique}

(1) Principle

The permittivity and conductivity of a material can be determined by measuring the impedances of the material in a fixed test cell acting as an electrical capacitor-resistor. It is known that for an ideal parallel plate capacitor or a rectangular shaped resistor, the capacitance $C$ and conductance $G$ can be calculated respectively from

$$
\begin{array}{ll}
C=\frac{e A}{t}, & \text { farad } \\
G=\frac{\sigma A}{t}, & \text { siemens }
\end{array}
$$

where

6 : permittivity of the material between the plates, $\mathrm{F} / \mathrm{m}$;

A : section area of the plates, $\mathbf{m}^{2}$;

t: separation distance between the plates, $\mathrm{m}$; 
$\sigma$ : conductivity of the material between the plates, $S / \mathrm{m}$.

Note that the section area of the plates must be much larger than the separation distance so that the boundary effect can be neglected.

In principle, therefore, the permittivity and conductivity of a material can be determined by inserting the material into the space of two parallel conducting plates and measuring the corresponding capacitance $\mathbf{C}$ and conductance $\mathbf{G}$ as the section area and separation distance of the plates can be pre-determined. From Eq.(4.1) we have

$$
\begin{aligned}
& K=\frac{e}{e_{0}}=\frac{C t}{A e_{0}} \\
& \sigma=\frac{G t}{A}
\end{aligned}
$$

where $K$ is the relative permittivity, $\epsilon_{0}$ is the permittivity of vacuum and the other symbols are as defined before. Note that the conductance $\mathbf{G}$ and resistance $\mathbf{R}$ conform to the relation

$$
G=\frac{1}{R} \text { (siemens) }
$$

(2) Elimination of electrode polarization

It is known that a conductivity greater than $10^{-10} \mathrm{~S} / \mathrm{m}$ is liable to cause electrode polarization effects at low frequencies, especially in aqueous systems (Hill et al, 1969). Ions in the immediate neighbourhood migrate to the electrode surfaces, producing localized concentrations. The net effect is that the observed impedances 
will appear larger than the values attributed to the polarization of the material itself. The electrode polarization can be eliminated by measuring the impedances under various separation distances between the electrode plates. A detailed derivation and example calculation with respect to the elimination of electrode polarization is enclosed in Appendix 3.

(3) Test cell

The test cell is composed of two parallel conducting plates separated a fixed distance. as shown in Fig.4.7. The electrode-plates are made of a print-circuit board. The conducting areas on the two plates are separated by a U-shaped separator made of Teflon, an excellent dielectric material. The electrodes and separator are held together by seven screws. The dimension of each plate is $10 \times 10 \mathrm{~cm}$. The distance between the electrode plates can be changed from $2 \mathrm{~cm}$ to $4 \mathrm{~cm}$ by using different separators.

\section{(4) Instrumentation}

The capacitance and conductance of the soils were measured with a type 1656 Impedance Bridge (General Radio Co.). This instrument is essentially an ac Wheatstone bridge with a sensitive visual null indicator. The impedances were measured at a constant frequency of $60 \mathrm{~Hz}$, generated by an Audio Signal Generator. The frequency is chosen to match that used in dielectrophoretic experiment throughout this study. Fig. 4.8 shows the test set-up.

The soil samples, either in undisturbed or remoulded slurry states, were filled in the space between the electrode-plates which in turn connected to the "Unknown" end on the bridge. The capacitance and conductance were measured by balancing 
the null indicator. Each test was repeated twice by using two separators, i.e., $2 \mathrm{~cm}$ and $4 \mathrm{~cm}$ in thickness, respectively. The first measurement was carried out in the 4-cm thickness cell. Then the $2-\mathrm{cm}$ thickness cell was installed by replacing the separator. The cell was sealed by silicon grease to avoid leaking during the measurement of dilute clay suspensions. In order to avoid errors caused by overheating the soil sample, the test duration was limited within 5 minutes for each sample.

\section{(5) Soil samples}

The Leda clays recovered from the two sites as described in Section 4.2 were tested. The undisturbed soil samples had been wax-sealed and carefully stored to preserve their in-situ moisture and structure. To prepare a test, the soil was trimmed to the size of $10 \times 10 \times 4 \mathrm{~cm}$ to fit into the test cell, as shown in Fig.4.9. Care was taken to ensure complete contact between the soil specimen and electrode-plates. After the first measurement, the soil was taken out of the cell and trimmed again to the size of $10 \times 10 \times 2 \mathrm{~cm}$ for the second measurement. Based on the data from the two measurements, the permittivity and conductivity of the soil can be calculated, as illustrated in Appendix 3.

In order to observe the dielectric properties of in-situ soils in $r$ :ation to their water contents, the undisturbed soil samples were let dry for 10 hours and measured again.

The remoulded soil samples were prepared by kneading the soil to destroy the soil structure. The clays became very soft and slurry-like once being remoulded, the characteristic of the Leda clays. The test series started from the soil at the in-situ 
water content, then distilled water was added to make the clay suspensions with different water contents ranged from $37.2 \%$ to $3683.8 \%$, corresponding to the volume fraction of clay particles from 0.49 to 0.01 , Eq.(3.32).

\subsubsection{Results}

The results of dielectric property measurement for soils at in-situ water contents are summarized in Table 4.4. The relative permittivity and conductivity in relation to the volume fraction of particles are presented in Fig.4.10 and Fig.4.11, respectively. Note that the limits of the volume fraction of clay particles are zero for pure water and 1.0 for dry soil, as defined in Eq.(3.22).

\section{(1) Remoulded clays}

The permittivity and conductivity of the remoulded Leda clays at in-situ water contents are summarized in Table 4.4. The apparent permittivity is $41.6 \times 10^{3}$ for the Gloucester clay and $24.6 \times 10^{3}$ for the Orleans clay, respectively, in good agreement with the prediction for illite, Table 3.2. The conductivity is $0.133 \mathrm{~S} / \mathrm{m}$ for the Gloucester clay and $0.083 \mathrm{~S} / \mathrm{m}$ for the Orleans clay. The effective polarizabilities of the remoulded clays can be computed according to the measured permittivity and conductivity data. The evaluation includes three steps:

(A). Permittivity. The apparent permittivities, $\epsilon_{2}$, of the two Leda clays are listed in Table 4.4. The permittivity of pore fluid is approximately constant, i.e., $K_{m}=80$ (water).

(B). Conductivity. The conductivities of the pore fluids squeezed from the clay, $\sigma_{m}$, were measured by a conductivity meter. The conductivities of the clay particles, 
$\sigma_{p}$, is in the range of $10^{-3} \mathrm{~S} / \mathrm{m}$ (Table 2.1).

(C). Determination of Polarizabilities of the clays by Eq.(3.25).

It may be seen that the values of the effective polarizubility are negative for both clays. Hence the clays would experience a dielectrophoretic force towards the direction of lower electric field intensity, i.e. pointing outwards from the central electrode. Disturbance to the clays did not affect the magnitude of the effective polarizability, indicating that the clays are located in the conductive regime. Comparing the polarizabilities of the Gloucester clay and the Orleans clay, the former is slightly higher in value, primarily due to its higher salinity of pore fluid.

The experimental results in the clay slurries show that the permittivity is proportional to the volume fraction of clay particles, Fig.4.10. The predicted relationship between the apparent permittivity and volume fraction of particles according to the theory of Dukhin and Shilov, Eq.(3.24), is illustrated by the solid lines in Fig.4.10. It may be seen that the agreement between the experimental data and the predicted relationship is excellent in both clays up to the volume fraction of particles of $p=0.3$. Thus we may conclude that the assumption of dilute suspension is valid at the limit of $p<0.3$. It should be noted that for the clay-waterelectrolyte systems with the volume fraction of particles higher than the limit, the double layer polarization mechanirm will still contribute to the events significantly. As evidenced in Fig4.10, the apparent relative permittivities of the clays remain in the order of $10^{4}$ up to the volume fraction of 0.5 even though they do not follow the linear trend.

The conductivity data in the clay slurries also show the linear trends in relation 
to the volume fraction of clay particles. Fig.4.11. The mechanism of conduction in clay-water-electrolyte systems has been discussed in Chapter 3. As evidenced from the experiment, the conductivity of a clay-water-electrolyte system is about one order lower that the conductivity of its pore water-electrolyte, due to the combined effect of electrolyte conduction and clay particle conduction. The results are consistent with previous experimental data reviewed in Chapter 3 (Table 3.1).

The permittivity and conductivity of the slurries begin to decrease after the peak values are reached, as seen in Fig.4.10 and Fig.4.11. This may be explained as follows: in a dry clay, absorbed cations are tightly held by the negatively charged clay surfaces. Cations in excess of those needed to neutralize the electro-negativity of the clay particles and their associated anions are present as salt precipitates (Mitchell, 1976). With existence of ample amount of water as saturated clays in nature, the precipitate salts go into solution and contribute to the polarization of double layer in an electric field. When the water content reduces below certain point, the ions in water-electrolyte can no longer move freely, in other word, a large portion of ions return to the state of salt precipitates. As a result, the distribution, polarization and conduction of the electrical double layer are restricted, which appear as the lower permittivity and conductivity.

It may be noted that the dielectric properties for the remoulded clays are higher than those of the undisturbed clays. This may be qualitatively interpreted from the nature of the sensitive clays. The undisturbed fabric of sensitive clays is composed of flocculated assemblages of particles or aggregates, as illustrated in Fig.4.12 (Mitchell, 1976). It is generally accepted that sensitive clays must have 
acquired an open structure during sedimentation and that this structure remained open even though subsequently buried by many meters of soil. It is suggested that a slow rate of deposition accompanied by simultaneous development of bonds is necessary for retention of the open fabric after burial. In the Leda clay, the cementation bonds are acting as a significant factor (Quigley, 1980 ). The constituents of the soils, including calcium carbonate and amorphous complex hydroxides of silica, alumina and iron, are adsorbed at particle interfaces as cementing agents to provide bonds between particles. These agents can only react to an external electric field in a limited scope due to their bonded states. Once being disturbed, these constituents in the soil are released from inter-particle connections so they are able to contribute to the polarization of double layer and conduction mechanism in a much larger scope, which appears to observers as the increase of permittivity and conductivity.

(2) Undisturbed clays

The permittivities and conductivities of the Gloucester clay and the Orleans clay at in-situ conditions are summarized in Table 4.4. The value of the apparent relative permittivity $K_{\mathrm{a}}$ is $13.5 \times 10^{3}$ for the Gloucester clay and $14.8 \times 10^{3}$ for the Orleans clay, respectively, due to the effect of double layer polarization. The apparent permittivities in the undisturbed clays are lower than the predicted value for illite, Table 3.2, suggesting that the soil structure would restrict the double layer polarization.

The soil conductivity is $0.067 \mathrm{~S} / \mathrm{m}$ for the Gloucester clay and $0.040 \mathrm{~S} / \mathrm{m}$ for the Orleans clay, correspending to the pore fluid conductivities of $0.252 \mathrm{~S} / \mathrm{m}$ and 
$0.140 \mathrm{~S} / \mathrm{m}$, respectively, as shown in Table 4.4.

The polarizability of the Leda clays at undisturbed condition was evaluated as discussed before. The results are summarized in Talse 4.4. It may be seen again that the polarizabilities of both clay soils at undisturbed states are negative and not affected by disturbance.

The permittivities and conductivities of the undisturbed clays reduced after 10 hour natural drying, as seen in Fig.4.10 and Fig.4.11. The same discussion as that for the remoulded clay may interpret the phenomenon.

\subsubsection{Summary and Conclusion}

The permittivity and conductivity of clay-water-electrolyte systems are key electrical properties in dielectrophoresis. The technique described in this section proved to be successful in determining the two properties. The following conclusions may be drawn from the investigation to the Gloucester clay and Orleans clay of Leda clay origin:

(1). The clays exhibited extraordinary high apparent permittivity in an external electric field. This behaviour may be explained by the theory of double layer polarization;

(2). The apparent permittivities of a clay in undisturbed and remoulded states at the same water content were significantly different;

(3). The apparent permittivity of remoulded clays was proportional to the volume fraction of particles in dilute suspensions up to certain limit, in agreement with the theory of double layer polarization; 
(4). The conductivities of the Leda clays were about 1 order lower that the conductivities of their pore fluids.

(5). The conductivity and permittivity of the Leda clays were decreased as the water contents reduce to a point from which the ion mobility is restricted.

(6). The effective polarizabilities estimated from the data of apparent permittivity and conductivity of the Leda clays suggest that the clays would experience the negative dielectrophoresis, i.e., the dielectrophoretic force directed towards the lower field intensity in a non-uniform electric field. The magnitude of the polarizability is not affected by the disturbance to the soils. 
TABLE 4.1 SUMMARY OF SOIL PROPERTIES

\begin{tabular}{|c|c|c|}
\hline SOIL PROPERTY & $\begin{array}{l}\text { GLOUCESTER } \\
\text { CLAY }\end{array}$ & $\begin{array}{l}\text { ORLEANS } \\
\text { CLAY }\end{array}$ \\
\hline Depth, $m$ & $2 \cdot 6$ & $4.2-4.5$ \\
\hline Water Content, \% & $60-90$ & $68 \cdot 74$ \\
\hline Liquid Limit, \% & $43-56$ & $63 \cdot 70$ \\
\hline Plastic Limit, \% & $21-26$ & $31 \cdot 39$ \\
\hline Plastic Index & $22-30$ & $31-32$ \\
\hline \% Finer than $2 \mu \mathrm{m}$ & 70 & 64 \\
\hline Liquidity Index & $1.7-2.1$ & $1.1-1.2$ \\
\hline Sensitivity & $20-100$ & $40-60$ \\
\hline Hydraulic Permeability, $\mathrm{cm}^{2} / \mathrm{s}$ & $2.2 \times 10^{-8}$ & $2.9 \cdot 3.1 \times 10^{-8}$ \\
\hline Brittleness Index & 0.39 & $0.43-0.69$ \\
\hline Undrained Modulus, MPa & 1.7 & $5.4 \cdot 6.2$ \\
\hline Activity & $0.31 \cdot 0.43$ & $0.49 \cdot 0.51$ \\
\hline Unit Weight, $\mathrm{kN} / \mathrm{m}^{3}$ & 16.2 & 16.3 \\
\hline Pre-consolidation Pressure, $k \mathrm{~Pa}$ & $35 \cdot 87$ & $142 \cdot 146$ \\
\hline Over Consolidation Ratio & 1.5 & 2.4 \\
\hline Specific Gravity & 2.78 & 280 \\
\hline Unconfined Shear Strength, $k P a$ & $12.6-17.9$ & $61.7-68.8$ \\
\hline Vane Strength, $k P a$ & 15.7 & 68.6 \\
\hline Void Ratio & 2.2 & 1.9 \\
\hline
\end{tabular}

* After Ho, 1990. 
TABLE 4.2. SUMMARY OF CHEMICAL ANALYSIS

\begin{tabular}{lll}
\hline SOIL PROPERTY & $\begin{array}{l}\text { GLOUCESTER } \\
\text { CLAY }\end{array}$ & $\begin{array}{l}\text { ORLEANS } \\
\text { CLAY }\end{array}$ \\
\hline Carbonace Content, \% & 2.6 & 1.1 \\
Salinity, $g / l$ & 1.26 & 0.7 \\
Pore Fluid Conductivity , $m S / m$ & 252 & 140 \\
$\mathrm{Cl}, m g / l$ & 240 & 225 \\
$S O{ }^{2}, m g / l$ & 20 & 20 \\
pH & 8.6 & 8.1 \\
$K_{2} O, \%$ & 3.30 & 3.48 \\
C.E.C. , meq $/ 100 \mathrm{~g}$ soii & 19.8 & 16.3 \\
\hline
\end{tabular}

TABLE 4.3. SUMMARY OF COMPOSITION DATA

\begin{tabular}{lll}
\hline MINERAL CONTENT, \% & $\begin{array}{l}\text { GLOUCESTER } \\
\text { CLAY }\end{array}$ & $\begin{array}{l}\text { ORLEANS } \\
\text { CLAY }\end{array}$ \\
\hline Illite & $55-60$ & $55-60$ \\
Chlorite & $10-15$ & $5-10$ \\
Quartz & 17 & 14 \\
Feldspar & $2-3$ & $7-10$ \\
Amphibole & $<5$ & $5-6$ \\
\hline
\end{tabular}


TABLE 4.4 DIELECTRIC PROPERTIES OF LEDA CLAYS

\begin{tabular}{lll}
\hline PROPERTIES & $\begin{array}{l}\text { GLOUCESTER CLAY } \\
\mathrm{w}_{\mathrm{n}}=63.8 \% \\
\mathrm{p}=0.36\end{array}$ & $\begin{array}{l}\text { ORLEANS CLAY } \\
\mathrm{w}_{\mathrm{n}}=69.0 \% \\
\mathrm{p}=0.34\end{array}$ \\
\hline Relative Permittivity & & \\
\hline$\left(\mathrm{K}_{\mathrm{s}}\right)_{\mathrm{u}} \times 10^{3}$ & 13.5 & 14.8 \\
$\left(\mathrm{~K}_{\mathrm{g}}\right)_{\mathrm{r}} \times 10^{3}$ & 41.6 & 24.6 \\
\hline Conductivity & & \\
\hline$\sigma_{\mathrm{u}}, \mathrm{S} / \mathrm{m}$ & 0.067 & 0.040 \\
$\sigma_{\mathrm{r}}, \mathrm{S} / \mathrm{m}$ & 0.133 & 0.083 \\
$\sigma_{\mathrm{m}}, \mathrm{S} / \mathrm{m}$ & 0.252 & 0.140 \\
\hline Relative Efrective & & \\
Polarizability & & -118.7 \\
\hline$\left(\sigma_{\text {Kett }}\right)_{\mathrm{u}}$ & -119.3 & -118.7 \\
$\left(\sigma_{\text {Keft }}\right)_{\mathrm{r}}$ & -119.3 & \\
\hline
\end{tabular}

Notations:

$w_{n}: \quad$ natural water content;

p : volume fraction of clay particles;

$\left(K_{4}\right)_{8}$ : apparent relative permittivity of undisturbed soil;

$\left(\mathbf{K}_{\text {, }}\right)_{\mathrm{r}}$ : apparent relative permittivity of remoulded soil;

$\sigma_{u}: \quad$ conductivity of undisturbed soil;

$\sigma_{\mathrm{r}}: \quad$ conductivity of remoulded soil;

$\sigma_{m}: \quad$ conductivity of pore fluid medium;

$\left(a_{\text {Keff }}\right)_{u}: \quad$ relative effective polarizability of undisturbed soil;

$\left(\sigma_{\text {Kerr }}\right)_{r}: \quad$ relative effective polarizability of remoulded soil. 

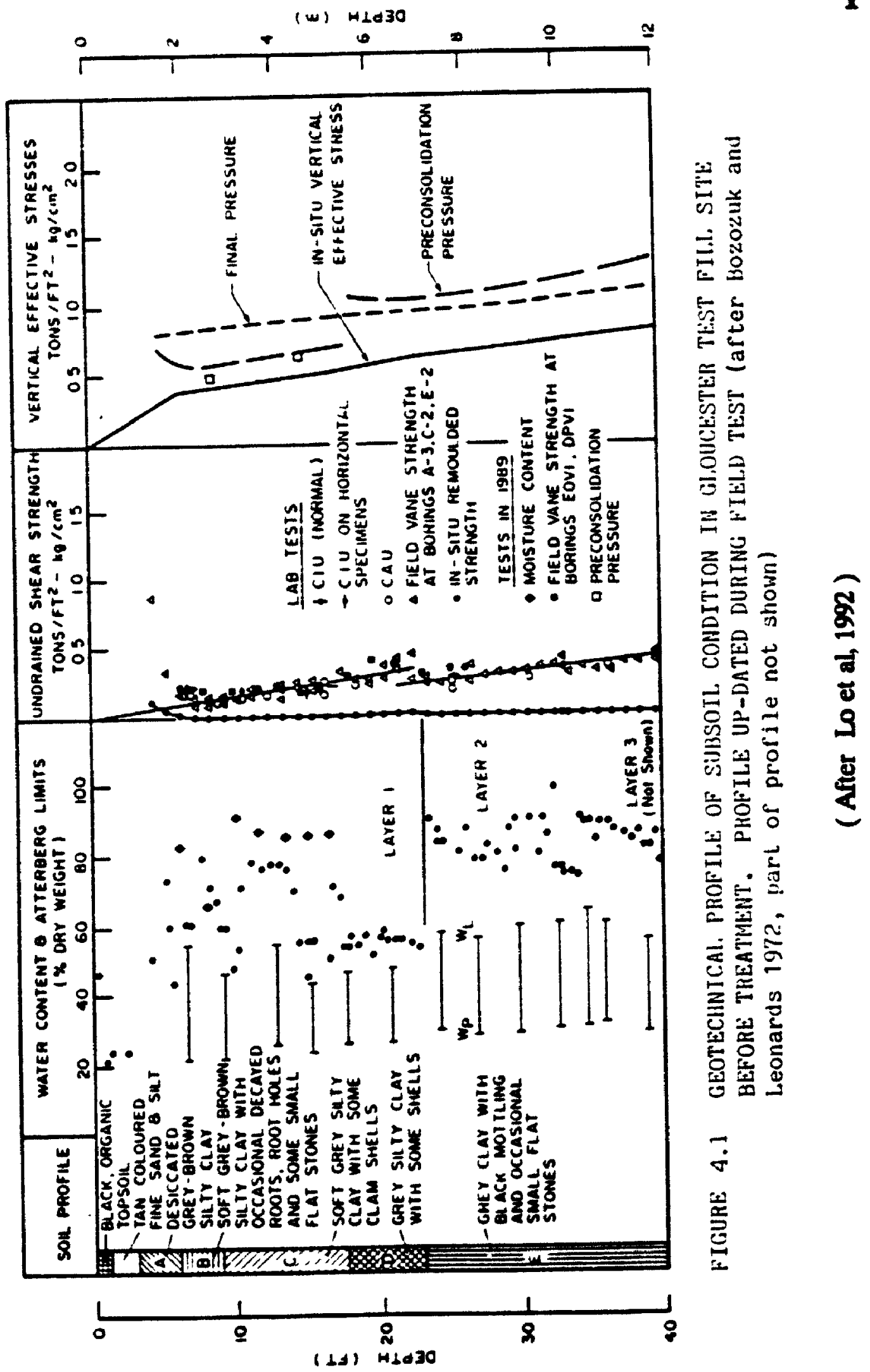
116

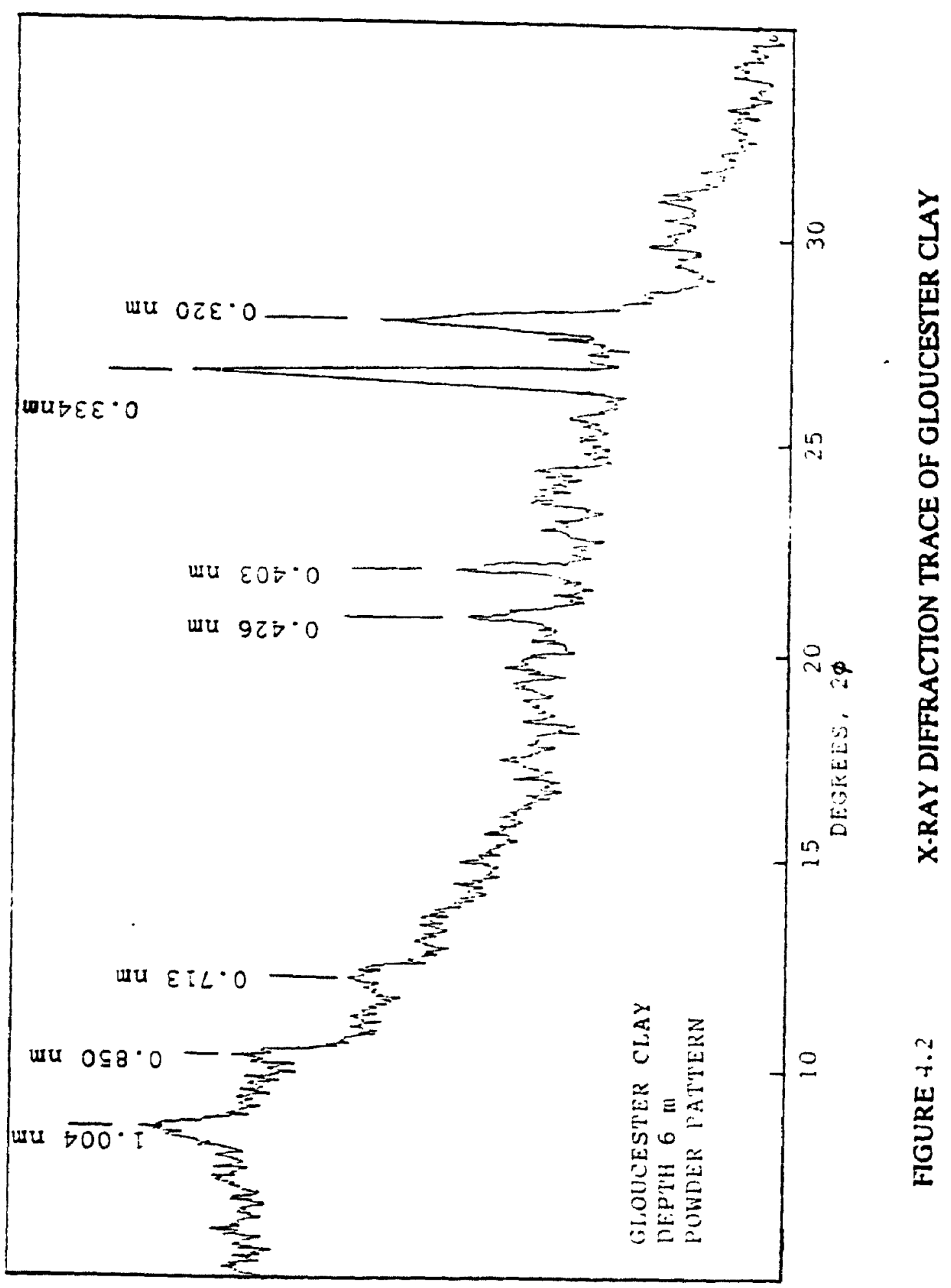




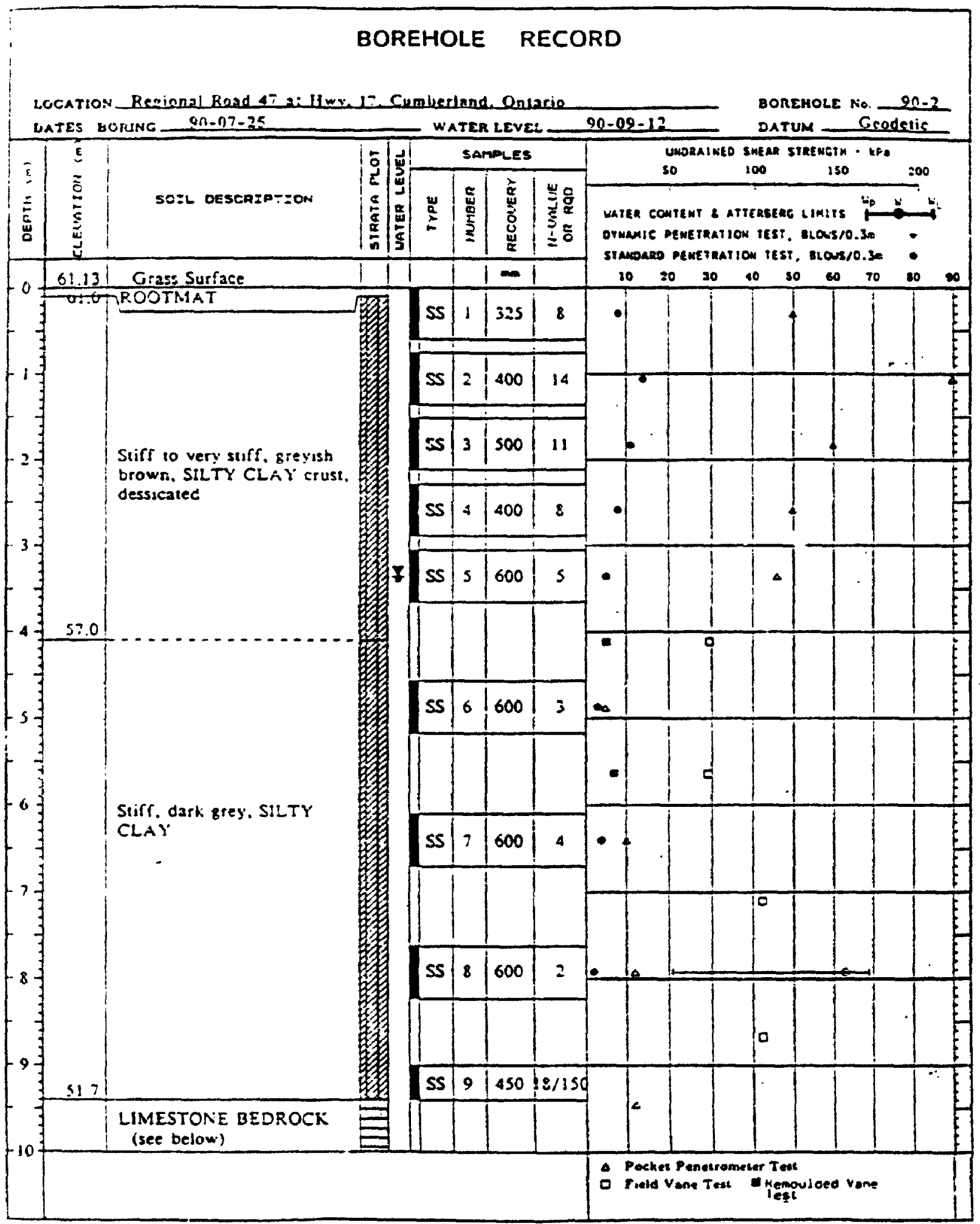

FIGURE 4.3 


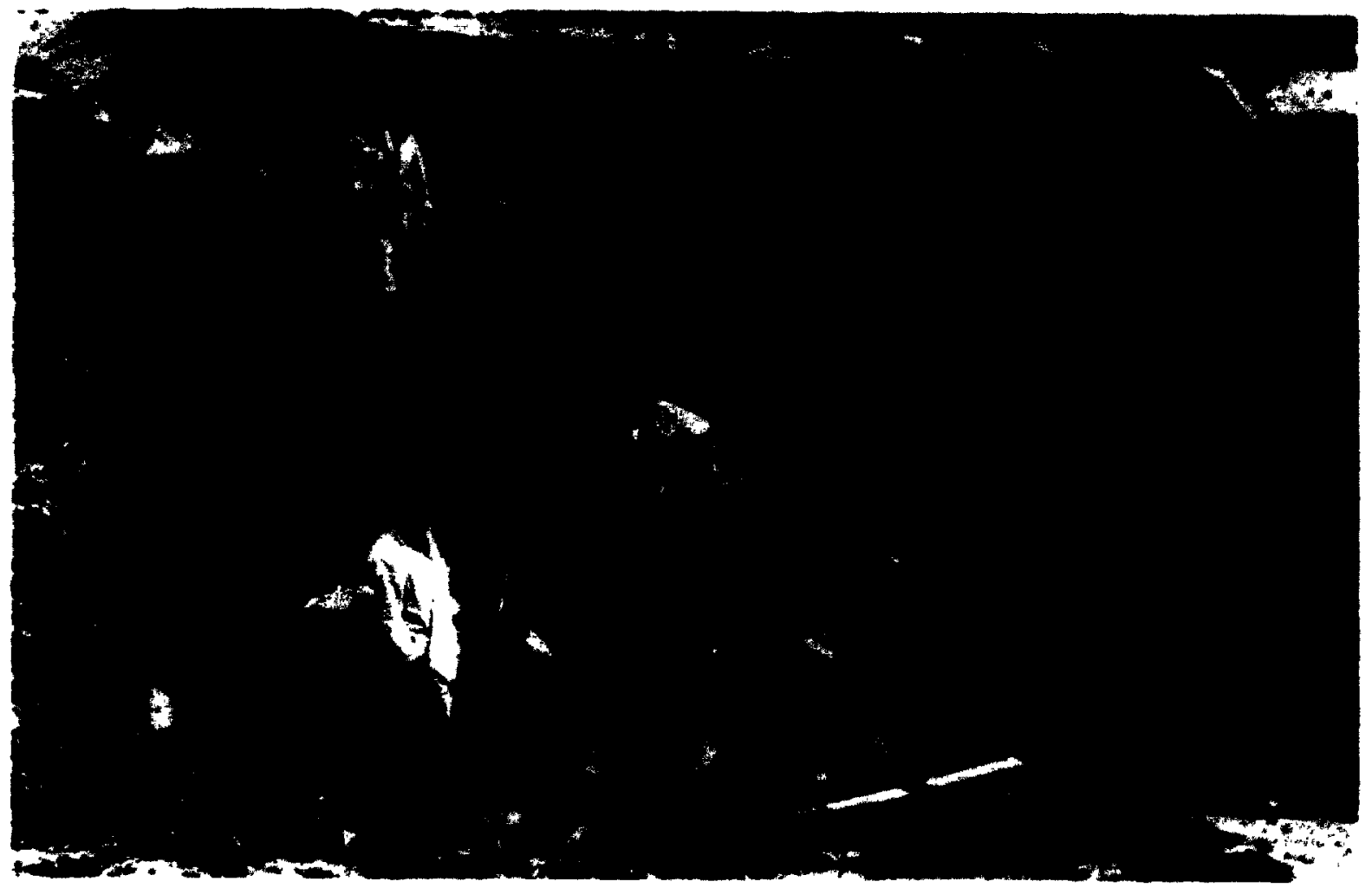

FIGURE 4.4 BLOCK SAMPLING: overview of excavation 


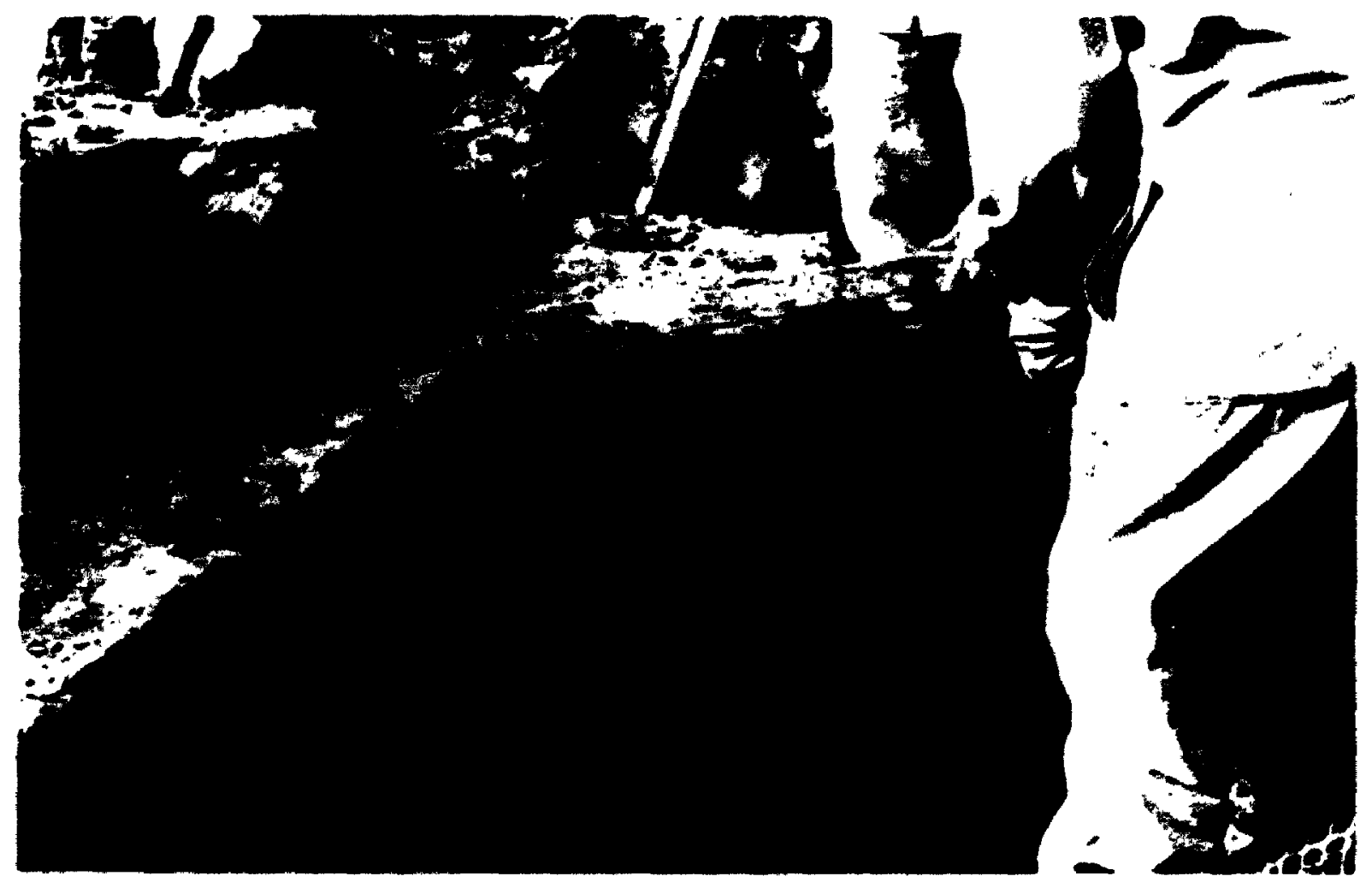

FIGURE 4,5 A CLOSE VIEW TO BLOCK SAMPLING 


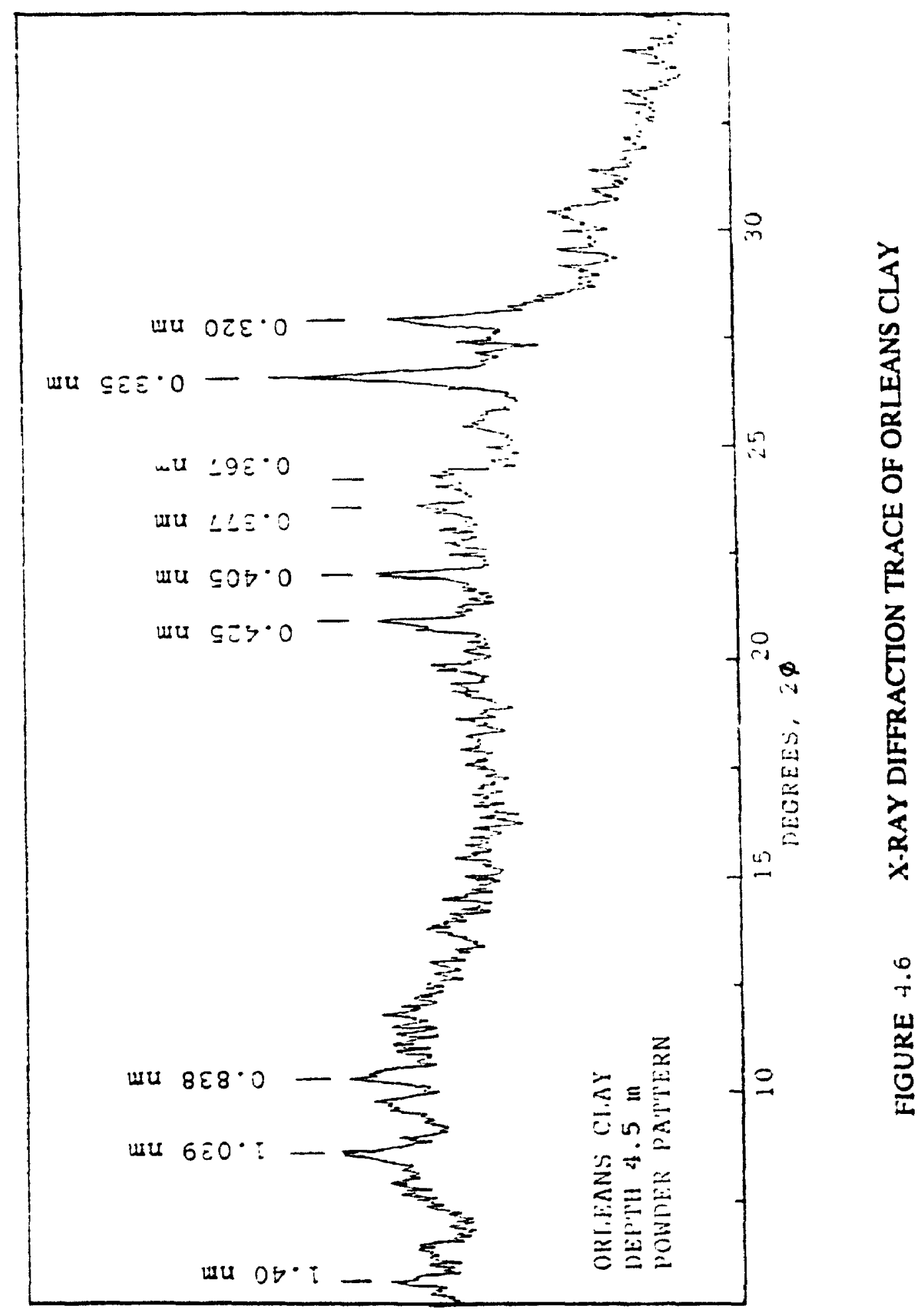



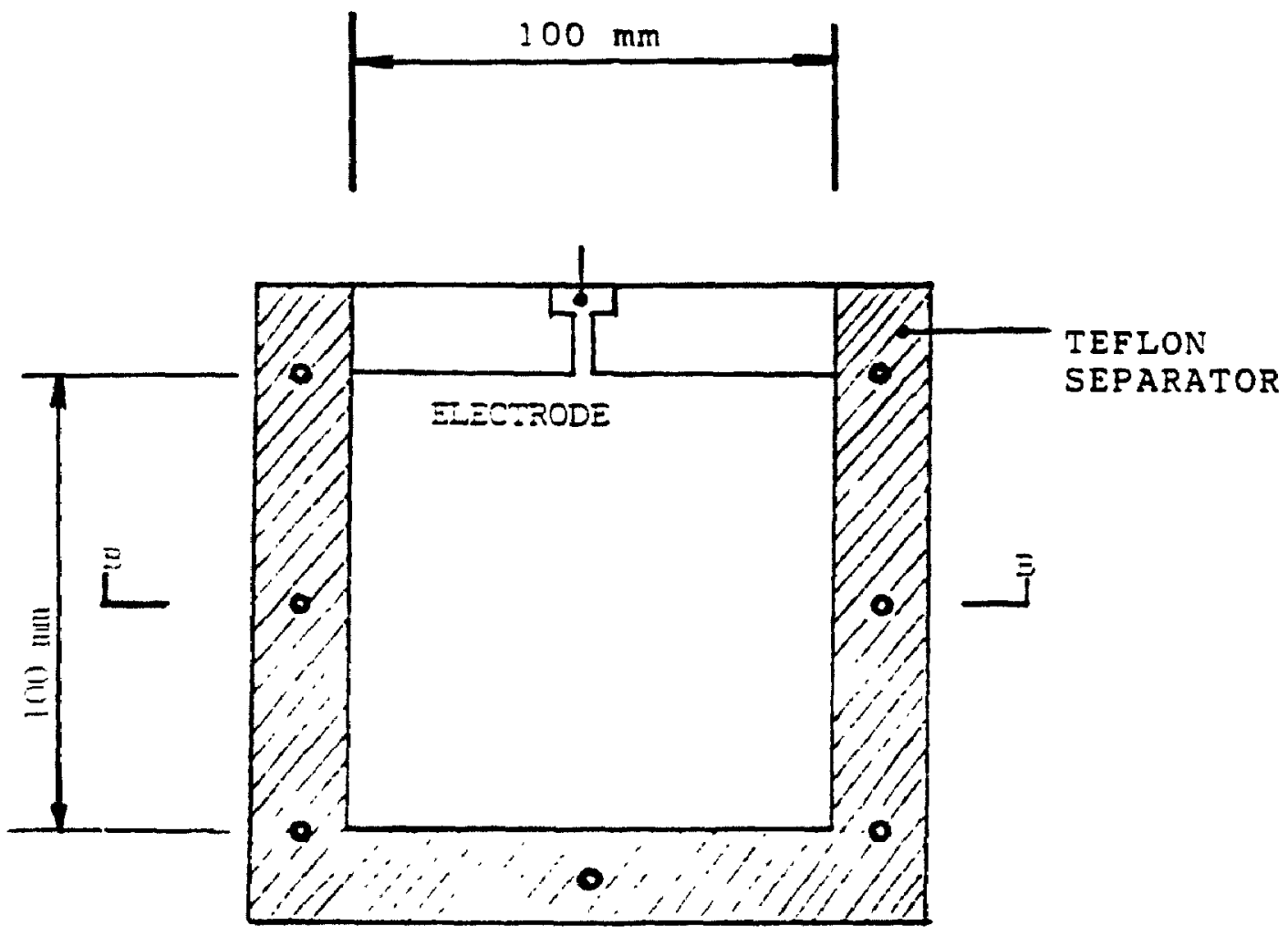

(A) A-A SECTION VIEW

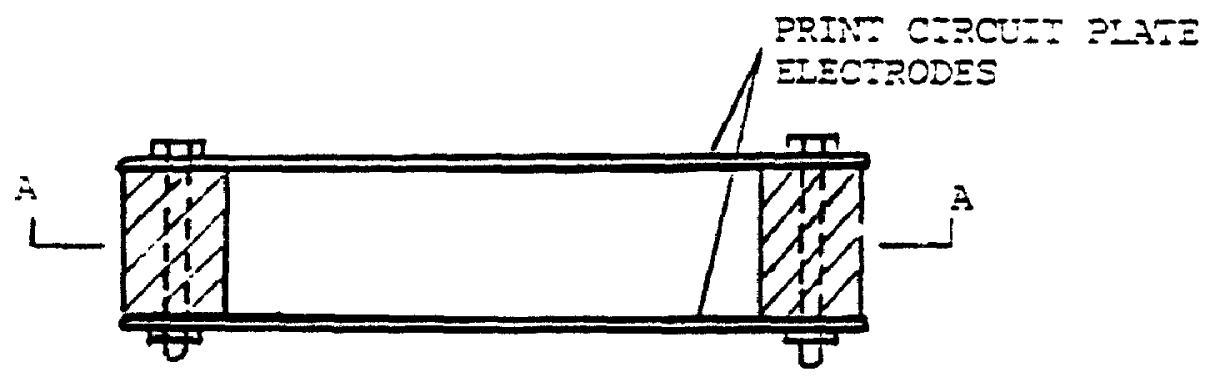

(B) B-E SECION VIEN

FIGURE 4.7 TEST CELI 


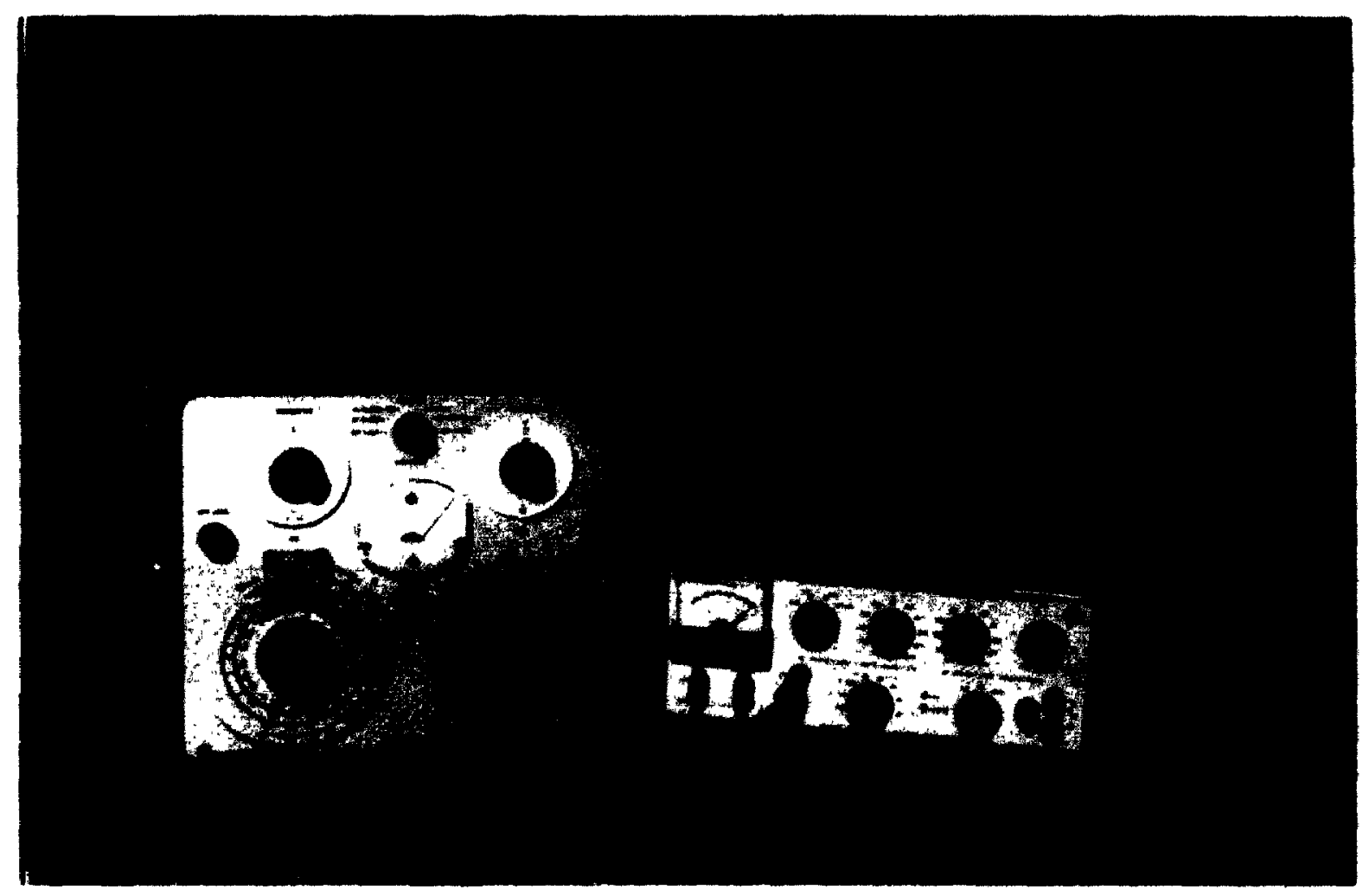

FIGURE 4.8 TEST SET-UP: MEASUREMENT OF SOIL ELECTRICAL PROPERTIES 


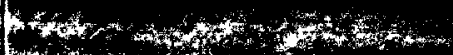

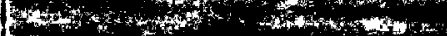

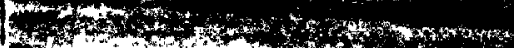

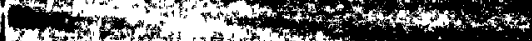

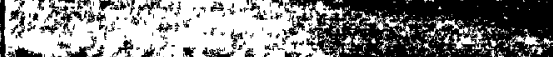

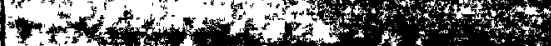

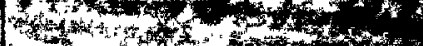

-

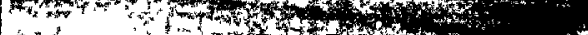

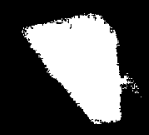

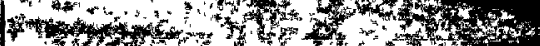

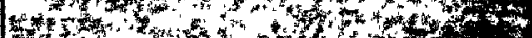

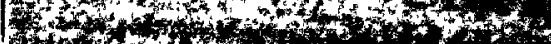

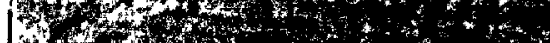

whothents

5.

$x_{i=18}$

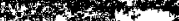

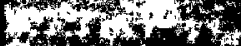

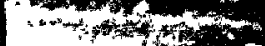

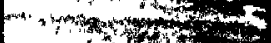

$\therefore$ (3)

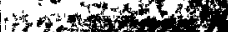

1010

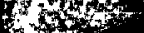

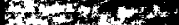

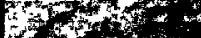

D.t.
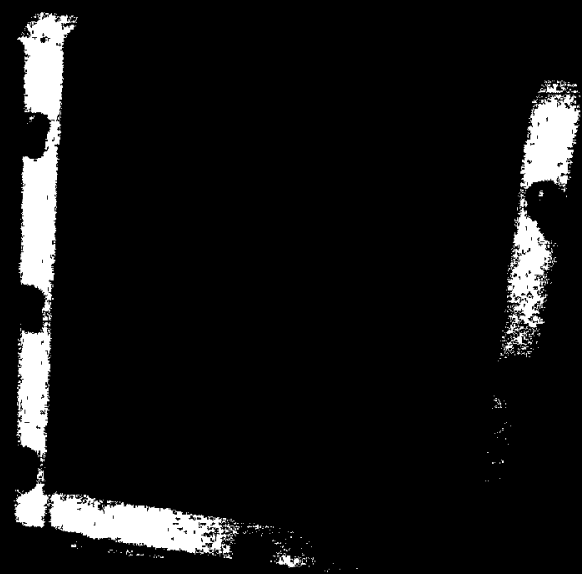

FIGURE 4.9

TEST CELL FOR MEASUREAENT OE SOIL ELECTRICAL PROPERTIES 


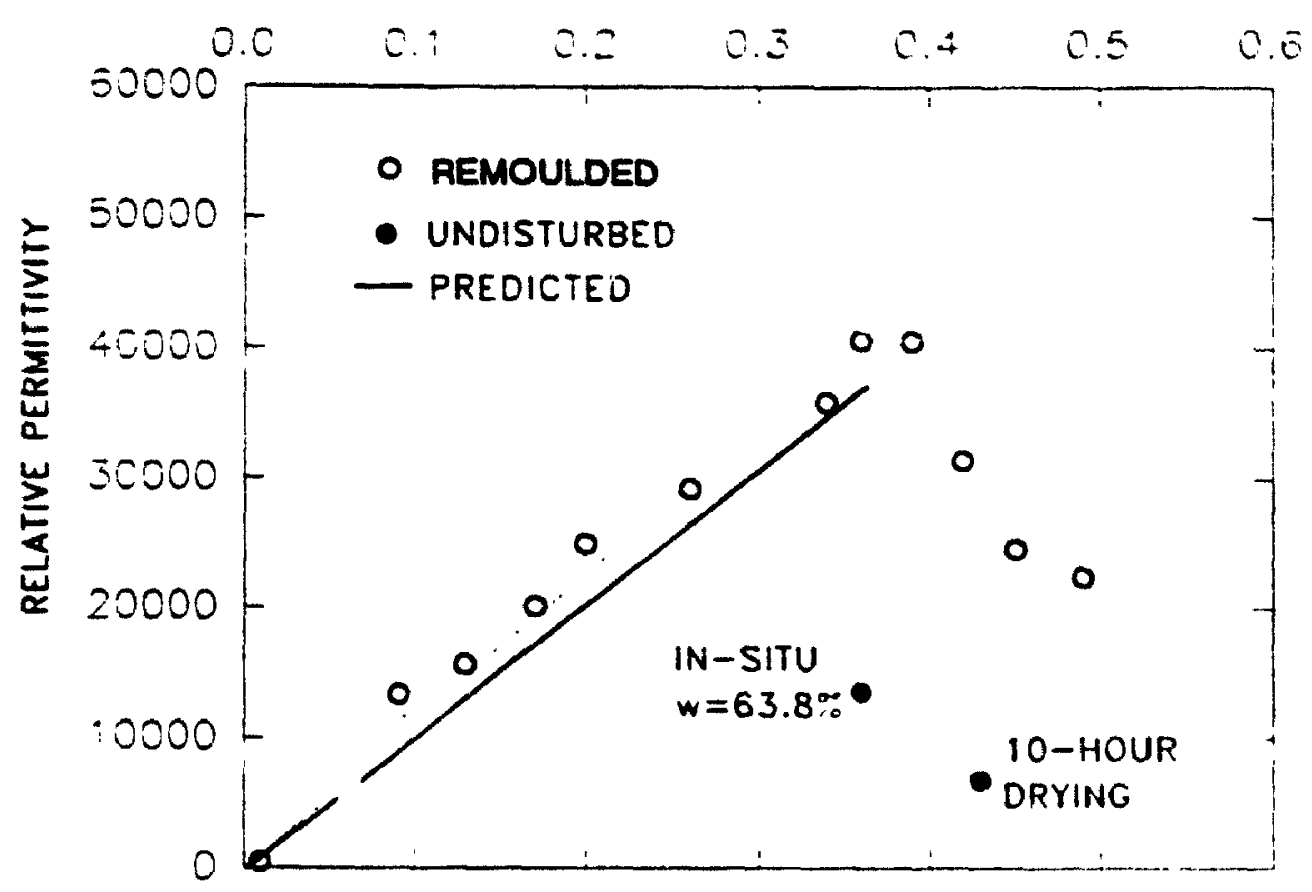

A) GLOUCESTER CLAY

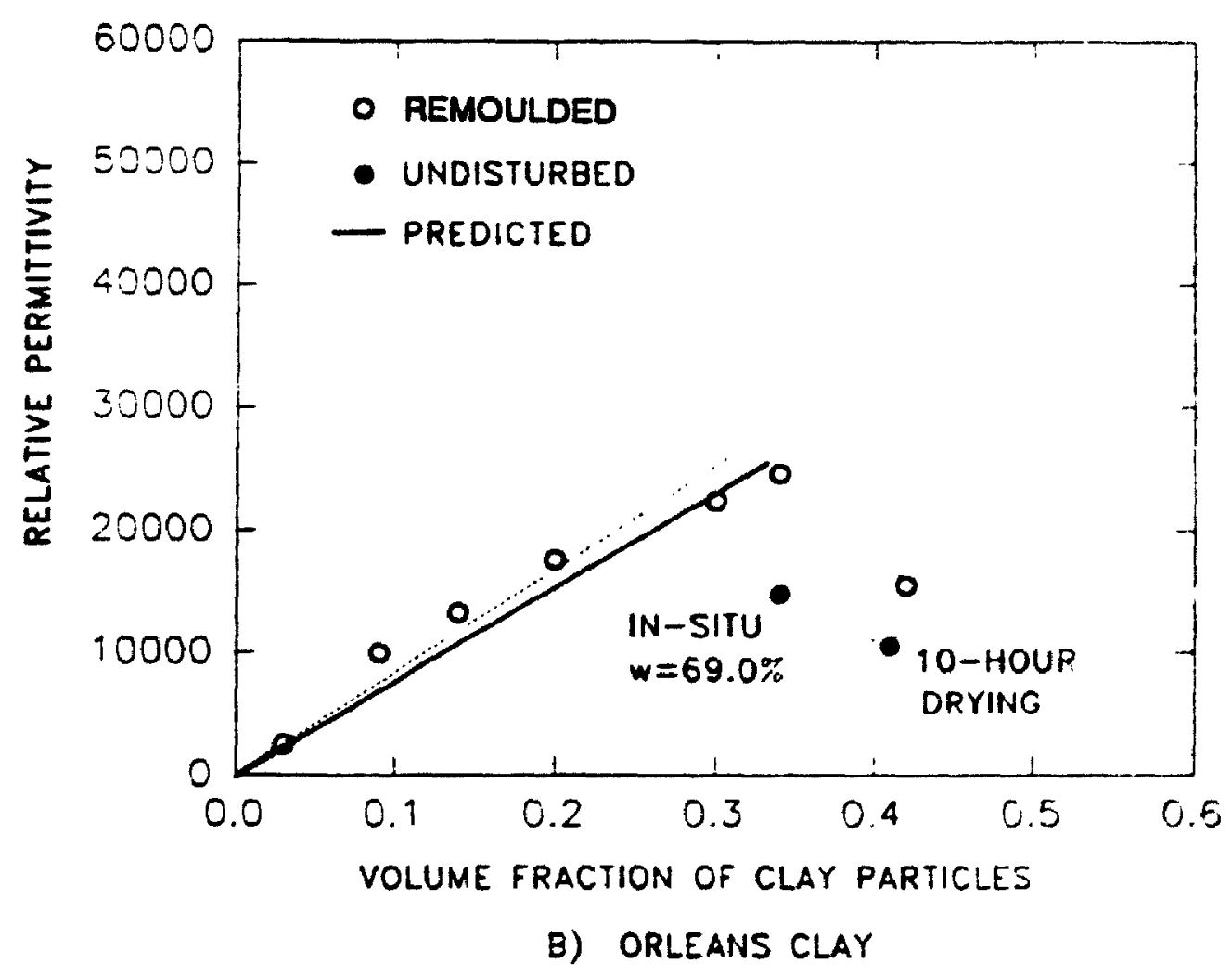

FIGLPE 4.10 APPARENT PEPMITTIVITY OF TWO LEDA CLAYS 


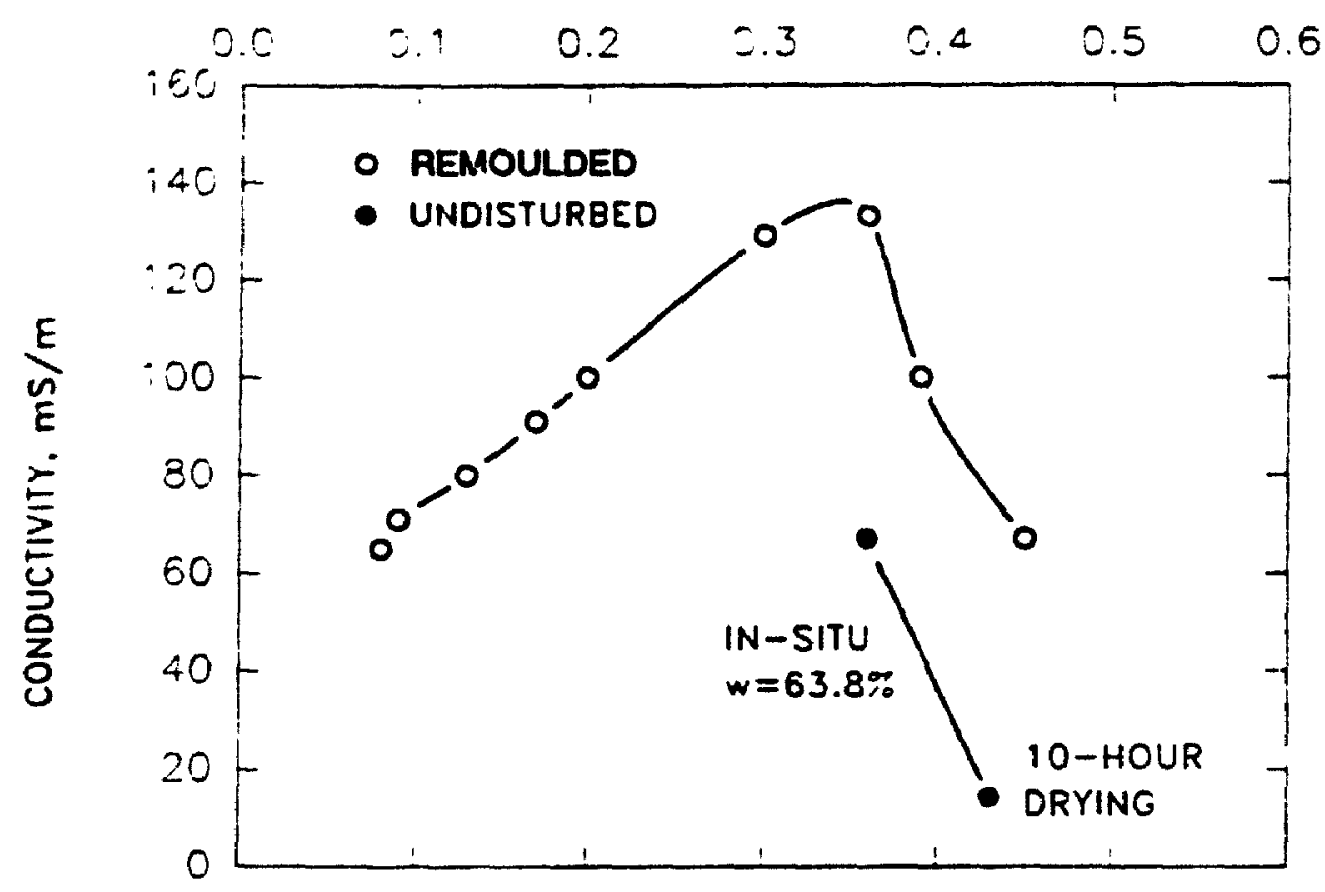

A) GLOUCESTER CLAY

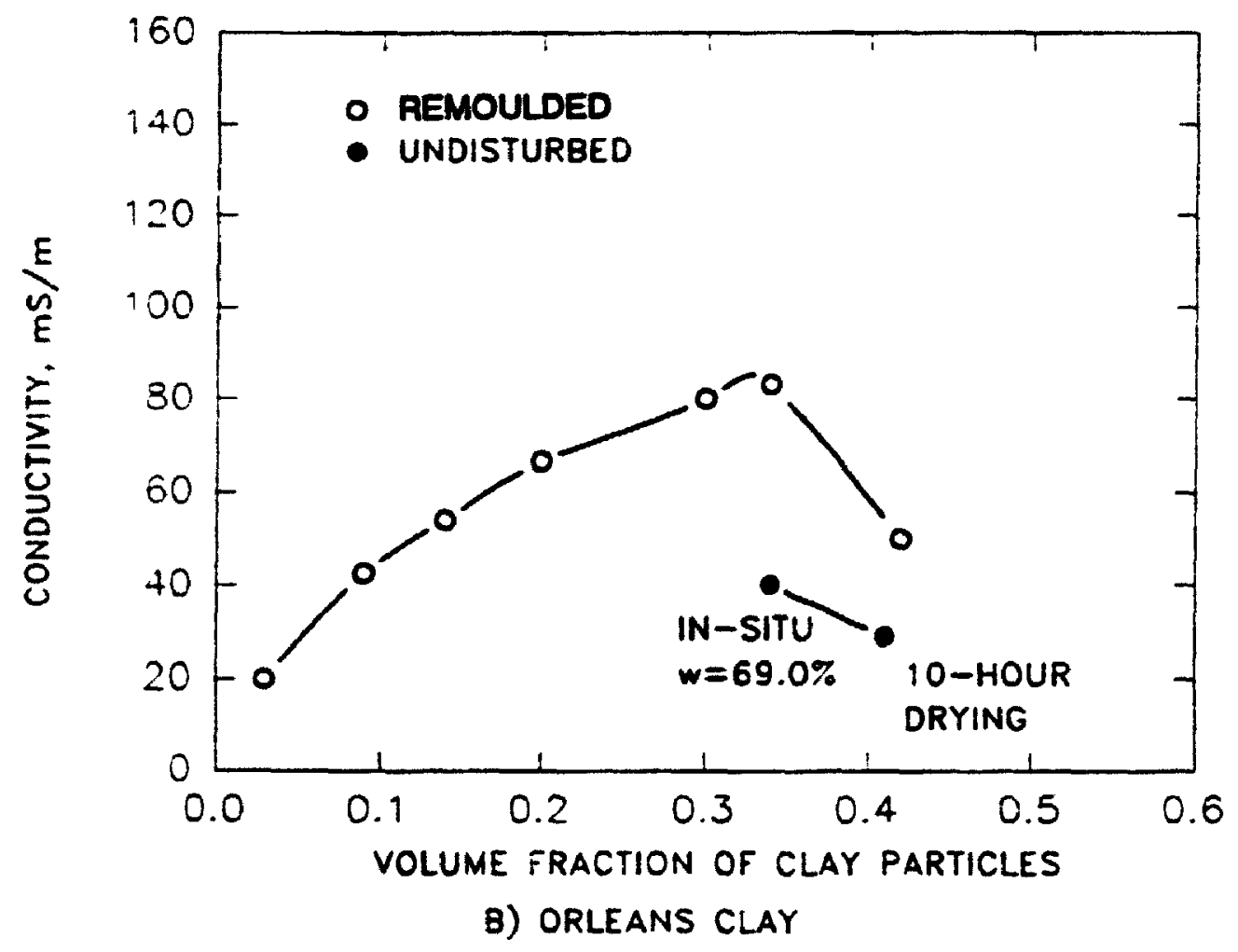

FIGURE 4.11 CONDUCTIVITY OF TWO LEDA CLAYS 


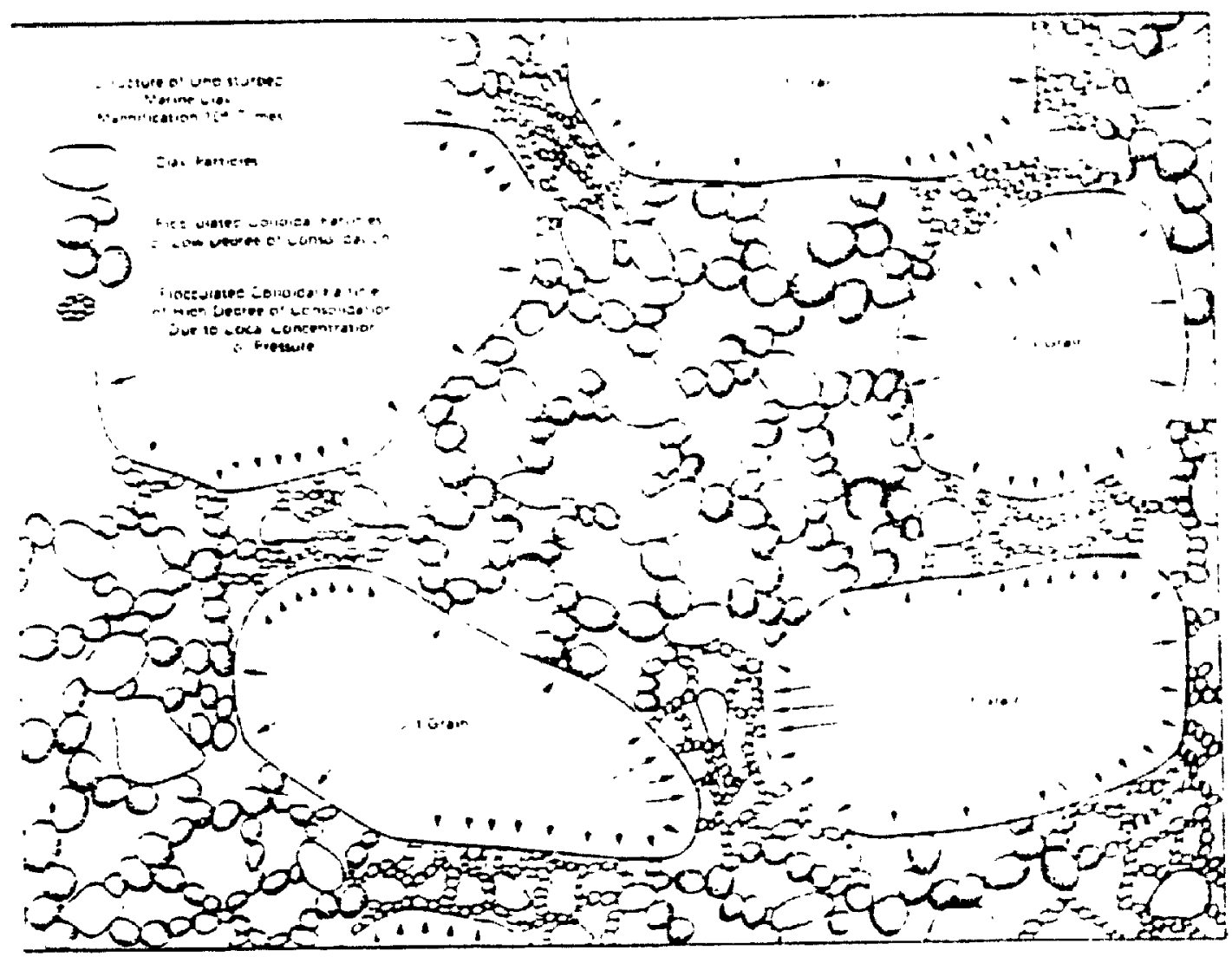

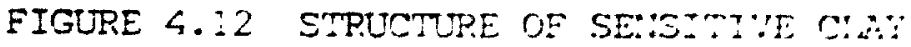

(AFTEP YITCEEL, :S76) 


\section{CIIAPTER 5}

\section{DIELECTROPHORETIC EXPERIMENTS IN GLOUCESTER CLAY}

\subsection{Introduction}

The experimental program in the Gloucester clay continued the research conducted by Inculet and Lo (1988) and Lo et al (1992) reviewed in Chapter 2, Sections 2.4.2 and 2.4.3. The effectiveness of dielectrophoresis in relation to the electrode depth and applied voltage has been understood. The electrodes were installed in the air-dry holes drilled on the soil samples. Ho (199v) has shown in a field trial test that this arrangement is not practical as it is almost impossible to maintain an air dry hole to surround the electrode where the soil layer for treatment is below the ground water table. Furthermore, the effect of the intervening medium between the electrode and the soil on the effectiveness of dielectrophoresis remain to be determined.

The experimenta! results obtained from dielectrophoresis treatment on the Gloucester clay samples are reported in this chapter with focus on the effectiveness of dielectrophoresis in relation to the intervening media filled in the central hole.

\subsection{Apparatus}

The apparatus used in the test series consisted of three basic components, the test camber, the power supply and the electrodes. A schematic diagram of the test apparatus is shown in Fig.5.1. 


\subsubsection{Test Chamber}

The soil samples were placed inside a test chamber in order to eliminate the effect of natural drying during test and to provide ventilation. The chamber was made of $6 \mathrm{~mm}$ thick plexiglas plate, $62 \mathrm{~cm} \times 62 \mathrm{~cm} \times 62 \mathrm{~cm}$ in dimension. The air intake of the chamber was connected to a humidifier. An electric powered fan was installed at the air outlet of the chamber for ventilation. The relative humidity in the chamber was controlled in the range of $50-60 \%$. In the first series of test run in parallel with two actual tests, the results showed that the effect of natural drying on a wax covered soil sample was negligible during a period of $\mathbf{3 0}$ days.

\subsubsection{Power Supply}

The power supply consisted of an audio signal generator and a $30 \mathrm{kV}$ capacity amplifier/transformer. The processing frequency was $60 \mathrm{~Hz}$. The transformer is equipped with a volt meter and an ampere meter to monitor the operation during processing.

Two soil samples were connected in paraiiel to the power supply. Tre voltage applied in all tests was $18 \mathrm{kV}$.

\subsubsection{Electrodes}

The central electrode was identical as those used by Inculet and Lo (1988), and Lo et al (1992), as shown in Fig.2.8. The ground electrodes were made by $2 \mathrm{~mm}$ diameter copper bars. The cylindrical electric field geometry was composed of the central electrode and the surrounding 12 ground electrodes connected to each 
other, as shown in Fig.5.2. The length of the electrodes inserted into a soil sample was $170 \mathrm{~mm}$. The distance between the central electrode and the ground electrode was approximately equal to the radius of the soil samples, namely, $60 \mathrm{~mm}$.

\subsection{Sample Preparation}

The Gloucester slay used in the test series has been described in detail in Chapter 4. The samples were $127 \mathrm{~mm}$ diameter Shelby tube samples, $203 \mathrm{~mm}$ in length. Fig. 5.3 presents a profile of in-situ water content and undrained shear strength of the samples along the depth.

For test preparation, a circular hole approximately $30 \mathrm{~mm}$ in diameter was cut on top centre of the w:x seal to expose the soil. Since the other strength tests were restricted due to the limited sample size, a laboratory vane (the PILCON Hand Vane Tester, English Drilling Equipment Co. Ltd.) of $15 \mathrm{~mm}$ diameter by $30 \mathrm{~mm}$ length was used to measure the undrained shear strength. The vane was calibrated by the results of the unconfined compression tests. After the vane test, a stainless steel tube with sharp cutting edge was pushed vertically into the soil along the path of vane test, making a hole of $25.4 \mathrm{~mm}$ in diameter in the centre of the sample for installing the electrode, as shown in Fig.5.2. The depth of the hole was $170 \mathrm{~mm}$, leaving $30 \mathrm{~mm}$ soil on the bottom of the sample intact. The natural water content of the sample was measured on the soil recovered out by the tube, at the top, middle and bottom of each sample. The average was taken as the in-situ water content of the sample.

The intervening medium included air (dry hole), water and slurries made of 
Gloucester clay suspensions as described in Chapter 4. The water contents of the slurries were determined prior testing.

After the fluid was poured into the hole, the central electrode was installed. supported by a plexiglas holder, as shown in Fig.5.4. The tip of the electrode was just in touch with the bottom of the central hole. The copper ground electrodes were pushed into the soil at equal distance surrounding the circumference of the sample and connected to one another, as shown in Fig.5.2. The depth of the ground electrodes was the same as that of the central electrode, i.e., $170 \mathrm{~mm}$. A narrow strip of wax was removed in the radial direction and two plastic settlement monitoring pins, $2 \mathrm{~mm}$ in diameter and $20 \mathrm{~mm}$ in length, were set on top of the sample, one beside the central hole and the other beside the edge of the sample, respectively, Fig.5.2(B). The photograph in Fig.5.4 shows the samples with completed test preparation.

\subsection{Procedure}

Two samples with different intervening medium were tested in each operation. The central electrodes were connected in parallel to the high voltage output terminal, hence the potential applied on the two samples were identical. The ground electrodes were connected to the ground return of the power supply which in turn connected to a firm ground. A potential of $18 \mathrm{kV}$ was applied on the central electrodes after all connections were completed and checked. The test chamber was closed and the humidifier and the ventilation fan were switched on. A telescope with accuracy of $0.01 \mathrm{~mm}$ was aimed on the pins to monitor the settlements of the 
samples.

Each test lasted 21 days. The samples were visually observed closely during testing period. The settlements of the samples were recorded every day. The water content and shear strength of the samples were tested upon completion of a test.

\subsection{Results}

The test results are discussed with respect to three aspects: (1). the settlement of soil samples; (2). the decrease of water contents and (3). the increase of undrained shear strength. The effects of soil water contents and different media in the central hole are investigated. Table 5.1 summarizes the test results.

\subsubsection{Settlement}

The results of settlement during a test is shown in Fig.5.5, in which the settlements of the sample at the centre and edge of the soil sample are plotted against treatment time. It may be seen that the sample settled in a rather uniform manner, without significant difference between the centre and edge. The average of the settlements at day-21 is taken as the final settlement. The sample settlements ranged from $0.8 \mathrm{~mm}$ to $2.5 \mathrm{~mm}$, as shown in Table 5.1, corresponding to the vertical strain ranging from $0.4 \%$ to $1.2 \%$.

\subsubsection{Change of Soil Water Content}

The water contents of the soil sample: after treatment were tested in 9 points throughout the soil sample to obtain an average. The change of water content 
is defined as

$$
\Delta w=\frac{w_{e}-w_{n}}{w_{n}} \times 100 \%
$$

where

$\Delta w=$ change of soil water content after treatment;

$w_{n}=$ natural soil water content;

$\mathbf{w}_{\mathbf{a}}=$ soil water content after treatment.

We may see from Table 5.1 that the water content decrease was observed in every sample, ranging from $3.4 \%$ to $26.3 \%$. Fig. 5.6 shows the change of water content in relation to the water contents of medium in the cylindrical space between the electrode and the sample. It may be seen that the soil water content decreases as the initial water content of the slurry increases. The test with "dry" hole showed the lowest effectiveness in term of decrease of water content. Tests with the hole filled with tap water also shows the process was not effective.

The change of the medium in the cylindrical space after treatment was examined. For the sample with the central hole filled with water, the water was completely dried out. The central electrode was clean, without any trace of clay particle attachment. For the sample in which the decrease of water content was less significant (Test G18K-3.2, $\Delta w=4.6 \%$, Table 5.1), the slurry was thickened (the water contents were reduced), whereas for those with significant decrease of water content, $(\Delta w>10 \%)$, the water contents of the slurry became the same or less than those of the soil samples. 


\subsubsection{Change of Soil Shear Strength}

The change of soil shear strength after dielectrophoretic treatment is defined is

$$
\Delta c_{n}=\frac{c_{m}-c_{u n}}{c_{u n}} \times 100 \%
$$

where

$\Delta c_{\mathrm{u}}: \quad$ change of undrained shear strength after dielectrophoretic treatment;

$c_{\text {un }}$ : undrained shear strength before treatment;

$c_{u a}: \quad$ undrained shear strength after treatment.

The results of shear strength measurement before and after treatment are summarized in Table 5.1. It should be noted that the measurement of shear strength can only provide comparison on a relative bases due to the limited sample size.

The change in shear strength in relation to the water content of the intervening medium is presented in Fig.5.6. It may be seen that as the water content of the slurry decreases, the strength after treatment increases. This observation is consistent with the trend of soil water content change shown in the same figure.

The sample with air-dry and water filled holes present the lowest increase of shear strength ( $4.1 \%$ and $5.2 \%$, respectively), comparing to an increase of $37.5 \%$ at slurry water content of $118.5 \%$, Table 5.1.

Again we find here that the best dielectrophoresis effectiveness for increasing soil shear strength may be achieved by using a "thick" clay slurry filling in the hole of electrode installation. 


\subsubsection{Summary}

The results in the Gloucester clay samples suggests that the action of dielectrophoresis would be improved by using a slurry of low water content. The maximum effectiveness would be the case of electrode in direct contact with soil. We may conclude hence that by using a slurry made of same clay soil with minimum water content to fill the hole for electrode installation, we may obtain the best effectiveness in dielectrophoresis process. We can also minimize the diameter of the hole to increase the effectiveness of dielectrophoresis treatment.

\subsection{Effect of Intervening Media on Electric Field Intensity}

The experimental study on the Gloucester clay samples has provided some important indications with respect to the effectiveness of dielectrophoresis in clays. In this section, the effect of electric field intensity as affected by the intervening media is discussed and compared with the experimental data.

The distributions of cylindrical electric fields discussed in Chapter 3 are the ideal case in terms of electrode installation, i.e., the electrode is in contact with the medium, as shown in Fig.3.1. In engineering practice, however, a hole must be drilled prior to install the electrode. Thus an intervening medium would be present between the electrode and soil mass. As a result, the electric field intensity distribution will be affected by the dielectric properties of both the soil and the intervening material filled in the hole.

Consider the case of perfect cylindrical electric field geometry, as shown in Fig.5.7. Similar to the discussion in Chapter 3, Section 3.2, the central electrode has 
a metallic rod of radius $R_{a}$ and an insulation layer of radius $R_{b}$. The outer electrode has the radius $R_{c}$ A hole of radius $R_{h}$ is located in the centre of the cylinder, filled with an intervening material of permittivity $\epsilon_{\mathrm{b}}$, as shown in Fig.5.7. Following similar derivation presented in Chapter 3, the electric field distribution may be expressed as

$$
E=\left\{\begin{array}{cl}
0 & \left(0 \leq r<R_{a}\right) \\
\frac{U_{0} r_{b}}{e_{b} \lambda r} & \left(R_{a} \leq r<R_{b}\right) \\
\frac{U_{0} r_{b}}{\epsilon_{k} \lambda r} & \left(R_{b} \leq r<R_{h}\right) \\
\frac{U_{0} r_{b}}{e_{m} \lambda r} & \left(R_{h} \leq r<R_{c}\right) \\
0 & \left(r \geq R_{c}\right)
\end{array}\right.
$$

where

$$
\lambda=\frac{1}{e_{b}} \ln \frac{R_{b}}{R_{a}}+\frac{1}{e_{h}} \ln \frac{R_{h}}{R_{b}}+\frac{1}{e_{m}} \ln \frac{R_{c}}{R_{k}}
$$

the other symbols are as defined before.

We may see from Eq.(5.3) and Eq.(5.4) that the electric field intensity will be affected by the permittivity of the intervening material, $\epsilon_{\mathrm{h}}$, and the radius of the hole $R_{h}$. If the permittivity of the filling $\epsilon_{\mathrm{h}}$ is different to that of the medium, $\epsilon_{\mathrm{m}}$ the electric field intensity distribution is interrupted at the interface of two media. For the case of air-dry hole, the intervening medium is the air. The permittivity of air is approximately 


$$
\epsilon_{\mathrm{h}}=\epsilon_{\mathrm{arr}}=\epsilon_{\mathrm{o}}=8.85 \times 10^{-12} \mathrm{~F} / \mathrm{m}
$$

If we assume the electric field in the clay-water-electrolyte system is established in water-electrolyte and not appreciably affected by the clay particles, we have $\epsilon_{\mathrm{m}}=$ $\epsilon_{\text {woter }}$. From Eq.(5.3), at the hole-soil interface, the radius is $r=R_{t b}$. The field intensity at the inside interface is

$$
E=\frac{U_{0} r_{0}}{e_{0} \lambda_{\text {air }} R_{h}}
$$

where $A_{\mathrm{mir}}$ is determined by Eq.(5.4) with $\epsilon_{\mathrm{m}}=\epsilon_{\mathrm{air}}$. At the vicinity of the outside interface, i.e., at $r=R_{k}$, the field intensity is

$$
E=\frac{U_{0} r_{0}}{\epsilon_{w} \lambda_{\text {air }} R_{h}}
$$

We may see that the field intensity is discontinuous at the interface $r=R_{b}$ since $\epsilon_{\mathbf{m}} \neq \epsilon_{\boldsymbol{\sigma}}$

If the hole is filled with an aqueous clay slurry as the intervening medium, again by assuming the electric field is established in the water-electrolyte, we have : $\epsilon_{\mathrm{w}}=\epsilon_{\mathrm{m}}=\epsilon_{\mathrm{w}}$ (permittivity of water). Thus at the hole-water interface the field intensity in both sides of the interface is equal to

$$
E=\frac{U_{0} r_{e}}{\epsilon_{w} \lambda_{w} R_{h}}
$$

where $\lambda_{w}$ is determined by Eq.(5.4). Therefore the field intensity remains 
continuous at the interface.

Fig. 5.8 presents the distribution of electric field intensity along radial direction in a perfect cylindrical geometry. The curves represent the distributions in the soil mass with the intervening materials of air and aqueous clay slurry, respectively. The curves are computed based on Eq.(5.3) and Eq.(5.4) with applied voltage $U_{0}=18 \mathrm{kV}$.

It is of particular importance to note the distribution of electric field in the soil mass. As described in Eq.(2.8), the dielectrophoretic force imposed on clay particles is proportional to the square of the electric field intensity. In order to obtain better efficiency in a dielectrophoresis action, we hope to achieve the highest electric field intensity for a given voltage application. As seen in Fig.5.8 that in the air-dry hole, the electric field intensity is concentrated inside the hole attributed to the high permittivity of pore water which prevents the electric field from propagating in the soil mass. The net effect is the electric field intensity in the soil mass is largely reduced. When the hole is filled with a clay slurry, the distribution of electric field intensity is continuous throughout, as shown in the solid line in Fig.5.8. Thus a continuous non-uniform electric field distribution is achieved, which is more favourable for the action of dielectrophoresis. It may be seen that in the later case, the electric field intensity is significantly higher in the soil, which would impose the larger dielectrophoretic force on clay particles. As a result, one may expect that dielectrophoresis would be more effective when an aqueous slurry is filled in the hole.

The results from the Gloucester clay tests confirmed the theory. We may see from Table 5.1 that for soil samples with similar water content, better effectiveness 
is obtained in the samples with slurry filled holes than that with the "dry" hole. The effect of electric field intensity may be illustrated more clearly when we compare the results of tests with different intervening media. As shown in Fig. 5.6, the sample with a "dry" hole reflect the lowest effectiveness in terms of decrease of water content, which can be interpreted in terms of the distribution of electric field intensity as discussed before.

On the other hand, the case of water as the intervening medium leading to low effectiveness, as shown in Fig. 5.6, which may be attributed to two factors:

1. Because of the relatively large amount of water in the hole, the exhaustion process by the electric field, including vibration generated by the alternating field and heating, is prolonged;

2. The system will return to the state of "dry" hole when the intervening water is exhausted, causing the drop of electric field intensity in the soil mass.

The distribution of electric field intensity is of particular importance in dielectrophoretic process in clays. Once a non-uniform electric field is applied, the clay particles in the system are acted upon the dielectrophoretic force, which is determined by the electric field intensity, the size of the particles and the polarizability of the clay, Eq.(2.8). The first reaction is that the clay particles tend to move towards the direction of lower field intensity because of the negative polarizability. In a cylindrical electric field geometry, the tendency of motion is outward from the central electrode. The clay at vicinity of the central electrode experience larger force than those at outer ground electrode because of the higher field intensity at the central region. Consequently, the pore water in the central 
region enters the central hole and evaporates rapidly because of exhaustion of intensive electric field in the region near the electrode. As the process continues, the water content in the central region reduces. The overall effect is a drying process starting from the central area and propagating progressively outwards along the radial direction. The similar observations were also described by Lockhart (1983) and Ho (1990). Lockhart noted the "conditioning effect" in his test series, i.e., the effect of dielectrophoresis was observed after a time period from the moment of electric field application. Ho described in his model test that the soil was actually turn into a rock under excessive high voltage for a relatively long period of time.

\subsection{Effect of Polarizability}

We know that once the electric field geometry and intensity are determined, the dielectrophoretic force imposed on clay particles is solely dependent on the polarizability of a clay-water-electrolyte system.

It has been shown in Chapter 3 that the apparent permittivity is affected by the water content and clay minerals of the clay-water-electrolyte systems, Fig. 3.9. However, the effective polarizability is not affected by these factors attributed to the conductive regime where the systems are located. As shown in Table 4.4, the effective polarizabilities remained unchanged upon disturbance in the two Leda clays, indicating that the dielectrophoretic force will be constant regardless the disturbance status of the soils. On the riher hand, the effective polarizability will increase when the pore fluid salinity increases associated with exhaustion of water in the clay slurry 
as shown in Fig.3.11, resulting the increasing dielectrophoretic force during the treatment process.

The better effectiveness of dielectrophoresis in a "thick" clay slurry filled hole may be interpreted based on the discussion above. The effects of water content of the slurry may be considered in two aspects:

(1). Physical effect. A clay slurry with lower water content has a lower water volume fraction than one with higher water content. Thus during treatment, the energy required to remove the water in the slurry is less, which means the better "dewatering" effect for the same time period. Another contributing factor is that a thick slurry will leave less dry space in the hole after dry-out by exhaustion, thus the drop of electric field intensity across the hole will be less.

(2). Dielectric effect. As the process continues, the water in the slurry reduces and the electrolyte concentration increases, thus the effective polarizability of the slurry increases until it reaches a constant value, as demonstrated in F! ..11. The significance of this effect is that it indicates the dielectrophoresis provess is progressively more effective.

\subsection{Summary and Conclusions}

The effects of electric field intensity and soil system polarizability on dielectrophoresis treatment are discussed in this chapter. The experimental results on the Gloucester clay samples have provided important facts to support the theory. (1). The shear strength of the natural Leda clay recovered in Gloucester was increased significantly by dielectrophoretic treatment under the voltage of $18 \mathrm{kV}$ for 
21 days. The increase of shear strength was associated with the decrease of soil water content;

(2). The efficiency of dielectrophoresis treatment was significantly improved by using a clay slurry as the intervening medium between the electrode and the soil. Furthermore, the effectiveniess of treatment was inversely proportional to the water content of slurry in the hole;

(3). The fact that the effectiveness of dielectrophoresis is dependent on the intervening media may be attributed to the distribution of electric field intensity in a cylinariciu field geumerry

(4). The dielectrophoresis in ciays is a progressive process, i.e., the efficiency of action will increase with the treatment time, which may be interpreted by the effective polarizability in relation to the electrolyte concentration in the intervening media.

In conclusion, for the best effectiveness in strengthening a soft clay, we should minimize the space between the electrode and the soil, and fill the space with a "thick" clay slurry to ensure that a continuous electric field is generated. 


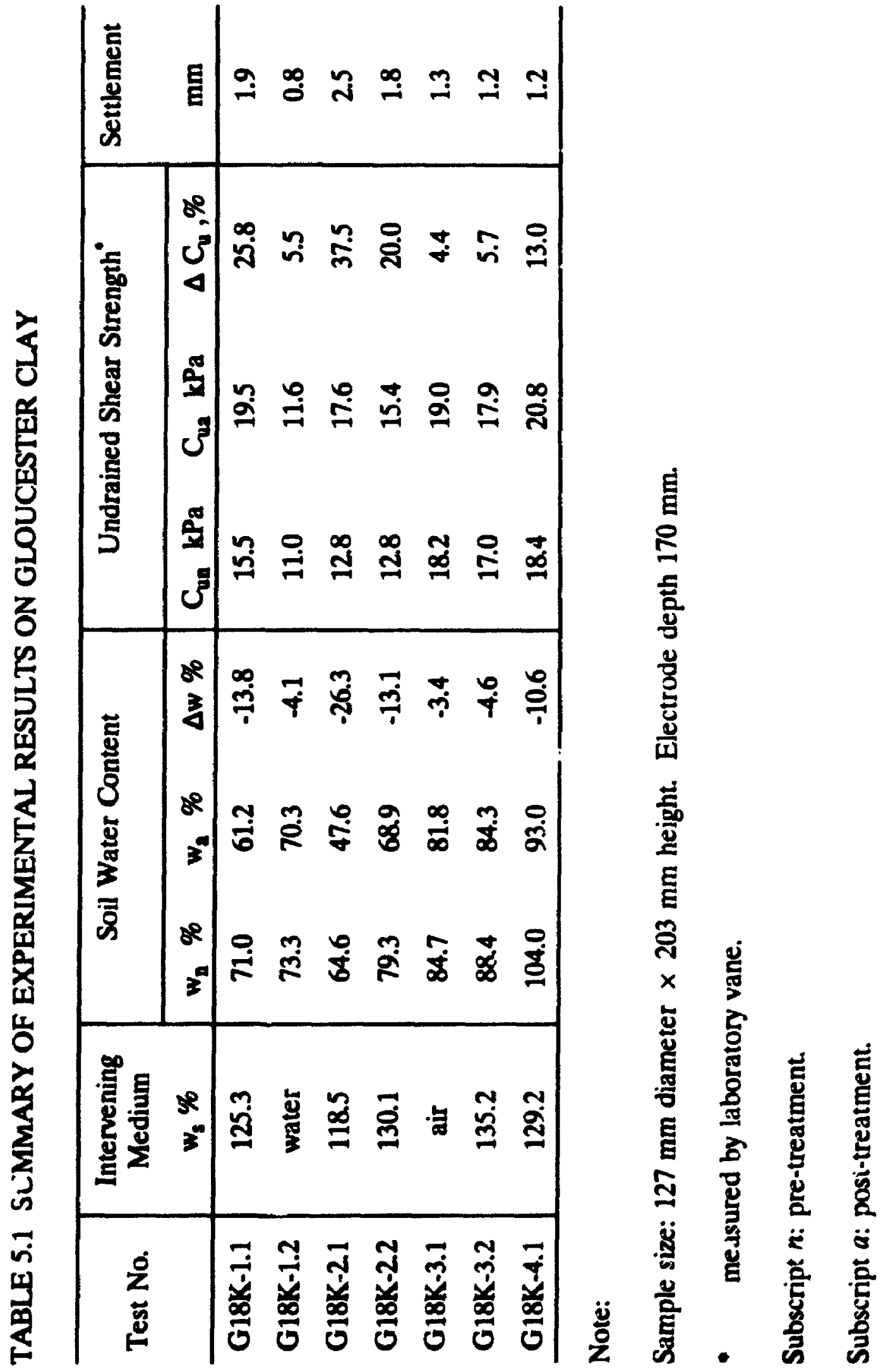




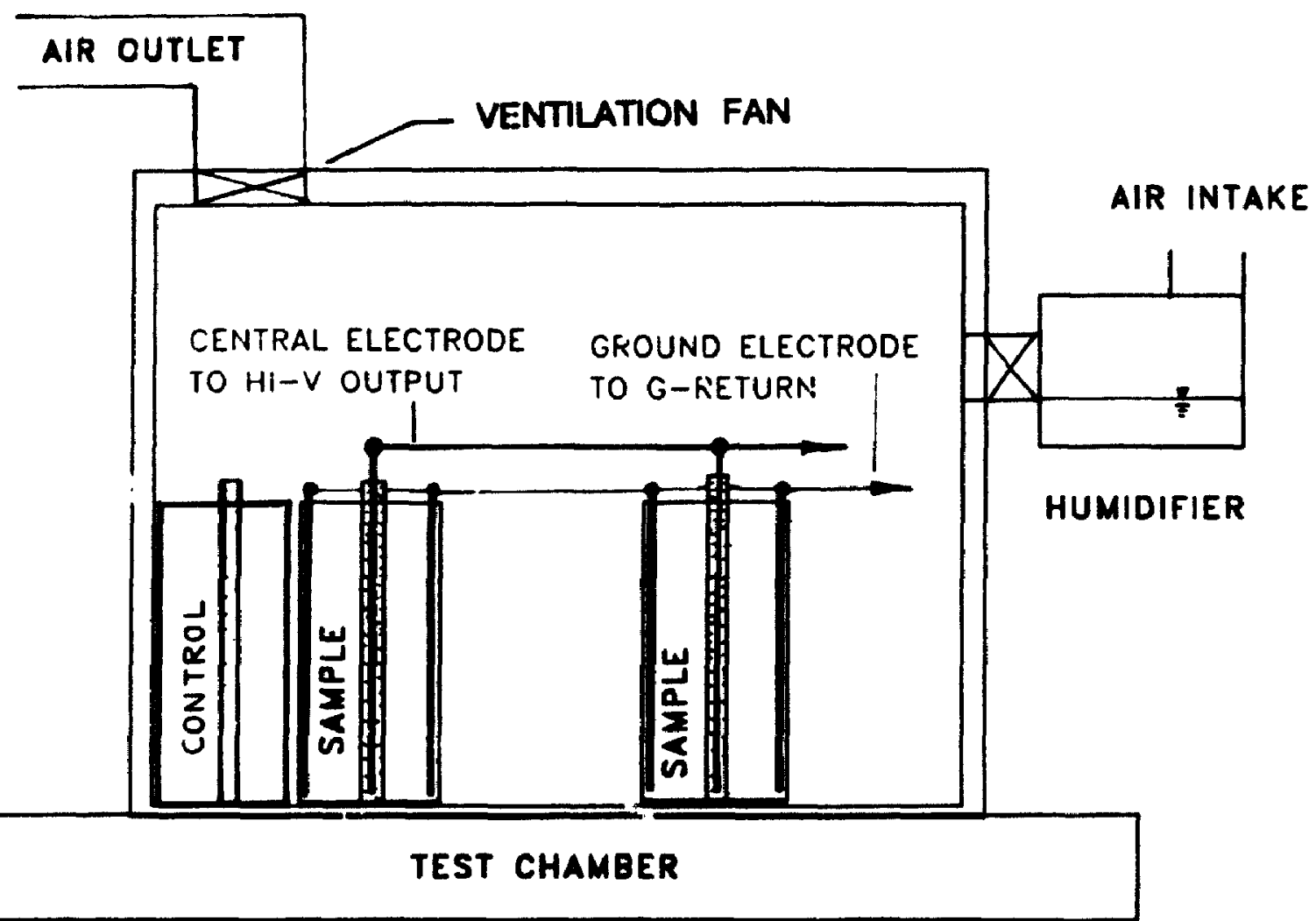

SIGNAL GENERATOR

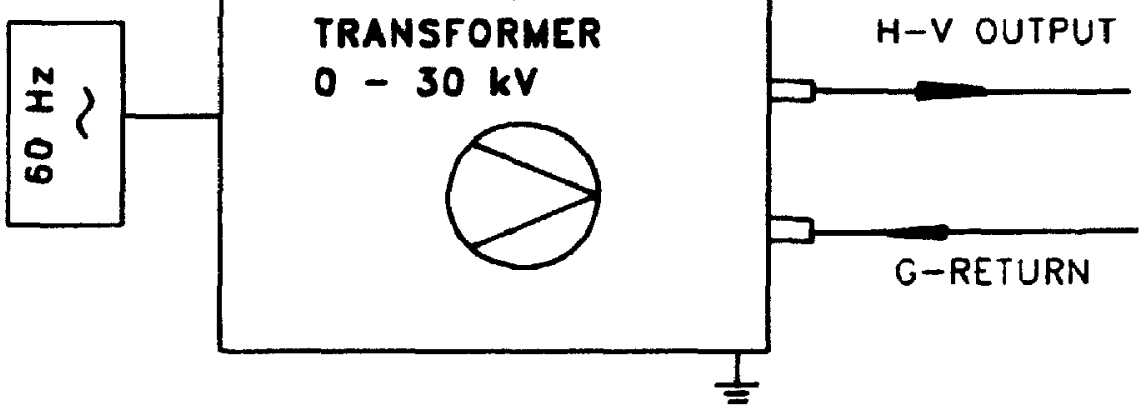

FIGURE 5.1 TEST APPARATUS 


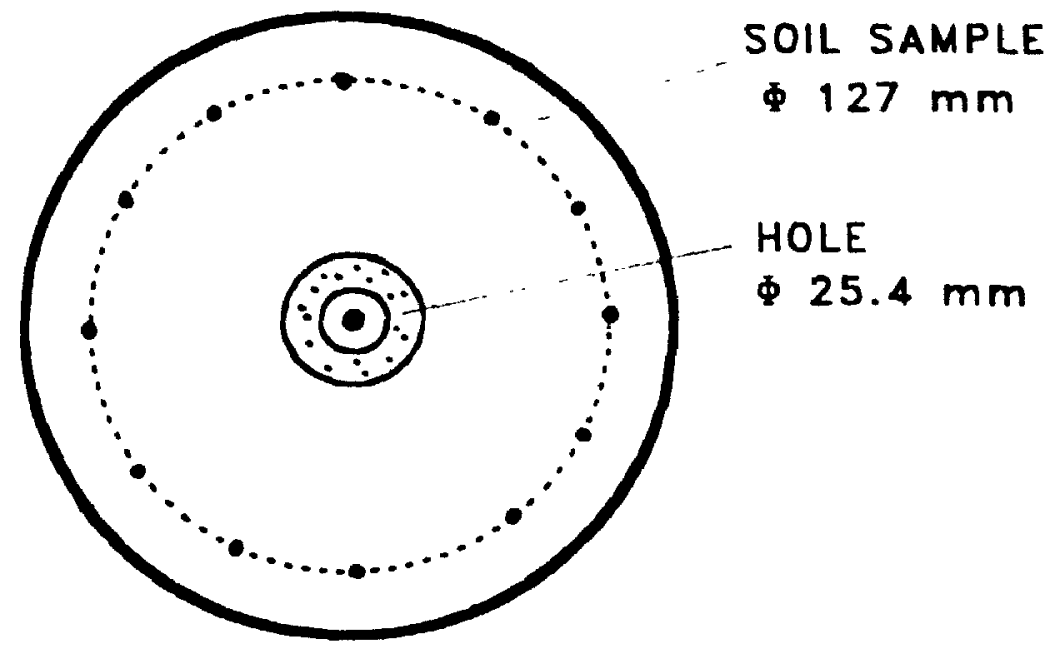

A.) TOP VIEW

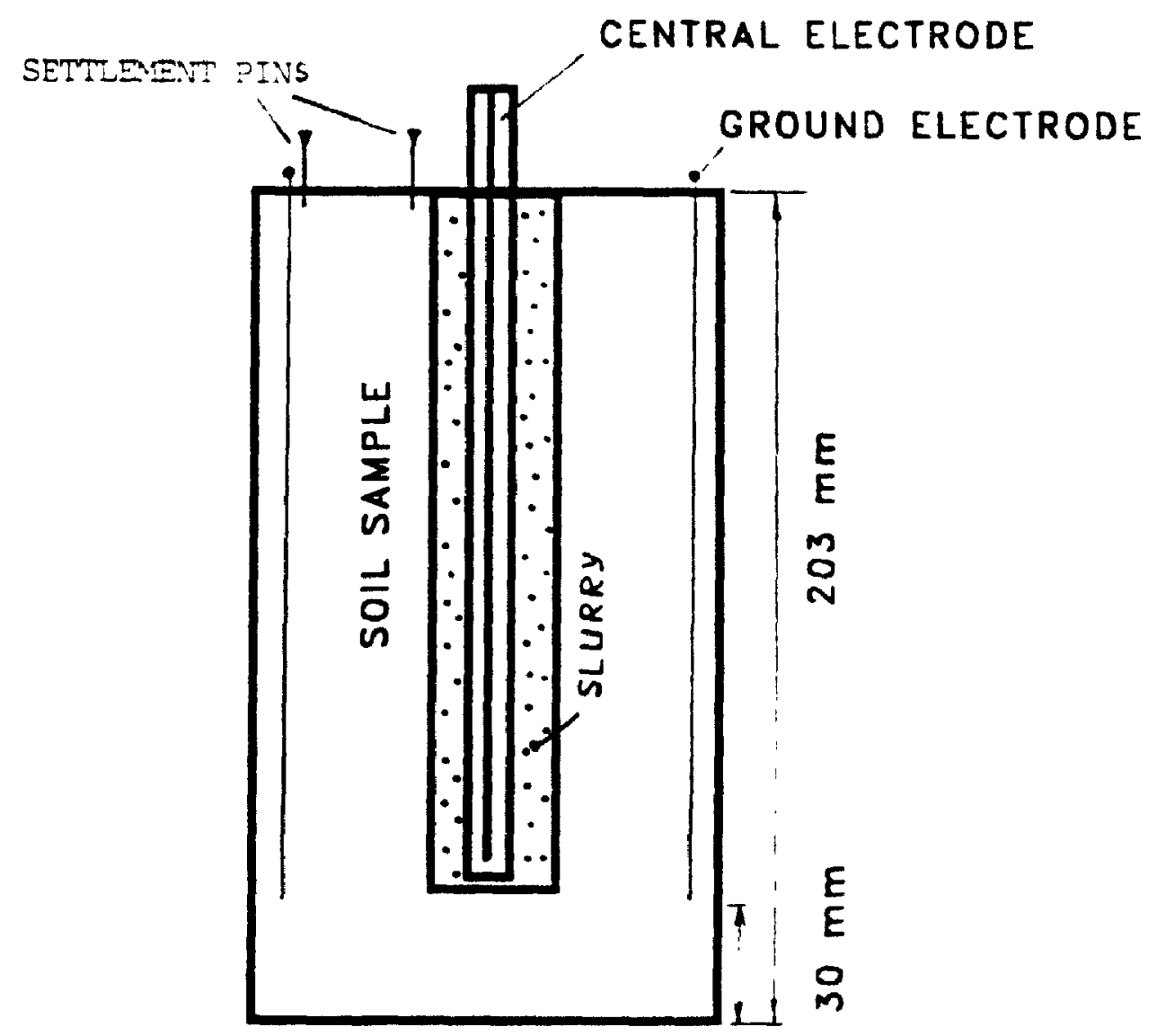

B) SECTION VIEW 


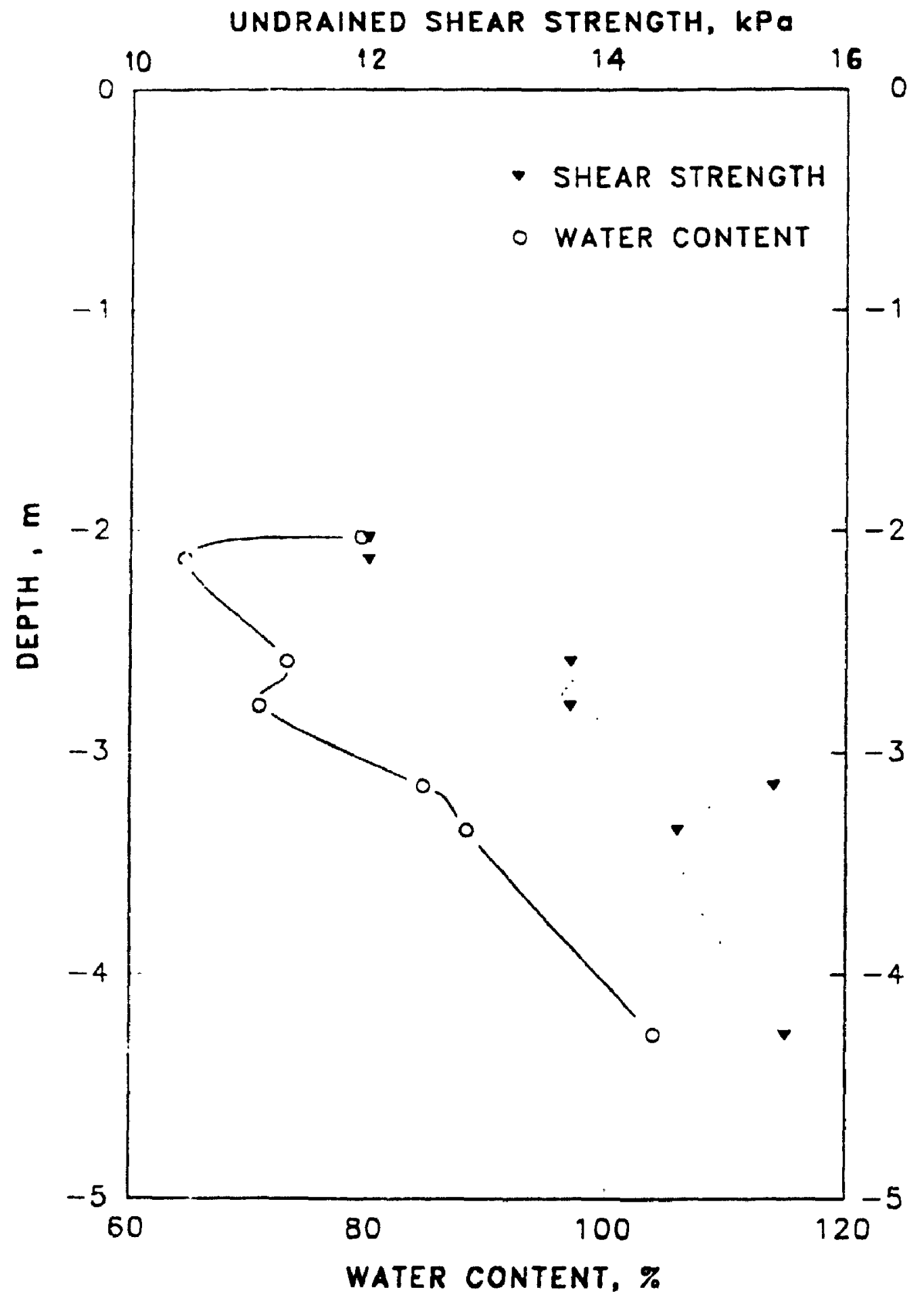

FIGURE 5.3 PROFILE OF GLOUCESTER CLAY AT TEST LOCATION 


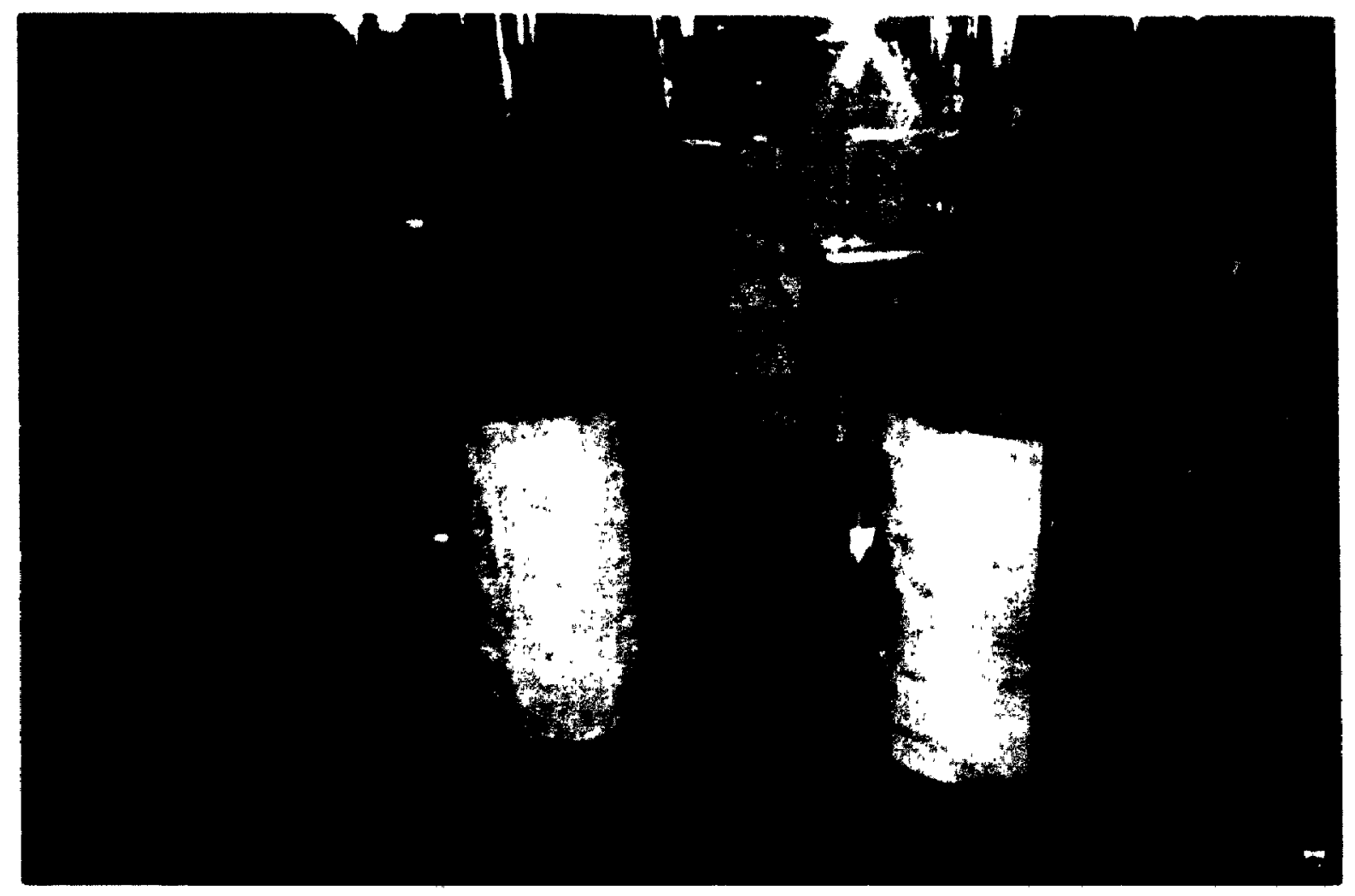

FIGURE 5.4 TEST-SET-UP: DEP TEST ON GLOUCESTER CIAY 


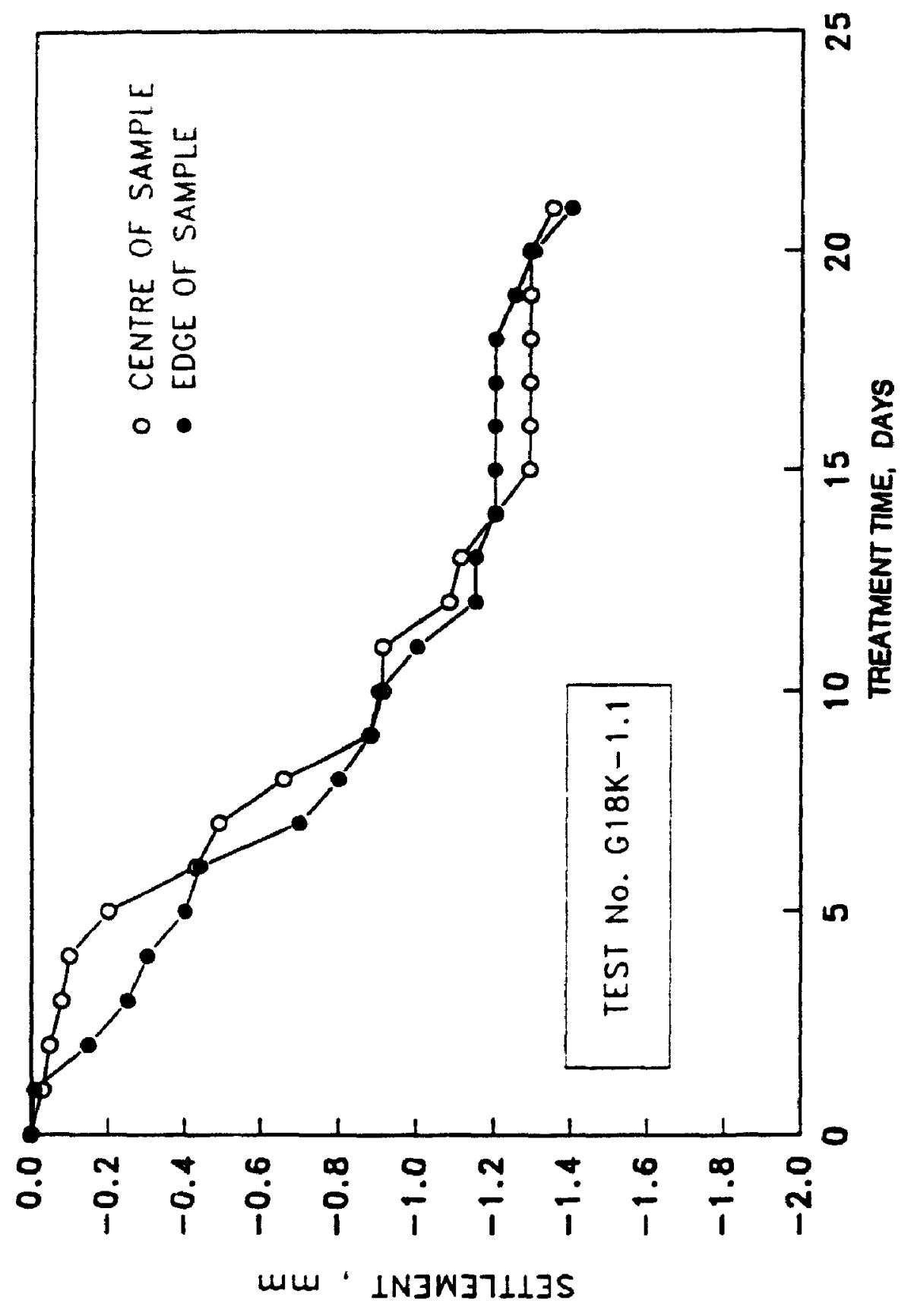

焉 


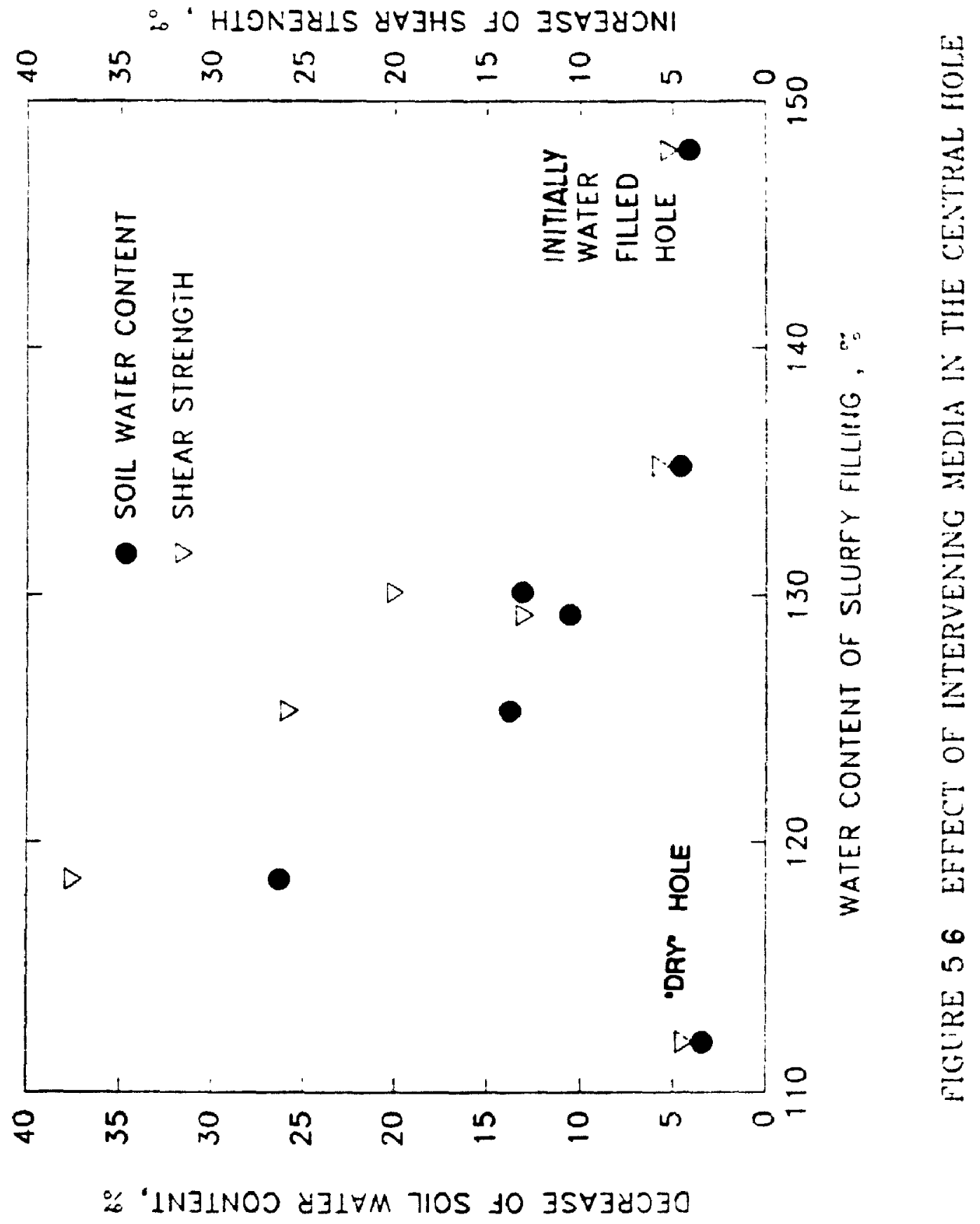




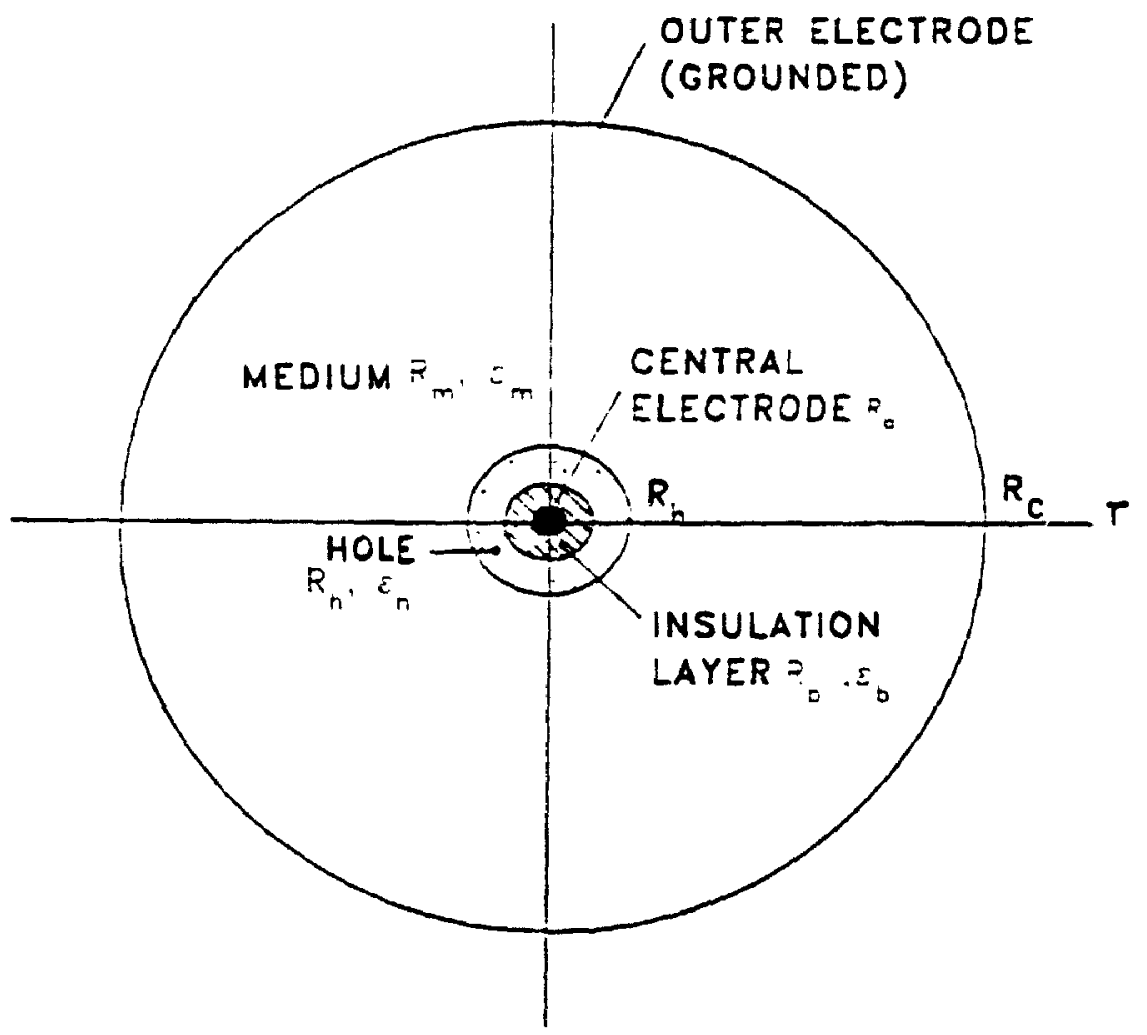

FIGURE 5.7 NON-LNIFORM ELECIRIC FIELD WITH CILINDRICAL SYMMRY 


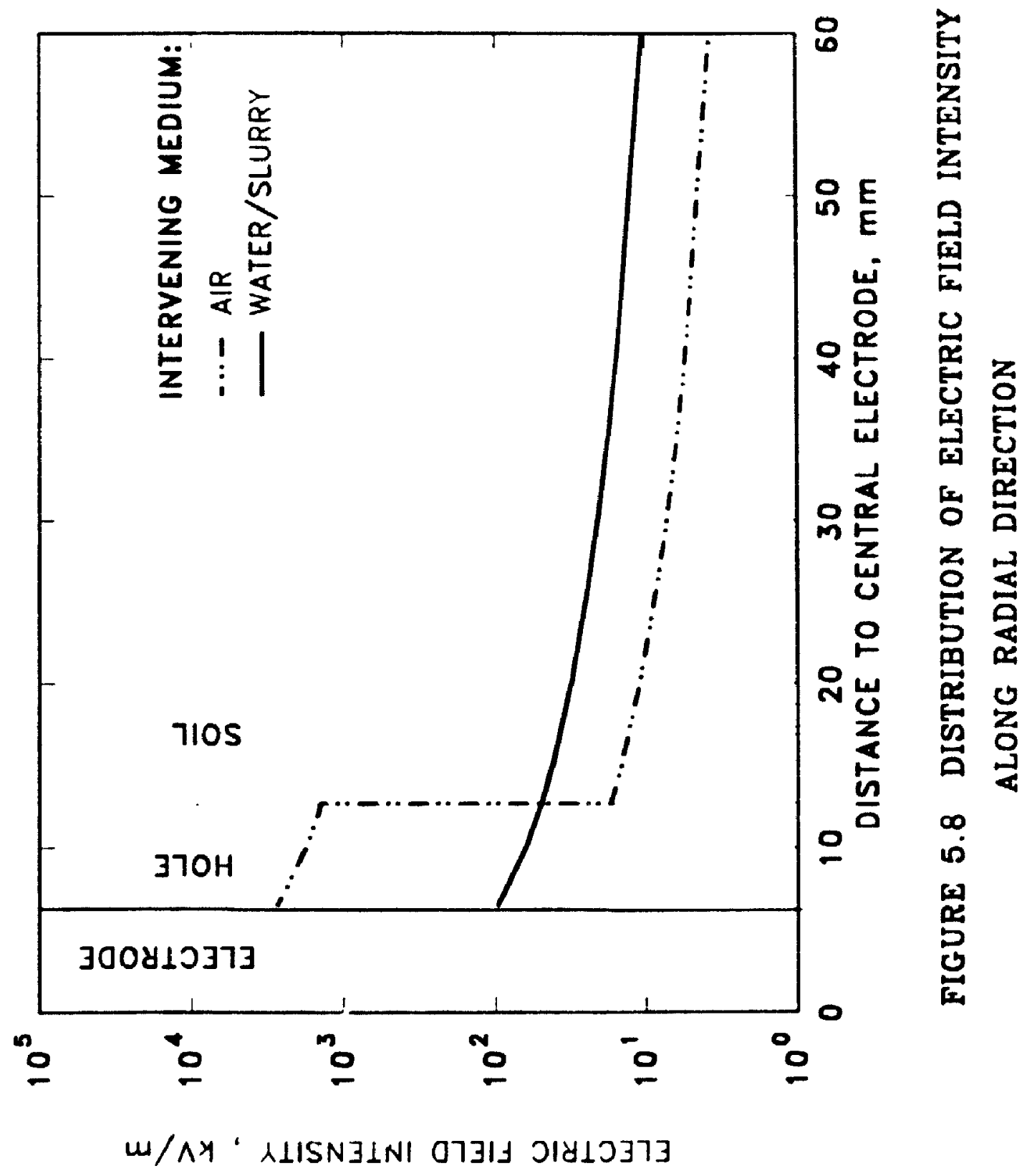




\section{CHAPTER 6}

\section{DIELECTROPHORETIC EXPERIMENTS ON ORLEANS CLAY}

\subsection{Introduction}

The experimental study on the Gloucester clay as discussed in Chapter 5 has confirmed the effectiveness of dielectrophoresis on strengthening soft clays. In the test series on the Gloucester clay tube samples, the electric field configuration had nearly perfect cylindrical symmetry, which is ideal for parameter studies, such as the applied voltage and the intervening media in the central holes, as discussed in the previous chapters. However, the distribution of soil properties after treatment in relation to the distribution of electric field intensity could not been determined in the test series due to small sample size. Furthermore, the practical electric field configurations for engineering applications, such as the electrode-pair and the electrode-matrix, should be investigated in comparison to the theoretical analysis.

Based on the results of previous study on the Gloucester clay, an experimental program on the block samples of the Orleans clay was initiated. The post-treatment distributions of water content and shear strength of the soil in relation to the electric field intensity was investigated. The changes in physical, mechanical and chemical properties may provide important information with respect to the interparticle actions in natural clays. The understanding obtained through the investigation may be eventually beneficial for engineering practice.

This chapter presents the design, procedure and results of the experimental program. The experimental data are used to compare with the distributions of 
electric field intensity as derived from the theoretical analyses.

\subsection{Experimental Design and Procedure}

The design of the experiment series in the Orleans clay block samples was based on the considerations that the effects of natural drying and exhaustion at the high-voltage electrode should be eliminated or compensated. Thus the relationship between the changes of soil properties and the electric field intensity may be determined.

\subsubsection{Soil Stratigraphy}

The properties of the Orleans clay has been described in Chapter 4. The block samples, $30 \mathrm{~cm}$ cube in size, were excavated from depth $4.5 \mathrm{~m}$ to $5.1 \mathrm{~m}$. The water contents of the soil were tested at intervals of about $10 \mathrm{~cm}$ along the sampling zone, with a profile shown in Fig.6.1. The soil may be divided into two layers in terms of the properties for detailed analyses: the Layer I (upper layer) has the water content in the range of $71.77 \%$ and the Layer II (lower layer) in the range of $65-69 \%$, respectively. The Atterberg limits corresponding to each layer are also presented in Fig.6.1. It may be seen that the two layers have similar plastic limits, i.e., PL $=31$ for the Layer $I$ and $P L=32$ for the Layer II, respectively. However, the liquid limits are slightly different, namely, $L L=70$ for the Layer I and $\mathrm{LL}=63$ for the Layer II. The water contents are slightly higher than the liquid limits, indicating the sensitive nature of the soil.

The undrained shear strength was determined by unconfined compression 
tests. The stain rate used was $1 \%$ per minute in all tests. The typical relationship of the shear stress verses the vertical strain in the unconfined compression tests is presented in Fig.6.2. It may be seen that the soil exhibits brittle behaviour, with the failure strain ranged between $0.7 \%$ to $1.1 \%$ for most samples and significant drops of shear stress after failure. The sensitivity of the soil was determined from the unconfined compression tests on the remoulded samples, estimated at $40-60$, Table 4.1. The average shear strengths are $c_{u}=68.8 \mathrm{kPa}$ for the Layer I and $c_{u}=61.7 \mathrm{kPa}$ for the Layer II.

\subsubsection{Test Preparation}

The soil block sample with completed test preparation was shown in Fig.6.3 and the photograph in Fig.6.4. Prior to a test, a square of the wax cover on the soil block, approximate $2 \times 2 \mathrm{~cm}$ in size, was cut off for the installation of the highvoltage electrode. A hole of $16 \mathrm{~mm}$ diameter and $250 \mathrm{~mm}$ depth was carefully drilled by means of a drill machine to minimize disturbance to the soil. The hole was then filled with a slurry made of the extruded soil from drilling. The high-voltage electrode composed of a copper bar and a silicon oil insulated shell, as shown in Fig.28, was inserted into the hole. A $10 \times 10 \mathrm{~cm}$ plexiglas plate was placed above the hole to support the electrode. The depth of the electrode may be adjusted by an electrode holder, as shown in Fig.6.3. The plate was also served as a window for observation during testing period.

To install the ground electrode, a hole of $10 \mathrm{~mm}$ diameter and $250 \mathrm{~mm}$ length was drilled. The ground electrode was composed of a copper bar, $4.8 \mathrm{~mm}$ in 
diameter, inserted tightly in a flexible PVC tube. The outside diameter of the PVC tube was $7 \mathrm{~mm}$. The tip of the ground electrode was sealed by a PVC cap. The space between the ground electrode and the hole was filled with the clay slurry. It was found that the insulation to the ground electrode would provide better effectiveness and economy in dielectrophoresis treatment as the power dissipation is minimized. Also the insulation to both high-voltage and ground electrodes may define the distribution of electric field more precisely by eliminating the influence of soil conductivity. This is especially important in a relatively conductive soil.

After installation of electrodes, the surface of the soil block was staled by a layer of wax to minimire water evaporation, as shown in Fig.6.3.

The plastic settlement pins were installed on top of the soil block at designated locations, as illustrated in Fig.6.5. Care was taken so that the pins were not in touch with the wax and at the same time, the soil exposure remained minimum.

The soil block was then placed inside the tes. chamber. The central and ground electrodes were connected to the high-voltage and the ground return outlets of the power supply, respectively. The test apparatus has been described in detail in Chapter 5. The applied voltage in this test series was $15 \mathrm{kV}$.

\subsubsection{Test Procedure}

Three ests were conducted under the same applied voltage $(15 \mathrm{kV})$ with different electric field configurations. The distributions of the corresponding electric fields have been derived and illustrated by the equi-intensity lines as seen in 
Fig.3.6 and Fig.3.4. respectively.

(1). Test No.B2: The electrode-matrix as discussed ia Chapter 3, Section 3.2.6. The high-voltage electrode was installed in the centre of Block No.2 with four ground elect:odes at the four corners. as shown in Fig.6.5(A). The equi-intensity lines of the electrode configuration is shown in Fig.3.6;

(2). Test No.B3: The electrode-pair as discussed in Chapter 3, Section 3.2.5. The electrode-pair was installed on the diagonal line of Block No.3, with the separation distance $2 \mathrm{~s}=100 \mathrm{~mm}$, Fig.6.5(B). The equi-intensity lines of the electrode configuration is shown in Fig.3.4(A);

(3). Test No.B4: The electrode-pair mistalled on the diagonal line on Block No.4, similar to test No.B3 but the separation distance was doubled as $2 \mathrm{~s}=200 \mathrm{~mm}$. Fig.6.5(C), representing the equi-intensity lines shown in Fig.3.4(B). Each test lasted 28 days without interruption.

\subsubsection{Observations}

The applied voltage was stable in the test period. The settlement of the soil sample was monitored and recorded every day. At day- 28 of each test, the power was turned oft and the soil sample was removed from the test chamber and inspected immediately. Slight water condensation was observed under the plexiglas window at the high-voltage electrode, suggesting the water evaporation in the region. The same observation was reported by Lo, et al (1992). The sample afier treatment is shown in Fig.6.6. showing that the holes were completelv dry after treatment. This observation was consistent with that on the Gloucester _.ay samples, as discussed in 
Chapter 5.

The soil was carefully inspected after the wax cover was removed. The soil was intact, without visible cracks after tests No.B2 and No.B4. However, cracks were found after test on No.B3, splitting the block into three parts, as illustrated in Fig.6.7. The main crack was formed close to the line jointing the electrode-pair.

\subsubsection{Post-Treatment Tests.}

After treatment, a program of tests was conducted to investigate the changes in the physical, mechanical and chemical properties.

\section{(1). Physical properties}

The water content and the Atterberg limits were measured before and after treatment.

The soil block was divided into nine sub-blocks and waxed individually for later investigation. Before waxing, the water contents were measured at each sub-block, at locations shown in Fig.6.8. The measurement was conducted both in Layer I and Layer II to investigate whether there was difference in behaviour.

The Atterberg limits of the soil after treatment were tested on both Layer I and Layer II, at the sub-block \#5, as illustrated in Fig.6.8, where the effect of dielectrophoresis is relatively intensive for all the electric field configurations.

(2). Mechanical properties

The post-treatment mechanical properties investigated included the undrained shear strength, sensitivity, stress-strain behaviour, consolidation behaviour and effective stress parameters. 
The undrained shear strength was determined from unconfined compression tests. After test No.B2, unconfined compression tests were conducted on samples of $50 \mathrm{~mm}$ in diameter and $100 \mathrm{~mm}$ in height, trimmed from the middle portion of the soil block, involving both layer I and layer II. The location of the samples were adjacent to the central electrode. Three tests were conducted. The field intensity at the test location was the highest in the block.

For Block No.3 and No.4. the specimens for unconfined compression tests were reduced to $35 \mathrm{~mm}$ in diameter and $70 \mathrm{~mm}$ in height so that the shear strength of Layer I and Layer II could be determined separately at each sampling location. Note, however, that the properties of layer I and layer II are not strongly different, as discussed in Section 6.2.1. The sampling locations were carefully designated so that they would cover areas of various electric field intensity.

After Test No.B3, cracks occurred on the soil block which disrupted the sampling locations designated for unconfined compression tests. Therefore the tests had to be conducted at locations where no cracks were found.

The ideal condition for shear strength testing was obtained from Test No.B4. The soil block was intact with no visible cracks. The unconfined compression tests could be managed in pre-determined locations. The block was divided into nine subblocks. Ten locations, as shown in Fig.6.8, were chosen to conduct the unconfined compression tests. Two tests were carried out at each location, at the Layer I and Layer II respectively. Thus the distribution of shear strength for each layer can be determined.

The consolidation tests were conducted on the sub-block \#6, Fig.6.8, in the 
Layer I and Layer II.

The post-treatment effective stress properties were examined through a series of consolidated-isotropic-undrained (CIU) triaxial tests after Test No.B4. The samples of standard size, i.e., $50 \mathrm{~mm}$ in diameter and $100 \mathrm{~mm}$ in length were taken at the location showing in Fig.6.8.

\section{(3). Chemical properties}

The post-treatment chemical properties included carbonate content, salinity, C.E.C., $\mathrm{pH}, \mathrm{Cl}$, and $\mathrm{SO}_{4}{ }^{-2}$. The sample was taken at the close vicinity of high-voltage electrode at the Layer 1 .

\subsubsection{Summary}

The experimental program on the Orleans clay block samples was of essential importance towards fully understanding of the principles of dielectrophoresis. In the test series, every effort was made to ensure the reliability of data. Each soil block was treated as an individual source of information for investigating the effects of nonuniform electric field induced dielectrophoresis in the clay. Because of the relatively large size of the soil block samples, it was possible to conduct tests on the soil water content and the unconfined shear strength at various locations with respect to different intensities and gradients of applied electric field intensity. Thus a systemized analysis can be conducted to investigate the changes of soil properties in relation to the electric field configuration. Furthermore, the theoretical analysis to the dielectrophoretic action on clays may be conducted on the basis of the experimental discoveries. 


\subsection{Results of Tests}

\subsubsection{Change of Soil Water Cuntent}

The distribution of water content after treatment is illustrated by contours of equal water contents, as shown in Figs.6.9, 6.10 and 6.11. The numbers marked on the contour lines are the values of soil water content in percentage. The area having a high density of the contour lines represents a high gradient of water content variation, while the area having a low contour line density represents a relatively uniform water content. The contour maps were prepared using the computer program SURFER (1987 edition).

(1). Test No.B2: Electrode-Matrix

The first test, test No.B2, was conducted under the action of an electrodematrix. The electric field intensity distribution has be derived in Chapter 3, Section 3.2.6. The arrangement of electrodes is illustrated in Fig.6.5(A).

Fig.6.9(A) and Fig.6.9(B) illustrate the distribution of soil water contents in the Layer I and Layer II respectively. It may be observed that the four sides of the block appear to have the lowest water content and the water content was observed slightly higher at the central area of the block.

By comparing with the equi-intensity lines showing in Fig.3.6, it may be suggested that in the test No. B2, the soil water tended to concentrate at the region of higher electric field intensity.

(2). Test No.B3: Electrode-Pair $(s=50 \mathrm{~mm})$

The Test No.B3 was conducted under the action of an electrode-pair, as discussed in Chapter 3, Section 3.2.5. The arrangement of electrodes is illustrated 
in Fig. 6.5 (B), with the electrode separation distance $2 \mathrm{~s}=100 \mathrm{~mm}$.

Fig. 6.10 (A) and Fig. 6.10 (B) illustrate the distribution of soil water contents in the Layer I and Layer II respectively. We may see from the contour maps that the region of higher water content is in the central area. at the vicinity of the electrodes. The results indicate again that the soil water tended to concentrate at both electrodes. There is indication of more concentration at the high-voltage electrode. The gradient of water content variation is slightly higher than that of Test No. B2.

(3). Test No.B4 : Electrode-Pair $(s=100 \mathrm{~mm})$

Comparing to the electrode-matrix, an electrode-pairs may provide larger nonuniformity in terms of electric field intensity so that its effect may be studied in clear detail. Therefore, the electrode-pair configuration was repeated in Test No.B4 with the electrode separation distance $2 \mathrm{~s}=200 \mathrm{~mm}$, as shown in Fig. 6.5 (C).

The distribution of soil water contents after Test No.B4 is presented in Fig.6.11 (A) and Fig. 6.11 (B) for Layer I and Layer II, respectively. It may be seen that the distribution is similar to that of Test No.B3, with the water content higher in the central region.

The factors affecting the soil water content after treatment include:

(A). Addition of clay slurry in the hole for electrode installation, which would increase the soil water content slightly, especially in the vicinity of the hole;

(B). Exhaustion at high-voltage electrode due to the high voltage altemating electric field, which could accelerate the evaporation of soil water;

(C). Possibility of water dissociation caused by the electric field. 
In the test series, the holes for electrode installation were smaller in diameter than those in the Gloucester clay tests and the slurry had lower water content for better effectiveness. As a result, the effect of slurry to the soil water content may be considered negligible. On the other hand, the entire soil block, including the hole for electrode installation, was sealed from outside atmosphere, so that the effect of natural drying was minimized. Hence we may consider that the soil water content after treatment represents the true effect due to the action of dielectrophoresis. It may be seen from Table 6.1 that after treatment, she overall soil water contents were little changed. Therefore, the distribution of soil water content after treatment can be considered as an indication of soil water migration due to the effect of dielectrophoresis. The experimental discoveries may be summarized as the follows: (1). The soil water contents after dielectrophoresis treatment were controlled by the electric field configurations. The soil water content was found relatively high in the vicinity of electrodes, i.e., at the region of high electric field intensity.

(2). The patterns of water content distribution for Layer I and Layer II are consistent, suggesting that the treatment was uniform along the electrode depth.

(3). In general, for the Orleans clay, the overall effect of dielectrophoresis on the soil water content was small. 


\subsubsection{Settlement}

In general, the settlements observed were fairly small. The examination of the results indicated that there was no pattern of distribution radially. Fig.6.12 presents the average settlements of the three soil blocks in relation to the treatment time. It may be seen that the average settlement at the end of treatment ranged fromi 0.2 $\mathrm{mm}$ to $0.8 \mathrm{~mm}$, corresponding to the vertical stain ranged from $0.07 \%$ to $0.3 \%$. The largest average settlement took place after Test No.B4. It is observed from Fig.6.12 that the rate of settlement decreased after about 14 days of treatment.

\subsubsection{Change of Undrained Shear Strength}

The soil shear strength increased significantly after dielectrophoresis treatment. The overall increase ranged from $12.5 \%$ to $44.0 \%$, as seen in Table $6.2(1)$. The highest overall increase was registered in Block No.2 where the electrode-matrix provides more intensive field than the electrode-pairs, as illustrated in Figs.3.4 and 3.6. It should also be noted that in Block No.2, the shear strength was obtained from the samples trimmed in the area adjacent to the central electrode, where the electric field inte:isity was :elatively high.

The overall increase of shear strength in the Block No.3 is the lowest among the three blocks tested. It may be attributed to the sample locations. As mentioned earlier, because of the location of cracks, the shear strength at the vicinity of eiectrodes witl. the highest field intensity could not be tested. The specimens had to be trimmed from the area relatively remote from the electrodes where the electric field intensity was low. 
The shear strength results obtained from the Block No.4 is the average of 10 locations for each layer and therefore, it is considered most reliable and representative for the overall increase of the shear strength. Fig.6.13 shows the contour lines of the shear strength in the soil Block No.4, constituted from the data points as shown in Fig.6.8. The unit of shear strength marked on the contour lines is in $\mathbf{k P a}$ The results from the Layer I and Layer II are presented separately in Fig.6.13(A) and Fig.6.13(B), respectively. We may see that the shear strength is the highest at the surrounding area of the electrodes and the distribution of the shear strength is consistent in Layer I and Layer II.

The distribution of shear strength may be interpreted by means of electric field intensity. By comparing the equi-intensity lines in Fig.3.4(B) and the contour lines in Fig.6.13, it may be seen that the highest increase of shear strength takes place in the region of highest electric field intensity and gradient. The shear strength was less affected at the region of low field intensity and gradient. This confirms the basic principle of dielectrophoresis, i.e., a strong, non-uniform electric field is essential to the effectiveness of treatment.

The shear strength data from Tests No.B2 and No.B3, although not adequate to construct the contour lines, provide supplementary support to above discussion. As described before, the samples taken from the vicinity of central electrode in Block No.2 provided the highest increase of overall shear strength, whereas the samples taken from areas remote to the electrode-pair in Block No.3 provided the lowest increase.

In general, we may conclude that the increase of undrained shear strength was 
evident after dielectrophoresis treatment from all three tests with different arrangement of electrodes for treatment. The efficiency of treatment was related directly with the electric field intensity. More detailed discussion may be found in Section 6.4.

\subsubsection{Stress-Strain Behaviours}

The typical stress-strain relationships obtained from unconfined compression tests are presented in Fig.6.14. For most tests, the strain at failure remained relatively little changed after treatment. The increase of shear strength is significant with a stiffer stress-strain response (i.e., higher undrained modulus), Table 6.2(2). Most soil samples, both for pre-treatment and post-treatment conditions, developed vertical cracks once the maximum shear stress was reached and failed abruptly at post-peak range. The post-peak strengths after treatment were little changed, Table 6.2(3).

\subsubsection{Sensitivity}

The improvement of sensitivity after dielectrophoresis has been reported in previous research (Lo, et al, 1992). The sensitivity after treatment was determined from unconfined compression tests on the same specimens for the undisturbed shear strength. The results are summarized in Table 6.2(4). It is found that the sensitivity of the soil after treatment is reduced $50 \%$ to $70 \%$. 


\subsubsection{CIU Triaxial Tests}

The consolidated-isotropic-undrained triaxial tests were conducted on the soil specimens trimmed from Block No.4 after treatment to investigate the effective stress behaviour. The consolidation pressures used both before and after treatment were 65,125 and $185 \mathrm{kPa}$, in which the first value is approximately the in-situ vertical effective pressure. The strain rate in all tests was $0.02 \%$ per minute. The results before and after treatment are compared in Fig.6.15.

At the in-situ pressure of $65 \mathrm{kPa}$, the shear strength after treatment increases appreciably, showing a peak above the initial stress-strain curve. The post-peak behaviour remains unchanged comparing to that before treatment. The pore water pressire is slightly increased at the peak of the shear stress, and unchanged otherwise, Fig.6.15(A).

The peak of the shear stress after treatment is also observed at the consolidation pressure of $125 \mathrm{kPa}$, as shown in Fig.6.15(B). It is noted that the postpeak strength is increased after treatment. The pore water pressure increases slightly in the stress range between the peak and the post-peak state, and remains unchanged otherwise.

For the tests at the consolidation pressure of $185 \mathrm{kPa}$, the patterns of stressstain behaviour before and after treatment are similar, as seen in Fig.6.15(C). The sample after treatment presents an increase on both shear strength and residual strength. The pore water pressure is also increased slightly in the post-peak state.

The changes in shear behaviour of the clay may be seen more clearly by comparing the stress paths and failure envelopes plotted in a q'-p' diagram as shown 
in Fig.6.16. Because of the increase in preconsolidation pressures after treatment (discussed in a subsequent section), the stress paths after treatment are changed significantly for tests consolidated at $125 \mathrm{kPa}$ and $185 \mathrm{kPa}$. Fig.6.16 also shows that the failure envelope after treatment is significantly higher than the initial envelope. confirming that the strength in terms of effective stresses increased.

\subsubsection{Consolidation Tests}

The consolidation tests were conducted on the Layer I and Layer II of the soil samples. The changes of void ratio versus effective stresses are plotted in Fig.6.17. The results are summarized in Table 6.3.

It may be observed from Fig.6.17 that the consolidation curves in the Layer I and Layer II are very similar, indicating that the treatment is uniform. The percentage of increase is in the range of $35.4 \%$ to $38.6 \%$, as seen in Table 6.3. The increase in preconsolidation pressure provides additional direct evidence that the clay is actually consolidated by the non-uniform electric field. The results are also consistent with the observation made on the shift of effective stress failure envelope discussed in Section 6.3.6.

\subsubsection{Atterberg Limits Tests}

The Atterberg limits tests were conducted on Layer I and Layer II, at the location adjacent to the high-voltage electrode. The results are summarized in Table 6.4. It may be seen that the Atterberg limits had no obvious change after treatment. 


\subsubsection{Chemical Analyses}

The chemical analysis after treatment includes carbonate $\mathrm{CO}_{3}{ }^{=}$, chloride $\mathrm{Cl}$, ulphate $S O:=, p H$, salinity and cation exchange capacity (CEC). The results are summarized in Table 6.5, from which the following observations may be made: (1). Salinity, chloride and sulphate content of the soil water did not indicate significant or constant change.

(2). The pH of the soil water increased slightiy from 8.1 to 8.5 .

(3). The CEC of the soil increased slightly from $16 \mathrm{meq} / 100 \mathrm{~g}$ soil to $19 \mathrm{meq} / 100$ g soil.

(4). The carbonate content increased from $1.1 \%$ to $2.2 \%$, showing a rather significant relative increase.

In general, the chemical properties of the Orleans clay demonstrated very limited change. This is largely attributed to the insulation layer of the electrode, which eliminates the electro-chemical reactions associated with electric current during treatment. The dielectrophoresis is primarily a physical process in which the action of electric field is dominant. The results of chemical analysis will be further discussed in Section 6.4.2.

\subsection{Further Discussion}

\subsubsection{Effects of Electric Field Intensity and Gradient}

The similarity of the soil shear strength distribution, Fig.6.13, and the equiintensity lines of the electric field, Fig.3.4(B), indicates a direct link between dielectrophoresis and soil strength. 
According to th: 'heory, the dielectrophoretic force is proportionill to the gradient of the square of electric field intensity ( $\left(E^{2}\right)$, Eq.(2.S). The relationship min be investigated by plotting the increase of undrained shear sirength aganst the absolute value of $\nabla E^{2}$, as shown in Fig.6.18. The values of $|\nabla E=|$ were calculated by differentiating the square of electric field intensity, Eys. (3.7) to (3.11). An example calculation in ideal cylindrical geometry has been given in Eq.(3.3).

It may be seen from Fig.6.18 that in general, the increase of shear strength follows a linear trend with $\left|\nabla E^{2}\right|$, indicating that the dielectrophoretic force imposed on clay particles is proportional to the increase of soil shear strength. The retore. Whe increase of soil shear strength is controlled by both the intensity and gradic'nt of a non-uniform electric field. In order to obtain better effectiveness in the treattment. the clectric field should be strong and highly non-uniform. 17ig.6.18 also shows that in general, the increase of snear strength was similar in the two layers, which further proves the validity of cylindrical electric field geometry.

The relationship between the electric field intensity ( $E$ ) and the increase of shear strength is plotted in Fig.6.19 for reference. As shown that the datit d'-monstrate a parabolic-like pattern, showing a non-linear trend between the two quantities. It may be seen that the maximum inciease of shear strength of $76 \%$ wass found in Test No.B4 at Layer I, adjacent to the high-voltage elertrode, whereas the shear strength increase was less than $10 \%$ wherever the electric field intensity was lower than $5 \mathrm{kV} / \mathrm{m}$.

\subsubsection{Dissociation of Soil Water}

The chemical analysis results provide some evidence of physico-chemi-ial 
effects of dielectrophoresis on clays. Of the results of chemical analysis, the soil pll. cation exchange capacity (CEC) and carbonate contents have shown slight increase after treatment, as presented in Table 6.5 . These characteristics may be interpreted in terms of dissociaticn of soil water.

The application of a strong, alternating electric ficld on to a clay-waterelectrolyte system provide an energy potential promoting ionic dissociation of water in to $\mathrm{H}^{+}$and $\mathrm{OH}$. The dissociation of water can be confirmed from the chemical data shown as a slight increase of soil $\mathrm{pH}$ after treatment. Table 6.6. resulted from the hydrogen ion adsorption onto clay surfaces. In clay-water-electrolyte systems, the surface potential is controlled by the adsorption of potential determining ions, particularly hydrogen ions and hydroxyl in this case because the concentration of other electrolytes are not changed during treatment. In such systems the charge in the Gouy diffuse layer, $\rho_{2}$, is presented by Eq.(3.20).

If t'.e potential determining ions are $\mathrm{H}^{+}$or $\mathrm{OH}$, the Stern potential, $\mathbf{w}_{\mathrm{d}}$, in Eq.(3.20) is related to $\mathrm{H}^{+}$by a Nernst equation (Sparks, 1986)

$$
\Phi_{d}-\frac{R T}{z F} \ln \frac{H^{*}}{H_{0}^{*}}
$$

or

$$
\Phi_{d}-\frac{R T}{2 F} 2.303\left(p H_{0}-p H\right)
$$

where

$\mathrm{H}^{+}=$Activity of the hydrogen ion;

$H_{o}^{+}=$The hydrogen ion activity when $\Phi_{d}=0$; 
The otner symbols are the same as defined in Chapter 3.

Substituting "1, in Eq.(6.2) into Eq.(3.20), we obtain:

$$
\rho_{2}-\sqrt{\frac{2 c \in R T}{\pi}} \sinh 1.15 z\left(p H_{0}-p H\right)
$$

Eq.(6.3) shows that the soil $\mathrm{pH}$ determines the magnitude of net charge as well as the sign because of its relation to $\mathrm{pH}_{0}$. An slight increase of soil $\mathrm{pH}$ will be associated with a slight increase of the charges in the diffuse layer, observed as the increase of CEC.

Many soils contain free carbonates, iron oxide, alumina, and organic matter. which may precipitate at interparticle contacts and act as cementing agents. Among the cementing agents. carbonates, in the form of calcite $\left(\mathrm{CaCO}_{3}\right)$ and dolomite $\left(\mathrm{CaMg}\left(\mathrm{CO}_{3}\right)_{2}\right)$ are considered as one of the most probable ones (Quigley, 1980).

Soils must be considered as open systems with regard to carbonates. The c(quilibrium reactions relating the various carbonate species in soils are (Linsay, 1979):

$$
\begin{gathered}
\mathrm{CO}_{2}+\mathrm{H}_{2} \mathrm{O} \leftrightarrows \mathrm{H}^{+}+\mathrm{HCO}_{3} \\
\mathrm{HCO}_{3} \leftrightarrows \mathrm{H}^{+}+\mathrm{CO}_{3}=
\end{gathered}
$$

The increase of soil pH. as a result of water dissociation by the electric field, induces the reactions towards the right side of the equations, causing the increase of carbonate content.

It may be seen in Table 6.6 that the carbonate content of the Orleans clay increased to $2.2 \%$ after dielectrophoresis treatment, comparing to $1.1 \%$ before tre:atment. The corresponding reduction of soil sensitivity is in the range of $50 \%$ to 
$70 \%$, Table 6.2 (4). Fig. 6.20 presents the decruase of sensitivity in relation to increase of carbonate cor $3 n t$ after treatment.

The increase of carbonate content in soils would lead to increase of she:ar strength, which has be observed by many authors including Conlon (1966), Kenney (1968), and Townsend et al (1969). It has been further confirmed that the increalse of carbonates would result in the decrease of sensitivity of ledia clay (Yong, et al. 1979). The mechanism involved may be interpreted as that the carbonates function as bonding agents. The carbonates mat clay particles and are themselves so chemically active that they behave like clay mineral thickeners if present in large amounts. In this latter case, it would increase both the undisturbed and remoulded shear strengths and effectively reduce sensitivity (Ouigley, 1980).

\subsubsection{Interparticle Forces.}

The inter-particle forces between clay particles include the repulsive force and attractive force, as defined by van Olphen (1977).

The work performed to bring the particles from infinity to a given distance is the repulsion potential between the particles. The repulsive potential can be plotted as a function of distince, as shown in Fig.6.21(a). We may see that the repulsive potential decreases rapidly with increasing particle separation. The repulsive force is sensitive to the surface properties of clays, such as surface potential, valence, electrolyte concentration and temperature.

The inter-particle attractive forces to compete with the repulsive forces are the general van der Waals force between particles. The distribution of the attractive 
potential is also shown in Fig.6.21(a). We may observe from the distribution of the attractive potential that a slight decrease of particle separation distance will increase the attractive potential substantially. The attractive potential is independent of concentration and valence of the ions, i.e. the attraction remains practically the same when the electrolyte concentration and ion type of the medium are varied.

The summation of repulsion and attraction is constructed by adding the attractive and the repulsive potential at the each particle distance, considering the attractive potential negative and the repulsive potential positive. Fig.6.21 (b) shows the results of these additions. In constructing these net curves, van Olphen (1977) has taken an additional interaction force between the particles into account, namely, a repulsion of a very short range, as shown in Fig.6.21 (a), which probably becomes appreciable at particle separations of the order of $1 \mathrm{~nm}$ or less.

Cementation involves chemical bonding and can be treated as a short-range attraction. Upon the action of dielectrophoresis, the high contact stress induced between particles could extrude adsorbed water and cations and force the mineral surfaces to come close together, reducing the particle separation distance. As shown in Fig.6.21(b), between $d_{1}$ and $d_{2}$ along the $\mathrm{X}$-axis (Particle Separation), a slight reduction of particle separation will induce substantial increase of attractive energy, due to the stiff slope of the interaction energy curve at this portion, which illustrated as a stronger cementation bonding in the soil. The increase of attractive energy may also provide opportunity for cold welding (Mitchell, 1976) between the soil particles as the high contact pressure associated with the increasing carbonates as cementing agents. 


\subsection{Conclusions}

A comprehensive experimental program on the block samples of Orleans clay was conducted. The results showed that:

(1). Dielectrophoresis treatment on the sensitive Leda clay (Orleans clay) may improve soil properties significantly, including the increases of soil shear strength and the pre-consolidation pressure, decrease of the sensitivity, and the increase in shearing resistance in terms of the effective stress behaviours.

(2). It was found that the increase of soil strength was associated with the distribution of the applied electric field. The most significant increase was observed in the area with the highest field intensity and gradient. The latter point was confirmed by the linear relationship between the increase of soil shear strength and the gradient of electric field intensity square $\left(\left|\nabla E^{2}\right|\right)$ which is in turn proportional to the dielectrophoretic force imposed on clay particles. (3). There was evidence that the soil water tended to migrate towards the vicinity of the electrodes.

(4). The change of chemical properties after treatment suggests that the dielectrophoretic process was little affected by the electro-chemical reactions associated with electric current, attributable to the insulation to the electrodes. The strong electric field induced slight changes of some chemical propertics, inchw 'ing CEC, $\mathrm{pH}$ and carbonate content, attributable to dissociation of soil water, which enhanced the effectiveness of treatment, shown as the decrease of sensitivity and increase of cementation bonding.

All of the findings suggests that the effect of dielectrophoresis is significant on the Orleans clay. 


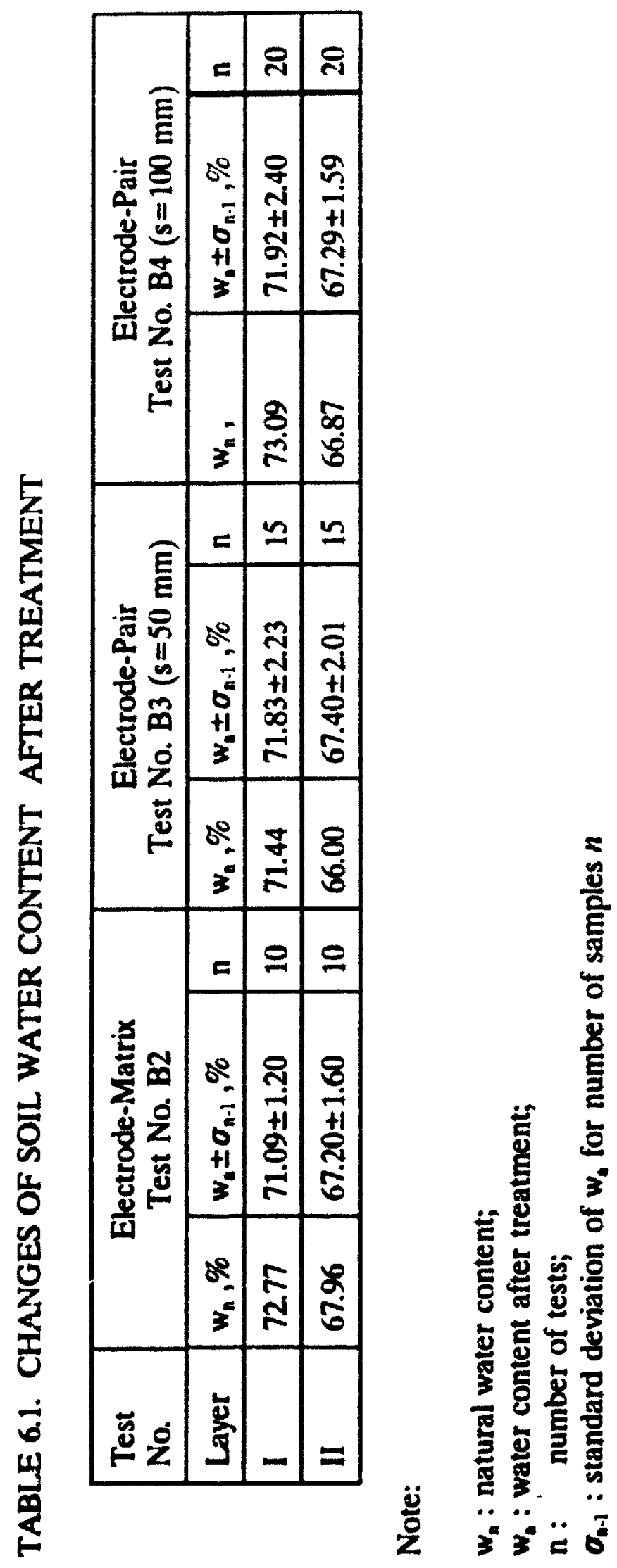


TABLE 6.2. SUMMARIES OF UNCONFINED COMPRESSION TEST RESULTS

(1). Shear Strength

\begin{tabular}{|c|c|c|c|c|c|c|}
\hline \multirow{2}{*}{ Test No } & \multicolumn{3}{|c|}{$\mathrm{c}_{\mathrm{u}}, \mathrm{kPa}$} & \multicolumn{3}{c|}{ Increase of $\mathrm{c}_{\mathrm{u}}, \%$} \\
\cline { 2 - 7 } & Layer I & Layer II & Average & Layer I & Layer II & Average \\
\hline Pre-T & 68.8 & 61.7 & 65.3 & & & \\
\hline B2 & - & - & 94.0 & - & - & 44.0 \\
\hline B3 & 75.9 & 71.0 & 73.5 & 10.3 & 14.9 & 12.5 \\
\hline B4 & 90.9 & 71.8 & 81.4 & 32.1 & 16.2 & 24.6 \\
\hline
\end{tabular}

(2). Undrained Modulus

\begin{tabular}{|c|c|c|c|}
\hline Test No. & \multicolumn{3}{|c|}{ Undrained Modulus, MPa } \\
\hline & Layer I & Layer II & Average \\
\hline Pre-Treatment & 6.17 & 5.41 & 5.79 \\
\hline B2 & - & - & 9.13 \\
\hline B3 & 6.60 & 6.23 & 6.39 \\
\hline B4 & 8.50 & 8.85 & 8.14 \\
\hline
\end{tabular}

(3). Post-peak Strength

\begin{tabular}{|c|c|c|c|}
\hline Test No. & \multicolumn{3}{|c|}{ Post-peak Strength, kPa } \\
\hline & Layer I & Layer II & Average \\
\hline Pre-Treatment & 40.7 & 43.2 & 42.0 \\
\hline B2 & - & - & 41.7 \\
\hline B3 & 39.8 & 37.3 & 38.6 \\
\hline B4 & 39.5 & 35.3 & 37.4 \\
\hline
\end{tabular}


TABLE 6.2. SUMMARIES OF UNCONFINED COMPRESSION TEST RESULTS (continued)

(4). Sensitivity

\begin{tabular}{|c|c|c|c|c|}
\hline Test No. & $\begin{array}{c}\text { Undisturbed } \\
\text { Strength, kPa }\end{array}$ & $\begin{array}{c}\text { Remoulded } \\
\text { Strength, kPa }\end{array}$ & Sensitivity & Decrease, \% \\
\hline Pre-Treatment & 65.3 & 1.3 & 51 & - \\
\hline B2 & 94.0 & 2.8 & 34 & 50 \\
\hline B3 & 73.5 & 2.3 & 32 & 59 \\
\hline B4 & 81.4 & 2.7 & 30 & 70 \\
\hline
\end{tabular}

TABLE 6.3. SUMMARY OF CONSOLIDATION TEST RESULTS

\begin{tabular}{|c|c|c|c|c|c|c|c|}
\hline \multirow{2}{*}{ Test No } & \multicolumn{3}{|c|}{$\mathrm{p}_{\mathrm{c}}, \mathrm{kPa}$} & \multicolumn{3}{c|}{ Incrcase of $\mathrm{p}_{c}, \%$} & \multirow{2}{*}{$\begin{array}{c}\text { OCR } \\
\text { (Average) }\end{array}$} \\
\cline { 2 - 8 } & Layer I & Layer II & Average & Layer I & Layer II & Average & A. \\
\hline Pre-T & 142 & 146 & 144 & - & - & - & 2. \\
\hline B2 & 192 & 198 & 195 & 35.2 & 35.6 & 35.4 & 3.3 \\
\hline B3 & 200 & 199 & 200 & 40.8 & 36.3 & 38.6 & 3.3 \\
\hline B4 & 196 & 200 & 198 & 38.0 & 37.0 & 37.5 & 3.3 \\
\hline
\end{tabular}

Note:

$p_{\varepsilon}: \quad$ pre-consolidation pressure;

OCR: Over-Consolidation Ratio. 
TABLE 6.4. SUMMARY OF ATTERBERG LIMITS TEST RESULTS

\begin{tabular}{|c|c|c|c|c|c|c|}
\hline \multirow{2}{*}{ Test No } & \multicolumn{2}{|c|}{ Liquid Limit , \% } & \multicolumn{2}{c|}{ Plastic Limit , \% } & \multicolumn{2}{c|}{ Plastic Index } \\
\cline { 2 - 7 } & Layer I & Layer II & Layer I & Layer II & Layer I & Layer II \\
\hline Pre-T & 70 & 63 & 39 & 31 & 31 & 32 \\
\hline B2 & 72 & 66 & 34 & 28 & 38 & 38 \\
\hline B3 & 70 & 62 & 33 & 29 & 37 & 33 \\
\hline B4 & 70 & 64 & 35 & 29 & 35 & 35 \\
\hline
\end{tabular}

TABLE 6.5. SUMMARY OF CHEMICAL ANALYSIS RESULTS

\begin{tabular}{|c|c|c|c|c|c|c|}
\hline Test No & $\begin{array}{c}\mathrm{SO}_{4}^{-} \\
(\mathrm{mg} /)\end{array}$ & $\begin{array}{c}\mathrm{CO}_{3}^{-} \\
(\%)\end{array}$ & $\mathrm{pH}$ & $\begin{array}{c}\text { Salinity } \\
(\mathrm{g} / \mathrm{l})\end{array}$ & $\begin{array}{c}\mathrm{C}- \\
(\mathrm{mg} /)\end{array}$ & $\begin{array}{c}\mathrm{CEC} \\
\left(\mathrm{meq} / 100_{\mathrm{g}}\right)\end{array}$ \\
\hline Pre-T & 20 & 1.1 & 8.1 & 0.70 & 225 & 16 \\
\hline B2 & 20 & 21 & 8.3 & 0.88 & 270 & 21 \\
\hline B3 & 14 & 2.3 & 8.5 & 0.70 & 335 & 18 \\
\hline B4 & 45 & 2.2 & 8.5 & 0.85 & 375 & 18 \\
\hline
\end{tabular}




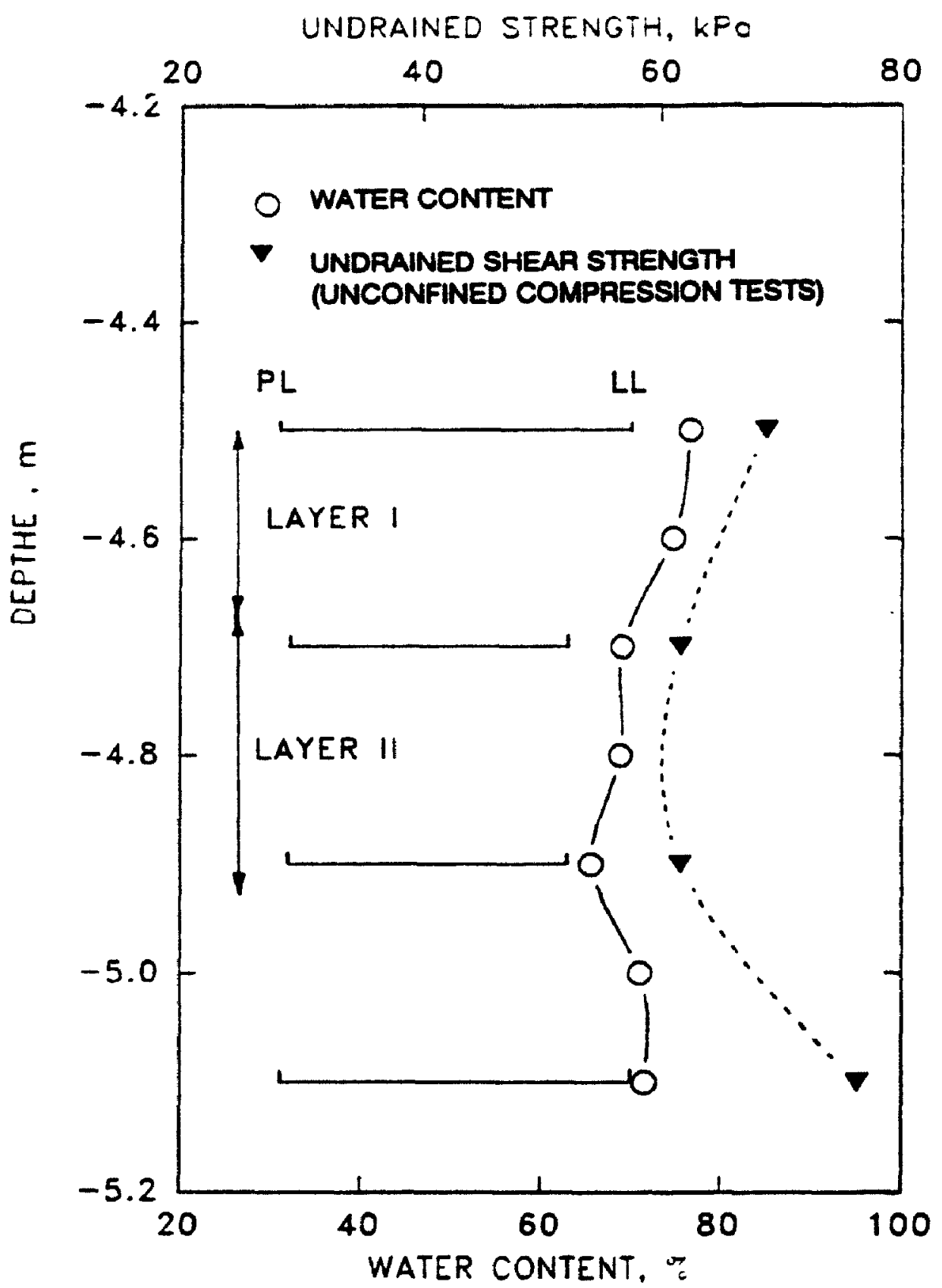

FIGURE 6.1 PROPERTIES OF ORIENAS CLAY 


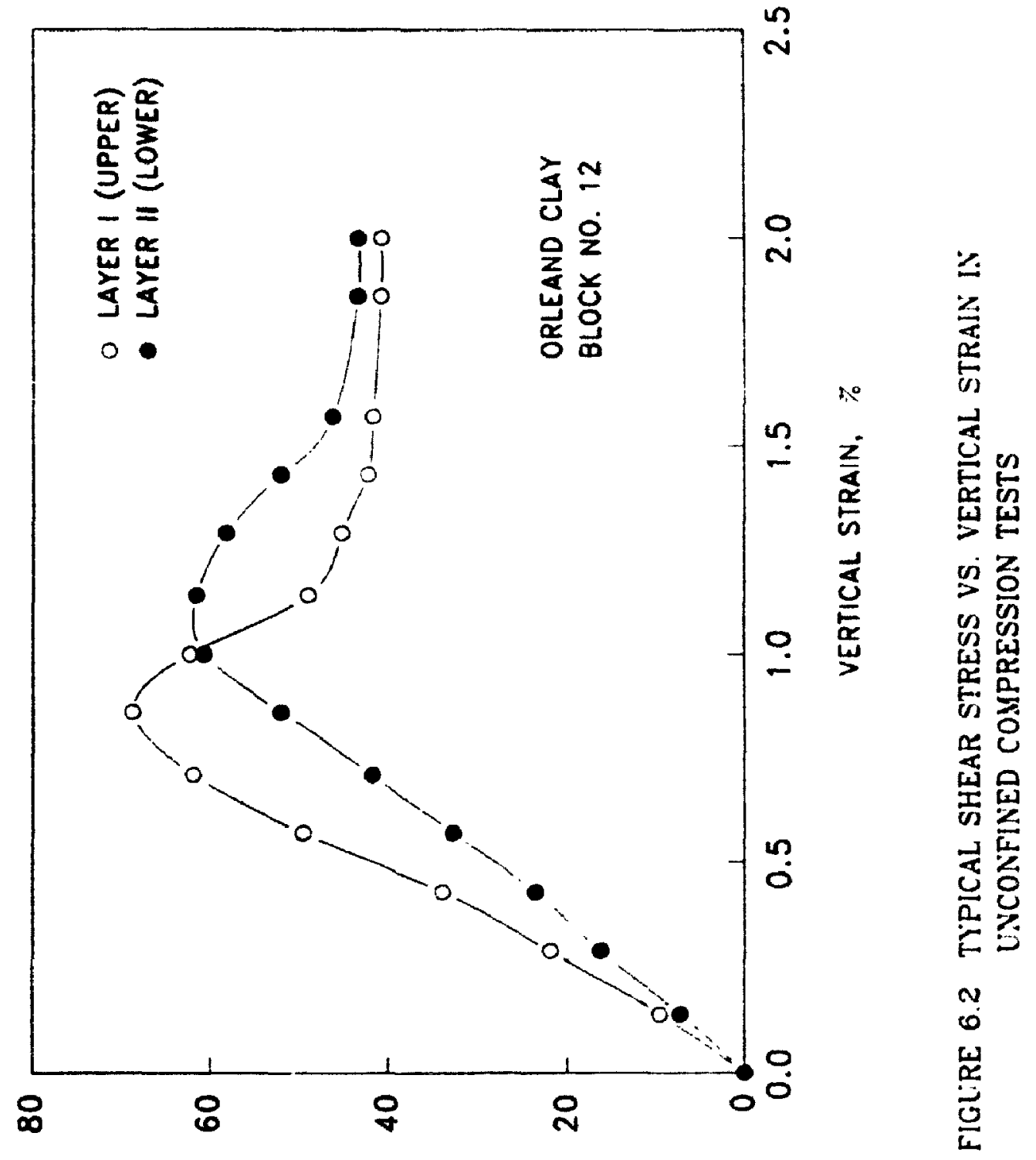

Ddx ' sSzyls arjhs 


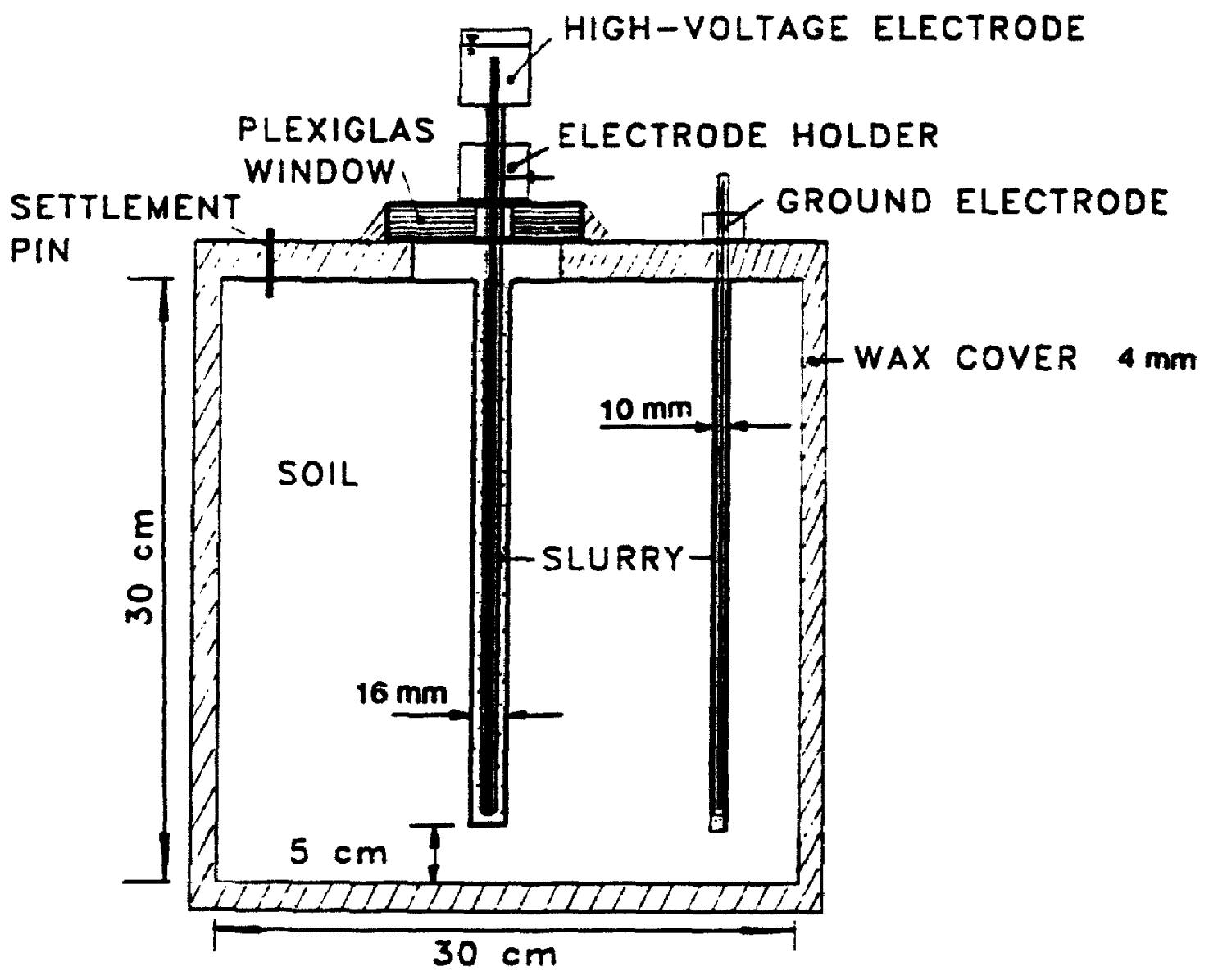

SECTION VIEW 


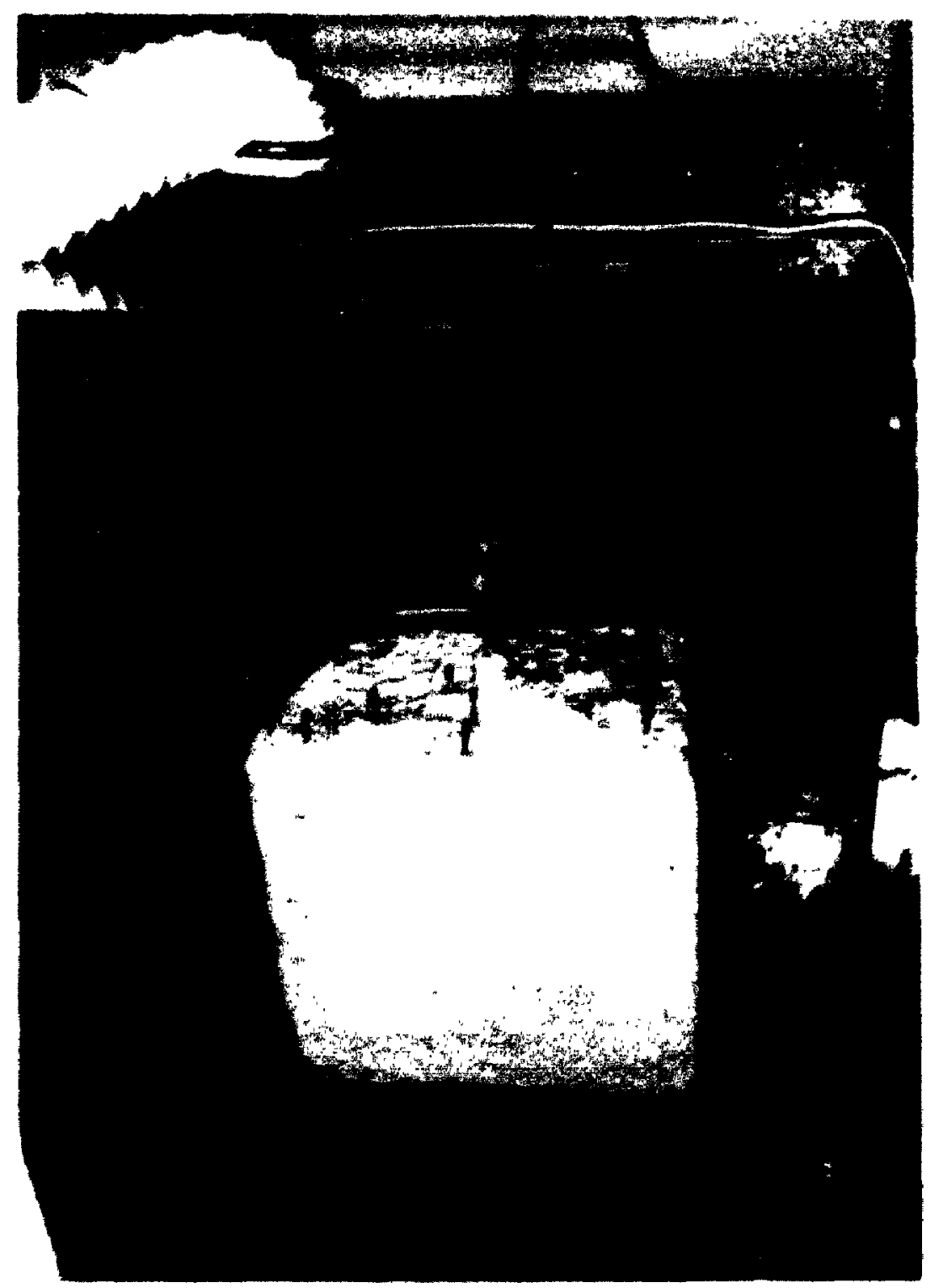




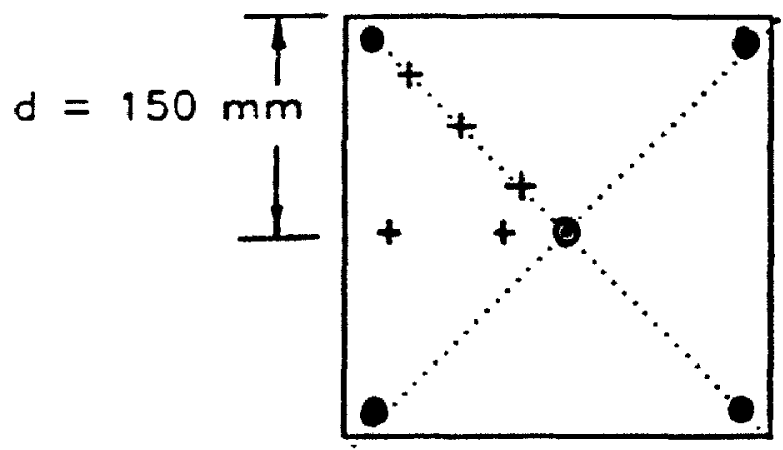

(A) TEST NO. B2

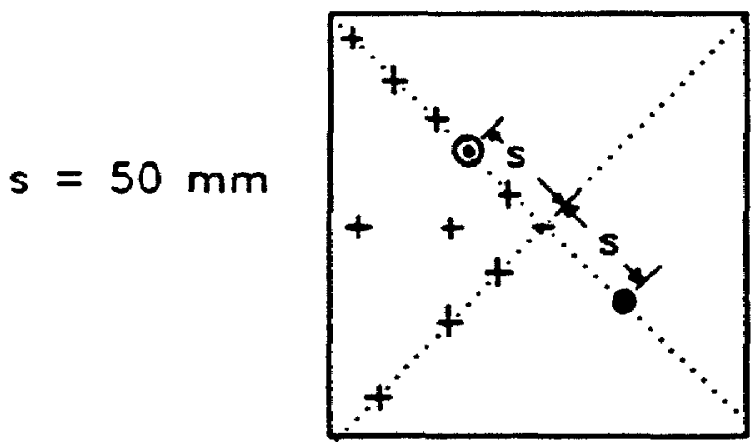

(B) TEST NO. B3

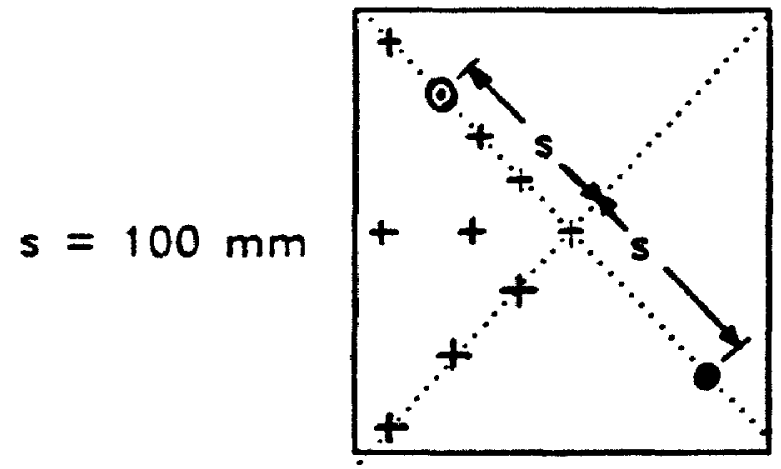

(C) TEST NO. 84

\section{- HI-V ELECTRODE \\ - GROUND E! ECTRODE \\ + SETTLEMENT PIN}




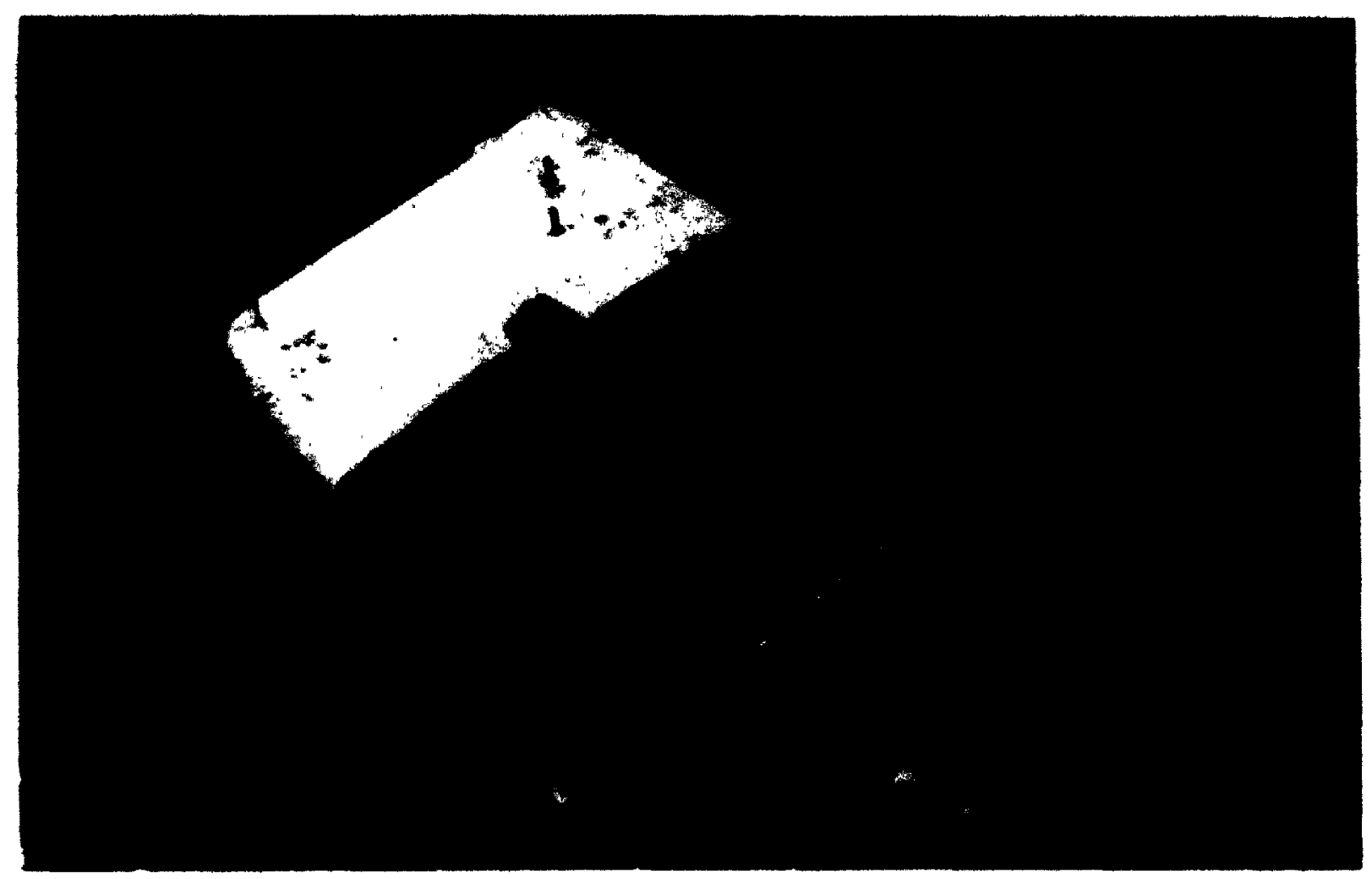

FIGURE 6.6 BLOCK SAMPLE AFTER TREATMENT, TEST NO.B2 


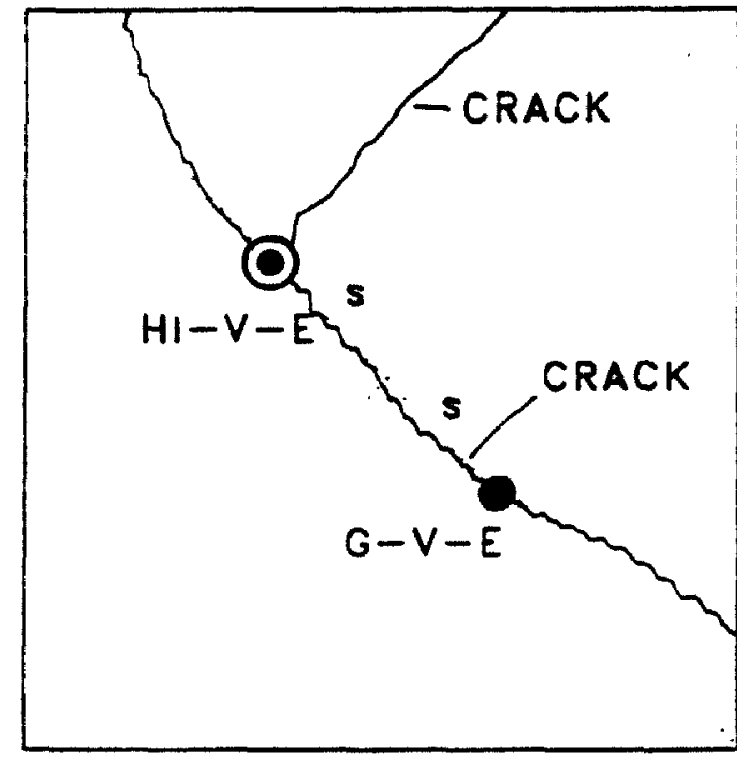

$$
s=50 \mathrm{~mm}
$$

\section{TEST NO.3 BLOCK No.3}

FIGURE 6.T CRACK PATTERN: AFTER TEST NO. B3 


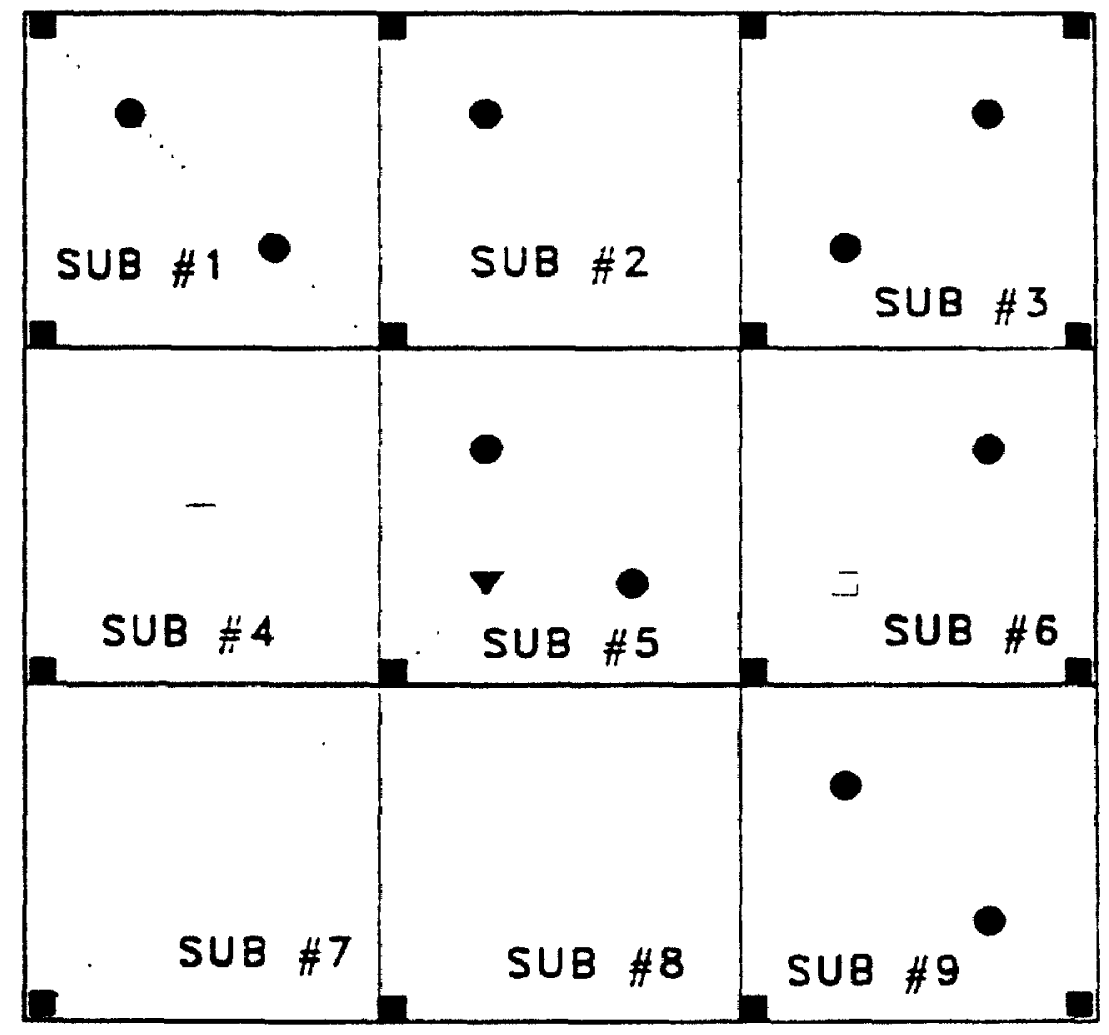

- UNCONFINED COMPRESSION TESTS

$\because$ CIU TRIAXIAL TESTS

$\checkmark$ ATTERBBERG LIMITS TESTS

Z CONSOLIDATION TESTS

- WATER CONTENT TESTS

NOTE : CHEMICAL TESTSAT VICINITY OF H! -V ELECTPODE

FIGLRE 6.8 EXPERIMENTAL DESIGN: POST-TREATMENT SOIL TESTHG 

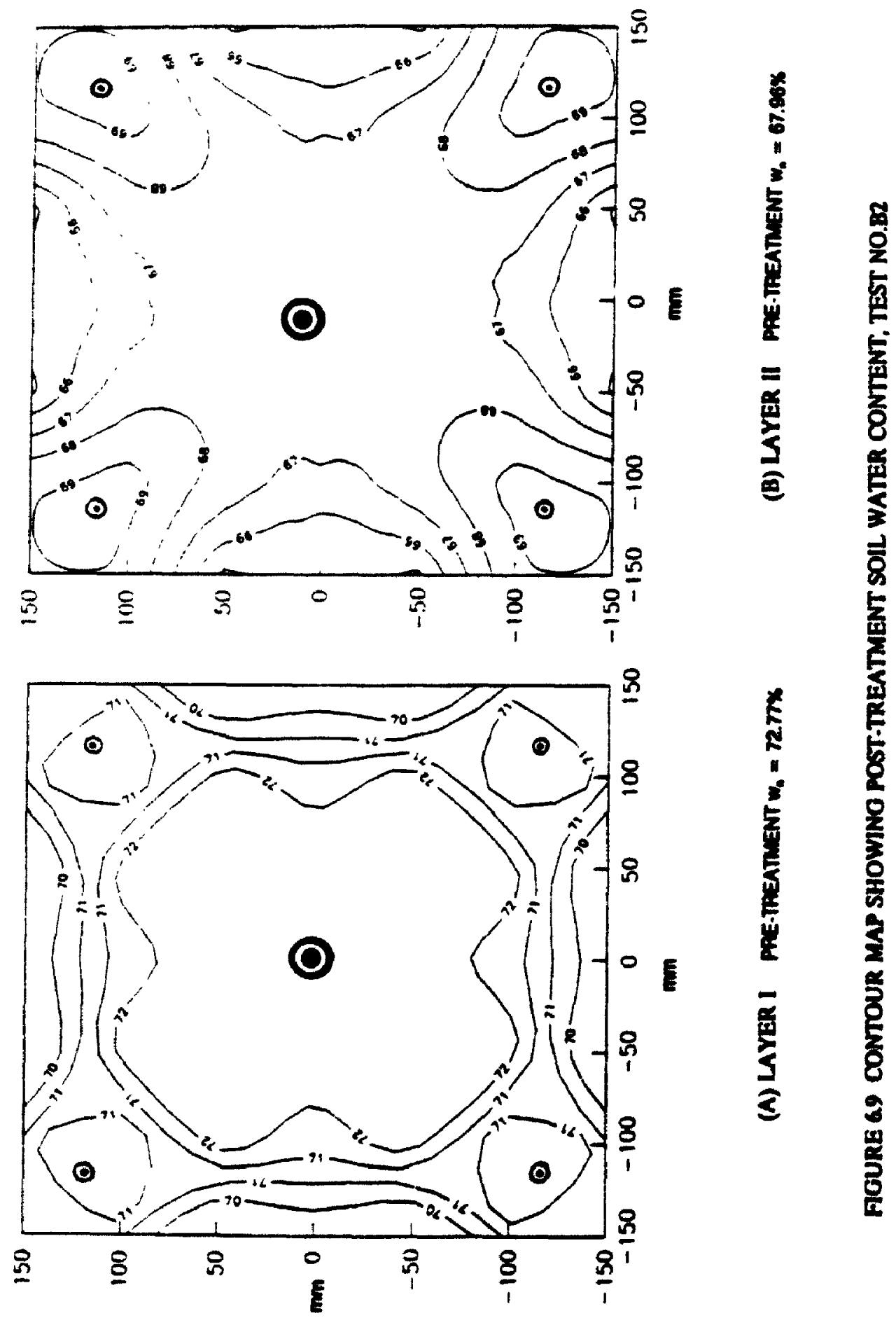


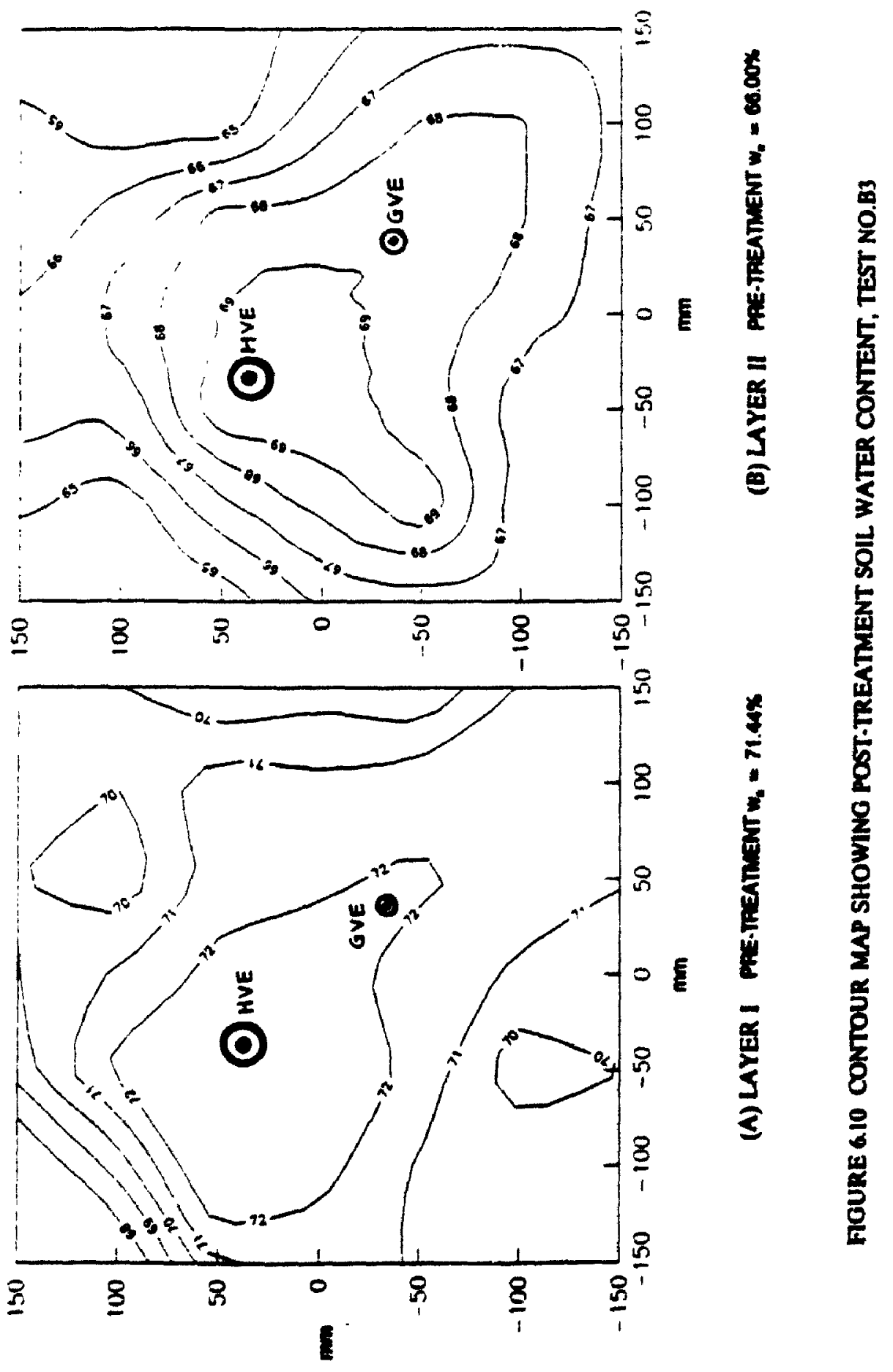


188

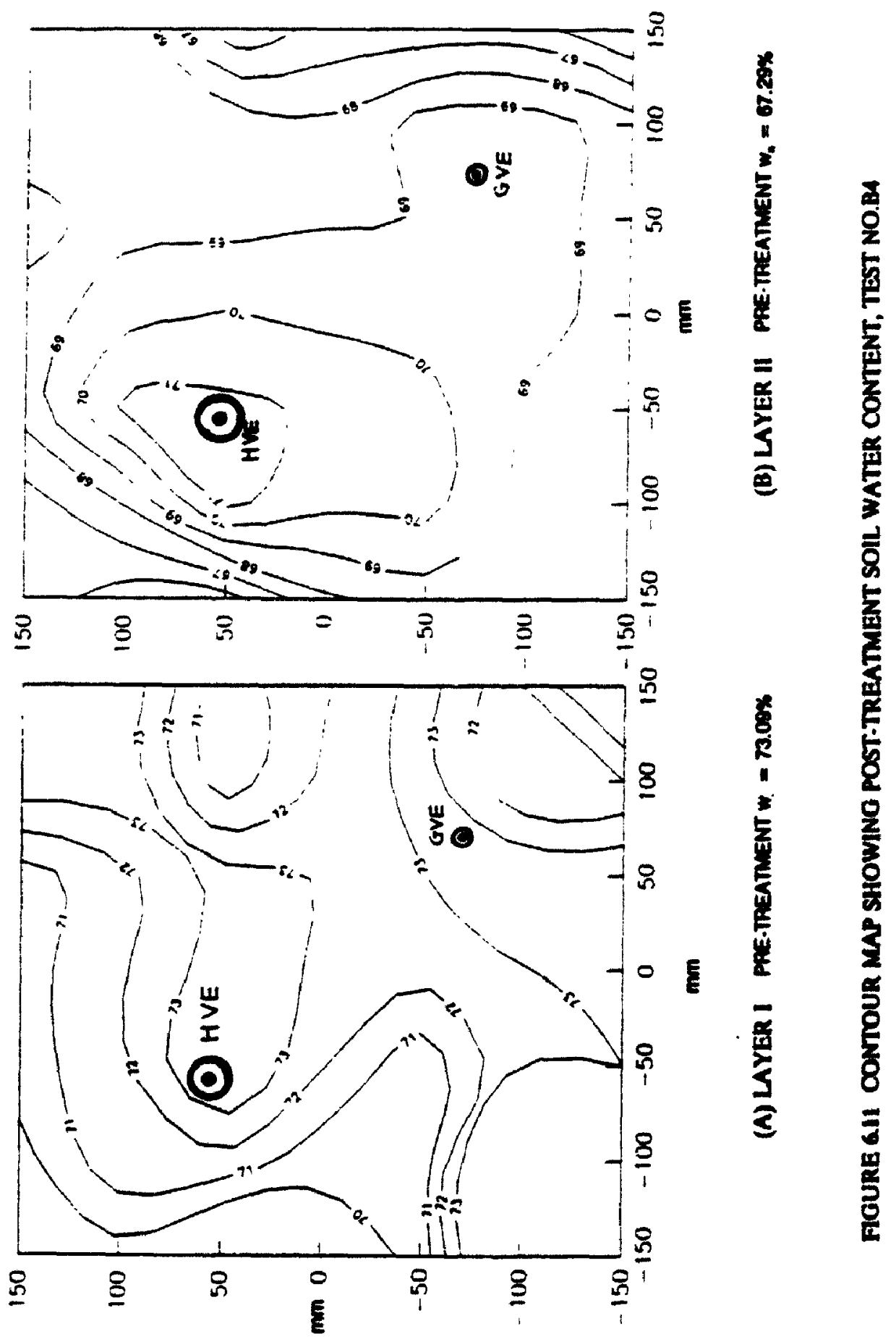




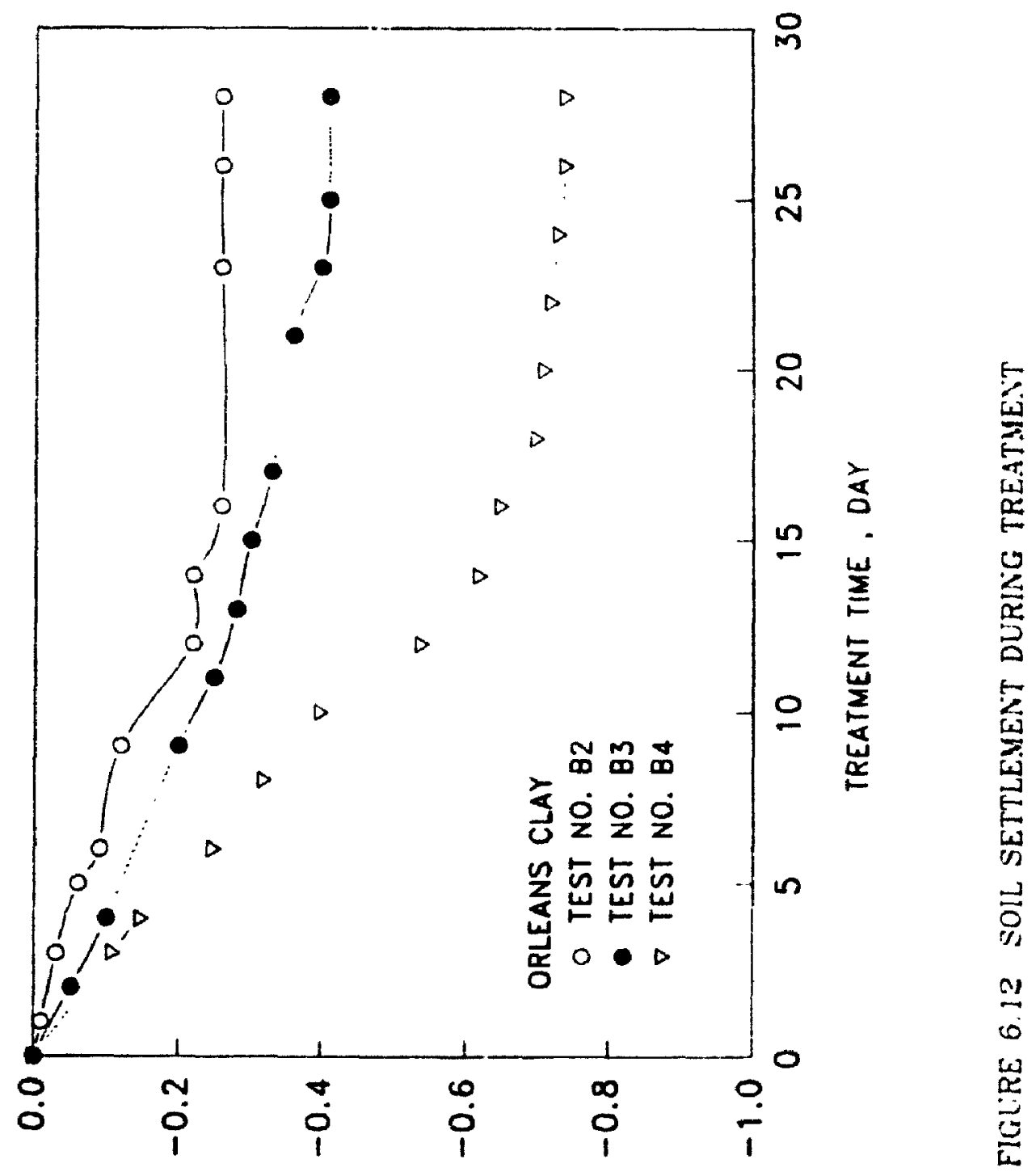

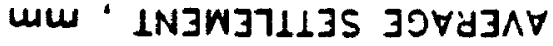




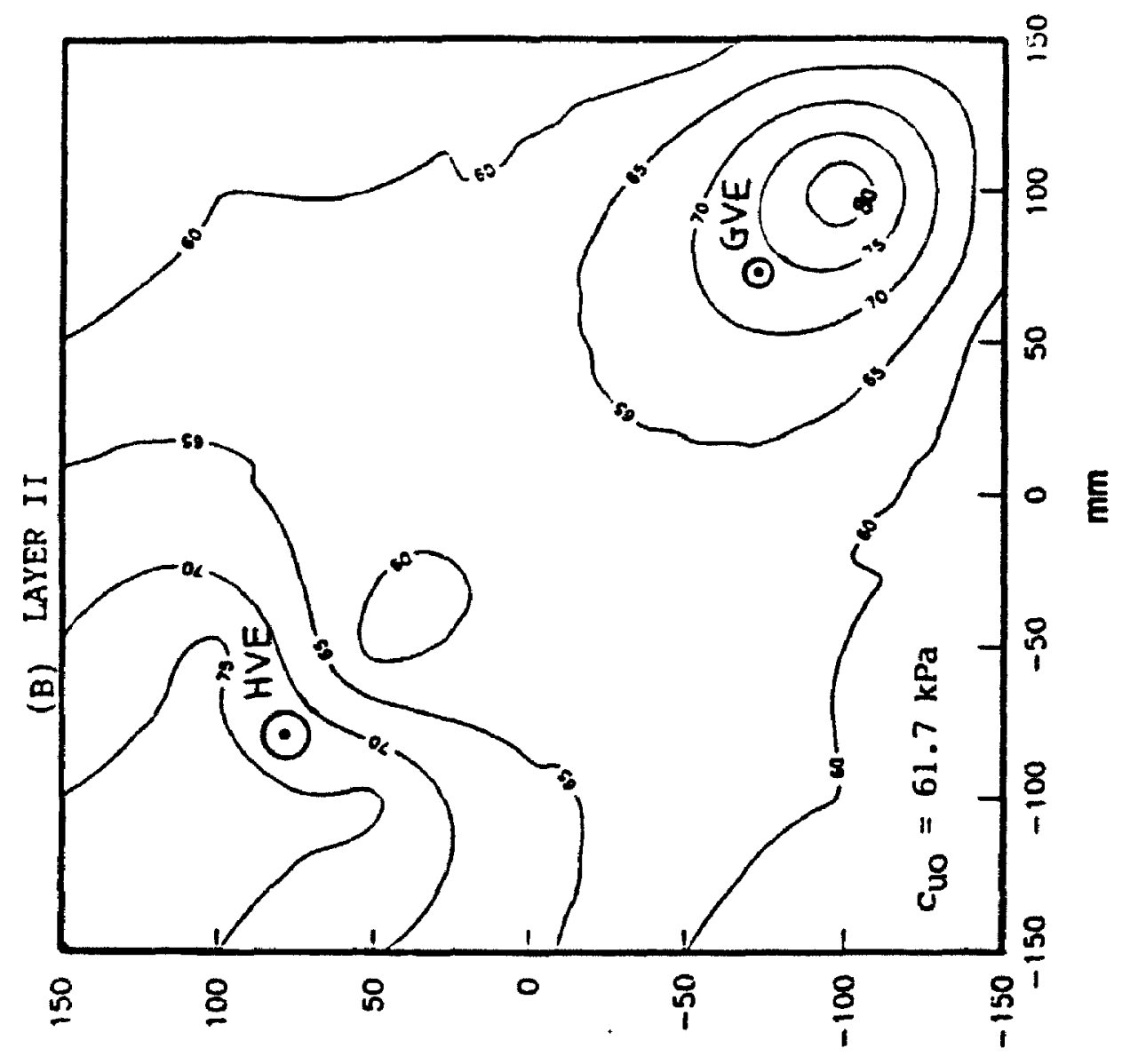

\section{0}

足

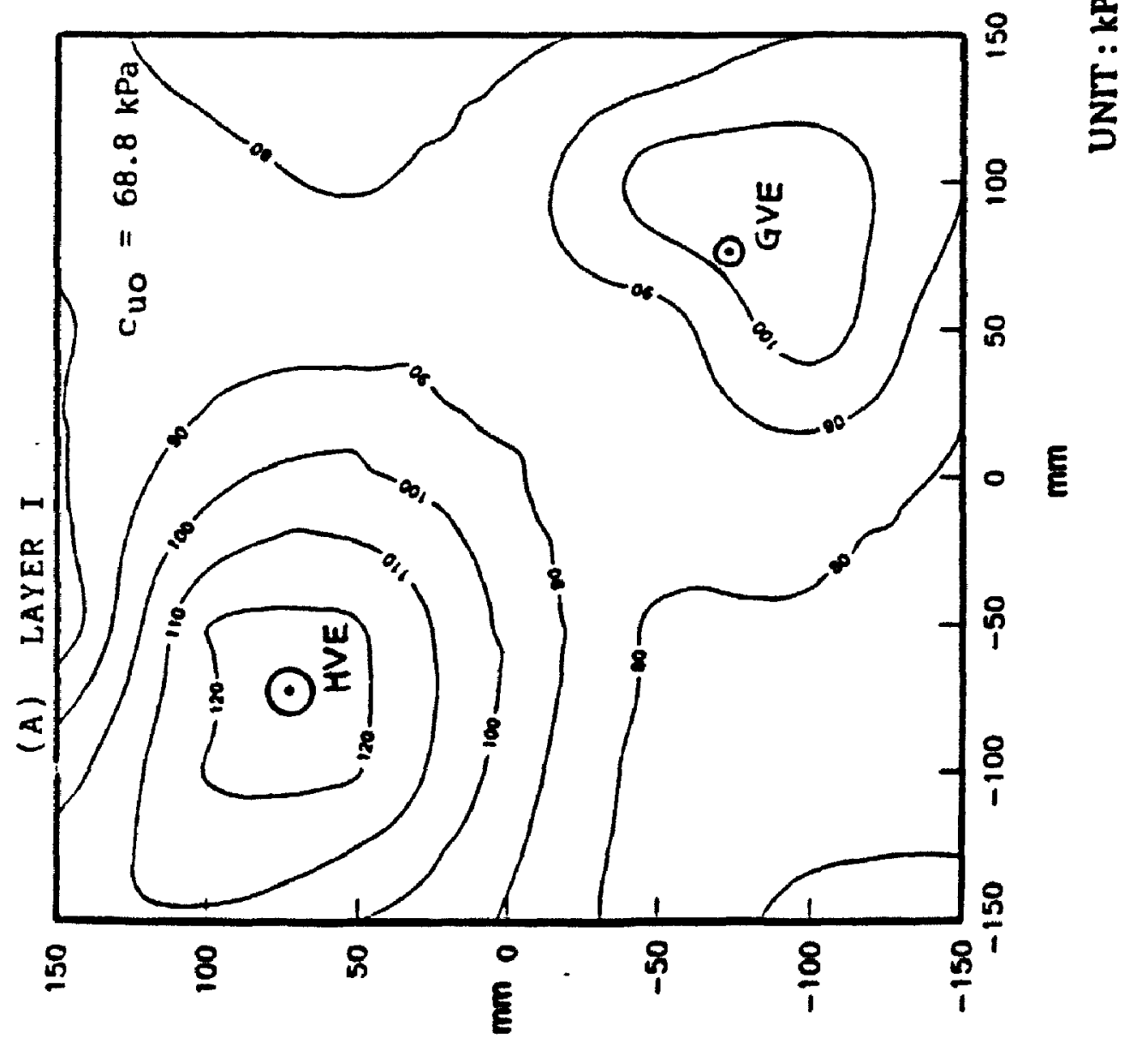




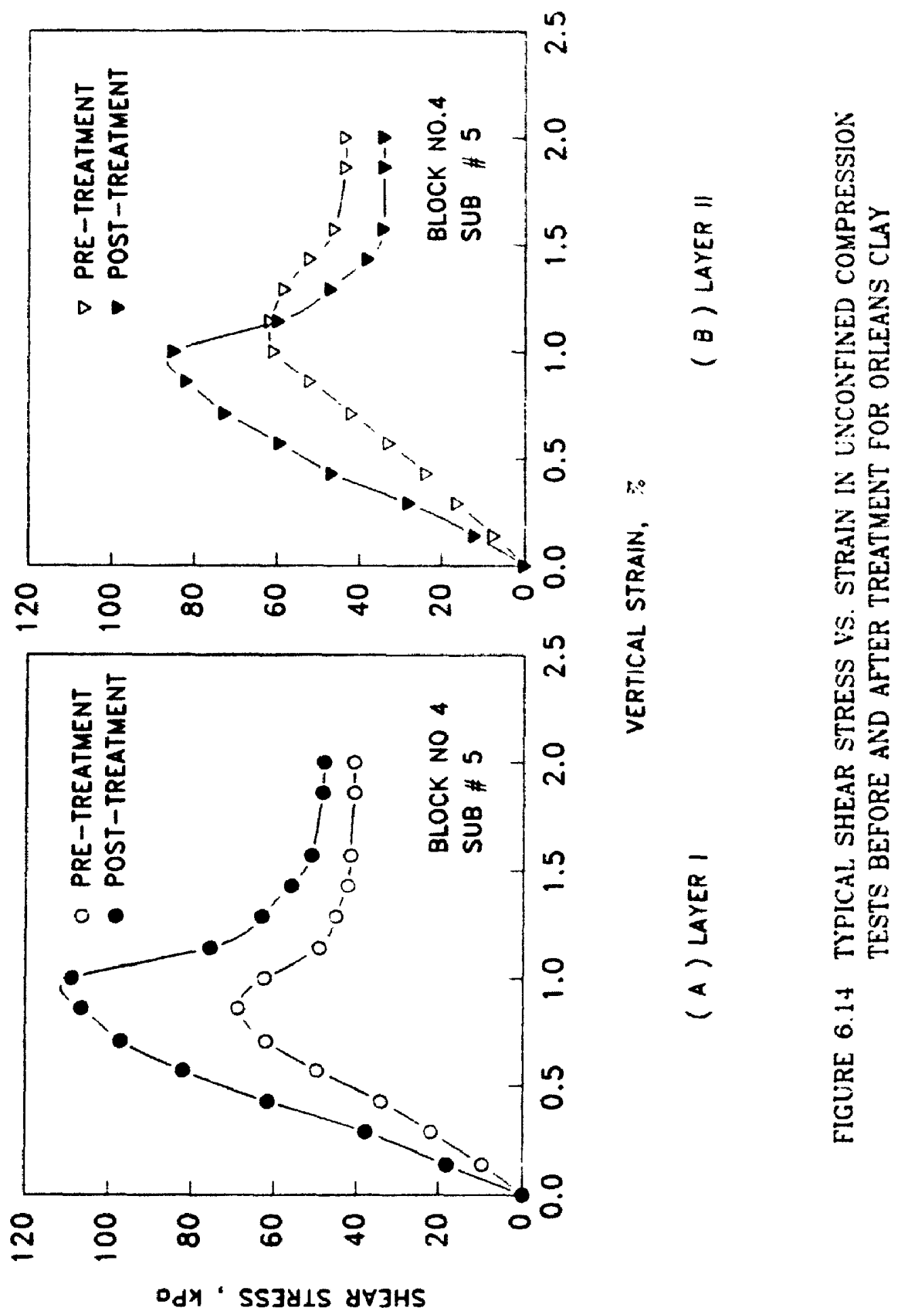



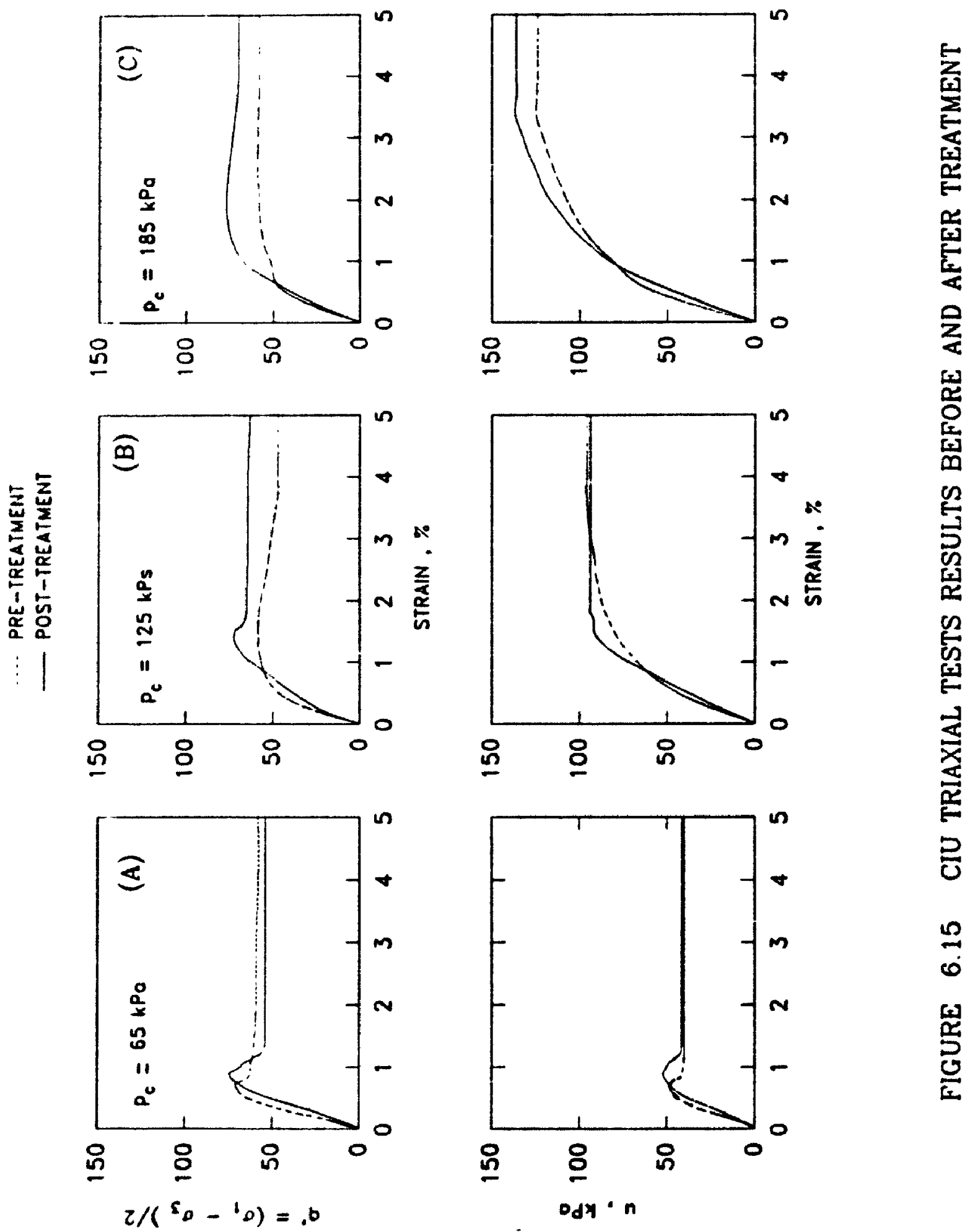


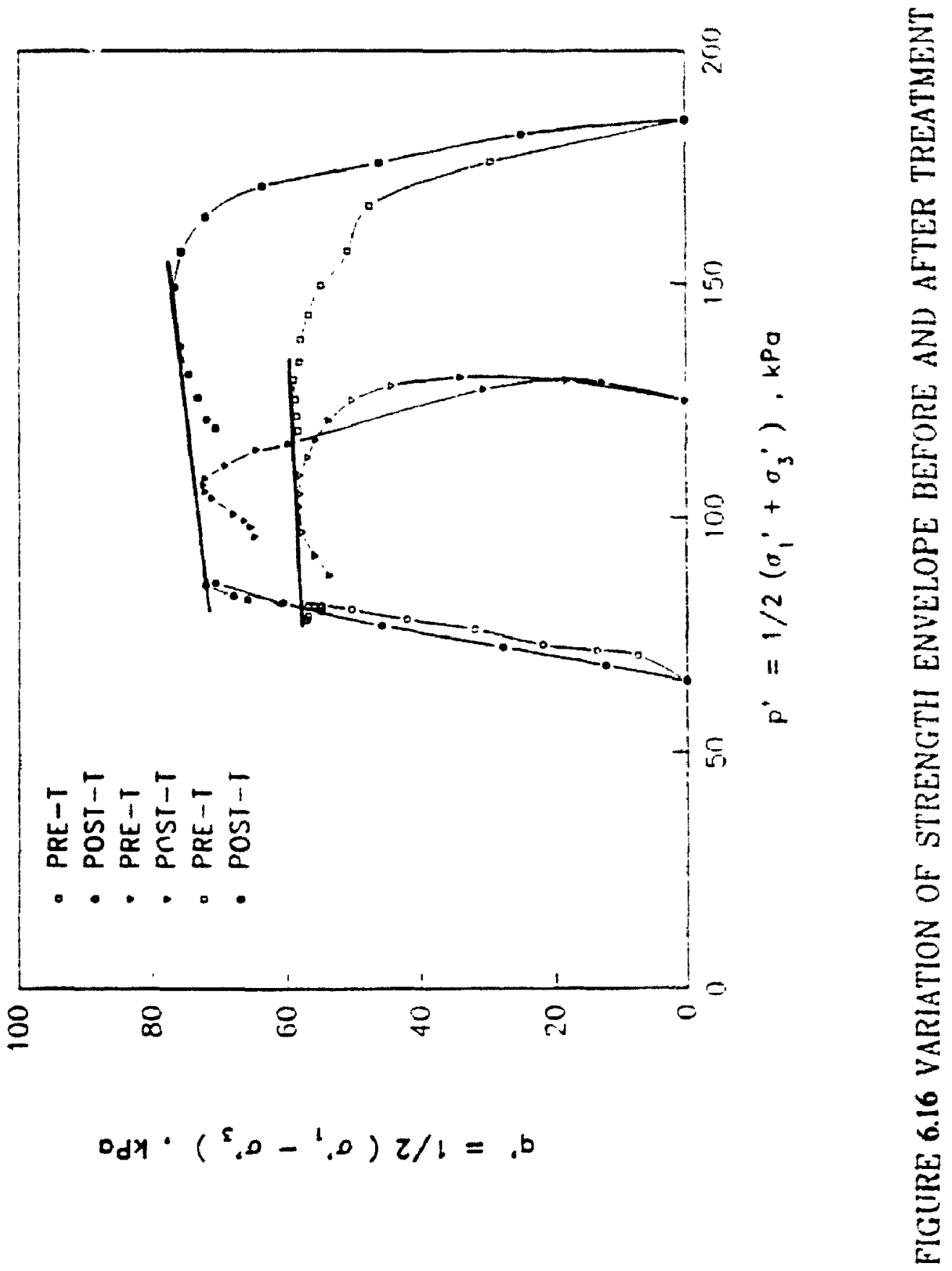




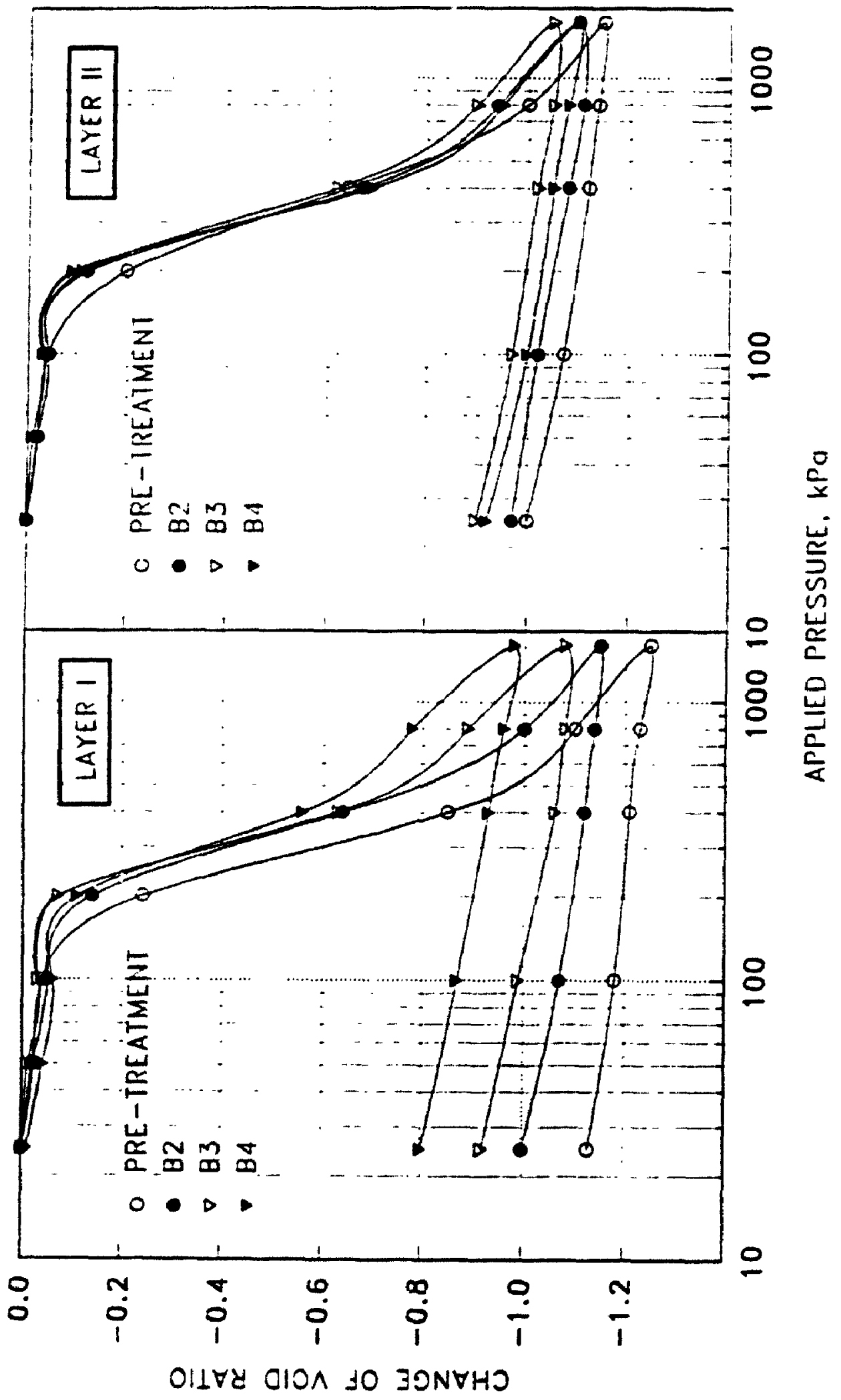

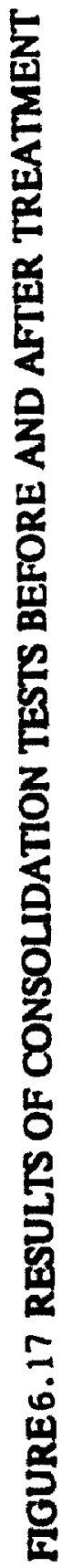




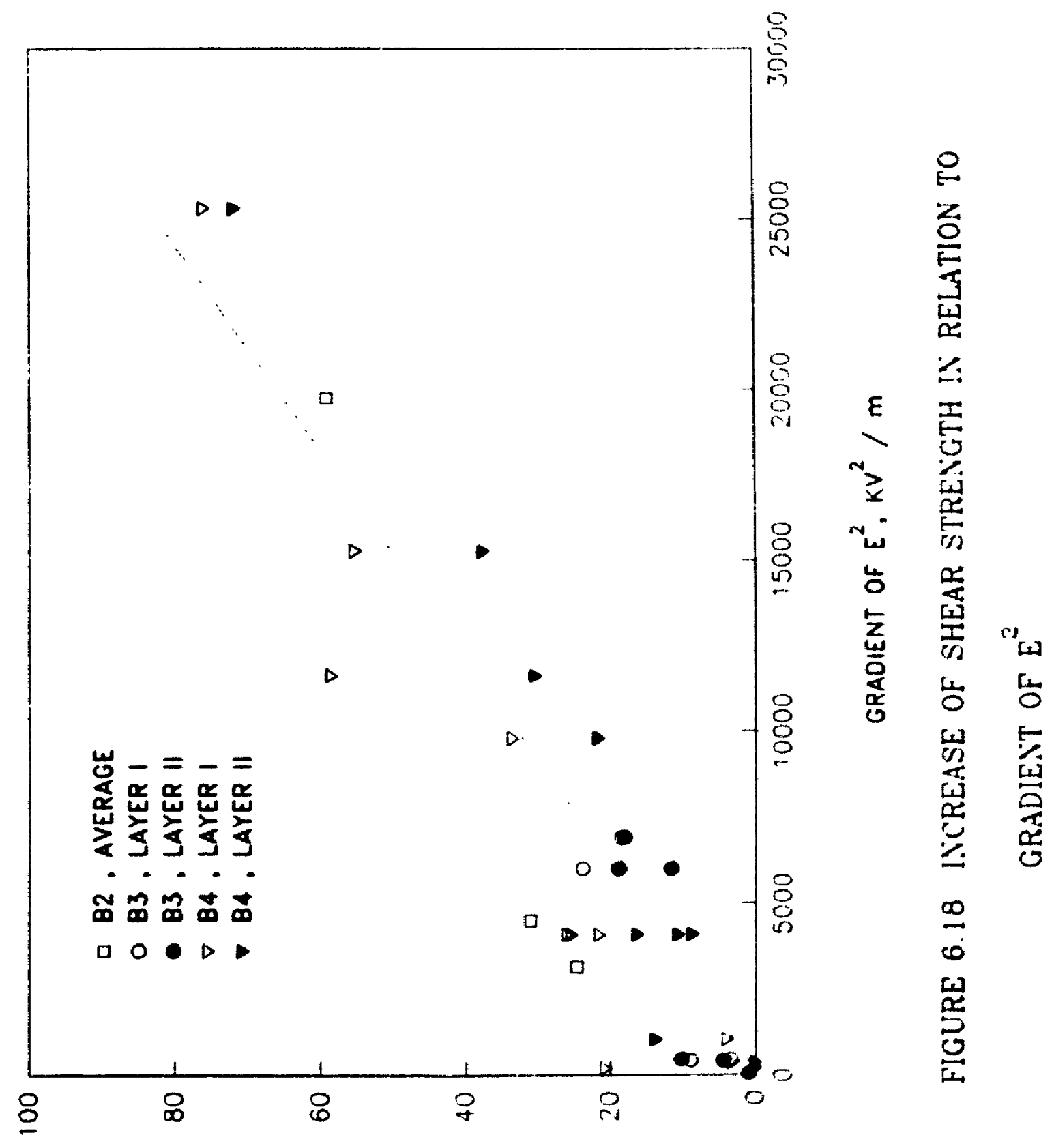

\% "HLONJYIS \& 


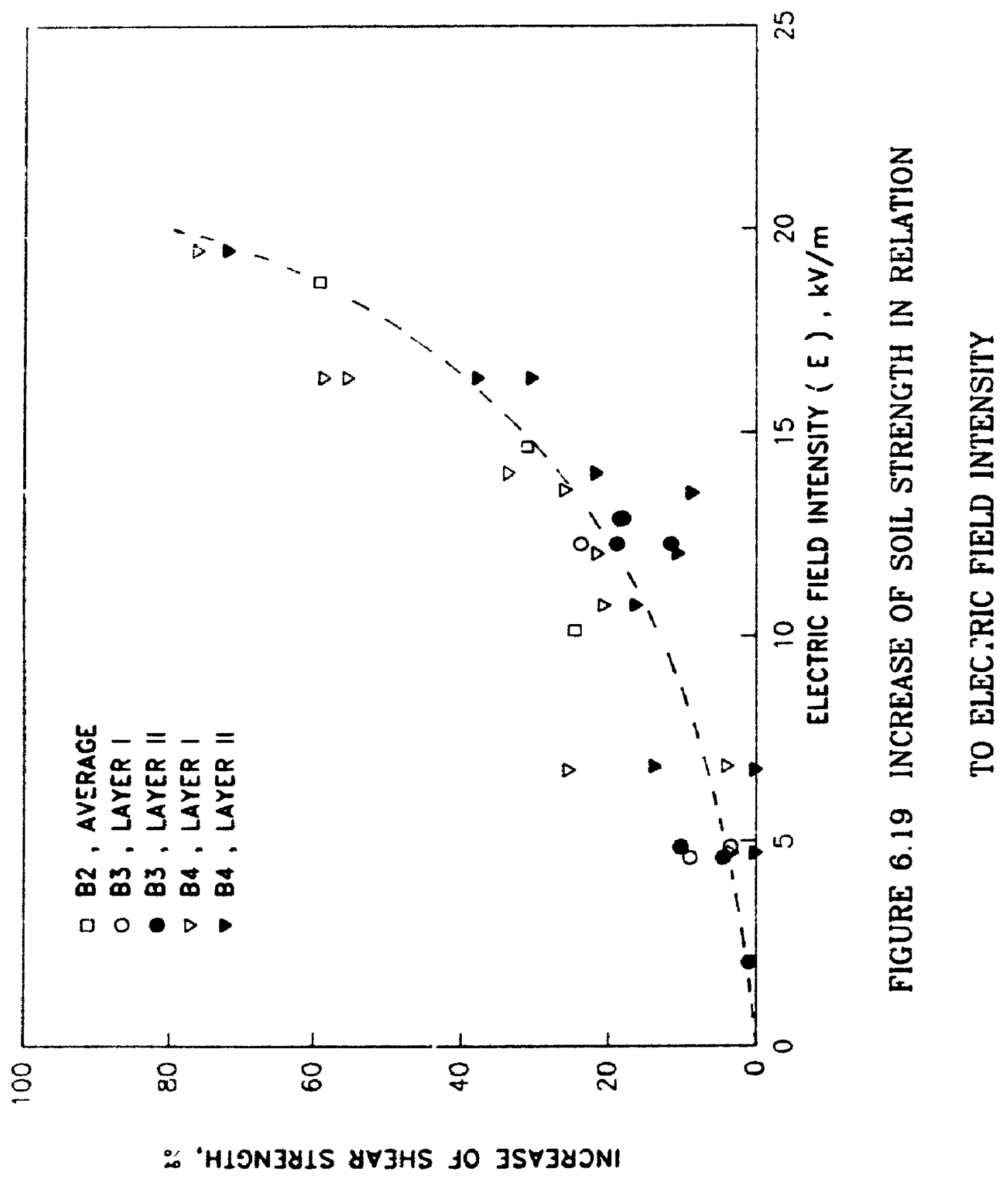




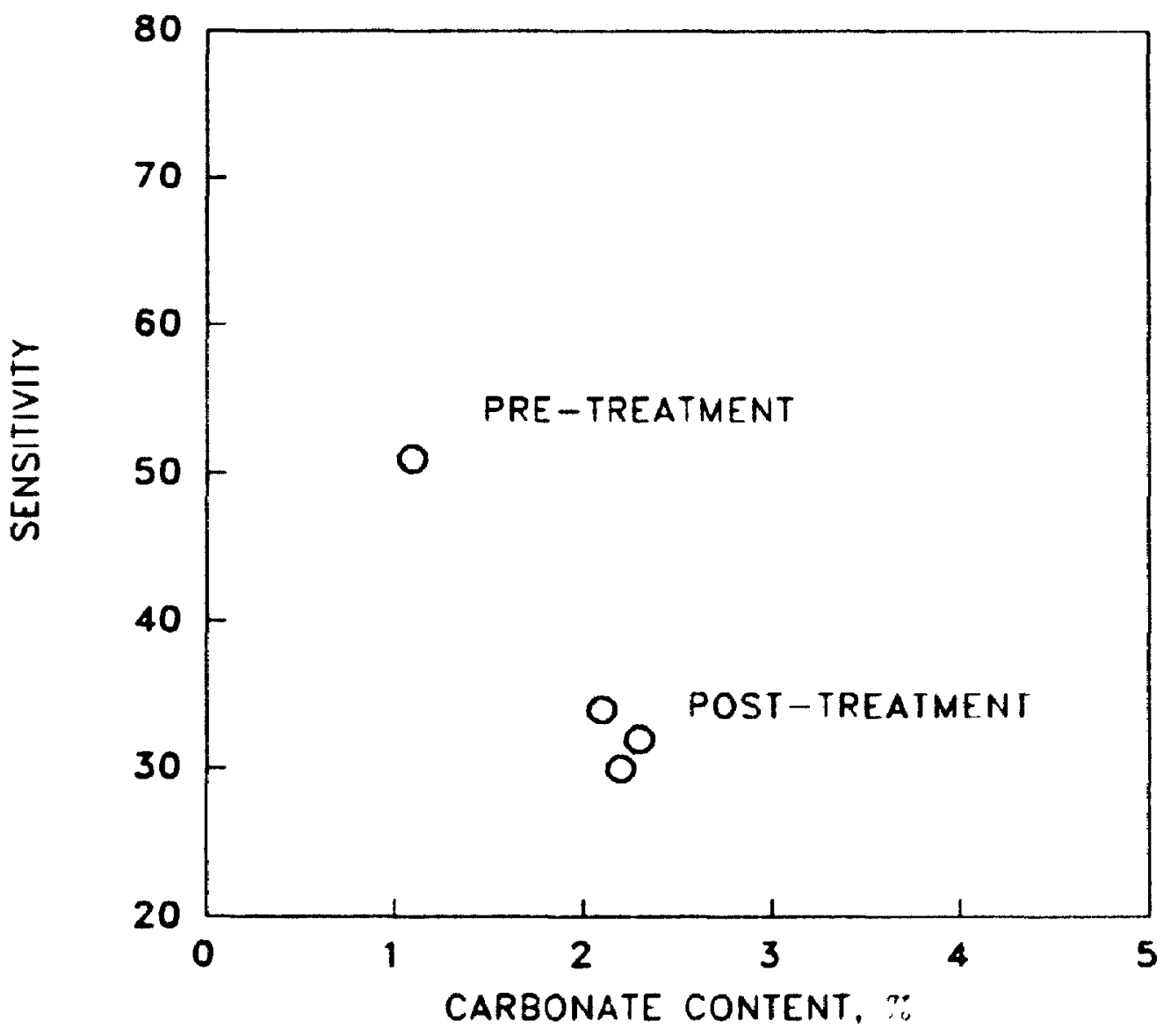

FIGURE 6. 20 SOIL SENSITIVITY VS. CARBONATI: CONTENT 


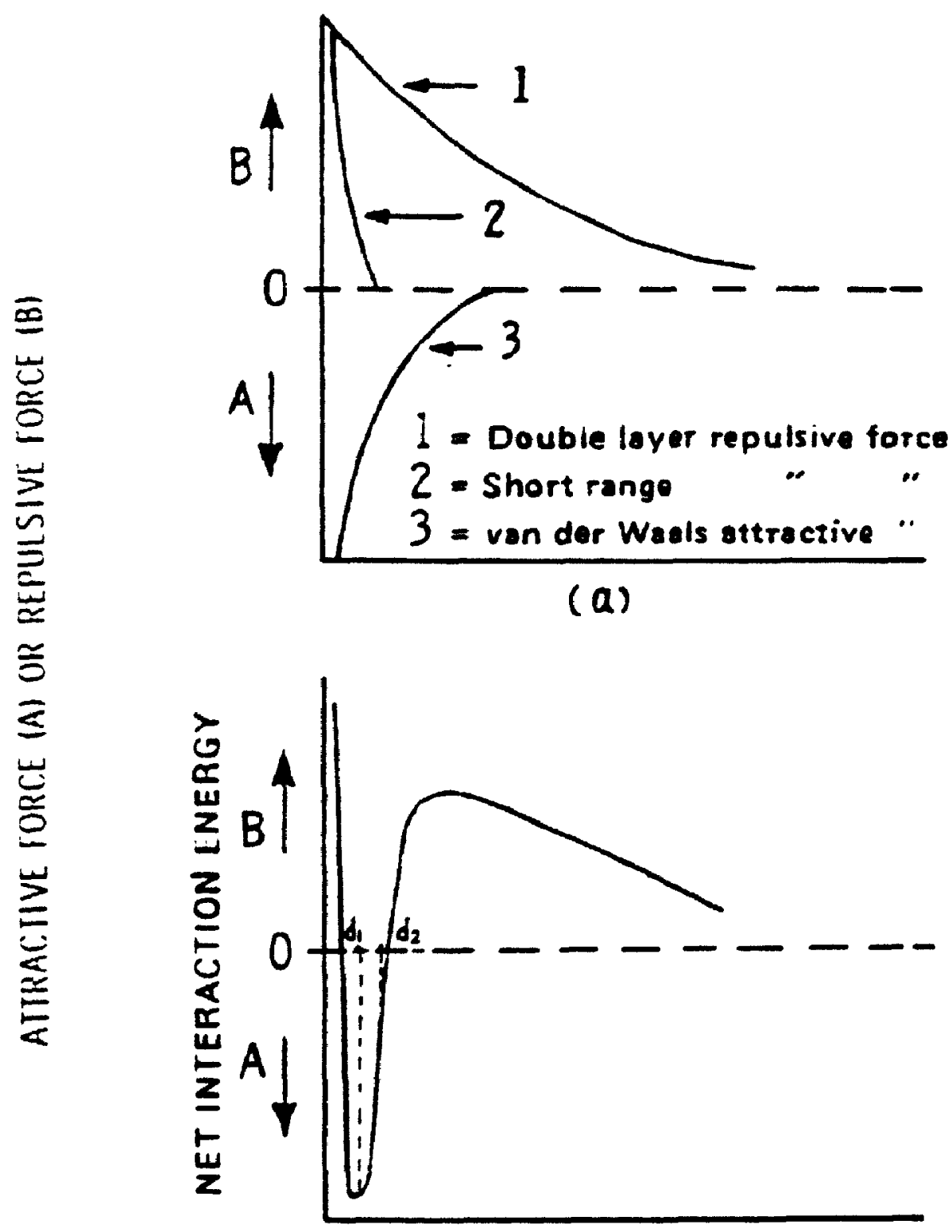

(b) 


\section{ChAPTER 7}

\section{DLECTRO-OSMOSIS IN ORLEANS CLAY:}

\section{A COMPARATIVE STUDY}

\subsection{Introduction}

The electrical strengthening of natural clays may be classified in two categories: electro-osmosis and dielectrophoresis. The principles and applications of electro-osmosis have been investigated, see e.g. Casagrande $(1941,1952)$, Mitchell (1976, 1991) and Lo et al (1991a, 1991b).

In order to further understand the mechanisms and compare the characteristics of dielectrophoresis and electroosmosis, a series of electroosmotic experiments on the Orleans clay was conducted. The results were discussed in conjunction with an electroosmotic experiment on the Gloucester clay conducted by Ho (1990) to study the effectiveness of electroosmosis in relation to the soil properties. Based on the experimental data, the characteristics of electroosmosis and dielectrophoresis were analyzed.

\subsection{An Overview on Flectroosmosis and Dielectrophoresis}

After a detailed study on dielectrophoresis, it is helpful to compare the processes of dielectrophoresis and electro-osmosis in clay soils.

Dielectrophoresis arises from the polarization of matter by means of the action of a non-uniform electric field. Dielectrophoresis can arise on both neutral particles and charged particles with electrical double layer. 
Electro-osmosis arises from the direct action of an electric field upon charged particles. It can happen regardless the uniformity of electric field. When an electric field is applied across a wet soil mass, cations are attracted to the cathode and anions to the anode. There is an excess of cations in the system to neutralize the net negative charge on the soil particles. As these cations migrate to the cathode, they drag water with them, causing water movement towards the cathode.

Some of the contrasts between dielectrophoresis and electro-osmosis are given below:

(1). Dielectrophoresis imposes a force on particles suspended in a fluid medium. In most clay-water-electrolyte systems, the dielectrophoretic force is imposed on clay particles towards the direction of lower field intensity.

Electro-osmosis is an action on cations in the pore fluid of a soil mass. The cations are attracted towards the cathode and drag water molecules along, inducing negative excess pore water pressure.

(2). Dielectrophoresis is a field-effect, i.e., its action is realized by a non-uniform electric field. Therefore, dielectrophoresis requires a relatively high electric field intensity generated by a high electric potential. The magnitude of the dieketrophoretic stress is determined by the nature of the clay-water-electrolyte system and the configuration of electric field. For applications with insulated electrodes, dielectrophoresis is primarily a physical process.

Electro-osmosis is basically a current-effect which requires reli.tively low electric field intensity. The electroosmotic consolidation is generated by the change of pore pressure. The direct electric current and the associated water flow are the 
key factors in the process. In the process, the metallic electrodes must be in contact with the soil in order to conduct the current. The process is associated with electrochemical reactions.

(3). Dielectrophoresis usually requires quite divergent electric fields for strong effects. Electro-osmosis operates in both uniform and divergent fields.

\subsection{Review of Theory}

The theoretical background of electro-osmosis has been well established. A mathematical model for electroosmotic flow was developed by Helmholtz in 1879 and was later refined by Smoluchowski (1914). The model indicates that there exists a double layer immediately adjacent to the capillary wall in a capillary tube, as shown in Fig-7.1 (Casagrande 1952). The thin inner layer consists of strongly adsorbed anions. The outer layer contains movable cations. Immediately adjacent to the double layer is free water. Owing to the application of electric current, the mobile positive ions will be attracted toward the cathode, dragging along with them the free water molecules, resulting in general movement of water from anode to cathode.

For one-dimensional electro-0smosis flow, Esrig (1968) showed that, by applying the continuity equation and the law of conservation of mass, the conventional one-dimensional consolidation equation will also hold true for onedimensional electroosmotic consolidation. For the system of closed anode and opened cathode, the pore water $u(x, t)$, in $\mathrm{kPa}$, at position $x$ along the sample at time $t$ is given by 


$$
\begin{aligned}
u(x, t)= & -\frac{k_{c}}{k_{k}} \gamma_{w} V(x)+ \\
& +\frac{2 k_{c} \gamma_{w} V}{k_{k} \pi^{2}} \sum_{n=0}^{-} \frac{(-1)}{\left(n+\frac{1}{2}\right)^{2}} \sin \frac{\left(n+\frac{1}{2}\right) \pi x}{L} \\
& \exp \left[-\left(n+\frac{1}{2}\right)^{2} \pi^{2} T_{v}\right]
\end{aligned}
$$

where $V(x)$ is the electric potential at position $x$ relative to the potential at cathode $(x=0), V$ is the maximum potential $(V), r_{w}$ is the unit weight of water $\left(\mathrm{kN} / \mathrm{m}^{3}\right)$, $L$ is the length of sample (m), $x$ is the distance from cathode $(m), T_{v}$ is the time factor in one-dimensional consolidation theory, $k_{\mathrm{h}}$ is the hydraulic permeability $(\mathrm{m} / \mathrm{s})$, and $k_{e}$ is the electroosmotic permeability $\left(m^{2} / s V\right)$.

As $t$ approaches infinity

$$
u(x, \infty)=-\frac{k_{c}}{k_{k}} \gamma_{\infty} V(x)
$$

Eq.(7.2) indicates that for one-dimensional electroosmotic consolidation, the induced negative pore-water pressure at any point in the soil during treatment is proportional to the potential at that point relative to the potential at $x=0$ (cathode).

Eq.(7.2) can be further developed by applying Ohm's law (Ho, 1990) 


$$
\begin{aligned}
V(x) & =-\int_{0}^{x} E(x) d x \\
& =i R(x)
\end{aligned}
$$

where $E(x)$ is the electric field intensity $(V / m)$, i is the current $(A)$, and $R(x)$ is the resistance of soil at position $x(\Omega)$. Substitute (7.3) into (7.2), we obtain

$$
u(x, \infty)=-\frac{k_{d}}{k_{h}} \gamma_{w} i R(x)
$$

It may be seen from Eq.(7.4) that the induced negative pore water pressure at equilibrium is a function of the electric current and resistance of the soil.

\subsection{Test Set-Up}

The electro-osmosis cell is the same one used in a previous study (Lo et al, 1991a). The cell is made of $101.6 \mathrm{~mm}$ plexiglas tubing of $304.8 \mathrm{~mm}$ length bounded by a top and a bottom plexiglas plate, as shown in Fig.7.2. The copper plate electrodes are placed at the top and bottom of the soil sample. A loading piston equipped with a dial gauge for measuring settlement is provided at the top of the cell and rest on the top electrode. Drainage can be controlled at both ends of the cell. The pore water probes (Fig.7.2) are used to measure the negative pore water pressure along the sample. The voltage distribution along the sample is measured using enamel wires as voltage probes. The interior of the cell is coated with a layer of silicon grease to seal the gap and to reduce friction between the cell wall and the soil sample. 
The test consists of two stages: the consolidation stage and the electroosmotic treatment stage. The sample was consolidated to the effective overburden pressure $(60 \mathrm{kPa})$ by increments. A back pressure of $40 \mathrm{kPa}$ was applied during the test. The electroosmotic stage was started after the consolidation of the sample was virtually completed (pore pressure dissipated 95\%). During the application of the electric potential, settlement of the sample, voltage distribution and pore water pressure at intermediate locations were monitored. The polarity of the electrode was reversed after the specific time period and the test proceeded at the reversed polarity for the same electric potential and the same time period. The whole process was then repeated at a higher electric potential. After treatment, laboratory tests were carried out to determine the change of soil properties.

Among the two Leda clays used in this study, the Gloucester clay has been thoroughly investigated (Ho, 1990, Lo, et al, 1991a). Therefore, the test was conducted on the Orleans clay. The properties of the Orleans clay have been described in Chapter 4. The Orleans clay is of the Leda clay's origin with the characteristics of sensitive clays, Table 4.1.

\subsection{Brocedure and Obaervation}

The soil samples used in the tests had identical dimensions, i.e., $101.6 \mathrm{~mm}$ diameter and $203.2 \mathrm{~mm}$ length. Two tests were performed. Each test lasted 4 days. During the test period, the anode was closed and cathode open to drainage. The applied voltage and polarity during test period are summarized in Table 7.1 (1). The predicted maximum pore pressures, i.e., the pore presoure at equilibrium $(t \rightarrow \infty)$ for 
the voltage applications are also listed in Table 7.2, estimated from Eq.(7.2). The electroosmotic permeability was estimated by Ho (1990) as $2.0 \times 10^{-6} \mathrm{~cm}^{2} / \mathrm{Vs}$ for the Gloucester clay and assumed to be applicable to the Orleans clay. The hydraulic permeability was determined as $3.0 \times 10^{-8} \mathrm{~cm} / \mathrm{s}$, Table 4.1. In Fig. 7.3, the positive current implies the normal polarity, i.e., the anode at the top and the cathode at the bottom of the electro-osmosis-cell and vice versa. In test EO-1, the voltage polarity was reversed every 24 hours, whereas in test EO-2 after 48 hours. The voltage increments were $4.0 \mathrm{~V}$ and $6.1 \mathrm{~V}$ in test EO-1. Test EO-2 was controlled according to the electric current. The applied voltage was higher on test EO-2 than that on test EO-1, as indicated in Table 7.1 (1).

It was observed that cracks developed on at the top and bottom of the soil sample in test EO-1. The cracks started at the region between the electrode and soil from day- 2 and expanded with time. At the end of day-4, the lengths of cracks were about $6 \mathrm{~cm}$ at the top and $3 \mathrm{~cm}$ at the bottom. When the sample was extruded from che cell after treatment, it broke into pieces at the top and bottom. Hence the consolidation and unconfined compression tests could be performed only on the middle portion. In test EO-2, a few minor cracks were observed between the top electrode and the soil sample at day- 2 , because of the disturbance by the pore pressure probe, as will be described later. The cracks, however, did not expand after the probe was withdrawn. No cracks were found at the bottom of the sample. The soil sample was generally intact after treatment.

The stainless steel pore pressure probes inserted into the soil sample interrupted soil settlement, causing cracks at the top of soil samples and had to be 
removed after the first 24-hour treatment.

\subsection{Rerults}

The test results are reported in terms of soil settlement, pore pressures at the first 24 hours, changes of water content, shear strength, stress-strain relation, undrained modulus, sensitivity, pre-consolidation pressure, physical and chemical properties at completion of tests. The results were compared and discuseed with an electroosmosis test (No. GV-8) on the Gloucester clay conducted by Ho (1990). The soil sample in test GV-8 had same dimensions with those in tests EO-1 and EO-

2. A summary of results for tests EO-1, EO-2 and GV-8 is presented in Table 7.1.

\subsubsection{Settlement}

The settlement-time relationships are presented shown in Fig7.4. In both tests, the settlements were very small, corresponding to the vertical strains of $0.125 \%$ and 0.435\% for the tests EO-1 and EO-2, respectively, Table 7.1 (2). The small settlement in the Orleans clay was consistent with the results of dielectrophoretic experiments, Fig.6.12, attributed to the stifiness of the clay.

The settlement in teat GV 8 was presented in Fig. 7.5. As seen the settlement was significant, representing a vertical strain of $13.52 \%$, Table 7.1(2).

\subsubsection{Negative Pore Water Prescure}

The development of negative pore-water presoure at the end of the first 24 hour was shown in Fig.7.6. It is observed that the pore presure is the highest at the 
cathode and approaches zero at the anode, consistent with the distribution of voltage along the sample length. The measured maximum pore pressures at the sathode were $20.5 \mathrm{kPa}$ in test EO-1 and $36.2 \mathrm{kPa}$ in test EO-2, respectively.

The theoretical distribution of pore pressure at equilibrium $(t \rightarrow \infty)$ was estimated from Eq.(7.2) and presented in Fig.6. It may be seen that at the end of 24-hour treatment, the pore pressures have reached the equilibrium in the three tests. We see from Fig.7.6 that a higher pore pressure was induced in test EO-2, attributable to the higher applied voltage and consequently, the higher electric current through the soil.

The pore pressure distribution in test GV-8 was presented in Fig.7.7. Again, we see that the agreement between theory and experiments is fairly good.

A difference between tests EO-1, GV-8 and test EO-2 is that in the former two tests, the voltage polarity was reversed once the equilibrium of pore water pressure was approached while in the latter test the treatment was carried on at the same polarity for another 24 houre. The significance of the difference will be discussed in next section.

\subsubsection{Soil Water Content}

The water content of each sample after treatment was measured at five locations along the length of the sample. Figure 7.8 presents the distribution of water content along the length. It is found that for both samples, the overall change of water content was very small, Table 7.1 (2). In sample EO-1, as shown in Fig.7.8 (A), the water content is noticeably higher at the middle portion and lower at the 
two end portions. This observation may provide a good indication to the process. The electro-osmosis treatment was divided into 4 equal time periods of 24 hours in tests EO-1 and GV-8. The voltage polarity was reversed at the end of each period. The pore water was driven towards the cathode during treatment. However, the rate of water flow was not compatible to the polarity reversal. In other words, the polarity was reversed before the water reaching the cathode. The overall effects were two folds: the first, the water accumulated in the middle portion of the soil sample causing the increase of water content, and the second, cracls developed at the two end portions due to desiccation and heating

The similar distribution was observed by Ho, as shown in Fig.7.9. The overall water content was reduced significantly due to the softness of the soil. However, the distribution of water contents shows the similar patten with that in test EO-1.

The water content distribution in sample EO-2 is shown in Fig 7.8 (B). The water content was reduced in the middle portion and increased in the two endh. The observation may further confirm the discusaion above. In test EO-2, the voltage polarity was reversed after 48 hours treatment, about 24 hours after the pore prescure equilibrium was reached. The water flow in the soil then could continue under the maximum negative pore pressure for a steady period of time. The water distribution after treatment suggests that the water had been transported to the two ends of the sample. The heating effect was partially compensated by the water driven to the electrodes 20 that no cracks were found. Overall a dewatering effect was fulfilled in the middle portion of the sample.

Here we may draw an important conclusion regarding the electro-osmosis 
process, i.e., the electroosmotic pressure should be maintained for a certain period of time after the equilibrium of pore pressure is reached to allow completion of water transport.

The changes of water content in the Orleans clay during both the electroosmosis and dielectrophoresis are small (Table 6.1). Again, this may be attributed to the stiffness of the Orleans clay.

\subsubsection{Shear Strength}

The shear strengths of the soil samples after treatment were determined by unconfined compression tests on $35 \mathrm{~mm}$ diameter and $70 \mathrm{~mm}$ length specimens. The shear rate for all tests was $0.5 \%$ strain per minute. As described before, sample EO1 cracked at two ends so that only one specimen could be trimmed from the middle portion. Sample EO-2 was intact in general after treatment, therefore three specimens were trimmed from the top, middle and bottom portion, respectively. Table 7.1(3) summarizes the results. The distributions of shear strength along the sample length are presented in Fig.7.8, in which we see that the change of shear strength is associated with the change of water content. Afu test EO-1, the shear strength decreased at the middle portion after treatment (from $61.9 \mathrm{kPa}$ to $47.2 \mathrm{kPa}$, a decrease of 23.7\%), as shown in Fig.7.8 (A). The detrease of shear strength may be interpreted by the increase of water content at this portion (from $65.0 \%$ to $71.4 \%$, an increase of $\mathbf{9 . 8 \% )}$.

The relationship between the shear strength and water content is shown further after test EO-2, Fig7.8 (B). The shear strength increased significantly in the 
middle portion of the sample (from $59.6 \mathrm{kPa}$ to $88.0 \mathrm{kPa}$, an increase of $47.7 \%$ ), associated with a decrease of water content ( from $69.7 \%$ to $67.4 \%$, a decrease of $3.3 \%$ ), whereas at the top and bottom of the sample, the shear strengths were less affected, associated with the small changes of water contents.

The change of shear strength associated with the change of water content was also observed in test GV-8. As seen in Fig.7.10, the shear strength after test GV-8 was lower at the middle and higher at the two ends of the sample, consistent with the water content distribution in Fig.7.9.

The strengthening effects of electro-osmosis and dielectrophoresis may be compared with the data summarized in Table 6.2 and Table 7.1 (3). The results in test B2 (DEP test) and test EO-1 (EO test) are omitted because of cracks. We may see from Table 6.2 that the average increase of shear strengths after dielectrophoretic treatment were $44.0 \%$ for test B2 and $24.6 \%$ for test B4, respectively, at small changes of soil water content. The average increase of shear strength was $17.3 \%$ after test EO-2. It should be noted that it was actually no change of shear strength at the two ends of the sample, as shown in Fig.7.8.

The characteristic of electroosmosis may be seen clearly through the results. In electro-osmosis process, the water flow is the key effect. The soil shear strength increases wherever the soil water content reduces and vice versa.

\subsubsection{Stress-Strain Curve, Undrained Modulus and Sensitivity}

The stress-strain curves from unconfined compression tests are presented in Fig.7.11. The corresponding peak stresses (shear strengths), strains at failure, 
brittleness indexes and undrained moduli are summarized in Table 7.1 (3). As shown in Fig.7.11 (A), after test EO-1, the specimen trimmed from the middle portion of the sample shows significantly decrease of undrained modulus. This could be attributed to the increase of water content resulting from the uncompleted pore water transportation. After test EO-2, Fig.7.11 (B), the peak of the stress-strain curves did not changed significantly, whereas the post-peak strength was increased noticeably for the specimens trimmed from the top and bottom portions of the sample. A significant strength increase is observed at the middle portion, associated with the decrease of water content.

The reduction of sensitivity is the most distinguishable change. The sensitivity of the soil reduced from 51 before treatment to the order of 20 after treatment, representing a decrease ranged from 55\% - 57\%, Table 7.1 (2). The similar result was obtained in test GV-8, as shown in the same table. The reduction of sensitivity by means of electrical techniques is the common characteristic of electro-osmosis and dielectrophoresis, as reported by Lo et al (1991a, 1992) and in this thesis. The decrease may attribute to the increase of carbonate content, as will be discussed later.

\subsubsection{Pre-Consolidation Pressure}

The consolidation tests were performed after treatment. The specimens were trimmed from the middle portion of the soil samples. The results are plotted in Fig.7.12 and the pre-consolidation pressures before and after treatment are summarized in Table 7.1(2). In general the shape of consolidation curves did not change significantly after treatment for both samples, as observed from Fig. 7.12 
The pre-consolidation pressurc after test EO-1 was slightly reduced, from $142 \mathrm{HPa}$ to $137 \mathrm{kPa}$. After test EO-2, the pre-consolidation pressure increased from $142 \mathrm{kPa}$ to $183 \mathrm{kPa}$, representing an increase of $28.9 \%$. Again we note that the increase of preconsolidation pressure is associated with the decrease of water content.

The action of negative pore pressure can be illustrated in Fig.7.12. The Orleans clay is an overconsolidated clay with the in-situ effective overburden pressure of $60 \mathrm{kPa}$. In test EO-1, the application of an average voltage of $5 \mathrm{~V}$ generated a negative pore pressure in the order of $30 \mathrm{kPa}$, as estimated from Eq.(7.2). The resulting overall effective stress was $90 \mathrm{kPa}$, which was below the preconsolidation pressure of the soil (142 $\mathrm{kPa})$. Consequently, the soil deformation was small and no significant changes in terms of strength and preconsolidation pressure. In test EO-2, the application of an average voltage of $8.3 \mathrm{~V}$ generated a negative pore pressure in the order of $50 \mathrm{kPa}$, resulting in an overall effective stress of $110 \mathrm{kPa}$, which again did not exceed the preconsolidation pressure.

The consolidation curves before and after test GV-8 were shown in Fig.7.13. The in-situ effective overburden pressure is $33 \mathrm{kPa}$ for the Gloucester clay. The application of an average voltage of $4.5 \mathrm{~V}$ in test GV-8 generated a negative pore pressure in the order of $40 \mathrm{kPa}$, generating an effective stress of $73 \mathrm{kPa}$, which was higher than the preconsolidation presoure of the clay (49 kPa). As a result, the soil was consolidated at a stress level higher than its preconsolidation pressure, causing large deformation, significant change of water content and strengthening of the soil. 


\subsubsection{Physical and Chemical Changes}

The chemical changes associated with electro-osmosis has been explored (Mitchell, 1976). During electroosmotic process, hydrogen gas is evolved at the cathode. Cations in solution are driven to the cathode where they combine with the (OH) - that is left behind to form hydroxides. Therefore the $\mathrm{pH}$ will rise at the cathode. At the anode, oxygen gas is evolved by hydrolysis, and anions in solution react with free $\mathrm{H}^{+}$to form acids, causing the $\mathrm{pH}$ decease at the anode. The high acidity and oxidation cause rapid deterioration of the anode,

Table 7.2 summarizes the results of physical and chemical tests before and after electroosmotic treatment. The increases of liquid limit and plastic limit after treatment were observed in both Gloucester clay and Orleans clay. The increase of liquid limit was relatively higher, resulting in the increase of plastic index. The carbonate contents increased significantly after treatment in both clays, which can be explained by electrochemical reactions, as discussed in Chapter 6 . The increase of carbonate content may provide a better bonding between clay particles and decrease soil sensitivity. The salinity did not change noticeably in the Orleans clay, due to the small water content changes. In the Gloucester clay, the salinity was increased after treatment, attributable to the decrease of water content.

The soil pH was measured from the top, middle and bottom of the Orieans clay samples after treatment. Decomposition of the bottom copper electrode was observed during treatment and the soil in contact with the electrode turned into a greenish colour. The soil pH values at the bottom were pH 6.7 for EO-1 and pH 6.6 for EO-2, indicating acid reactions. On the other side, the soil in contact with the top 
copper electrode was observed to turn into a brownish colour with pH 9.8 for EO-1 and $\mathrm{pH} 10.3$ for EO-2, respectively, showing that the alkaline reactions were dominant during treatment. The soil $\mathrm{pH}$ values in the middle portion of the soil samples had a moderate increase from $\mathrm{pH} 8.1$ to $\mathrm{pH} \mathrm{8.8.} \mathrm{The} \mathrm{results} \mathrm{indicate} \mathrm{that}$ in spite of the reversal of cathode and anode during treatment, a pH gradient was still generated. The pH value after test GV-8 was measured at cathode and was consistent with the values at the top electrode in Orleans clay test series.

The dielectrophoretic treatment has the advantage of eliminating the $\mathrm{pH}$ gradient and avoiding decomposition of electrodes. The changes of chemical properties are small due to insulation to the electrodes, as discussed in Chapter 6.

\subsection{Further Comparison of Electro-osmosis and Dielectrophoresis}

A quantitative cimparison between the experimental data on electro-osmosis and dielectrophoresis is rather difficult because the differences of the mechanism and parameters. However, we may compare the two processes by considering the following factors:

(1). Physical effect.

Electro-osmosis causing water flow is one of the characteristics of the process. Electro-0smosis draws water to a cathode, where it is removed from the system when no water is permitted to enter at the anode. The process relies on the hydraulic and electroosmotic permeability of clays. For the case of less compressible Orleans clay, the settlement was small but the water content re-distribution was still significant, indicating a water transport effect. 
The dielectrophoretic force, on the other hand, is applied directly on soil particles. The force is controlled by the electric field configuration. If the soil is relatively less compressible, as in the case of Orleans clay, the change of water content would be small during treatment.

(2). Electro-chemical effects.

Electro-osmosis requires direct contact between soil and conductor electrodes. Thus electrochemical reactions play important roles in the process. The effects include ion diffusion, ion exchange, $\mathrm{pH}$ change, desiccation due to heat generation at electrodes, mineral decomposition, electrolysis, hydrolysis, oxidation, reduction, physical and chemical adsorption and fabric changes. Some of these effects may be beneficial in terms of an electrochemical strengthening of soils whereas others, such as the deterioration of electrodes (anode) due to oxidation and pH gradient between cathode and anode and $\mathrm{pH}$ gradient, may raise environmental concerns.

Dielectrophoresis involves primarily physical effects. The electrochemical reactions are minimized attributed to insulation of electrodes. The possible physicochemical effects may arise as a results of water dissociation and the reaction of interparticle bonds due to the dielectrophoretic stress, as discussed in Chapter 6. In general, the potential problems in the environmental aspects are not prominent.

(3). Electro-osmosis is more time-efficient. The action of electro-osmosis is immediately associated with the electric current.

Dielectrophoresis may need longer treatment time because the process involves sustained action to achieve strengthening effect. 


\subsection{Concluxions}

The following conclusions may be drawn from the experimental investigation reported in this Chapter:

(1). The action of electro-osmosis is associated with the process of water flow in soil mass.

(2). The electroosmotic treatment should be carried on for a certain time period after the pore pressure equilibrium is reached to allow completion of water transport. Otherwise softening (strength reduce) may result, at least for stiffer clays.

(3). A pH gradient will be generated during electroosmotic treatment, which can not be eliminated through the reversal of voltage polarity.

(4). The changes of soil properties, including shear strength, pre-consolidation pressure and undrained modulus after electro-osmosis treatment are dominated by the change of soil water content, which is in turn controlled by the magnitude of negative electroosmotic pore pressure in relation to in-situ overburden pressure and preconsolidation pressure. To improve the soil properties significantly, the pore pressure generated should exceed the preconsolidation pressure.

(5). The major physical changes after electro-osmosis treatment include the increase of plastic index and decrease of sensitivity.

(6). The outstanding difference between electro-osmosis and dielectrophoresis is that in the former, the electric current is functioning as a carrier to transport soil water, whereas in the latter, the electric field applies a dielectrophoretic force on the clay particles directly and the strengthening effect is achieved by the sustained application of the non-uniform electric field. 


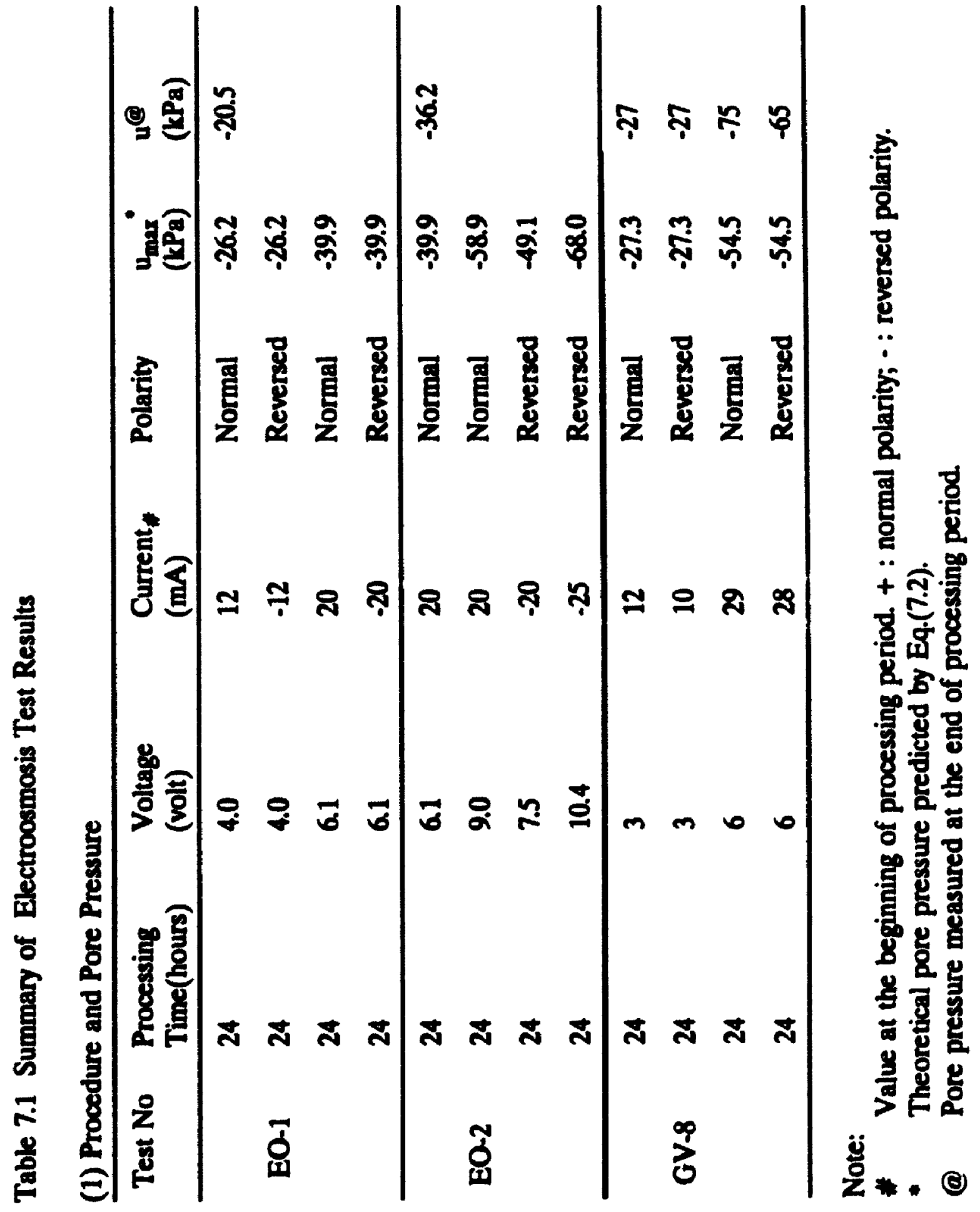




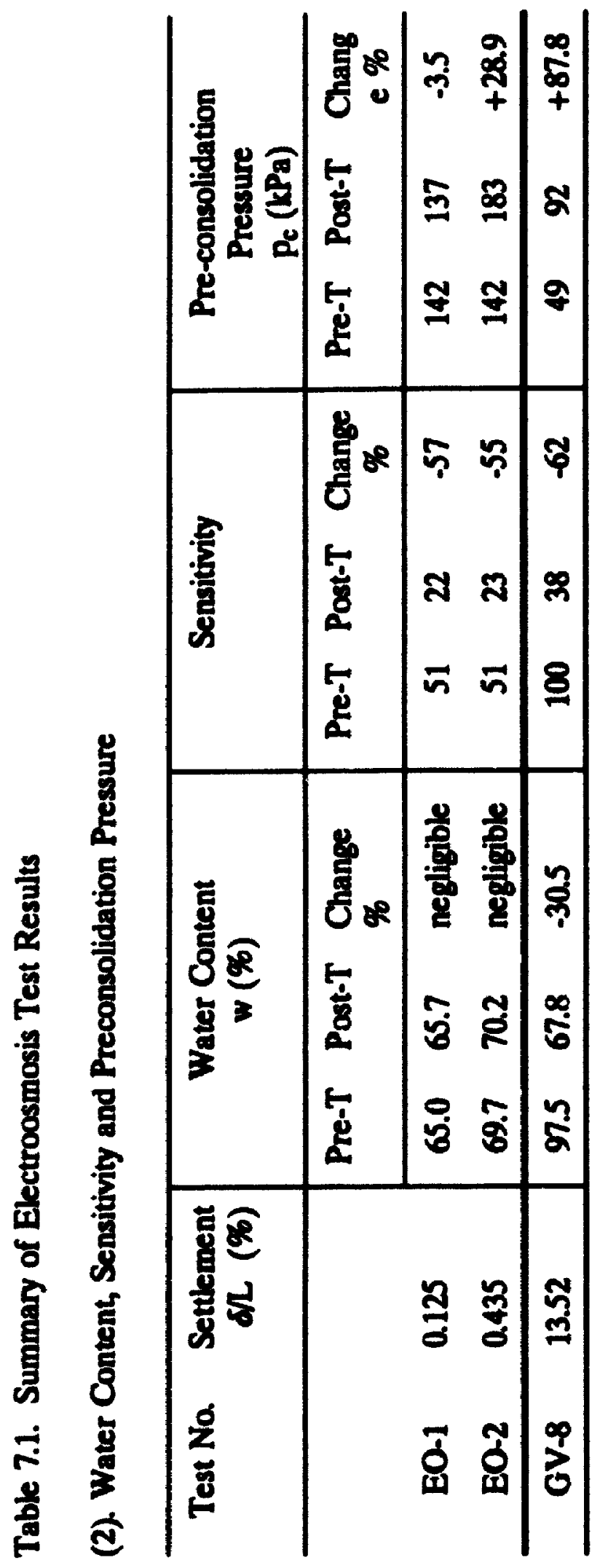




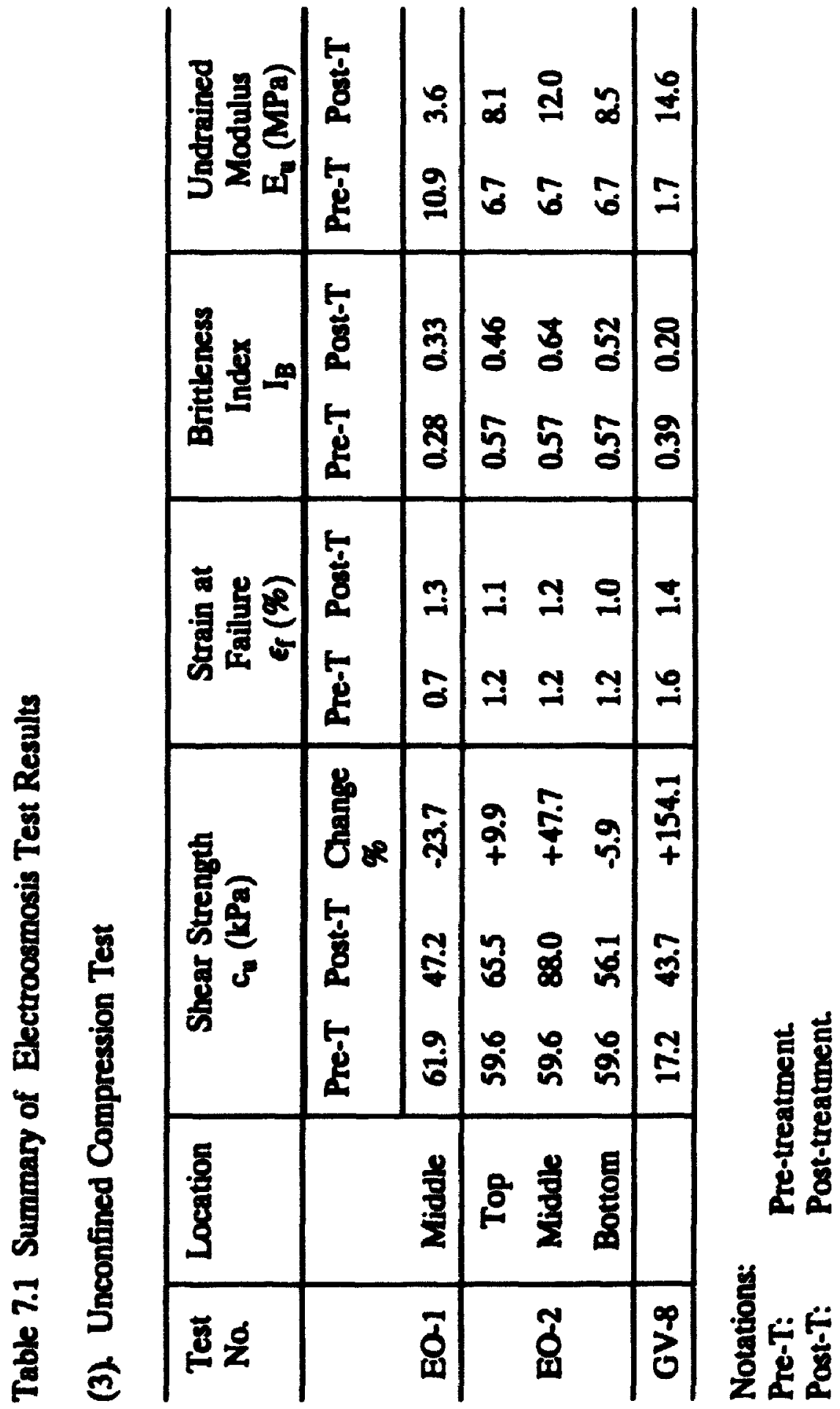




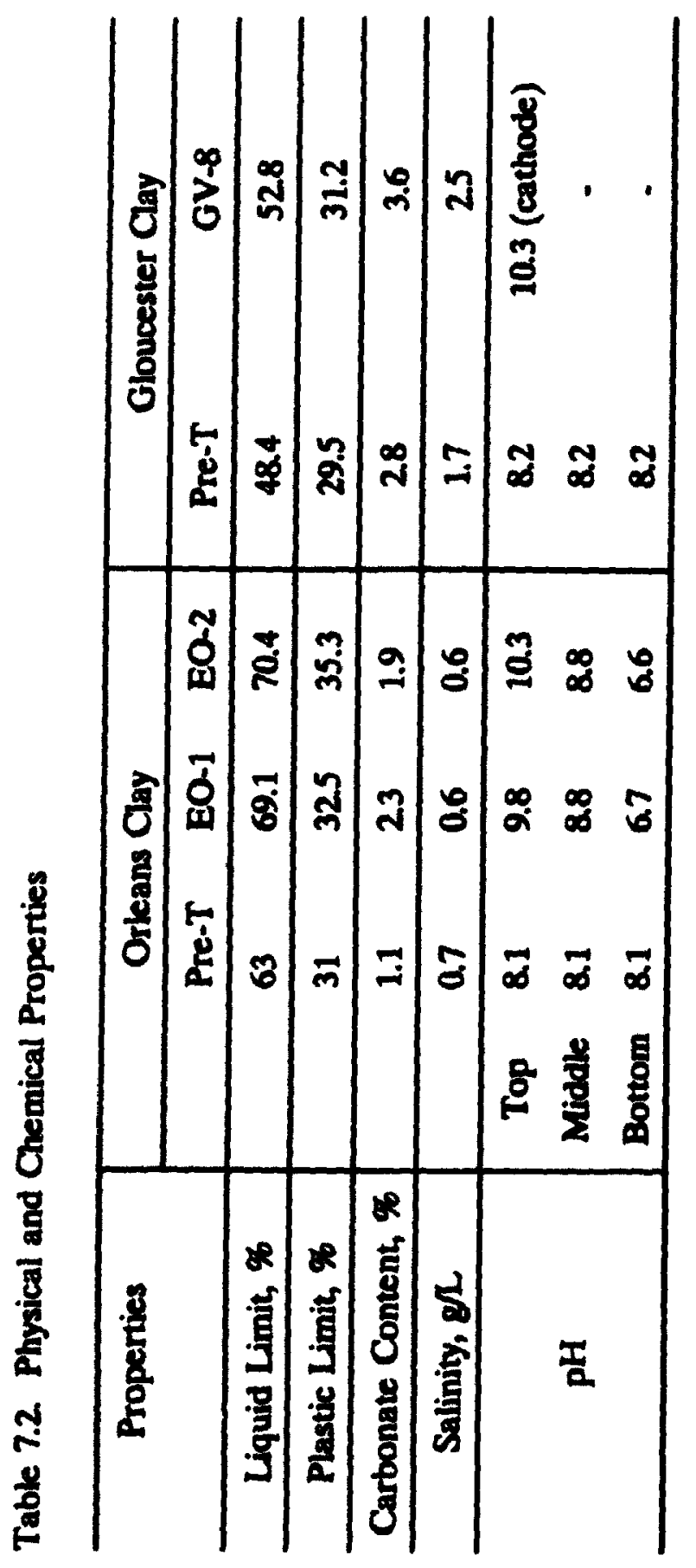




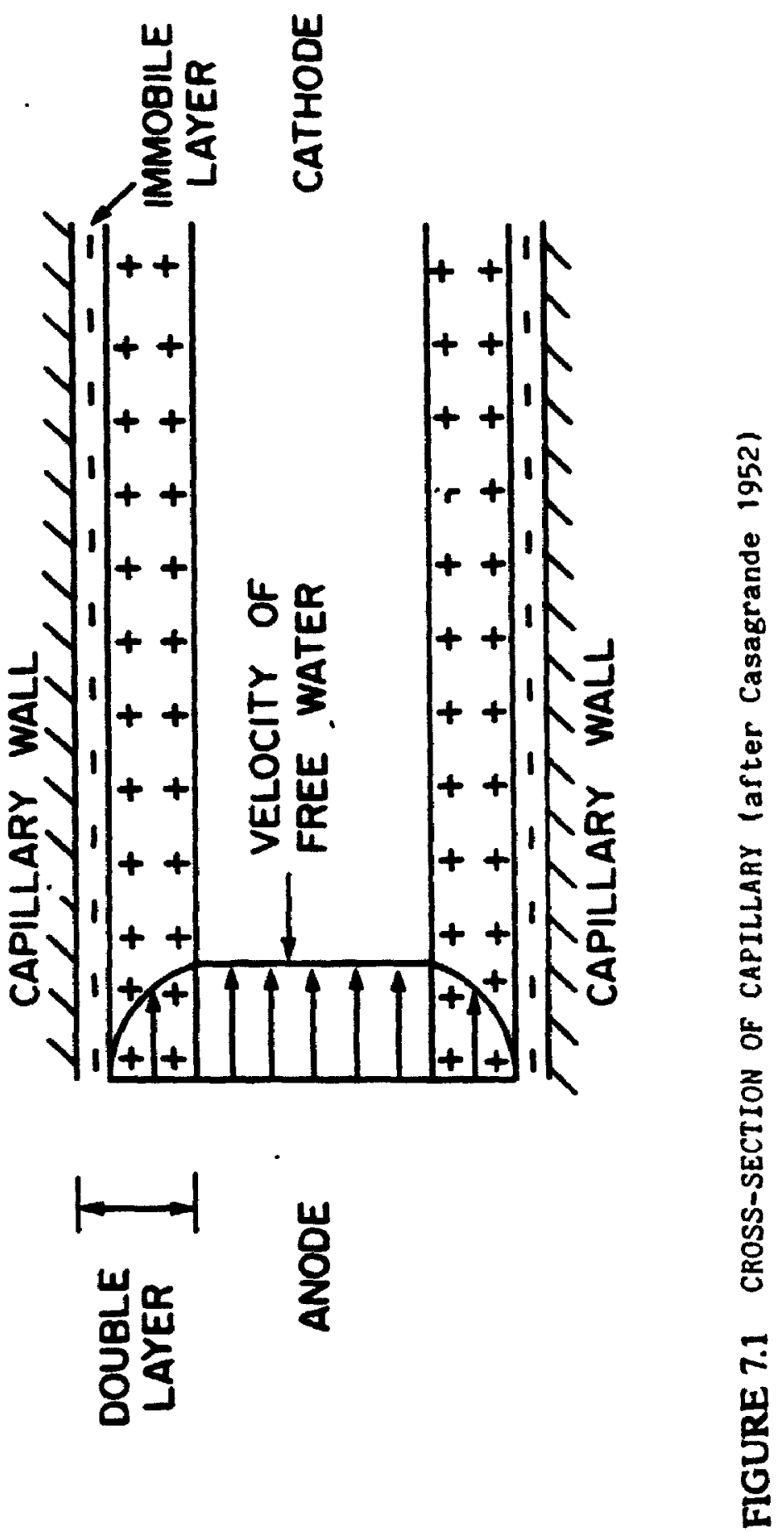




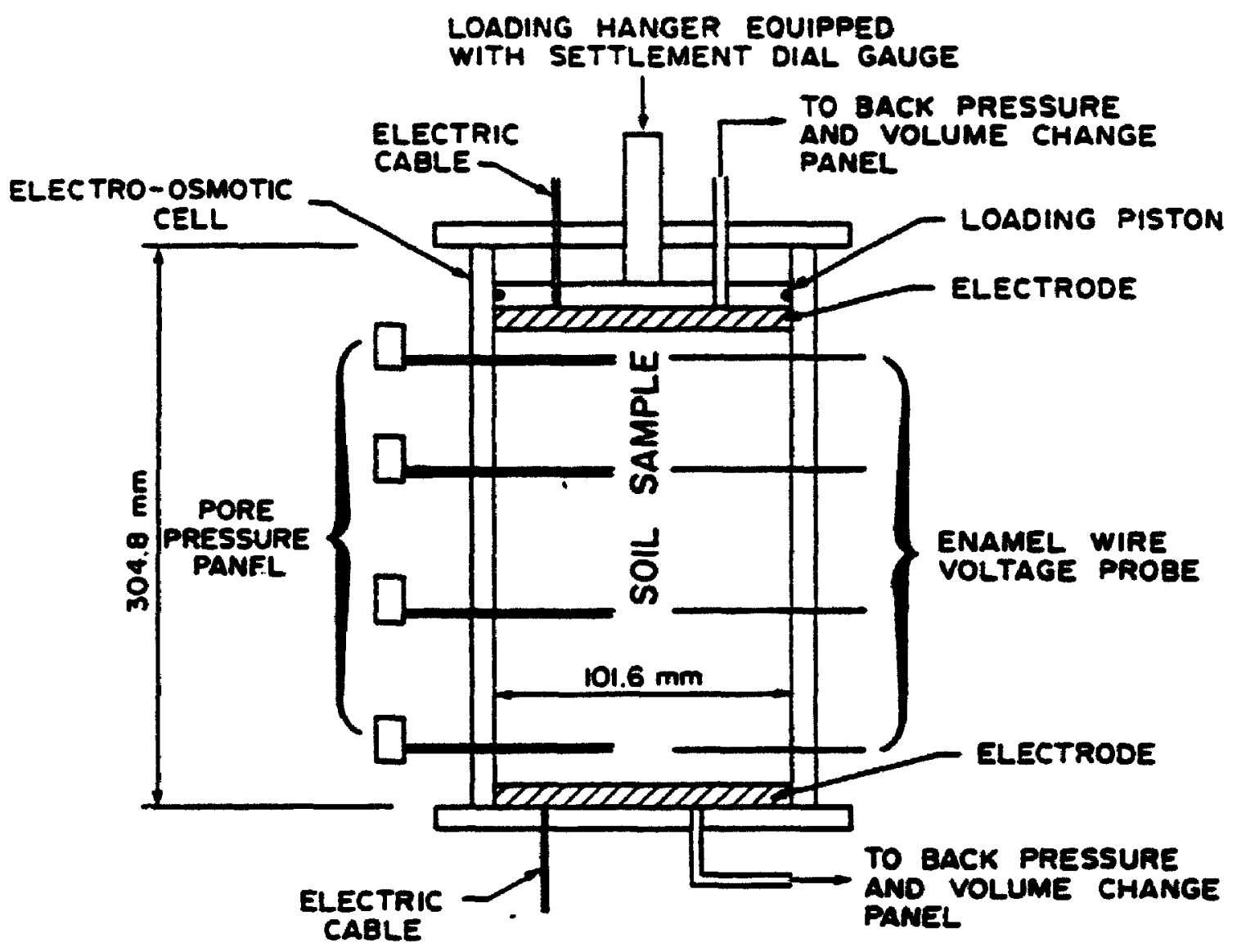

FIGURE 7.2 ELECTRO-OSMOTIC CELL (after Lo et a 1, 1991A) 


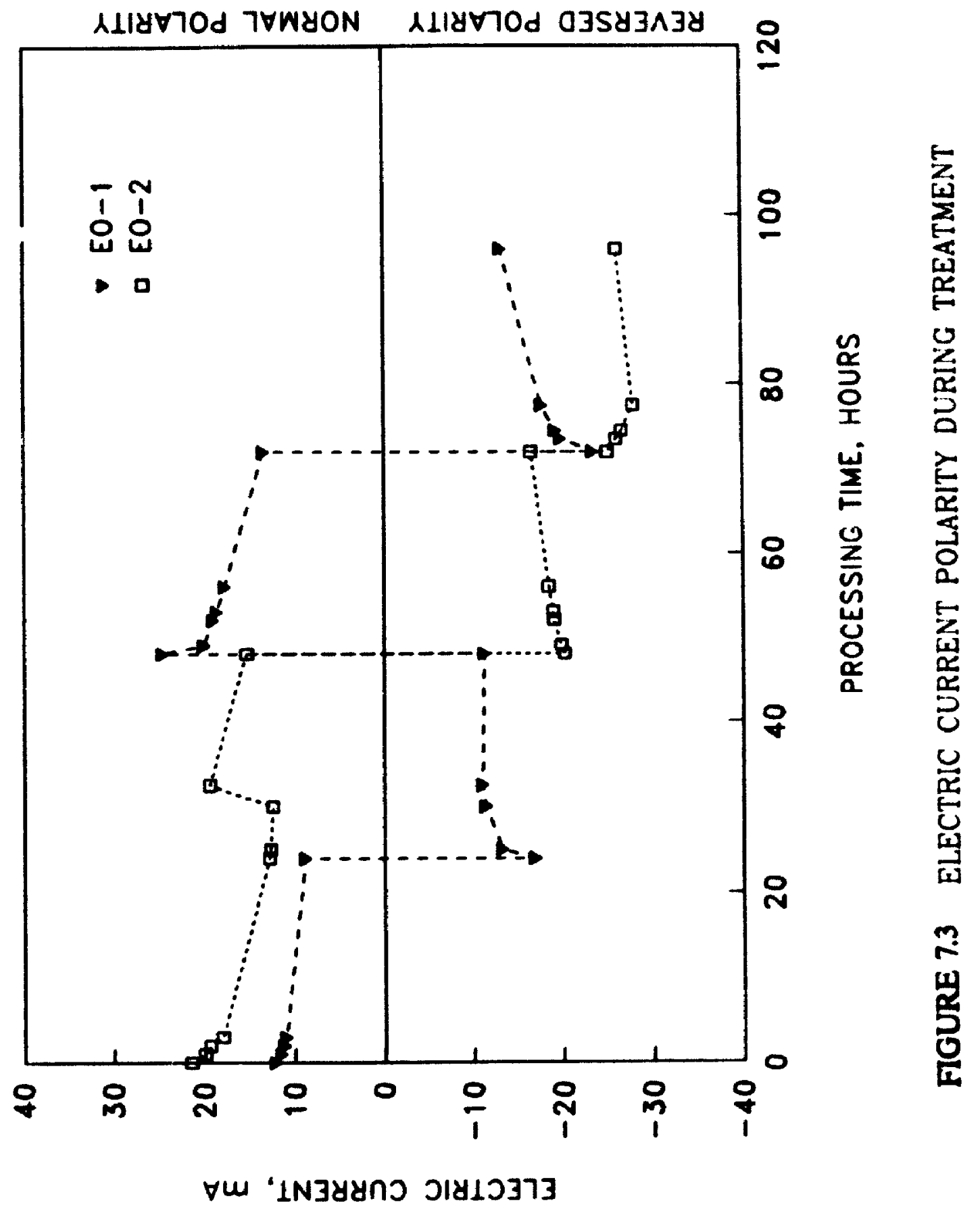




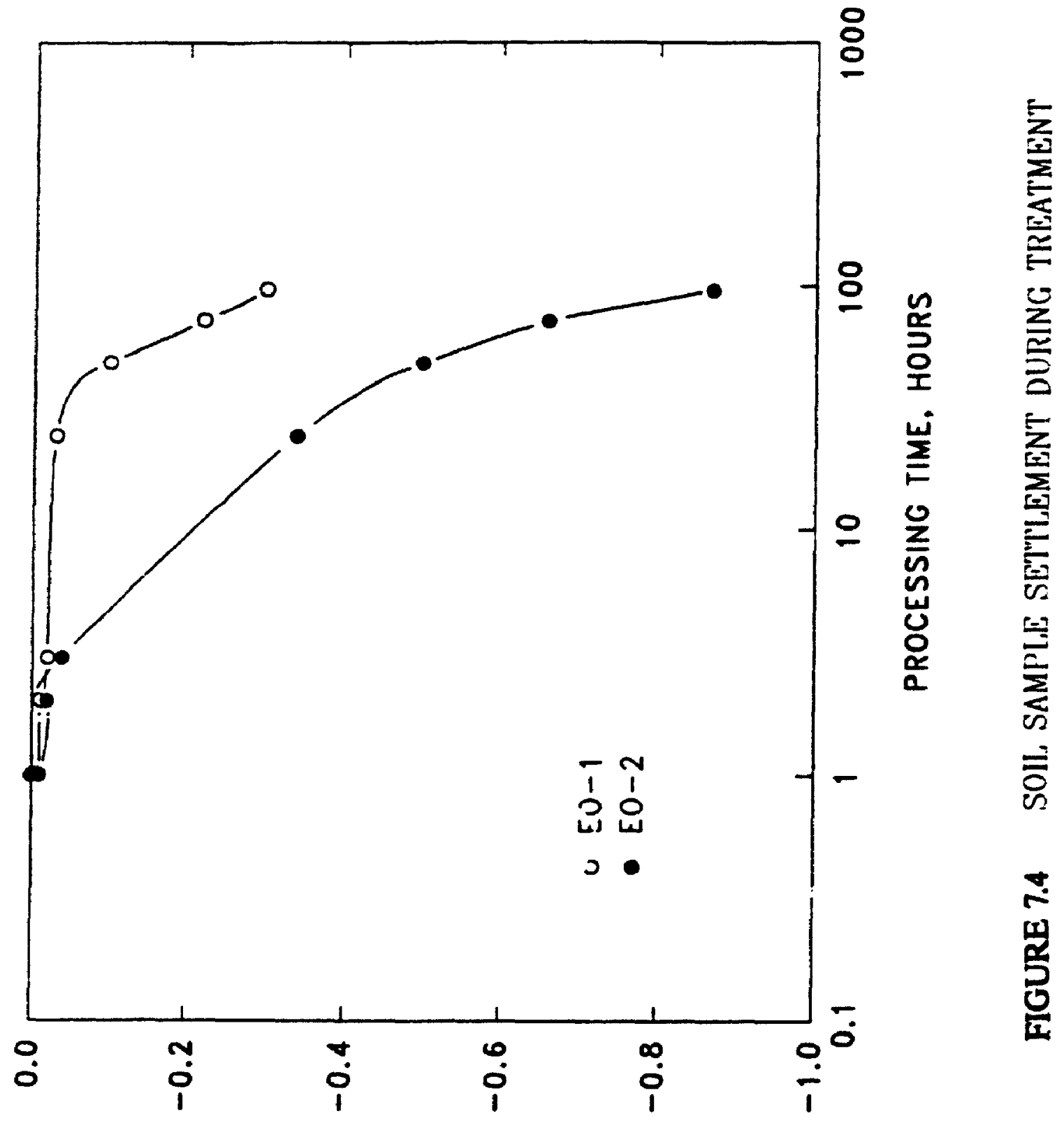

แس 'IN3W371135 


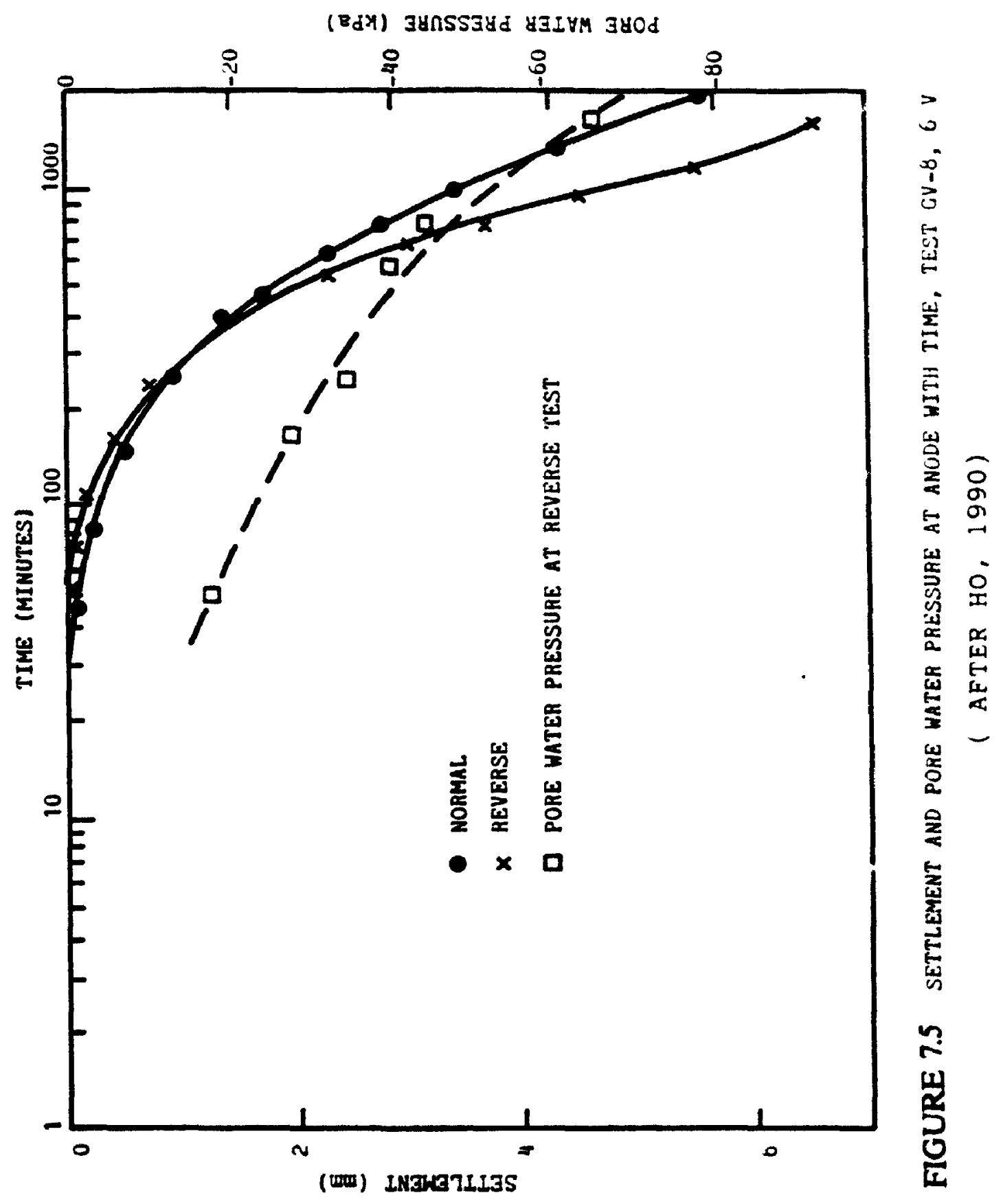




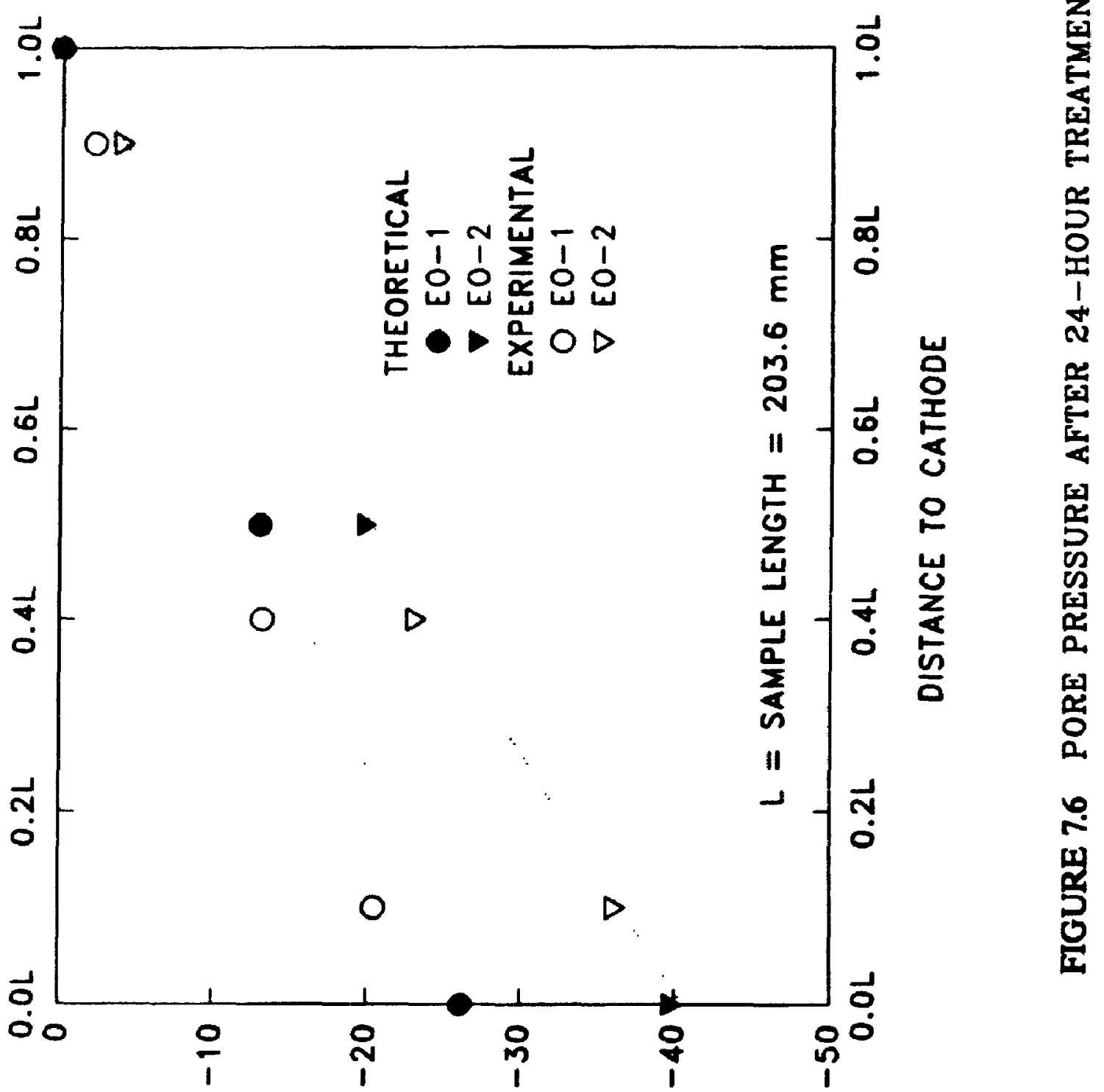

Ddy · Jynssayd jyod 


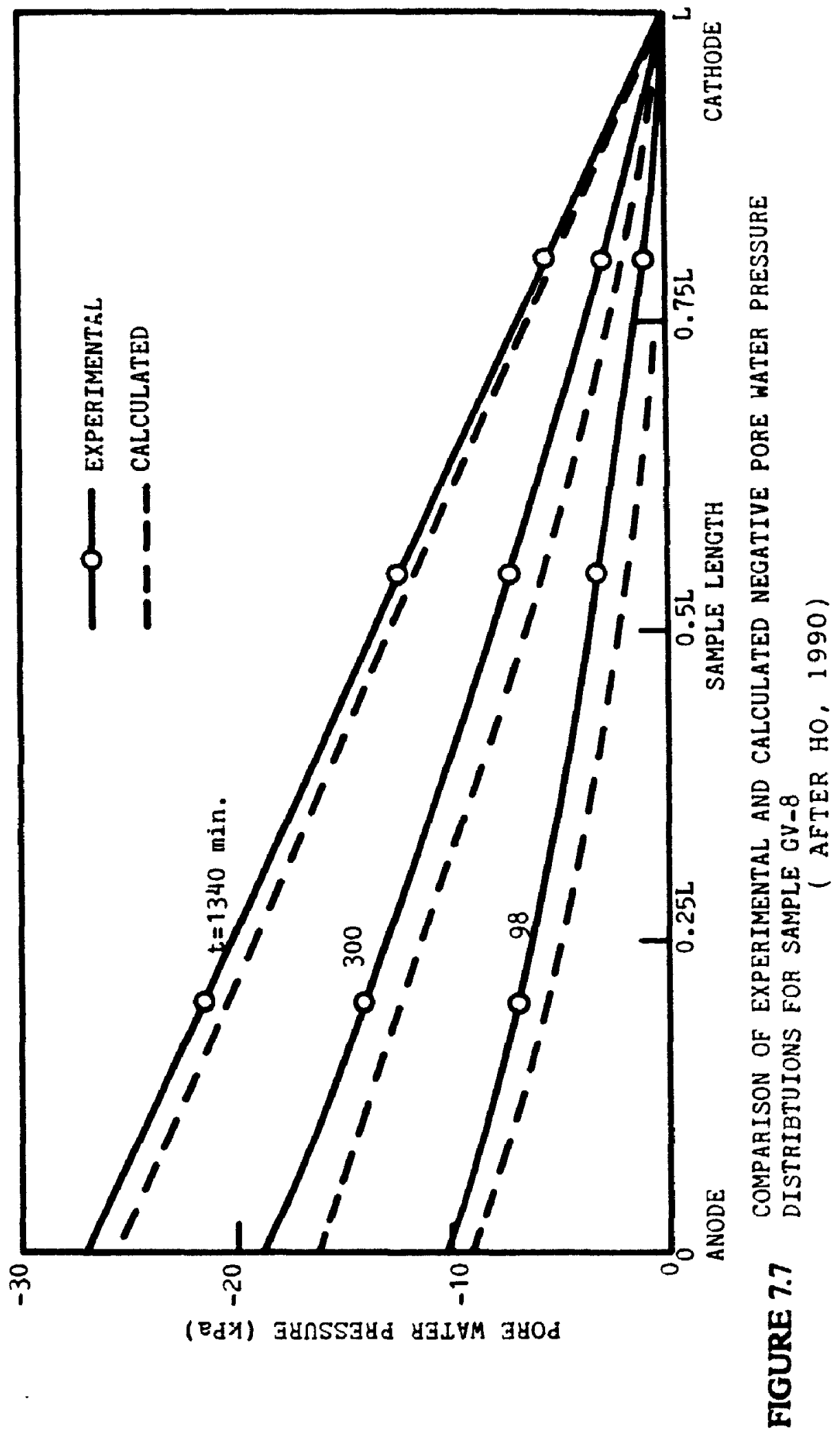




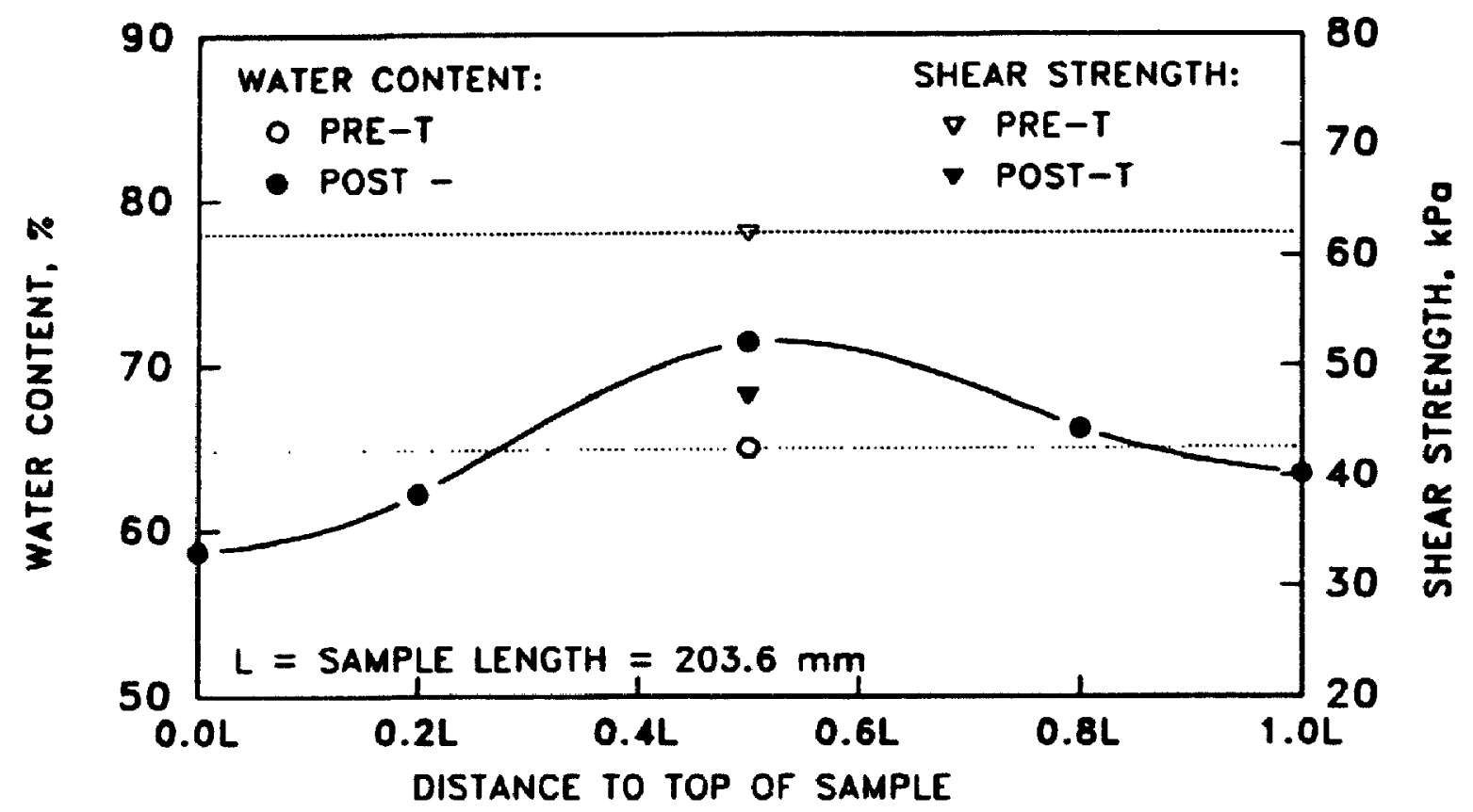

(A) EO-1

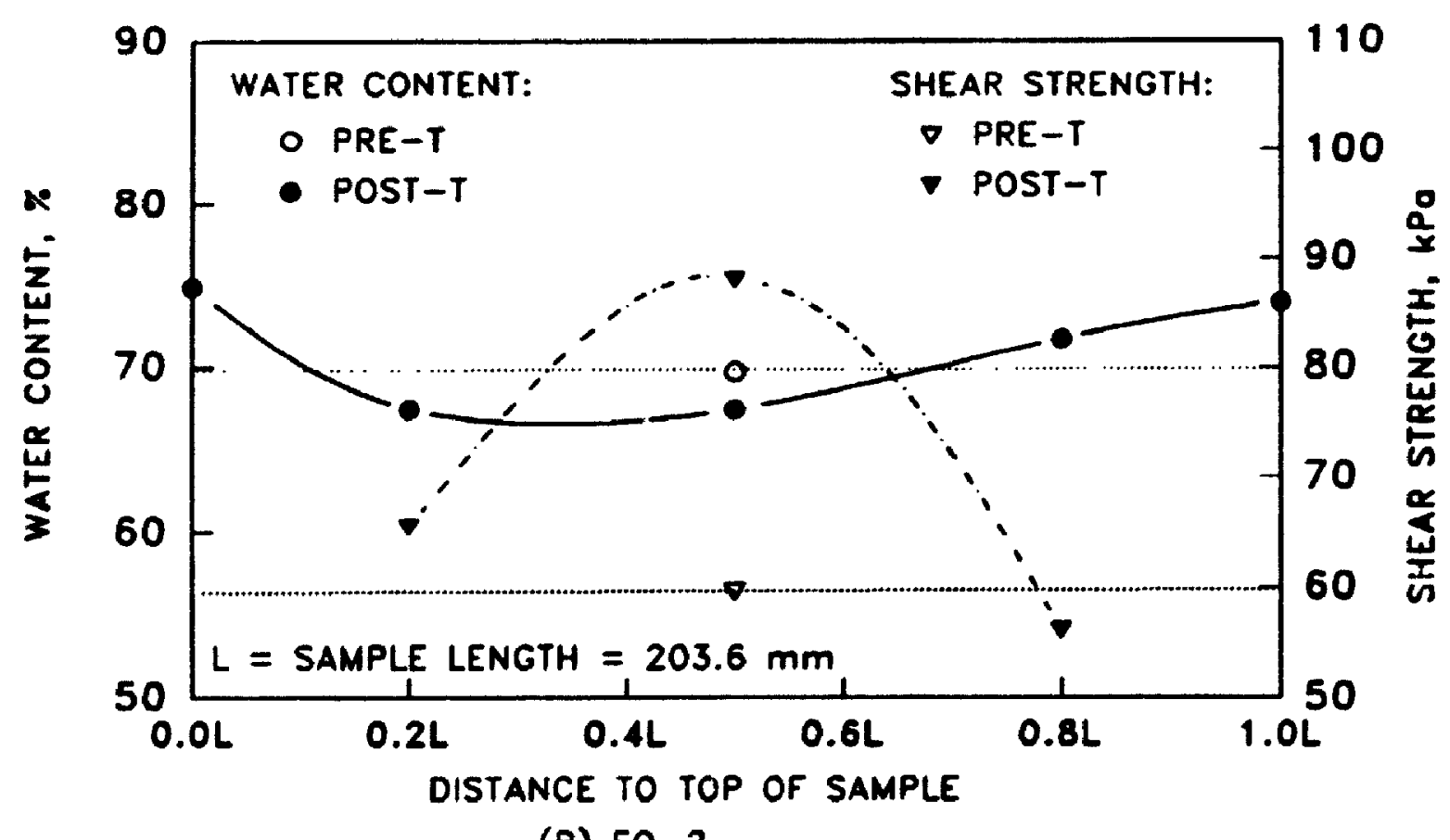

(B) EO-2

FIGURE 7.8 CHANGES OF SOIL WATER CONTENT AND SHEAR STRENGTH AFTER TREATMENT 


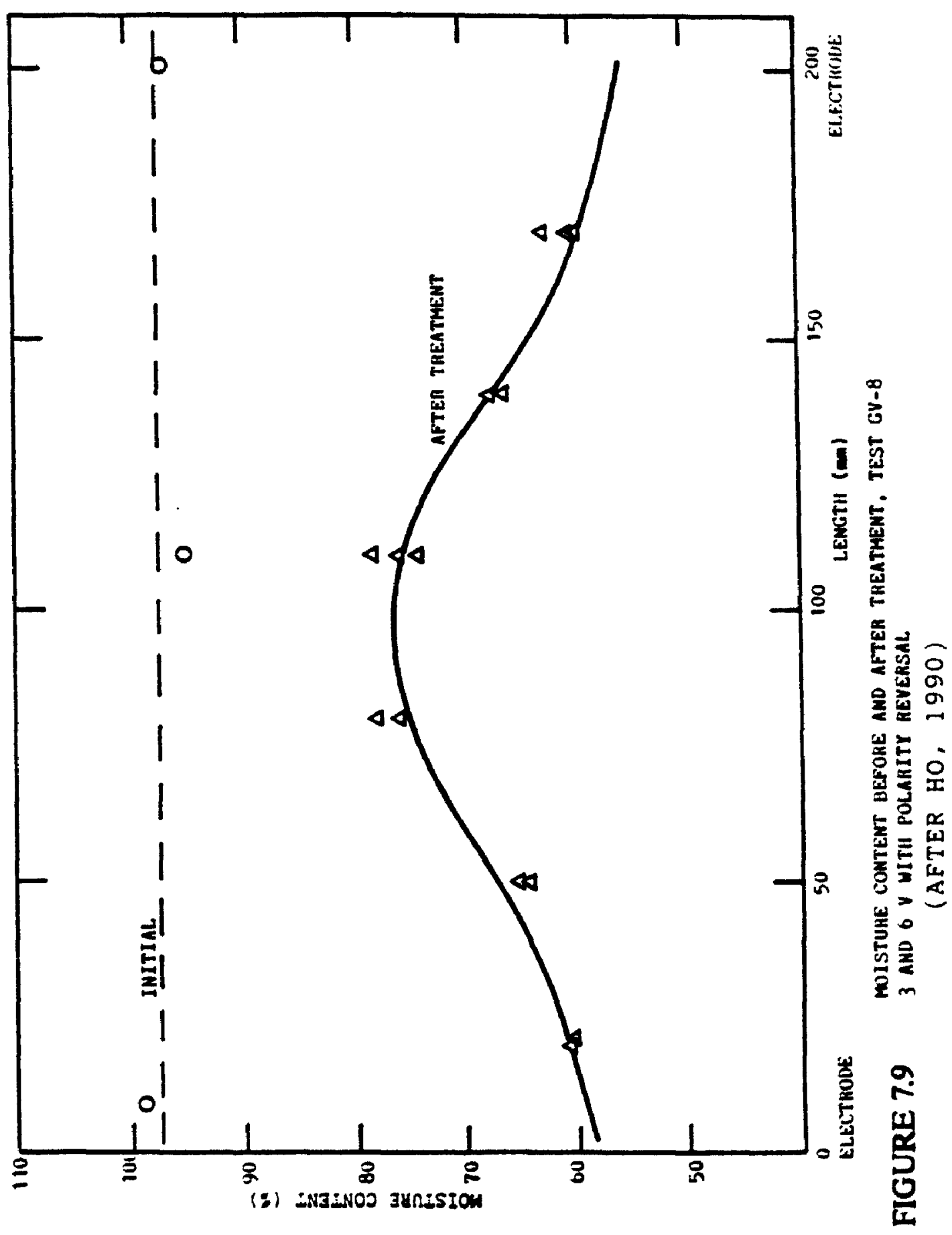




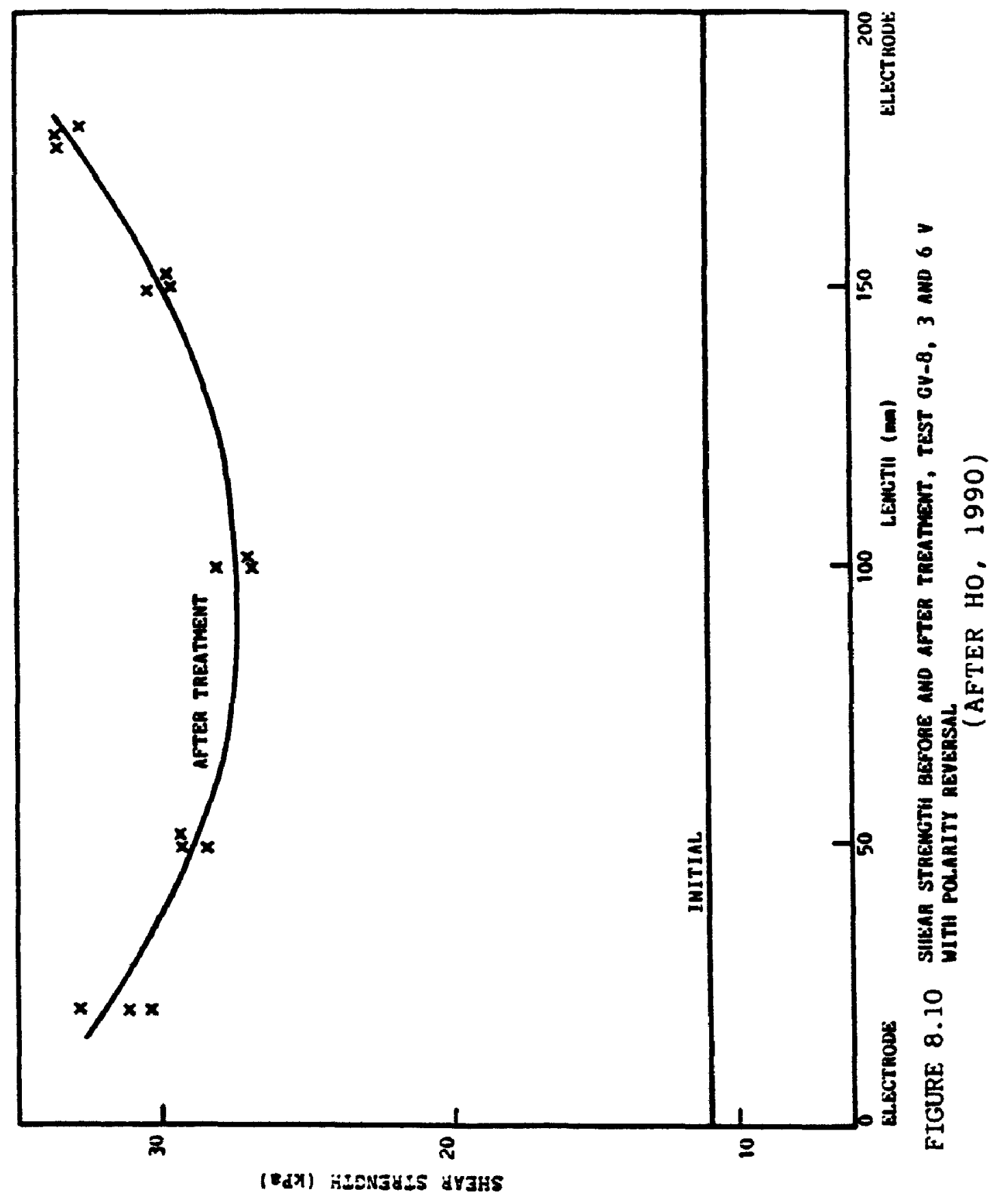




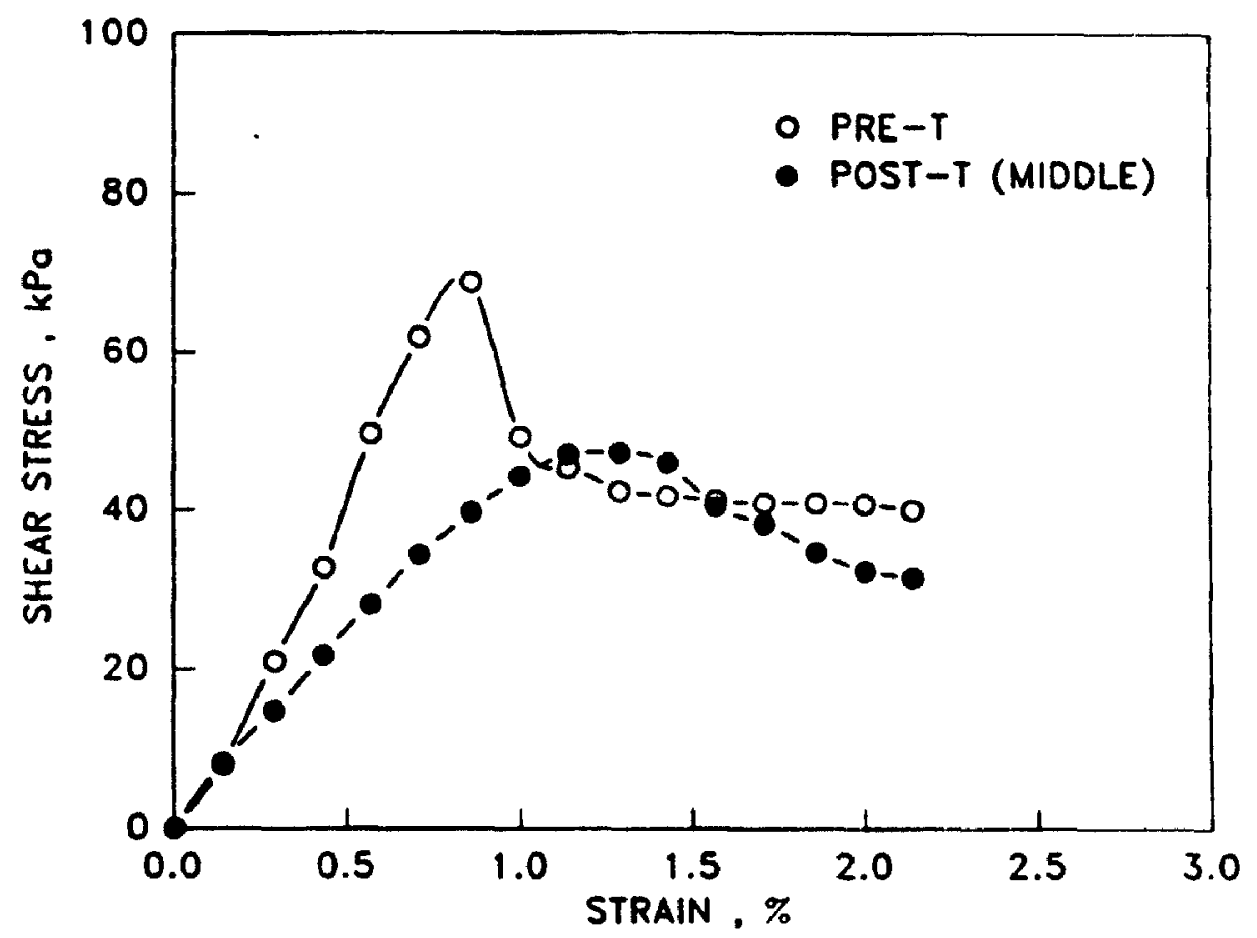

(A) TEST EO-1

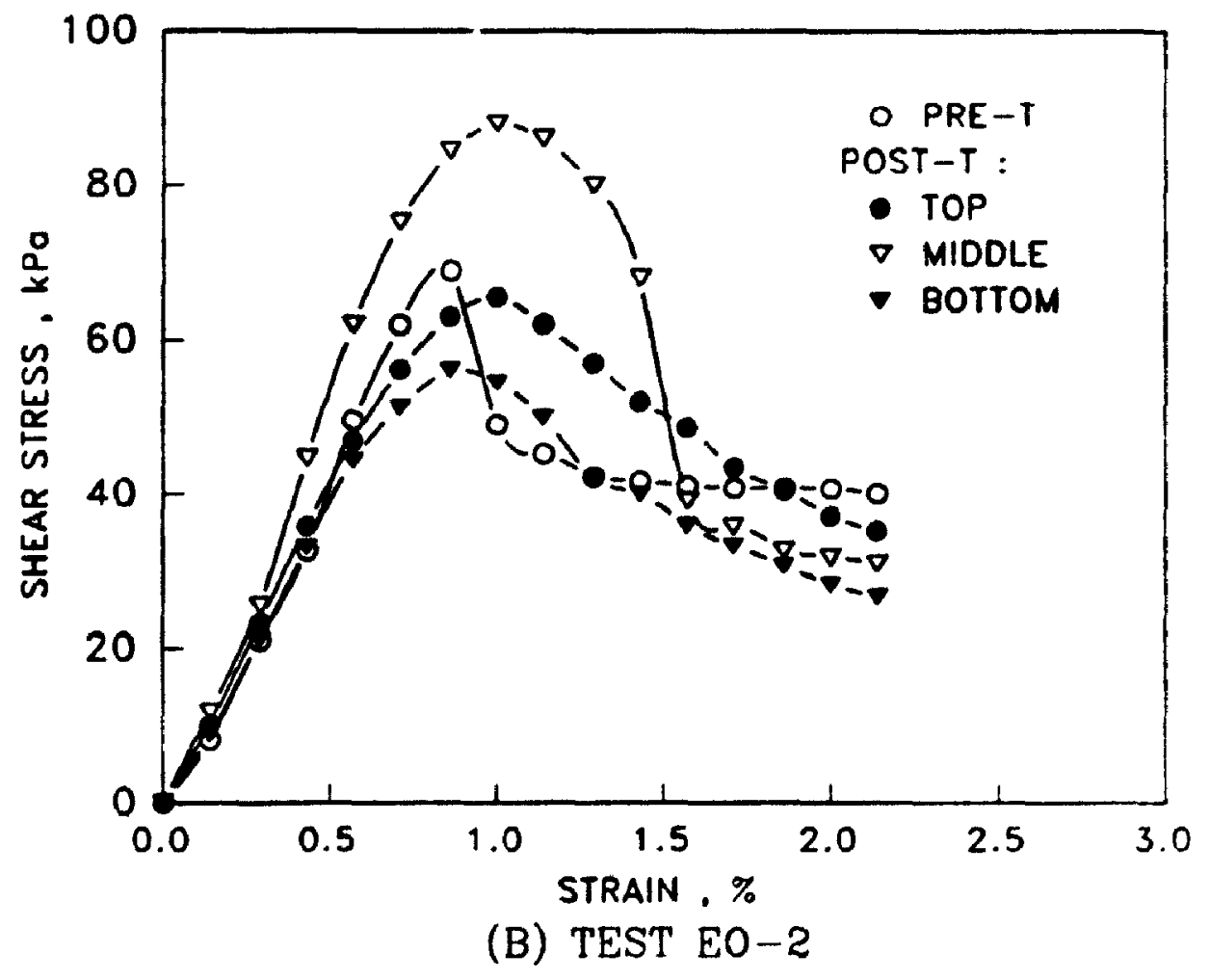




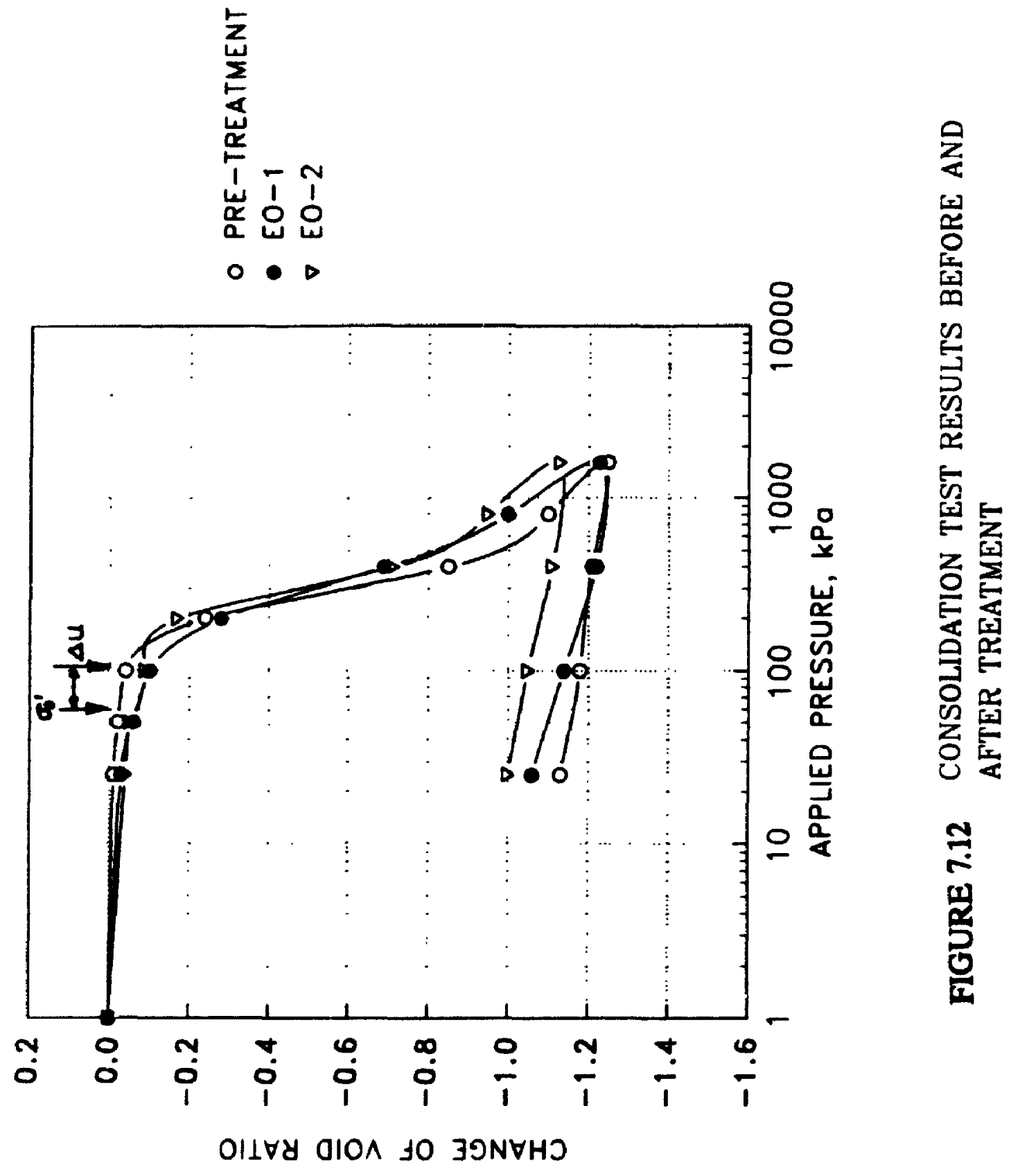




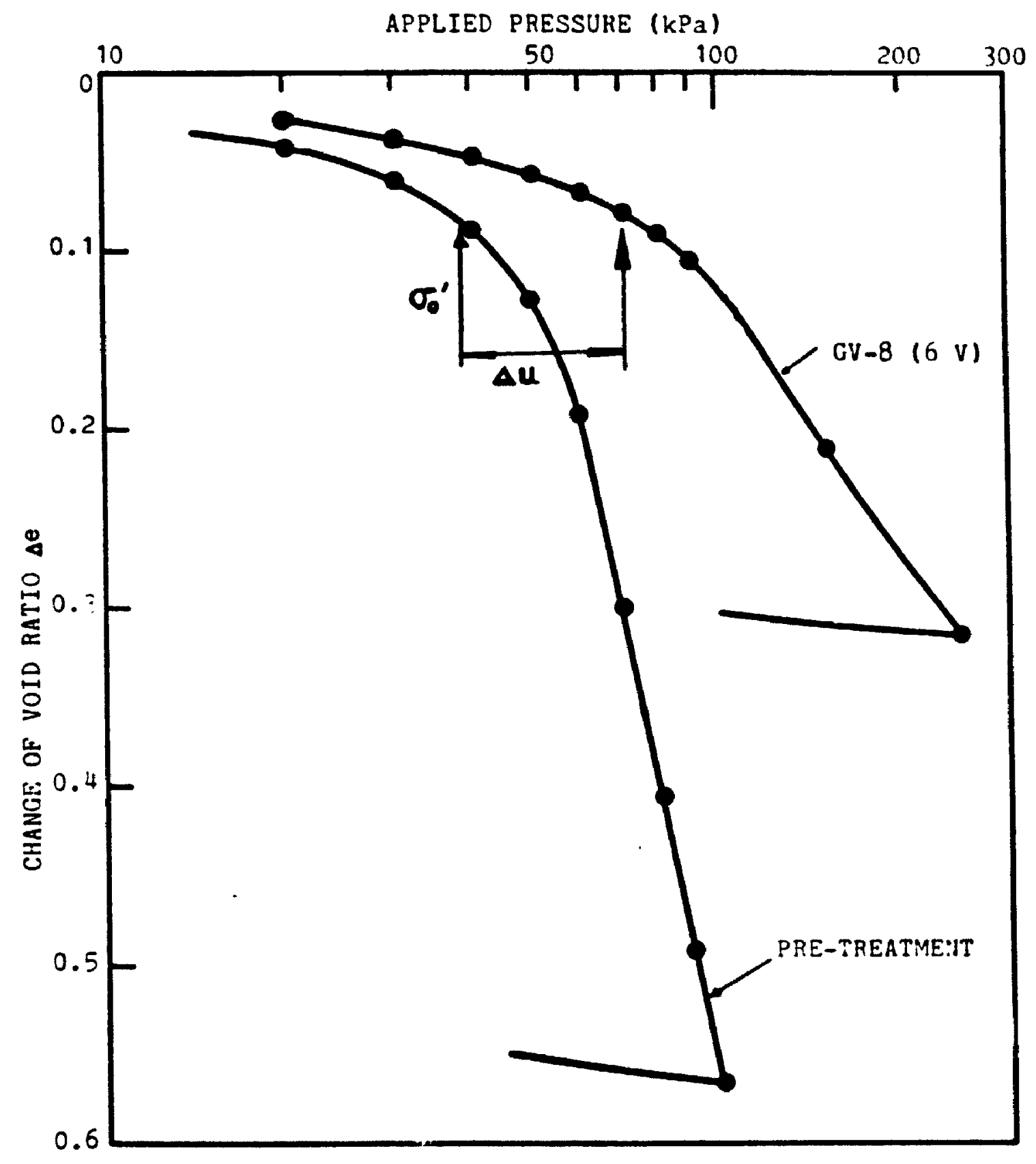

FIGURE 7.13 CONSOLIDATION CURVES FOR GLOUCESTER CLAY SAMPLES BEFORE AND AFTER TREATMENT

(AFTER HO, 1990) 
CHAPTER 8

SUMMARY, CONCLUSIONS AND RECOMMENDATIONS

FOR FURTHER RESEARCH

\subsection{Summary and Conclusions}

Strengthening of soft clays by dielectrophoresis is a new technique in geotechnical engineering. This study focused on the exploration of the principles of dielectrophoresis in clays through theoretical and experimental investigations. The behaviour of clay-water-electrolyte systems in a non-uniform electric field was analyzed and an experimental program was performed to investigate the dielectrophoretic process in natural clays. The major findings through this study can be summarized in the following subjects:

(1) The theory of dielectrophoresis in clay-water-electrolyte systems.

The dielectrophoretic force imposed on clay particles in a clay-waterelectrolyte system is controlled by the electric field configuration and the electric behaviours of the system. The non-uniform electric fields with cylindrical configurations were discussed and the analytical solutions of cylindrical electric fields were derived. The concept of equi-intensity lines was introduced to describe the magnitude and gradient of non-uniform electric fielda. The phenomenon of electrical double layer polarization was discussed and the apparent permittivities of clay-waterelectrolyte systems were quantitatively computed. The concept of effective polarizability of clay-water-electrolyte systems was introduced based on the electric properties of clay-water-electrolyte systems, including the effect of electrical double 
layer polarization. The effective polarizability provided interpretation to both magnitude and direction of dielectrophoretic force in clay-water-electrolyte systems.

Some of the most important conclusions are summarized below:

(a). The dielectrophoretic force is applied on clay particles with surrounding electrical double layer;

(b). The dielectrophoretic force is determined by the volume of particles, the intensity and gradient of electric field, and the polarizability;

(c). For most clay-water-electrolyte systems, the direction of dielectrophoretic force is towards the lower field intensity, governed by the effective polarizability of clay systems. As a result, clay particles tend to move away from the central electrode.

(2). The experiments on natural Leda clays.

The Leda clays recovered from Ottawa, Canada were used in the experimental program to investigate the dielectrophoretic process in soils. The properties of the clays, including the mechanical properties, mineralogical properties, chemical properties and electric properties, i.e., the permittivity and conductivity were determined.

The dielectrophoretic treatment on the Gloucester clay tube samples were conducted to examine the effects of intervening media in the space between the central electrode and the soil mass. The continuity of electric field is the crucial factor for the effectiveness of treatment and the optimum intervening medium should be a clay slurry with low water content. It was found from the Gloucester clay test series that: 
(c). The preconsolidation pressure increased up to $38.6 \%$.

(3). Comparative study on electroosmosis and dielectrophoresis.

Electro-osmotic experiments on the Orleans clays were conducted as a comparative study. The characteristics of dielectrophoresis and electro-osmosis for soil improvement were discussed and compared. It was concluded that the action of electroosmosis is associated with water flow induced by the electric current in a soil mass, while that of dielectrophoresis applies a force directly on the clay particles by means of a strong electric field. Electroosmosis involves electrochemical reactions associated with electric current, including a pronounced pH gradient after treatment, whereas dielectrophoresis involved primarily physical effects in the cases of insulated electrodes.

Some of the important findings may be summarized below:

(a). The action of electroosmosis is associated with water flow in the soil mass. Both strength increase and strength decrease may be achieved, depending on the direction of water transport.

(b). The change of soil properties in electroosmosis is controlled by the negative electroosmotic pore pressure in relation to the in-situ overburden pressure and the preconsolidation pressure. To improve the soil properties signifiantly, the negative pore pressure generated should exceed the preconsolidation pressure.

\subsection{Recommendation for Further Rerearch}

The strengthening effect of dielectrophoresis on the Leda clays is 
(a). The shear strength of the clay was increased significantly by dielectrophoretic treatment under the voltage of $18 \mathrm{kV}$ for 21 days. The increase of shear strength was associated with the decrease of soil water content. The maximin increase of shear strength was $37.5 \%$, associated with a decrease of water content of $26.3 \%$ at the slurry water content of $118.5 \%$.

(b). Dielectrophoresis in clays is a progressive process, i.e., the efficiency of treatment will increase with the treatment time, attributable to the characteristics of effective polarizzbility.

The dielectrophoretic treatment on the Orleans clay block samples were conducted to explore the effects of the electric field intensity and gradient. After $\mathbf{3 8}$ days of treatment under practical electrode configurations, the soil properties were improved significantly, including the increases of shear strength, pre-consolidation pressure and shearing resistance in terms of effective stress, and the decrease of sensitivity. The distributions of soil water content and shear strength after treatment were presented by means of contour maps, through which the effect of electric field was clearly illustrated. The improvement of soil properties was associated with the intensity and gradient of the electric fiels and the increase of undrained shear strength at a specific location was proportional to the dielectrophoretic force, all of these are consistent with the theory of dielectrophoresis. In particular, it was found that

(a). The maximum overall increase of shear strength was $44 \%$ for the entire soil block after 28 days of treatment at the a voltage of $15 \mathrm{kV}$.

(b). The sensitivity of the clay reduced up to $70 \%$. 
predominant, as evidenced in this study. The process is fulfilled by the dielectrophoretic force imposed directly upon the clay particles with electrical double layer. Therefore, in soft, sensitive clays in which the bonding between particles is weak, the soil deformation is significant. The effects are illustrated as the increase of soil shear strength and decrease of soil water content, as shown in experiments on the Gloucester clay. In a stiff and less compressible clay, on the other hand, the action of dielectrophoresis causes relatively small deformation. The sustained action of dielectrophoretic force results in the increase of soil shear strength associated with a small change of water content.

With the understanding obtained in this research, further research on dielectrophoresis may consider the following aspects:

(1). A field test as the third phase of experimental exploration, following the experiments on tube samples and block samples. The electrode suitable to field application should be designed. The electric field configuration, including the voltage required for adequate electric field intensity and the arrangement of electrodes for best effectiveness of treatment, should be carefully designed.

(2). Application of dielectrophoresis in geo-environmental engirieering. The process may be valuable in solving geo-enviromnental engineering related problems, such as contamination control, soil stabilization, consolidation of mining tailings and other sediments, treatment of waste water and so on.

(3). The combined effect of dielectrophoresis and electro-osmosis. In order to utilize the advantages of the two methods, it is of value to conduct more research on combined effects of dielectrophoresis and electro-osmosis. 
(4). Further investigation to the effective polarizability $\sigma_{\text {eff }}$ including the analytical solutions and the experimental verifications. The effect of mutual dielectrophoresis should be further investigated as it contrivutes significantly under the circumstances of low frequency and large concentration of clay particles.

(5). Theory of dielectrophoretic stress induced by dielectrophoretic force in a clay mass. The overall dielectrophoretic forces on clay particles can be treated as a stress imposed on the clay skeleton arising from body forces. The derivation of the theory is being undertaken.

(6). Aspects in soil physical chemistry. The physico-chemical reactions may significantly affect the engineering properties of soils, as demonstrated in this research. Further investigation towards the interaction between clay particles under dielectrophoresis is of value. 


\section{REFERENCES}

Arulanandan,K and Mitchell, J.K. (1968) Low Frequency Dispersion of Clay-WaterElectrolyte Systems. Clays and Clay Minerals, Vol.16, 337-351.

Benguigui, L and Lin, I.J., (1982) More about Dielectrophoretic Force. J. Appl. Phys. $53,2$.

Bockris,J.O'M. and Reddy, A.K.N. (1970) Modem Electrochemistry, Pleuum Press, N.Y.

Bolt, G.H. (1957) Determination of the charge density of silicate sols. J. Phys. Chem. $61,1166$.

Bozosuk, M. and Leonards, G.A.(1972). The Gloucester test fill. Special Conference on Performance of Earth and Earth-Supported Structures, ASCE, Purdue University, Vol. 1, Part 1, 299-317.

Casagrande, L (1941) Die Elektrische Entwasserung Feinkorniger Boden. Die Strasse, No. 19-21.

Casagrande, L (1952) Electro-osmosis stabilization of soils. J. of Boston Society of Civil Engineers Vol. 39, 51-83.

Chen, K.W.L.(1967) Dielectrophoresis of Solids in Aqueous Solutions M.Sc. thesis, Oklahoma State University, U.S.A.

Clark, S. P. (Ed.) (1966) Hand Book of Physical Constants, Geological Society of America. New York.

Conlon, R.J. (1966) Land Slide on the Toulnustouc River, Quebec. Canadian Geotechnical Joumal, 3, 113-114.

Crawford, C.B. (1961) Engineering Studies of Leda Clay. Soils in Canada. The Royal Society of Canada, Ed. R.F.Legget, 200-217.

Dukhin, S. S. and Shilov, V. N. (1974) Dielectric Phenomena and Double Layer in Disperse Systems and Polyelectrolytes, John Wiley and Sons, Inc. New York

Esrig, M.I. (1968) Pore pressures, consolidation and electrokinetics. ASCE Journal of the Soil Mechanics and Foundations Division, 94(SM4):899-302.

Hill, N. E., Vaughan, W., Price, A.H. and Davies, M. (Ed) (1969) Dielectric Properties and Molecular Behaviour. Van Nostrand Reinhold Co. Ltd. 
Ho, KS. (1990) Electrical Strengthening of Soft Sensitive Clays. Ph.D. Thesis, University of Western Ontario, Canada.

Iler, R.K (1970), Surface Colloid Sci, 6,1.

Inculet, I.I. and Lo,.KY. (1988). Dielectric Consolidation of Clays. Proceedings of 23rd Conference, Industry Applications Society, Institute of Electrical and Electronic Engineers, Pittsburgh, October.

Inoue, T., Pethig, R, Al-Ameen, T.A.K, Burt, J.P.H. and Price, J.A.R. (1988) Dielectrophoretic Behaviour of Micrococcus Lysodeilticus and its Protoplast. J. Electrost. 21, 215-223

James, R.O. and Parks, G.A (1980) Characterization of aqueous colloids by their electrica: double layer and intrinsic surface chemical properties. Surf. Colloid Sci, 29, 119.

Jones, T. B. (1979) Dielectrophoretic Force Calculations. J. Electrost. 69-82

Jones, T.B. and Kallio, G.A. (1979) Dielectrophoretic Levitation of Spheres and Shells. J. Electrost. 6, 207.

Karrow, P.F. (1961) The Champlain Sea Clay and Its Sediments. Soils in Canada. The Royal Society of Canada, Edited by R.F.Legget, 97 - 108.

Kaye, G.W.G. and Laby, T.H. (Ed.) (1986) Tables of Physical and Chemical Constants. John Wiley and Sons Inc. New York.

Kenney, T.C., (1968) A review of recent research on strength and consolidation of soft sensitive clays. Canadian Geotechnical Joumal, 5, 97-119.

Kraus, J.D. and Carver K R. (1973) Electromagnetics. McGraw-Hill.

Lee, I.K (1968) (Ed.) Soil Mechanics - Selected Topics. American Elsevier, N.Y.

Leonar ds, G.A and Ramiah, B.K (1960) Time Effects in the Consolidation of Clays. ASTM Special Technical Publication 254, 116-130.

Linsay, W.L (1979) Chemical Equilibria in Soils. John Weley \& Sons, Inc.

Lo, KY., Bozosuk, M. and Law, KT. (1976) Settlement analysis of the Gloucester test fill. Can. Geo. J. Vol.13. 339-354.

Lo, K.Y., Inculet, I.I., and Ho, K.S. (1991a) Electroosmotic strengthening of soft sensitive clays Canadian Geotechnical Joumal Vol.28, No.1, 62 - 73. 
Lo, K.Y., Inculet, I.I., and Fio, K.S. (1991b) Field test of electroosmotic strengthening of soft sensitive clay Canadian Geotechnical Joumal Vol.28, No.1, 7483.

Lo, KY., Ho,K.S. and Inculet,I.I. (1992) A Novel Technique of Electrical Strengthening of Soft Sensitive Clays by Dielectrophoresis. Canadian Geotechnical Joumal Vol.29, No.4.

Lockhart, N.C. (1983) Dielectrophoretic Consolidation of Clays. J. Electrost. 10, 17-22.

McEuen, P. B., (1966) Clays and Clay Minerals, 14:549

Mitchell, J.K (1976) Fundamentals of Soil Behaviour. John Wiley \& Sons, New York.

Mitchell, J.K. (1991) Conduction phenomena: from theory to geotechnical practice. Geotechnique. 41, No.3, 299-340.

Newman, A.C.D. (1987) Chemistry of Clays and Clay Minerals. Longman Sciences and Technology. Mineralogical Society, London, England.

Van Olphen, H. (1977) An Introduction to Clay Colloid Chemistry, Interscience, N.Y.

Pohl, H. A, (1951) The motion and precipitation of suspensoids in divergent electric fields. J. Appl. Phys. , 22:869

Pohl, H. A and Schwar, J.P. (1959) Factors affecting separation of suspensions in nonuniform electric fields. J. Appl. Phys. , 30-69

Pohl, H. A, (1978) Dielectrophoresis, Cambridge University Press

Quigley, R.M. (1980) Geology, mineralogy and geochemistry of Canadian soft soils: a geotechnical perspective Canadian Geo. J. Vol.20, No.2, 288-298.

Saito, M. and Schwan, H.P. (1960) Biological Effects of Microwave Radiation, Vol. 1, 85, Ed. M.F. Peyton, Plenuum Press, New York.

Santamaria, C., Iglesias,F.J., and Domingues, A (1985) Dielectrophoretic deposition in suspensions of macromolecules: polyvinylchloride and sephadex G50. J. Colloid \& Interface Sci, Vol. 103, No.2, 508-515

Schwan, H.P.(1957) Physical Techniques in Biological Research. W.L.Nastuk, Ed. 1, Academic Press, N.Y.

Schwarz, G. (1962) The theory of the low-frequency dielectric dispersion of colloidal particles in electrolyte solution. J. Phys. Chem. Vol.66, 2636-2642. 
Scrimager, C. Dielectrophoresis of Inorganic Colloidal Suspensions (1974), M.Sc. thesis, OkJahoma State University, U.S.A.

Shilov, V. N. and Estrela-L'opis V.R. (1975) Theory of movement of spherical particles of a suspension in an inhomogeneous electric field. Research in Surface Forces, Consultants Bureau, New York, Vol. 4, 121.

Shilov, V.N. and Rozen, Y.Y. (1975) Polarization of Diffuse Double Layer of RodShaped Particles and Giant Low-frequency Dielectric Constant of Polyelectrolytes. Research in Surface Forces, (Ed. Deryagin) Consultants Bureau, N.Y. Vol.4, 108-120.

Smoluckowski, M. (1914). Handbuch der Elektrizitat und Magnetismus. Edited by L. Graetz, J.A. Barth, Vol. 2

Soderman,LG. and Quigley, R.M. (1965) Geotechnical Properties of Three Ontario Soils Can.Geo.J. Vol.2 No.2, 176-189.

Sparks, D.L(Ed.) (1986) Soil Physical Chemistry, CRC Press, Florida.

Stoylov, S and Petkanchin, I., (1975) Movement of Spherical Colloidal Particles in An Inhomogeneous Electric Field. Research in Surface Fonces, Consultants Bureau, New York, Vol. 4, 138.

Townsend, D.L, Sangrey, D.A, and Walker, LK (1969). The brittle behaviour of natural cemented soils. Proceedings, 7th International Conference on Soil Mechanics and Foundations Engineering, Mexico City, Vol. 2, 411-417.

Verwey, E.J.W., (1935) Chemical Review, 16, 393.

Vorobeva, T.A, Vlodavets, I.N. and Zubov, P.I.(1969) Kolloidnyi Zhumal, Vol. 31, No.5, 668-673

Winslow, W.M.(1949) Induced fibration of suspensions. J. Appl. Phys., 20:1137

Yong, R.N., Sethi, A.J., and La Rochelle, P. (1979) Significance of amorphous material relative to sensitivity in some Champlain clays. Canadian Geotechnical Joumal, 16, 511-520. 


\section{APPENDIX 1 \\ DISTRIBUTION OF CYLINDRICAL ELECTRIC FIELD}

\section{A1.1. Perfect Cylindrical Geometry}

The distribution of electric field intensity with perfect cylindrical symmetry as shown in Fig.3.1 can be derived as the follows:

Consider a cylindrical electric field consisted of a central electrode with a metallic rod of radius $R_{a}$ insulation layer of radius $R_{b}$ and permittivity $\epsilon_{b}$, and an outer electrode of radius $R_{c}$ The annular space between the central and outer electrode is filled with a material of permittivity $\epsilon_{\mathrm{m}}$. The perfect cylindrical symmetry is only valid when the length of the electrodes, 1 , is much larger than the separation distance, $R_{c}-R_{y}$, between the central and outer electrode.

Suppose an ac voltage $U_{0}$ is applied on the central electrode and the outer electrode is grounded. The electric field intensity distribution can be represented in the cylindrical coordinates as

$$
E=\left\{\begin{aligned}
0 & \left(0 \leq r \leq R_{a}\right) \\
\frac{\rho_{L} r_{0}}{2 \pi \epsilon_{b} r} & \left(R_{a} \leq r<R_{b}\right) \\
\frac{\rho_{L} r_{0}}{2 \pi \epsilon_{m} r} & \left(R_{b} \leq r<R_{c}\right) \\
0 & \left(r \geq R_{c}\right)
\end{aligned}\right.
$$

where

E: electric field intensity (vector), (volt/m) 
r: distance to the centre of coordinates, $(\mathrm{m})$ :

$\mathbf{r}_{\mathbf{0}}$ : unit radial vector;

$\rho_{L}:$ linear charge density, $(\mathrm{C} / \mathrm{m})$;

$\epsilon_{\mathrm{b}}$ : permittivity of insulator, $(\mathrm{F} / \mathrm{m})$;

$\epsilon_{m}$ : permittivity of the material filled in the annular space between $R_{b}$ and $R_{c}$ $(\mathrm{F} / \mathrm{m})$.

According to electromagnetics, the potential difference between $\mathbf{R}_{\mathbf{a}}$ and $\mathbf{R}_{\mathrm{c}}$ is

$$
U_{0}=\int_{R_{e}}^{R_{c}} E \cdot d r
$$

Substitute (A1.1) into (A1.2) and integrate

$$
\begin{aligned}
U_{0} & =\frac{\rho_{L}}{2 \pi}\left(\frac{1}{\epsilon_{b}} \ln \frac{R_{b}}{R_{a}}+\frac{1}{\epsilon_{m}} \ln \frac{R_{c}}{R_{b}}\right) \\
& =\frac{\rho_{L} \lambda}{2 \pi}
\end{aligned}
$$

where

$$
\lambda=\frac{1}{\epsilon_{b}} \ln \frac{R_{b}}{R_{a}}+\frac{1}{\epsilon_{m}} \ln \frac{R_{c}}{R_{b}}
$$

The linear charge density $\rho_{L}$ can be obtained from (A1.3)(Eq.(3.2) in the thesis) as

$$
\rho_{L}=\frac{2 \pi U_{0}}{\lambda} \quad(\text { coulomb/m) }
$$


The charge density is proportional to the electric field intensity, Ec.(A1.1).

Substitute (A1.5) into (A1.1), we obtain the electric field intensity distribution in terms of the potential difference $U_{0}$ :

$$
E=\left\{\begin{aligned}
0 & \left(0 \leq r<R_{a}\right) \\
\frac{U_{0} r_{0}}{\epsilon_{b} \lambda r} & \left(R_{a} \leq r<R_{b}\right) \\
\frac{U_{0} r_{0}}{\epsilon_{m} \lambda r} & \left(R_{b} \leq r<R_{c}\right) \\
0 & \left(r \geq R_{c}\right)
\end{aligned}\right.
$$

Eq.(A1.6) is the electric field intensity in a perfect cylindrical geometry (Eq.(3.1) in the thesis).

\section{A1.2. Electrode-Pair}

As shown in Fig.3.3, an electrode pair is readily constituted by two electrodes arranged in parallel. The centre-to-centre distance between the electrodes is $2 \mathrm{~s}$. A fixed electric potential difference $U_{0}$ is maintained between the electrodes thus the charge on the electrodes is equal in magnitude but apposite in sign. The magnitude of the linear charge density $p_{L}$ on each electrode can be calculated from Eq.(A1.5), i.e.,

$$
\rho_{l}=\frac{2 \pi\left(\frac{U_{0}}{2}\right)}{\lambda}=\frac{\pi U_{0}}{\lambda}
$$


Note that the potential difference used to calculate the charge density for each of the two electrodes is $1 / 2 U_{0}$ because of the symmetry of the electrode pair, Fig.3.3.

The parameter $\lambda$ is obtained from Eq.(A1.4) in which the radius of the outer electrode $\mathbf{R}_{\mathbf{c}}$ is taken as the half distance between the electrodes, $\mathbf{s}$. It should be noted that the charge is not uniformly distributed on the electrode conductor surface but has higher density on the mutually facing sides of the conductors.

Let the origin of the coordinates be the reference for potential, as shown in Fig.3.3. According to the principles of electromagnetics, the potential difference due to two electrodes of opposite signs between $P(x, y)$ and the origin of coordinates is

$$
U=\frac{P_{L}}{4 \pi e_{m}} \ln \frac{(x+s)^{2}+y^{2}}{(x-s)^{2}+y^{2}}
$$

Substitute Eq.(A1.7) into (A1.8),

$$
U=\frac{U_{0}}{4 \lambda e_{m}} \ln \frac{(x+s)^{2}+y^{2}}{(x-s)^{2}+y^{2}}
$$

where

$$
\begin{aligned}
& U_{0}=\text { potential difference between the electrode-pair; } \\
& \epsilon_{m}=\text { permittivity of material in the electric field; } \\
& A=\text { parameter determined by Eq.(A1.4) in which } R_{c}=s .
\end{aligned}
$$

The potential and electric field intensity is related by the equation

$$
\boldsymbol{E}=-\boldsymbol{\nabla} \boldsymbol{U}
$$

The electric field intensity is the gradient of potential in Eq.(A1.10), 


$$
\begin{aligned}
E & =-\nabla U=-\left(\frac{\partial U}{\partial x} I+\frac{\partial U}{\partial y} J\right) \\
& =E_{x} I+E_{y} J
\end{aligned}
$$

where $\mathbf{i}$ and $\mathbf{j}$ are unit vectors on $\mathbf{x}$ and $\mathbf{y}$ directions, respectively.

Calculate $E_{x}$ and $E_{\gamma}$ in Eq.(A1.11) through partial differentiation of the potential $U$ in Eq.(A1.8),

$$
\begin{aligned}
E_{x} & =-\frac{\partial U}{\partial x} \\
& =-\frac{U_{0}}{2 \lambda \epsilon_{m}}\left[\frac{x+s}{(x+s)^{2}+y^{2}}-\frac{x-s}{(x-s)^{-2}+y^{2}}\right] \\
E_{y} & =-\frac{\partial U}{\partial y} \\
& =-\frac{U_{0} y}{2 \lambda \epsilon_{e}}\left[\frac{1}{(x+s)^{2}+y^{2}}-\frac{1}{(x-s)^{2}+y^{2}}\right]
\end{aligned}
$$

The absolute value of the electric field intensity is

$$
|E|=\sqrt{E_{x}^{2}+E_{y}^{2}}
$$

where $E_{\alpha}$ and $E_{\gamma}$ are given by (A1.12) and (A1.13) respectively. 
A1.3. Electrode-Matrix

Electrode-matrix is another convenient way to create a non-uniform electric field. The arrangement of electrode can be rather arbitrary, as long as the distances between electrodes are much less than the length of electrodes so the assumption of infinite length electrode is valid. An example of the electrode-matrix is a central electrode with higher potential surrounded by four electrodes of equal potential at lower value, as shown in Fig.3.5. The potential differince between the central electrode and any of the surrounding electrode is $U_{\sigma}$. Similar to the electrode-pair, the linear charge density on each of the electrodes is contributed by the fixed potential difference. Let the reference for potential be at infinity, then the potential difference between a point $P(x, y)$ and infinity is

$$
U=-\frac{U_{0}}{4 \lambda \epsilon_{m}} \ln \left(\frac{r_{0} \cdot r_{-}^{3}}{r_{1} \cdot r_{2} \cdot r_{3} \cdot r_{4}}\right)^{2}
$$

where

$\mathrm{U}_{\mathrm{o}}=$ potential difference between the central and surrounding electrodes;

$\lambda=$ parameter determined from Eq.(A1.4) with $R_{c}=s ;$

$\epsilon_{\mathrm{m}}=$ permittivity of medium;

$r_{0}, r_{1}, I_{2}, r_{3}, I_{4}=$ distances from $P(x, y)$ to the electrodes, respectively, as shown in Fig.3.5:

$$
\begin{aligned}
& \mathbf{r}_{0}=\left(x^{2}+y^{2}\right)^{1 / 2} ; \\
& \mathbf{r}_{1}=\left[(x+d)^{2}+(y-d)^{2}\right]^{/ / 2} ; \\
& \mathbf{r}_{2}=\left[(x+d)^{2}+(y+d)^{2}\right]^{/ /} ;
\end{aligned}
$$




$$
\begin{aligned}
& r_{3}=\left[(x-d)^{2}+(y+d)^{2}\right]^{k_{2}} ; \\
& r_{4}=\left[(x-d)^{2}+(y-d)^{2}\right]^{/ / 2} ; \\
& r_{-} \rightarrow \infty \text {, distance to the potential reference; }
\end{aligned}
$$

$d=s / \sqrt{2}, s=$ distance between the central and surrounding electrodes, Fig.3.5. Calculate the electric field intensity from Eq.(A1.10), we obtain $E_{x}$ and $E_{\gamma}$ along $x$ and $y$ directions, respectively,

$$
\begin{aligned}
E_{x} & =-\frac{\partial U}{\partial x} \\
& =\frac{U_{0}}{2 \lambda \epsilon_{m}}\left(\frac{x}{r_{0}^{2}}-\frac{x+d}{r_{1}^{2}}-\frac{x+d}{r_{2}^{2}}-\frac{x-d}{r_{3}^{2}}-\frac{x-d}{r_{4}^{2}}\right) \\
E_{y} & =-\frac{\partial U}{\partial y} \\
& =\frac{U}{2 \lambda \epsilon_{m}}\left(\frac{y}{r_{0}^{2}}-\frac{y-d}{r_{1}^{2}}-\frac{y+d}{r_{2}^{2}}-\frac{y+d}{r_{3}^{2}}-\frac{y-d}{r_{4}^{2}}\right)
\end{aligned}
$$

Note that the distance to the potential reference is $r_{-} \rightarrow \infty$, which is treated as a constant in the differentials.

The absolute value of the electric field intensity is then

$$
|E|=\sqrt{E_{x}^{2}+E_{y}^{2}}
$$

where $E_{x}$ and $E_{y}$ are derived from $E_{q} .(A 1.16)$ and $E q .(A 1.17)$, respectively. 


\section{APPENDIX 2}

\section{EXAMPLE CALCULATION OF A KAOLINITE-WATER-ELECTROLYTE SYSTEM}

\section{Stern Potential and Double Layer Charges}

By applying equations (3.13), (3.14), (3.15) and (3.20) and utilizing the data from the literature (Mitchell, 1976; van Olphen, 1977) and Table 3.1c, the Stern potential and double layer charges may be computed.

Consider the kaolinite-water-electrolyte system discussed in Section 3.3.2. The typical cation exchange rapacity (CEC) of kaolinite is: (Mitchell, 1976)

$\mathrm{CEC} \approx 5 \mathrm{meq} / 100 \mathrm{~g}$ soil $=0.05 \mathrm{meq} / \mathrm{g}$ soil $\times 96.5 \mathrm{C} / \mathrm{g}=4.825 \mathrm{C} / \mathrm{g}$

The typical specific surface of kaolinite is (Mitchell, 1976)

$$
\mathrm{S}=15 \mathrm{~m}^{2} / \mathrm{g}
$$

The negative surface charge, therefore, is

$$
\rho=-\mathrm{CEC} / \mathrm{S}=-0.322 \mathrm{C} / \mathrm{m}^{2}
$$

The other parameters are known as follows:

(2) $N_{i}=10^{19} / \mathrm{m}^{2}$, assuming that each site occupied by a water molecule in a monolayer is a potential cation side (van Olphen, 1977).

(3) $\mathrm{M}=0.0585 \mathrm{~kg}$, molar mass of solvent $(\mathrm{NaCl})$, Table $3.1 \mathrm{c}$.

(4) $\quad v=1000 \mathrm{~kg} / \mathrm{m}^{3}$, density of water.

(5) $c=0.01 \mathrm{~N}=10 \mathrm{~mol} / \mathrm{m}^{3}$, I lar concentration of electrolyte, Table 3.1c.

(6) assuming $\Phi=0$ for simplified case (van Olphen, 1977).

(7) $d=0.5 \mathrm{~nm}$, two monomolecular layers of adsorbed water (van Olphen, 1977)

(8) $z=1$, valence of $\mathrm{Na}^{+}$, Table 3.1c. 
(9) $\quad \mathrm{T}=293 \mathrm{~K}$, absolute temperature $\left(70^{\circ} \mathrm{F}\right.$, Table $\left.3.1 \mathrm{c}\right)$.

(10) $K_{d}=\epsilon_{d} / \epsilon_{0}=6$, permittivity of water in the Stern layer (van Olphen, 1977). Introducing

$$
y_{d}=\frac{z F \Phi_{d}}{R T}, \quad X=\sqrt{8 c e R T}, \quad B=\frac{N_{1} z F}{1+\frac{N_{A} v}{M c}}
$$

Then Eq.(3.14) becomes

$$
p_{1}=\frac{B}{e^{-y_{2}}}=B e^{y_{1}}
$$

and Eq.(3.20) becomes

$$
p_{2}=X \sinh \left(\frac{y_{d}}{2}\right)
$$

Substitute (A2.2) and (A2.3) into Eq.(3.13)

$$
\begin{aligned}
p & =-\left(p_{1}+p_{2}\right) \\
& =-\left[B e^{y_{d}}+X \sinh \left(\frac{y_{d}}{2}\right)\right]
\end{aligned}
$$

Use the parameters listed from (1) to (10) to solve the Stern potential $\Phi_{d}$ :

(11) $\Phi_{\mathrm{d}}=0.133 \mathrm{~V}=139 \mathrm{mV}$; the Stern potential.

Sutstituice the relevant parameters from (1) to (10) into Eq.(3.15) anc olve 
for $\Phi_{0}$,

(12) $\Phi_{0}=3.17 \mathrm{~V}$; potential at particle surface.

(13) $\rho_{1}=0.231 \mathrm{C} / \mathrm{m}^{2}$; charge density in the Stern layer.

(14) $\rho_{2}=0.092 \mathrm{C} / \mathrm{m}^{2}$; charge density in the diffuse layer.

(15) the "thickness" of Gouy diffuse layer: $1 / x=3.04 \times 10^{-9} \mathrm{~m}$, Calculated from Eq.(3.19).

\section{Apparent Permittivity}

The apparent permittivity of the kaolinite system can be obtained by substitute the relaxant values into Eq.(3.34). Note that we assume the zeta potential is equivalent to the Stern potential in the calculation.

$$
\begin{aligned}
K_{E D L} & =7.65+ \\
& +\frac{9}{4} 0.44 \times 80\left[3 \times 0.1 \exp \left(\frac{96487 \times 0.139}{2 \times 8.314 \times 293}\right)+\exp \left(\frac{96487 \times 0.139}{2 \times 8.314 \times 293}\right)\right]^{2} \\
& =2.82 \times 10^{4}
\end{aligned}
$$




\section{APPENDIX 3 \\ ELIMINATION OF ELECTRODE POLARIZATION}

The effect of electrocie polarization may be modeled as a capacitance $C_{e}$ in parallel with the unknown capacitance of the measuring material $C_{x}$, as shown in Fig.A3.1. Thus the measured total capacitance $C_{m}$ is

$$
C_{m}=C_{x}+C_{e}
$$

The capacitance of a parallel capacitor can be computed as

$$
C=\frac{e A}{t}
$$

where $A$ is the electrode area, $t$ is the separation distance and $\epsilon$ is the permittivity of material between the electrode-plates.

It is known that the capacitance due to electrode polarization $\mathrm{C}_{\mathrm{e}}$ is caused by the local polarization at vicinity of the electrode plates, which is not affected by the separation distance of the electrode-plates. Thus by changing the separation distances between the electrode-plates, we save the equations

$$
\left\{\begin{array}{l}
c_{m l}=c_{x l}+c_{1} \\
c_{m 2}=c_{x 2}+c_{e}
\end{array}\right.
$$

where $C_{m 1}=$ measurt $\omega$ capacitance from $t=t_{1}=4 \mathrm{~cm}$;

$C_{m 2}=$ measured capacitance from $t=t_{2}=2 \mathrm{~cm}$;

$C_{x 1}=$ capacitance due to soil sample at $t=t_{1}=4 \mathrm{~cm}$;

$C_{x 2}=$ capacitance due to soil sample at $t=t_{2}=2 \mathrm{~cm}$. 
Eliminate $C_{e}$ from (A3.2)

$$
\begin{aligned}
C_{m 1}-C_{m 2} & =C_{x 1}-C_{x 2} \\
& =\operatorname{eA}\left(\frac{1}{t_{1}}-\frac{1}{t_{2}}\right)
\end{aligned}
$$

Solving for $\epsilon$

$$
e=\frac{c_{m 1}-C_{m}}{A\left(\frac{1}{t_{1}}-\frac{1}{t_{2}}\right)}
$$

Thus the permittivity of the material can be obtained by measuring $C_{m 1}$ and $C_{m 2}$ for different separation distances of electrode-plates.

\section{Example Calculation}

Consider the undisturbed Gloucester clay at in-situ water content of $63.8 \%$. Knowing the dimensions of the test cell

$$
\begin{aligned}
& t_{1}=0.04 \mathrm{~m} \\
& t_{2}=0.02 \mathrm{~m} \\
& A=0.1 \mathrm{~m} \times 0.1 \mathrm{~m}=0.01 \mathrm{~m}^{2}
\end{aligned}
$$

From the measurement we obtain

$$
\begin{aligned}
& C_{m 1}=4.01 \times 10^{-8} \mathrm{~F} / \mathrm{m} \\
& C_{m 2}=6.98 \times 10^{-8} \mathrm{~F} / \mathrm{m}
\end{aligned}
$$

Substitute these values into Eq.(A3.4), we obtain the relative permittivity of the undisturbed 
Gloucester clay

$$
\begin{aligned}
K & =\frac{\epsilon}{\epsilon_{\theta}} \\
& =\frac{(4.01-6.98) \times 10^{-8}}{0.01 \times\left(\frac{1}{0.04}-\frac{1}{0.02}\right) \times 8.85 \times 10^{-12}} \\
& =13.5 \times 10^{3}
\end{aligned}
$$

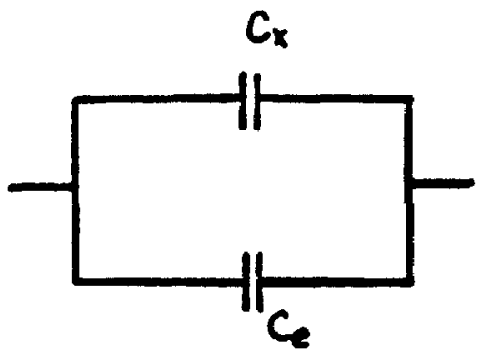




\section{APPENDIX 4 \\ X-RAY TRACES}

257 
258

BLOUCEOTER 2:RY

DEPT:

:FFU?

$\therefore$ ?. 0 .

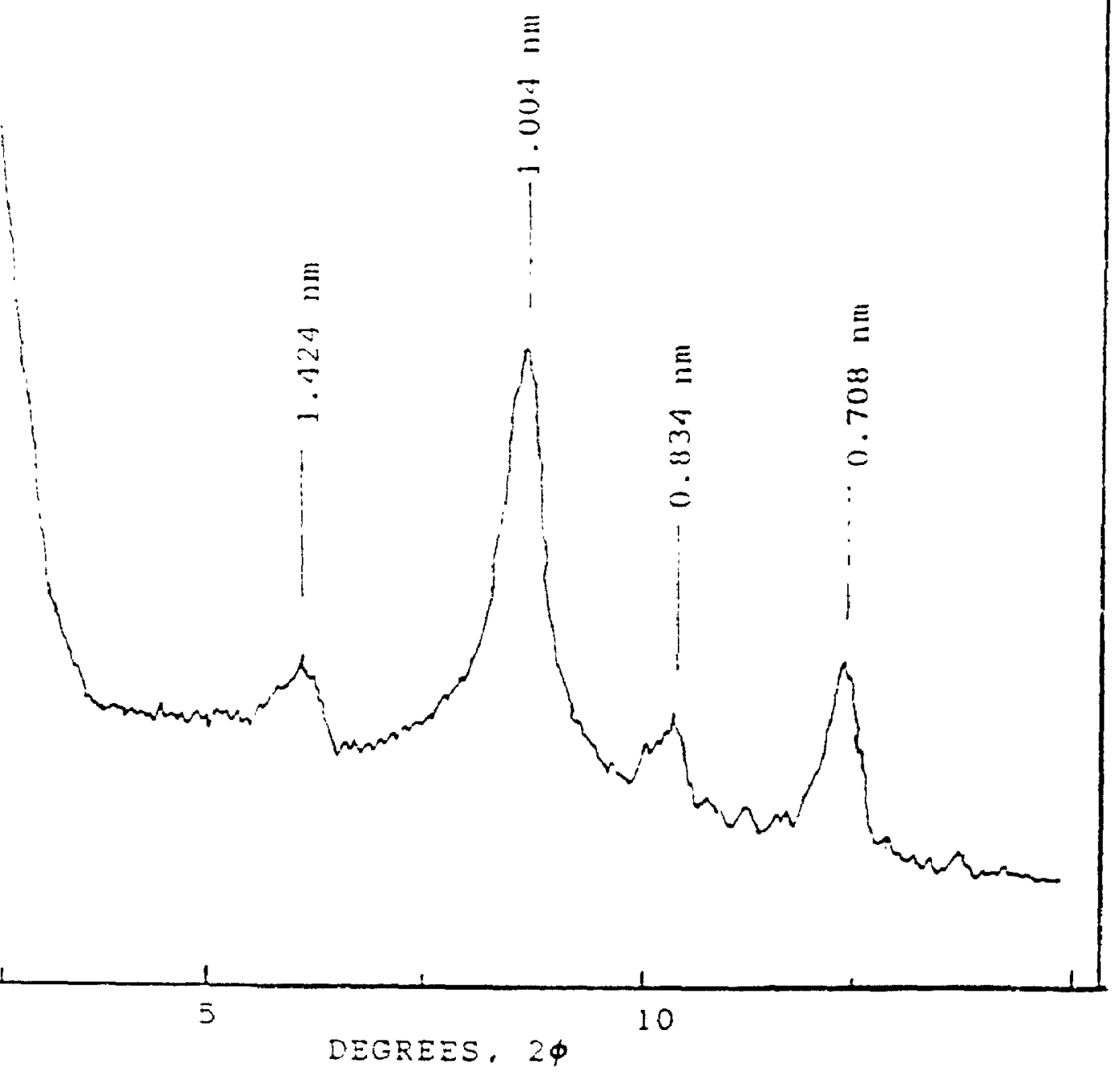

FIGURE A4.1 X-RAY DIFFRACTION TRACE OF GLOUCESTER CLAY 


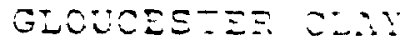

DEPT: $6 \mathrm{~m}$

AATLRAL

A. D.?.

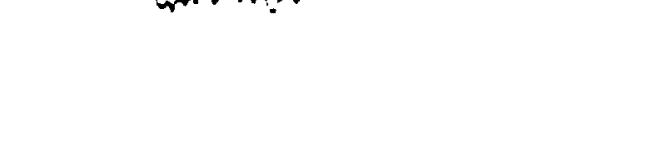




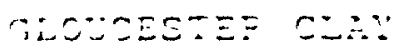

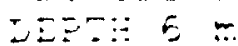

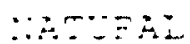

$\because .0$.

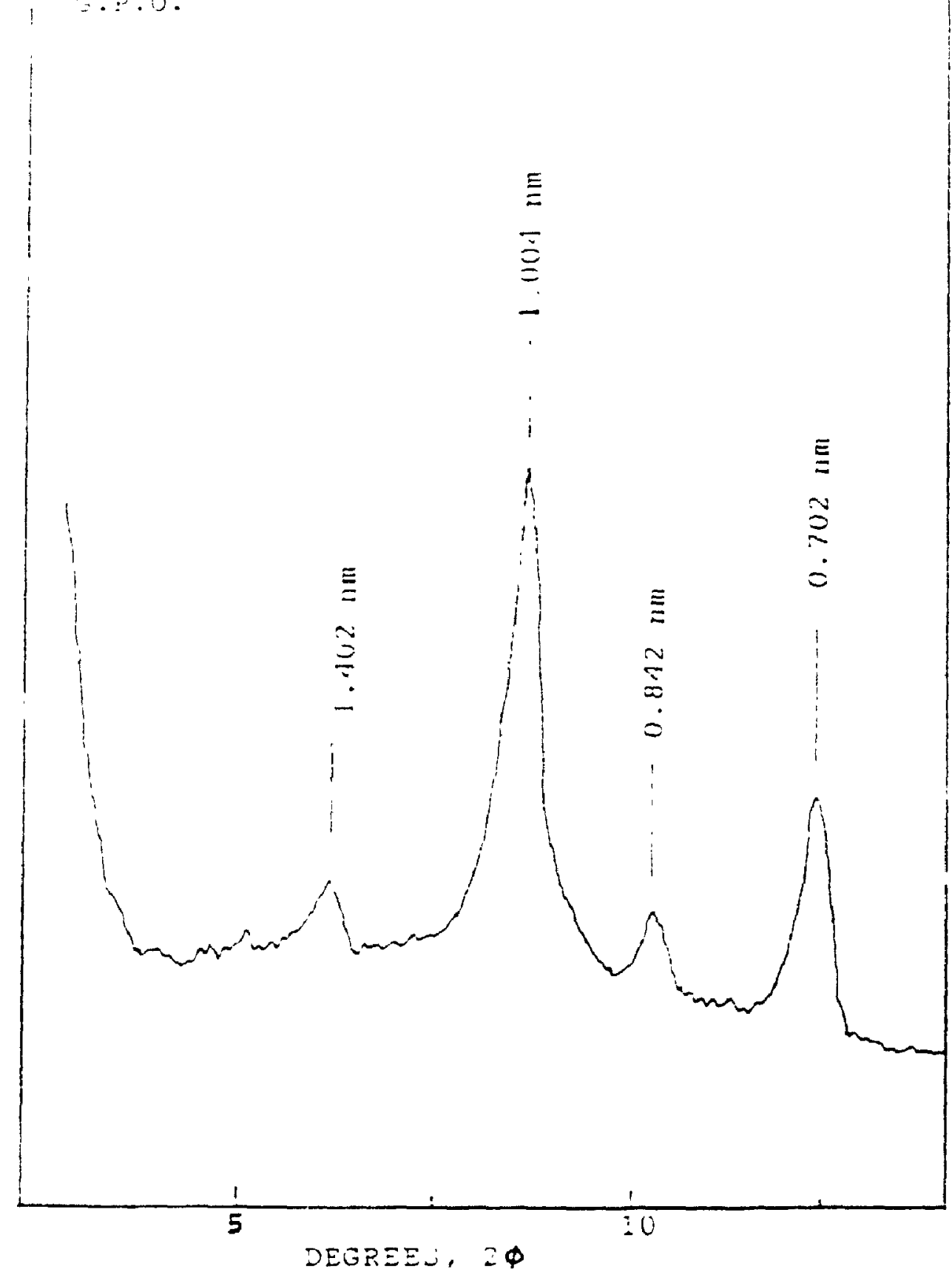

FIGURE A4.3 X-RAY DIFFRACTION TRACE OF GLOUCESTER CLAY 


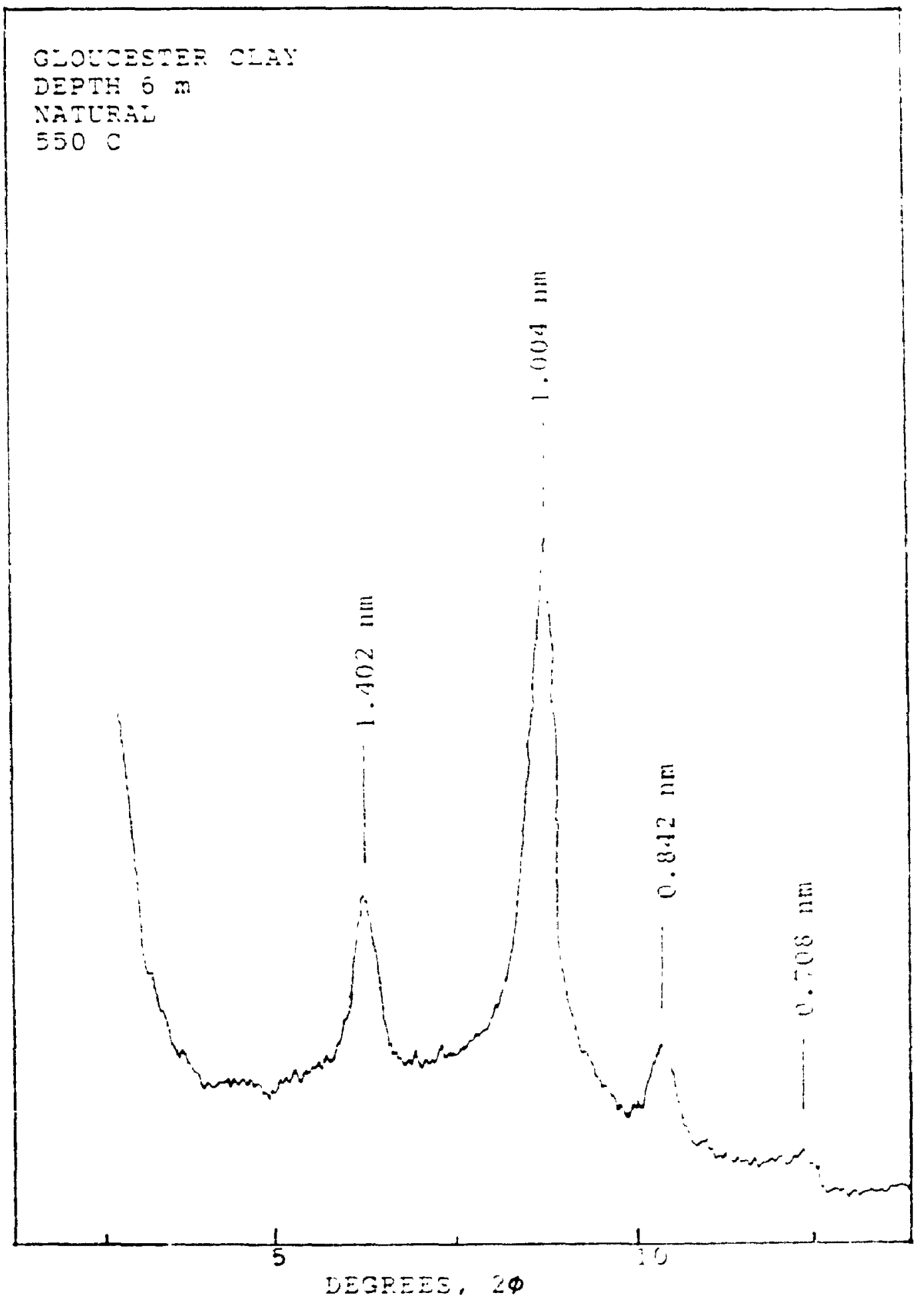

FIGURE A4.4 X-RAY DIFFRACTION TRACE OF GLOUCESTER CLAY 


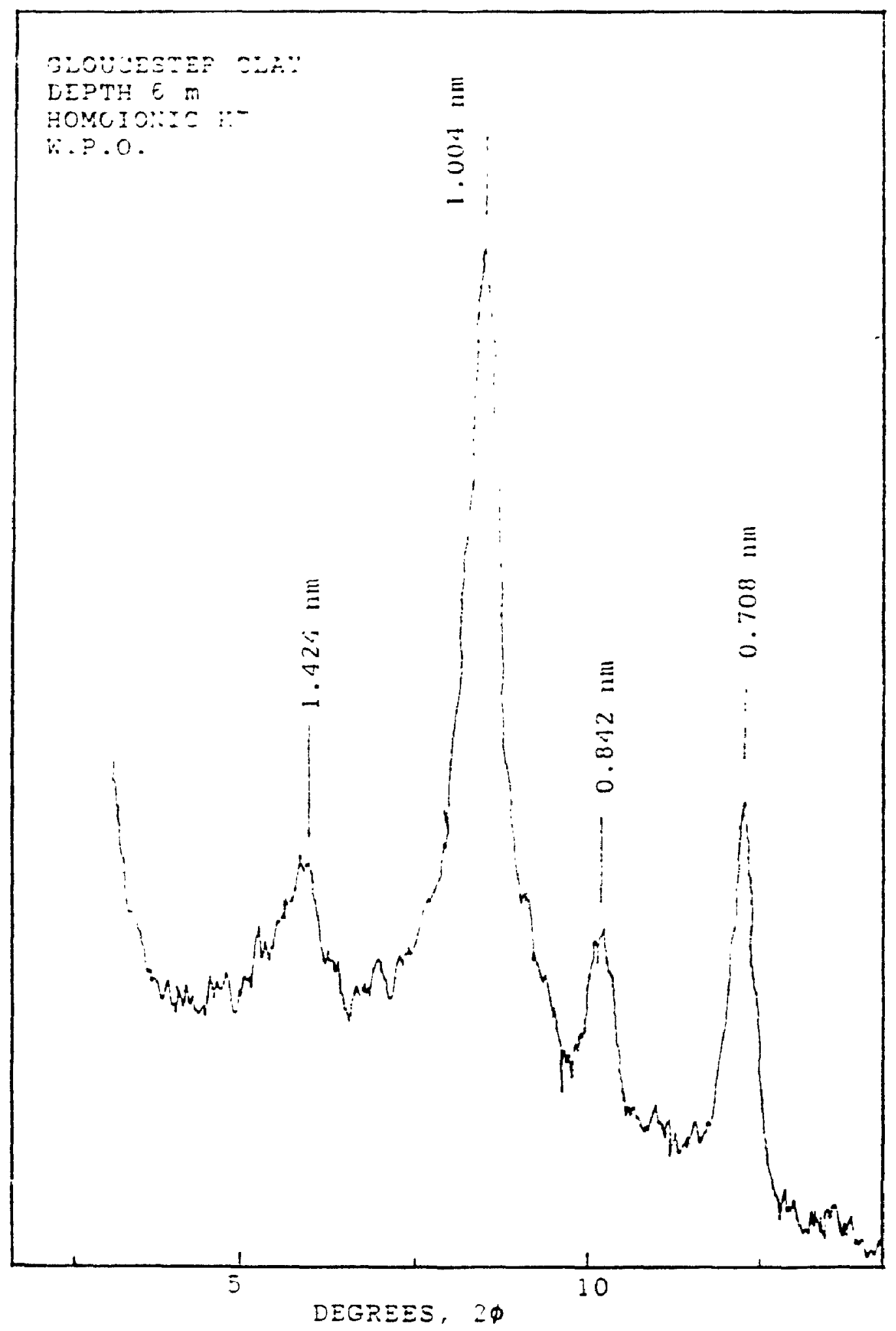

FIGURE A4.5 X-RAY DIFFRACTION TRACE OF GLOUCESTER CLAY 


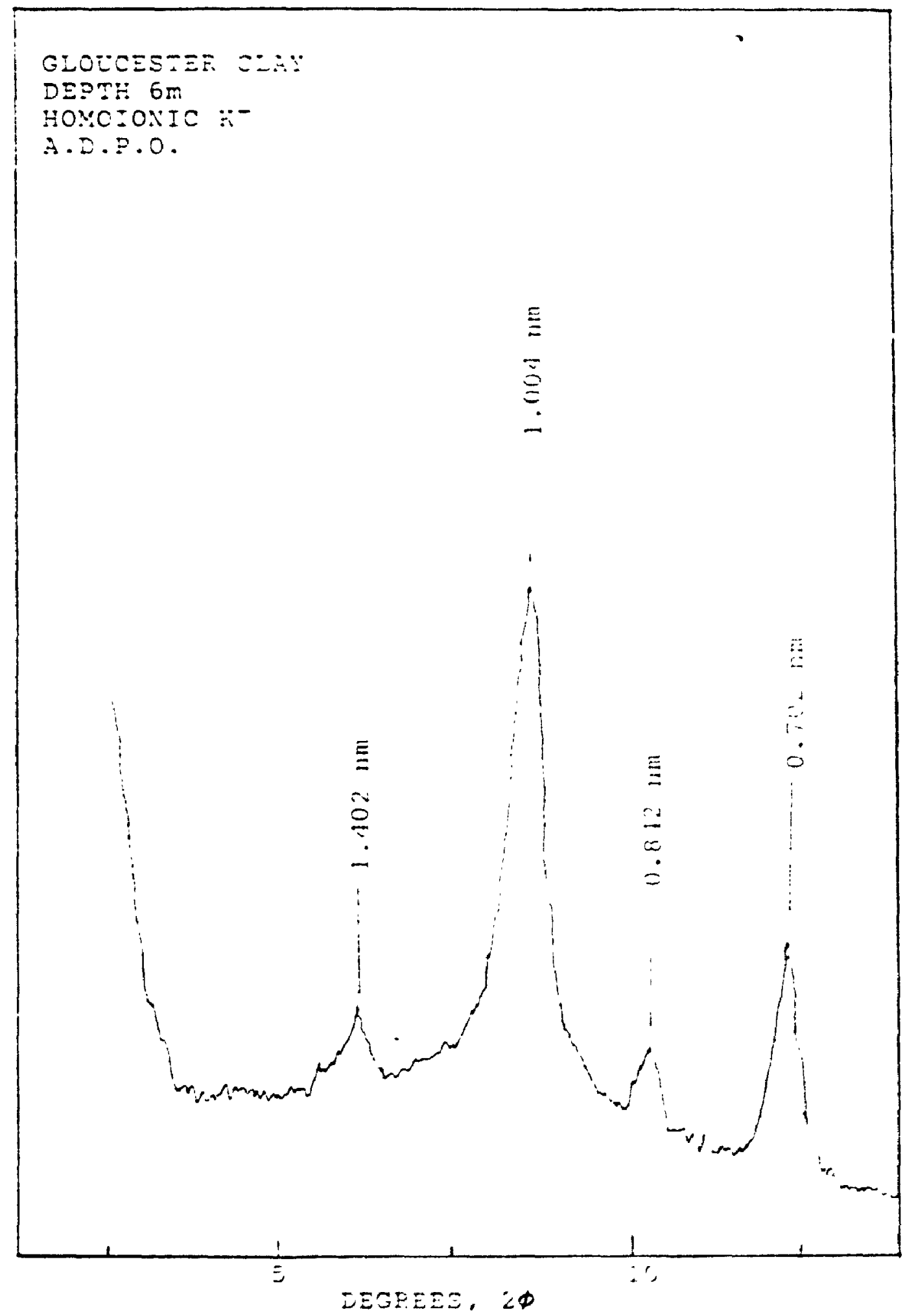

FIGURE A4.6 X-RAY DIFFRACTION TRACE OF GLOUCESTER CLAY 


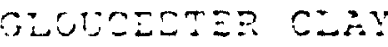

DEET:

iarouga:

$3 . \therefore$

\begin{tabular}{l}
$\equiv$ \\
$\Xi$ \\
\hline
\end{tabular}

$\Xi$
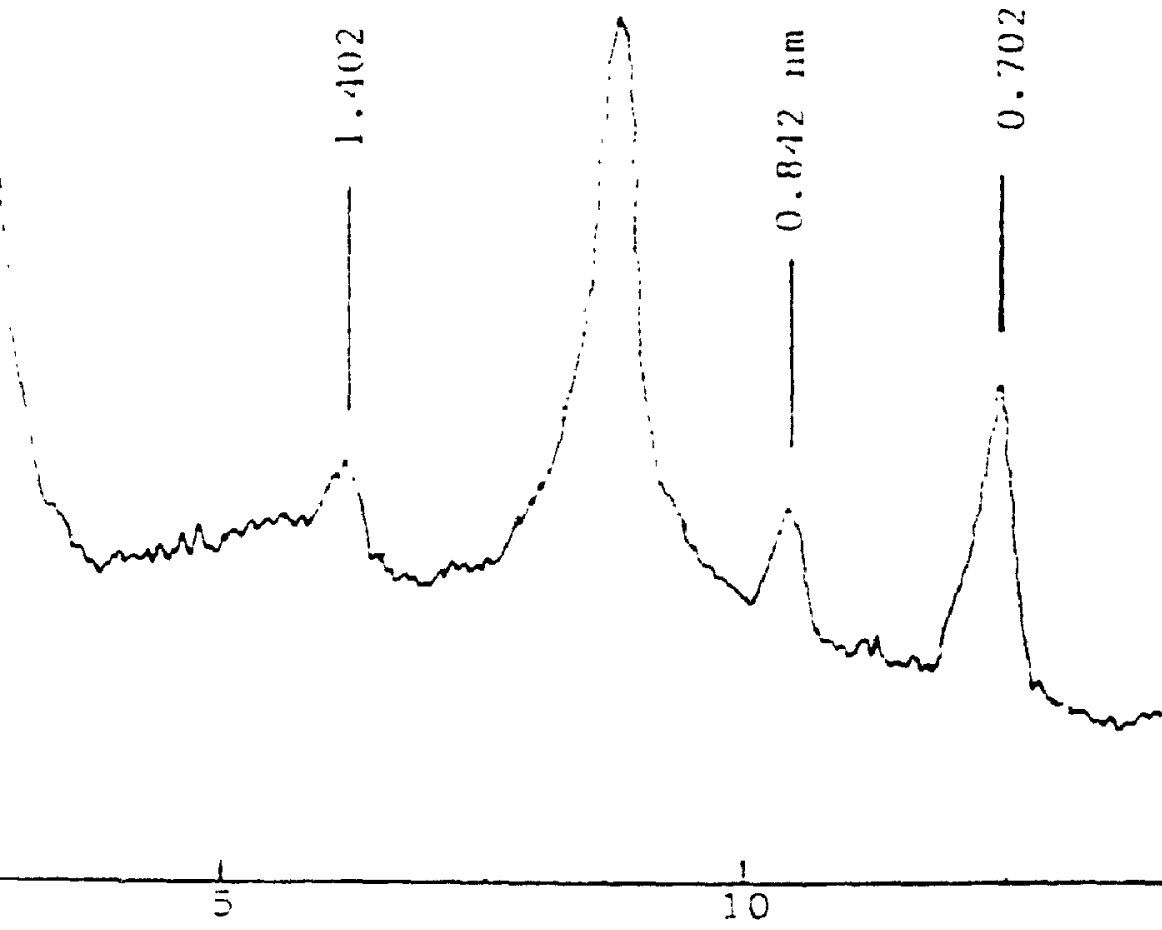

DEEREミS, 2Ф

FIGURE A4.7 X-RAY DIFFRACTION TRACE OF GLOUCESTER CLAY 


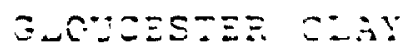

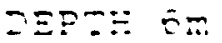

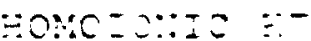

$\equiv \equiv 0 \Omega$

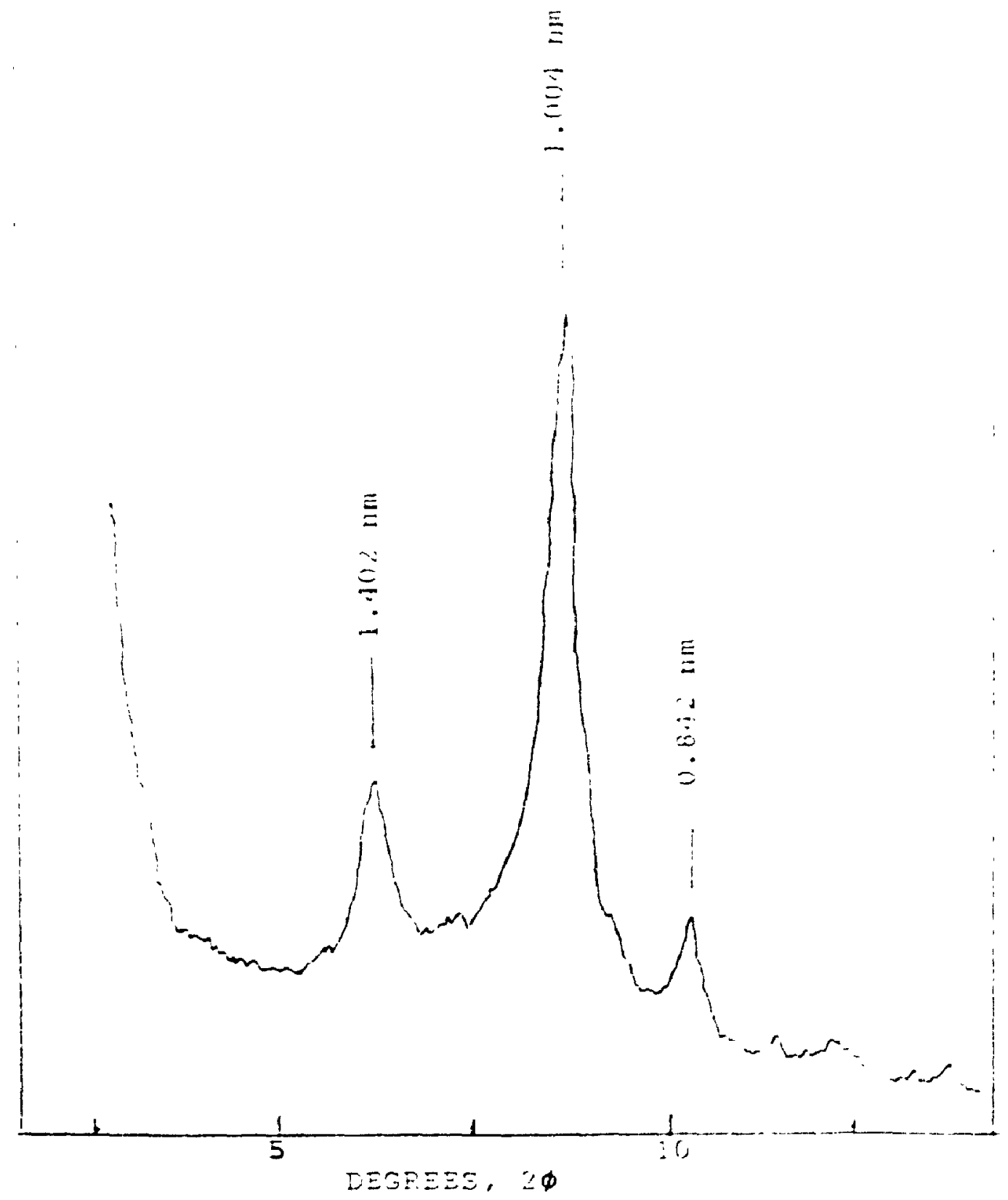

FIGURE A4.8 X-RAY DIFFRACTION TRACE OF GLOUCESTER CIAY 

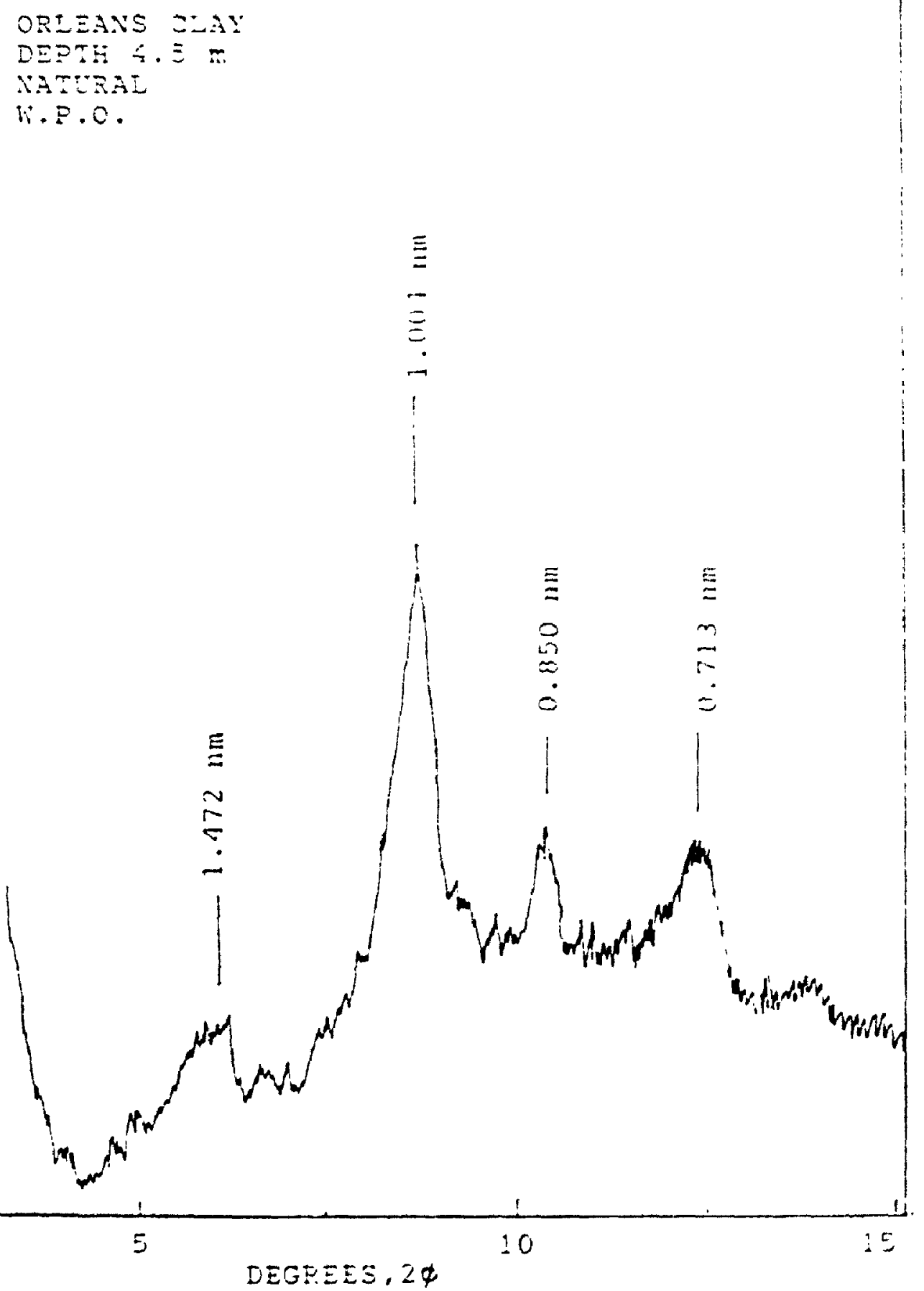

FIGURE A4.9 X-RAY DIFFRACTION TRACE OF ORLEANS CLAY 


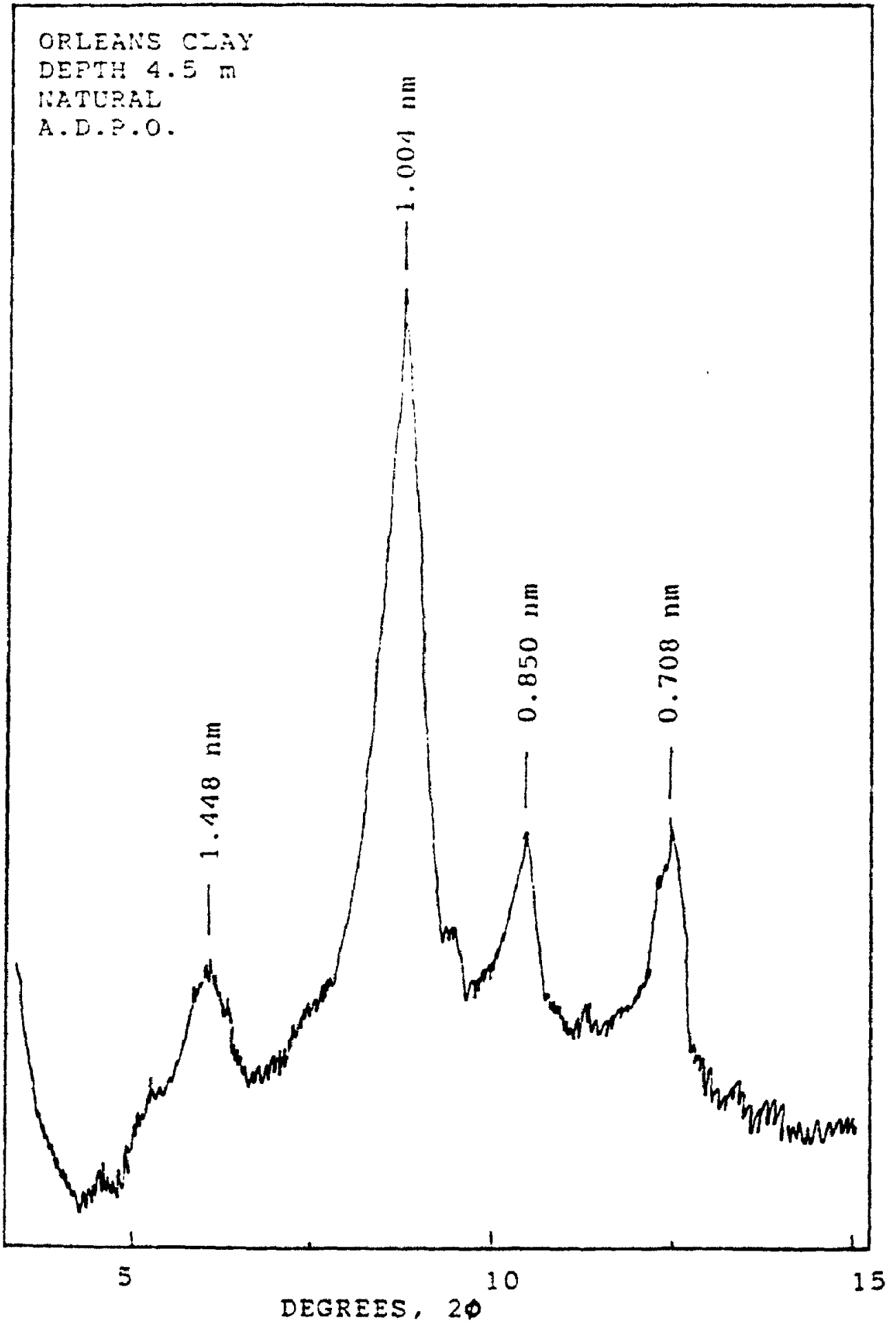

FIGURE A4.10 X-RAY DIFFRACTION TRACE OF ORIEANS CLAY 


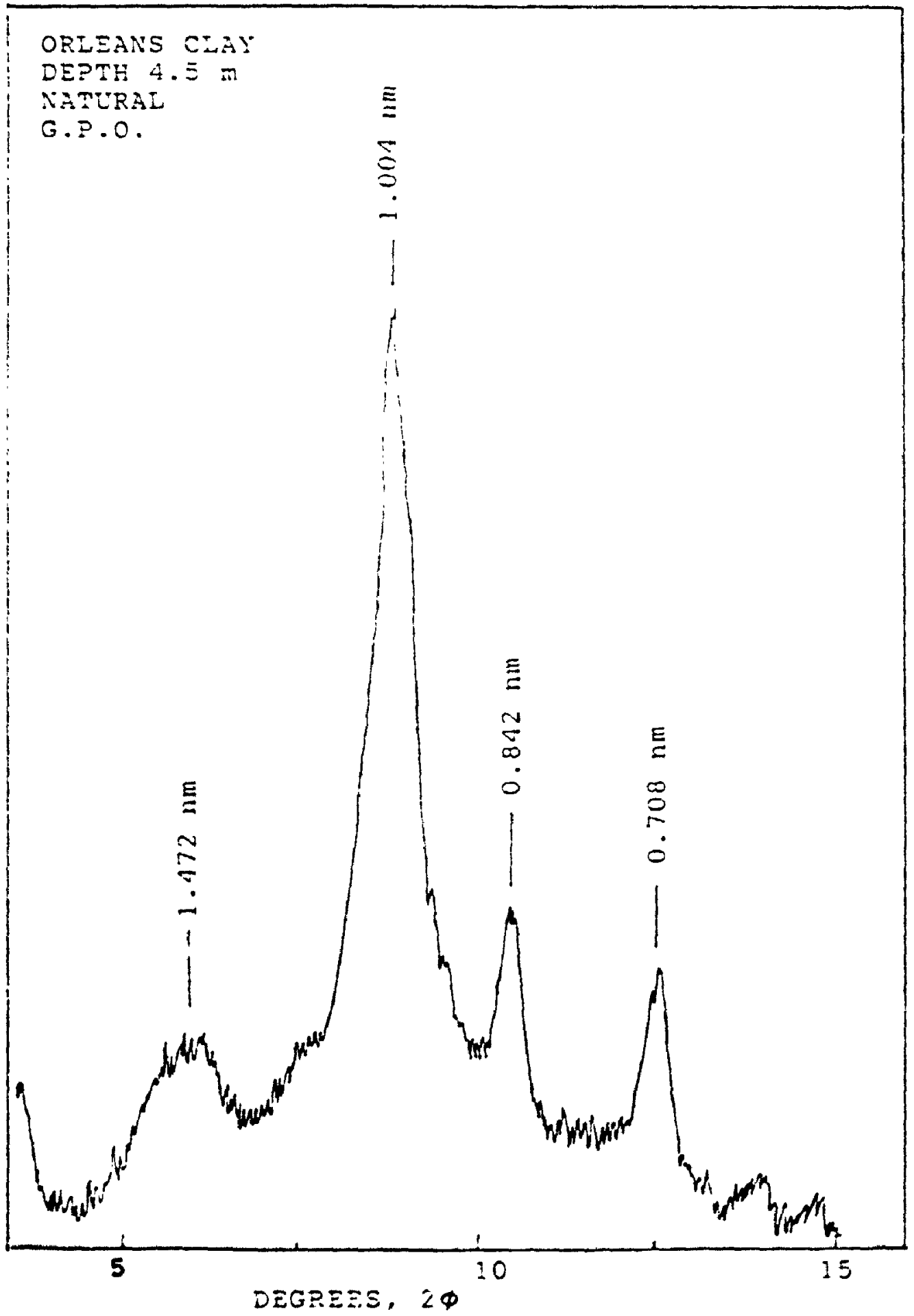

FIGURE A4.11 X-RAY DIFFRACIION TRACE OF ORIEANS CLAY 
ORIEALS CEAY

DEPTH $4 . \equiv \mathrm{m}$

NITURAL

$550 \mathrm{C}$

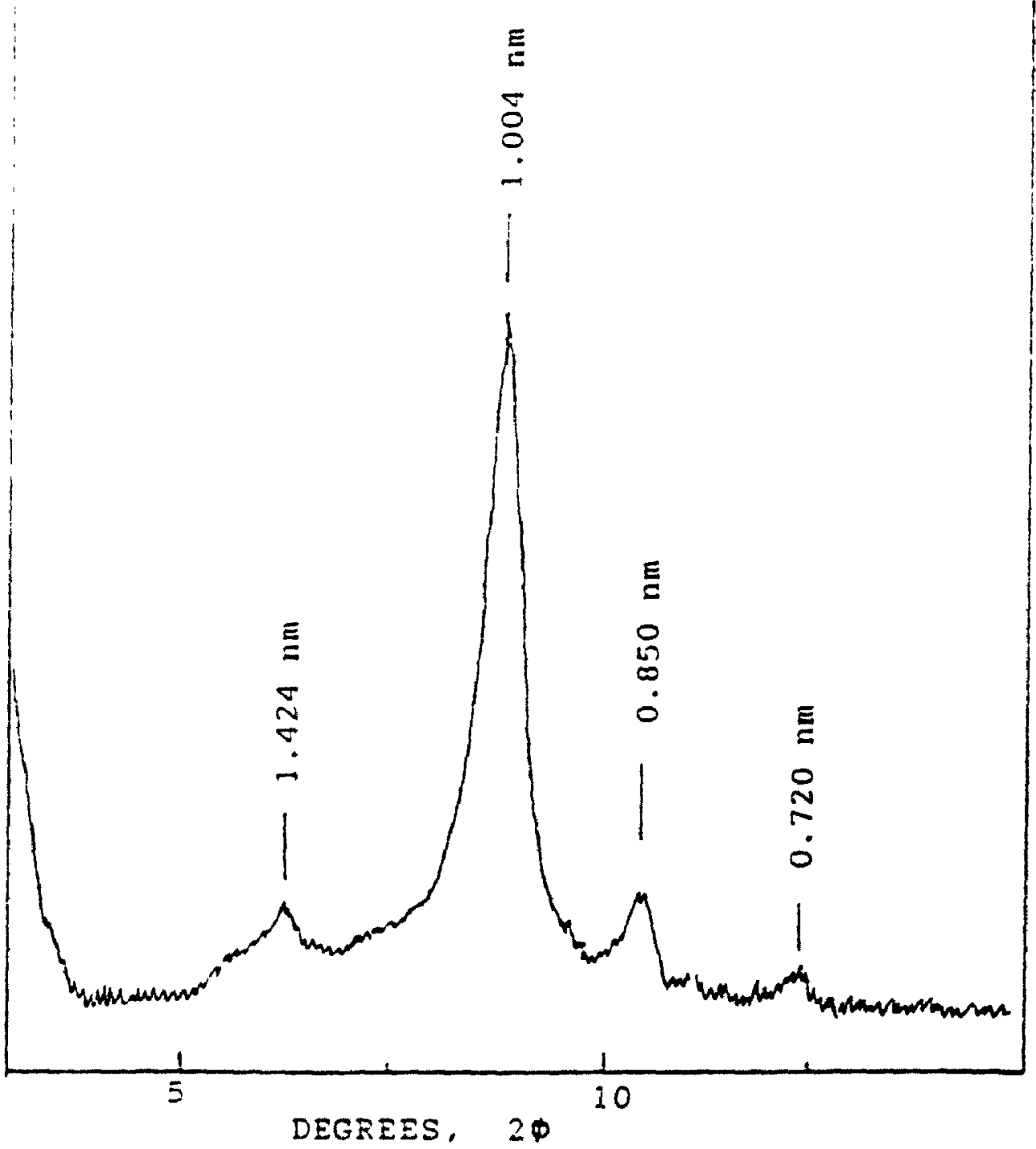

FIGURE A4.12 X-RAY DIFFRACTION TRACE OF ORLEANS CLAY 


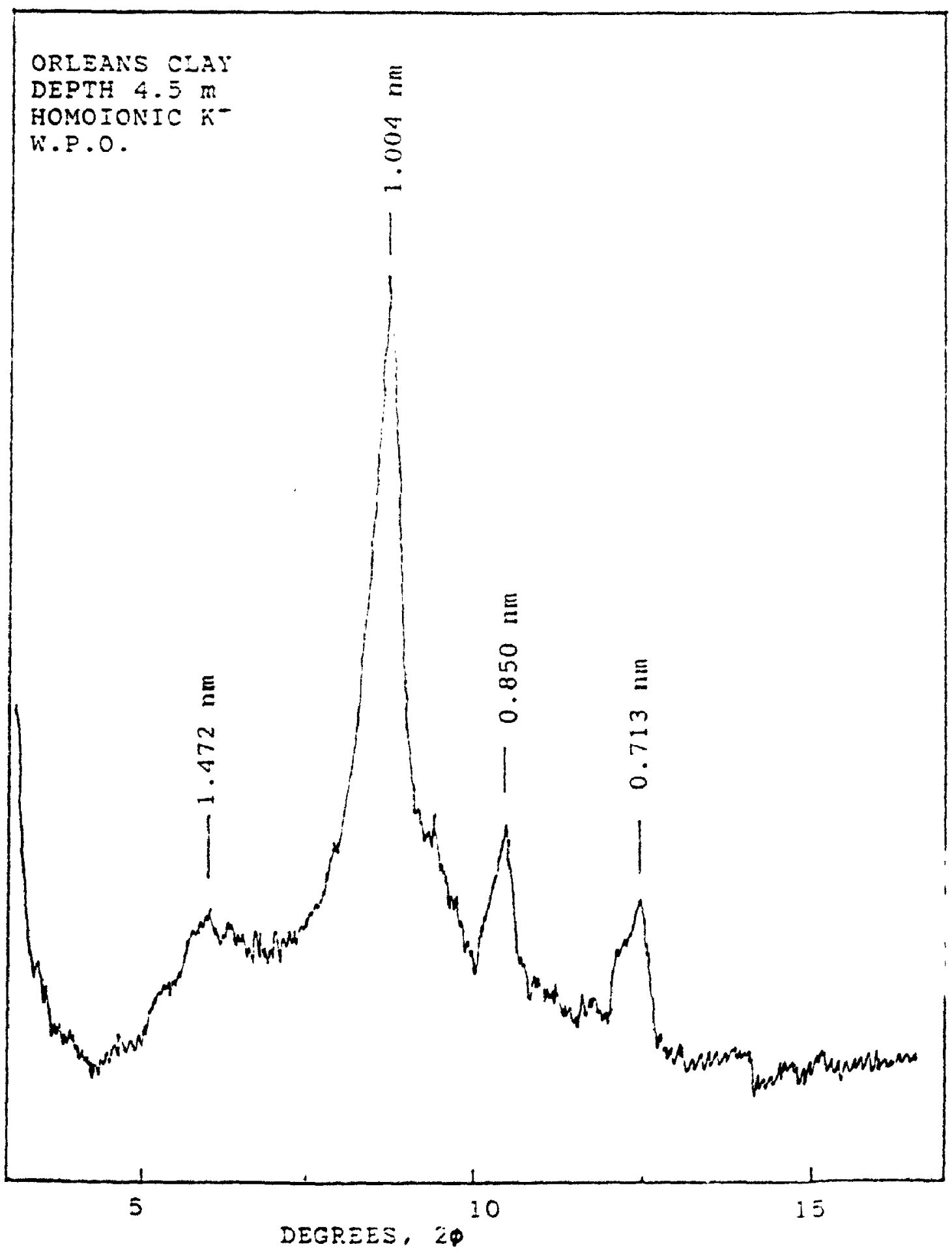




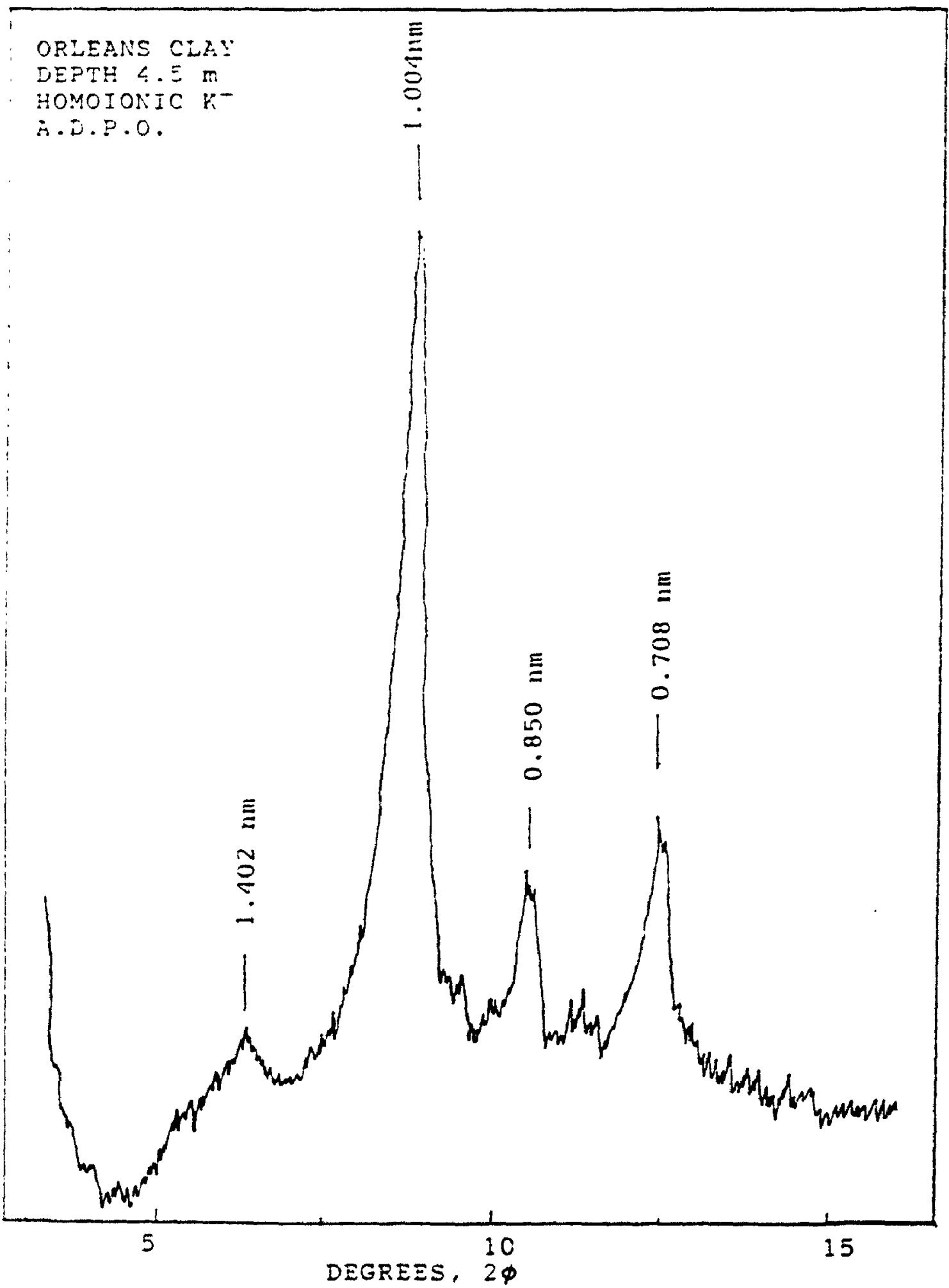

FIGURE A4.14 


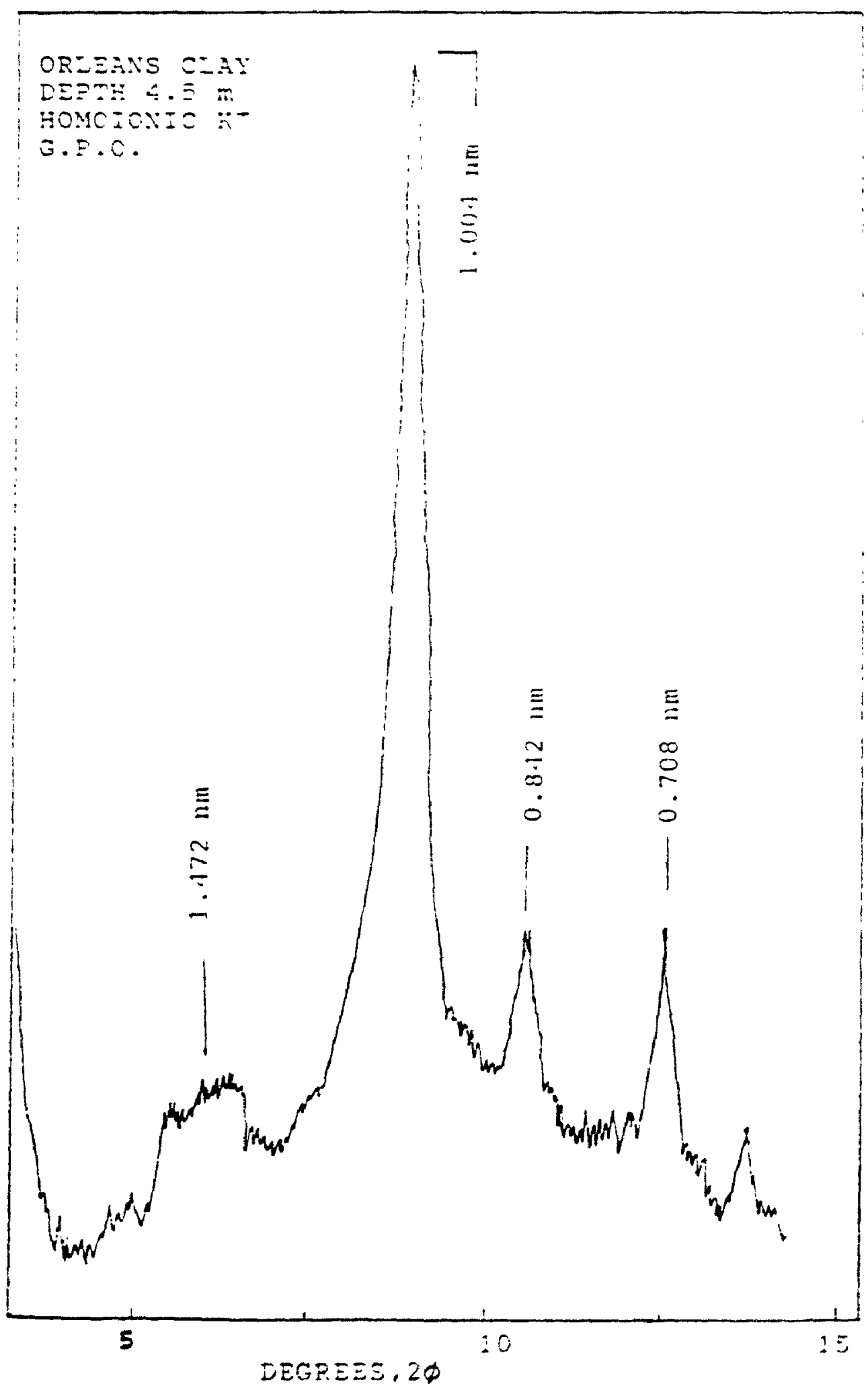

FIGURE A4.15 X-RAY DIFFRACTION TRACE OF ORLEANS CLAY 


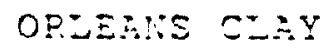

DEPT: $4.5 \pi$

nomeioni $=\mathrm{r}^{\top}$

$\equiv \equiv 0 \quad 0$

E

$\frac{5}{8}$

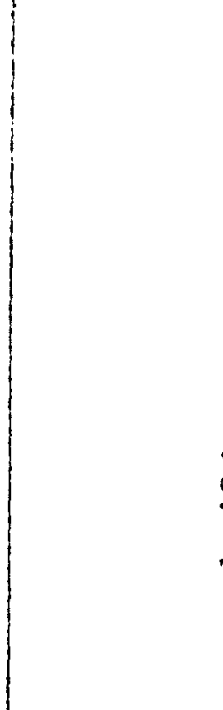
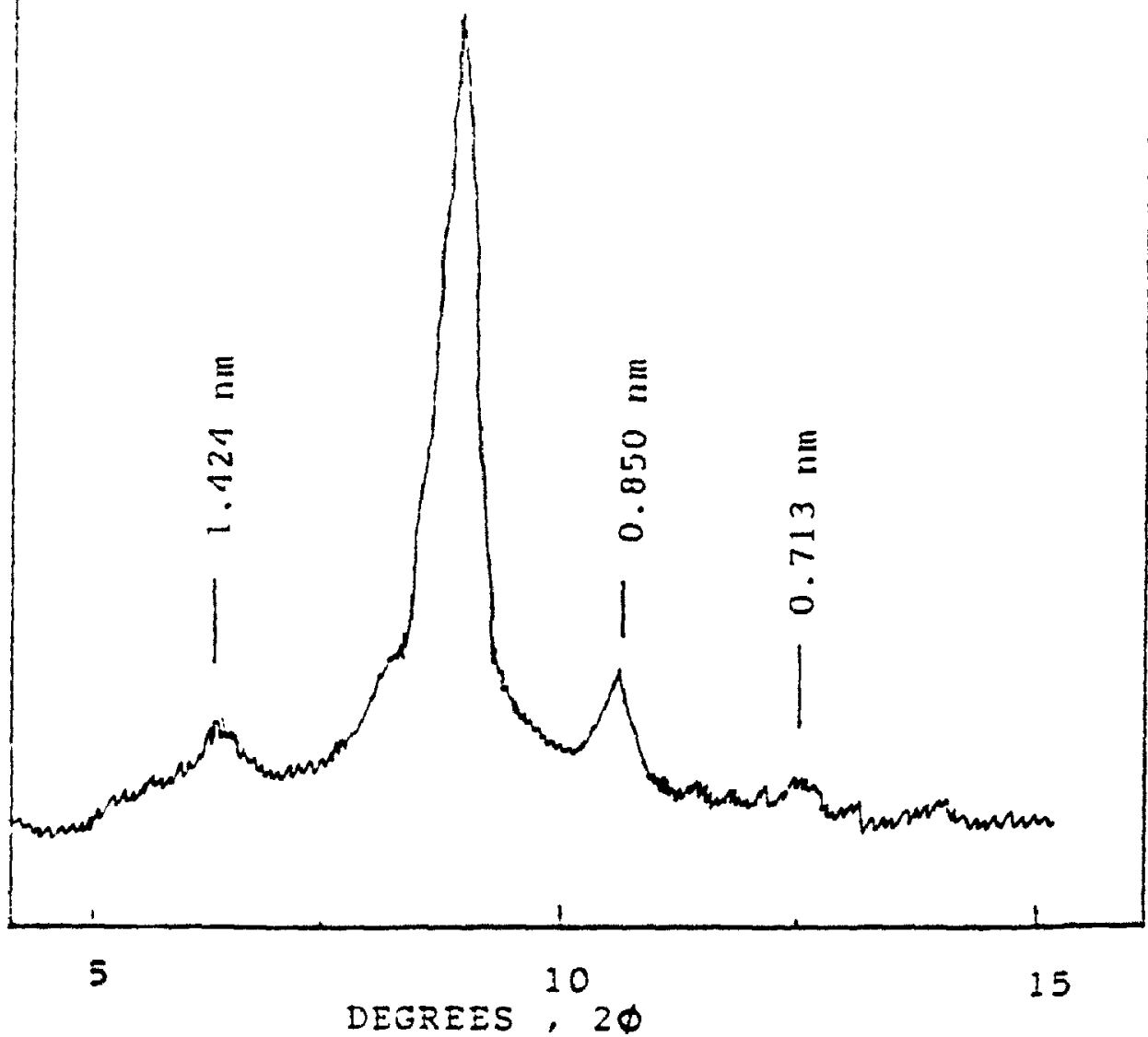

FIGURE A4.16 X-RAY DIFFRACTION TRACE OF ORIEANS CLAY 